\title{
An Improved Neutrino Oscillations Analysis of the MiniBooNE Data
}

\author{
Alexis Armando Aguilar-Arévalo
}

Submitted in partial fulfillment of the requirements for the degree of Doctor of Philosophy in the Graduate School of Arts and Sciences

COLUMBIA UNIVERSITY 2008 
(c) 2008

Alexis Armando Aguilar-Arévalo

All Rights Reserved 


\title{
ABSTRACT
}

\section{An Improved Neutrino Oscillations Analysis of the MiniBooNE Data}

\begin{abstract}
Alexis Armando Aguilar-Arévalo
We calculate the exclusion region in the parameter space of $\nu_{\mu} \rightarrow \nu_{e}$ oscillations of the LSND type using a combined fit to the reconstructed energy distributions of neutrino candidate samples from the MiniBooNE data obtained with two different particle identification methods. The two $\nu_{e}$ candidate samples are included together with a high statistics sample of $\nu_{\mu}$ events in the definition of a $\chi^{2}$ statistic which includes the correlations between the energy intervals of all three samples and handles the event overlap between the $\nu_{e}$ samples. The $\nu_{\mu}$ sample is introduced to constrain the effect of systematic uncertainties. This analysis increases the exclusion limit in the region $\Delta m^{2} \lesssim 1 \mathrm{eV}^{2}$ when compared with the result previously published by the collaboration, which used a different technique.
\end{abstract}




\section{Contents}

List of Tables $\quad$ V

List of Figures viii

Acknowledgments $\quad$ xiv

Introduction 1

1 Neutrinos $\quad 8$

1.1 Field Theory of massive fermions . . . . . . . . . . . . . . . 8

1.2 Neutrino mixing . . . . . . . . . . . . . . . . . . 14

1.3 Direct limits on the neutrino mass . . . . . . . . . . . . . . 18

1.4 Experimental observations of neutrino oscillations . . . . . . . . . 23

2 The MiniBooNE Experiment 35

2.1 The proton beam of the Booster Neutrino line . . . . . . . . . . 35

2.2 Target, Focusing Horn, and decay pipe . . . . . . . . . . . . 36

2.3 Neutrino beam and detector . . . . . . . . . . . . . . . 39 
2.4 Data Acquisition and Calibration systems . . . . . . . . . . . . . 42

2.5 Neutrino events in the tank . . . . . . . . . . . . . 54

$3 \quad$ Experiment Simulation and Reconstruction algorithms $\quad 57$

3.1 Beam simulation . . . . . . . . . . . . . . 57

3.2 NUANCE cross section generator ............... 74

3.3 Detector simulation . . . . . . . . . . . . . . . 88

3.4 Event Reconstruction algorithms . . . . . . . . . . . . . 97

3.5 Particle Identification Methods . . . . . . . . . . . . . . . . . 109

4 Oscillation analysis with two $\nu_{e}$ candidate samples $\quad 126$

4.1 Analysis overview . . . . . . . . . . . . . . 126

4.2 The combined $\nu_{\mu} / \nu_{e}$ fit technique used in the BDT analysis. . . . . . 131

4.3 Oscillations results with the BDT analysis . . . . . . . . . . 146

4.4 Oscillations results with the TBL analysis . . . . . . . . . . 153

4.5 Combining the $\nu_{e} \mathrm{BDT}, \nu_{e} \mathrm{TBA}$, and $\nu_{\mu}$ CCQE samples $\ldots . . . . \quad 159$

$5 \quad$ Results and Conclusions $\quad 165$

5.1 Results . . . . . . . . . . . . . . . . . 165

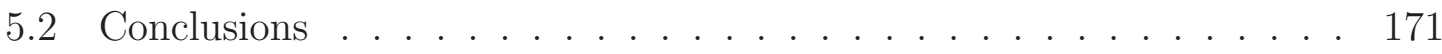

$\begin{array}{ll}\text { Bibliography } & 174\end{array}$

$\begin{array}{ll}\text { Appendices } & 190\end{array}$ 
A.1 Selecting well reconstructed QE events . . . . . . . . . . . . . . . 191

A.2 Calibration Procedure . . . . . . . . . . . . . . . . . . 191

$\begin{array}{lll}\text { B Understanding the } \nu_{\mu} \text { constraint } & 199\end{array}$

B.1 Example: $1-\nu_{e}$ bin $+1-\nu_{\mu}$ bin . . . . . . . . . . . . . . . 199

C A frequentist study with the BDT analysis. 208

C.1 Introduction . . . . . . . . . . . . . . . . . 208

C.2 Finding the best fit oscillation parameters . . . . . . . . . . . . . 209

C.3 Ordering Principle . . . . . . . . . . . . . . . . . . . . . 210

C.4 Alternative possibilities . . . . . . . . . . . . . . . 212

C.5 Example with a $6 \times 6$ grid . . . . . . . . . . . . . . 213

C.6 Generalized Frequentist Sensitivity . . . . . . . . . . . . . . . 220

D Frequentist $3 \sigma$ and $5 \sigma$ C.L. contours with limited numbers of fake $\begin{array}{ll}\text { experiments. } & 228\end{array}$

D.1 Introduction . . . . . . . . . . . . . . . . . . . 228

D.2 The Gamma Distribution and the Incomplete Gamma Function . . . 229

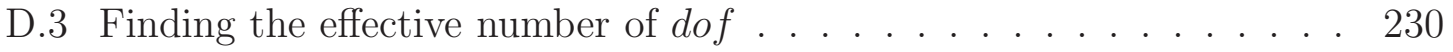

D.4 Conclusions . . . . . . . . . . . . . . . . . . . . . . 238

E Frequentist contours for the MiniBooNE BDT analysis. 239

E.1 Introduction . . . . . . . . . . . . . . . . . . . . . . . . 239 
E.2 Results . . . . . . . . . . . . . . . . . . . . . . . . . . 240

E.3 Effective number of $d o f \ldots \ldots \ldots \ldots \ldots$

F Miscellaneous tests of the fits 245

F.1 Tests of statistical correlations with systematic errors . . . . . . . 245

F.2 Slices of the $\chi^{2}$ surface . . . . . . . . . . . . . . . . 251

F.3 Example fits to fake data sets . . . . . . . . . . . . . . 251

G Events from the NuMI Beamline $\quad 261$

G.1 The NuMI Trigger in MiniBooNE . . . . . . . . . . . . . . . . 261

H Geometry of the NuMI and MiniBooNE Beamlines 277

H.1 Positions of elements in site coordinates . . . . . . . . . . 277

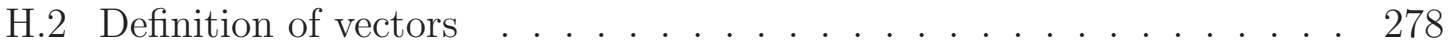

H.3 Aereal view projections of the beamlines . . . . . . . . . . 279

H.4 MiniBooNE position in NuMI coordinates . . . . . . . . . 280

H.5 Basis transformation Matrices . . . . . . . . . . . . . . 280

H.6 Production line vectors in MB coordinates . . . . . . . . . . . 282

H.7 Rotation matrices in polar angle format . . . . . . . . . . . 282

H.8 Transformation matrix from NuMI to MB: . . . . . . . . . . . . 283 


\section{List of Tables}

1.1 Left handed isospin doublets and right handed isospin singlets in the Standard Model. . . . . . . . . . . . . . . . . . . . 9

1.2 Direct limits on neutrino masses. . . . . . . . . . . . . . . . . 22

1.3 Short baseline neutino oscillation searches. . . . . . . . . . . . . . 31

2.1 Laser flask positions. . . . . . . . . . . . . . . . 46

2.2 Internal trigger bits and their thresholds. . . . . . . . . . . . . 52

3.1 Threshold production channels for $p+p$ production of mesons. . . . . 58

3.2 Covariance matrix, Sanford-Wang $\pi^{+}$production parameterization. . 62

3.3 Covariance matrix, Sanford-Wang $\pi^{-}$production parameterization. . 63

3.4 Covariance matrix, Feynman Scaling $K^{+}$production parameterization. 65

3.5 Covariance matrix, Sanford-Wang $K^{0}$ production parameterization. . 67

3.6 MiniBooNE flux predictions per neutrino type. . . . . . . . . . . 69

3.7 Systematic uncertainties from beam modeling. . . . . . . . . . . 72

3.8 Covariance matrix, $\mathrm{NC} \pi^{0}$ yield correction in momentum bins. . . . . 87

3.9 NUANCE cross section parameters with uncertainties. . . . . . . 88 
3.10 Optical model parameters. . . . . . . . . . . . . . . . . . . 95

3.11 Boxes used in the PID input validation of the BDT algorithm. . . . 114

4.1 Expected events passing the $\nu_{e}$ candidate selection cuts. . . . . . . 130

4.2 Systematic uncertainties for $100 \%$ full $\nu_{\mu} \rightarrow \nu_{e}$, BDT events. . . . . 140

4.3 Systematic uncertainties, BDT backgrounds. . . . . . . . . . . . . 141

4.4 Systematic uncertainties, $\nu_{\mu}$ CCQE events. . . . . . . . . . 143

4.5 Systematic uncertainties for $100 \%$ full $\nu_{\mu} \rightarrow \nu_{e}$, TBL events. . . . . 151

4.6 Systematic uncertainties, TBL backgrounds. . . . . . . . . . . . 152

5.1 Counting experiment results. . . . . . . . . . . . . . 167

C.1 $\chi^{2}$ values for central confidence intervals. . . . . . . . . . . 214

D.1 $\chi^{2}$ values for central confidence intervals. . . . . . . . . . . 231

D.2 Comparison of $d o f_{\text {eff }}$ obtained with Methods I and II. . . . . . . . 233

G.1 Relevant times for the NuMI trigger settings. . . . . . . . . . . . 264

G.2 Quality cuts applied to select good spills from the NuMI beam. . . . 267

G.3 Selection cuts for a sample of exiting NuMI events. . . . . . . . . . 269

G.4 Measurements of the NuMI production line geometry. . . . . . . . . 269

G.5 Flux from the NuMI beamline at MiniBooNE per neutrino type. . . . 274

H.1 Location of NuMI and MiniBooNE beamline elements. . . . . . . . 278

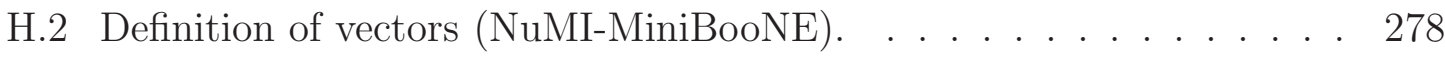

H.3 Magnitude of vectors defining the NuMI and MiniBooNE baemlines. . 280 
H.4 Polar angles of NuMI and MiniBooNE coordinate axes in SITE coordinates. . . . . . . . . . . . . . . . . . 283

H.5 Polar angles of MiniBooNE coordinate axes in NuMI coordinates. . . 284 


\section{List of Figures}

1 Thesis Results I. Energy distributions. . . . . . . . . . . . . . . 4

2 Thesis Results II. Sensitivities and limits. . . . . . . . . . . . 5

3 Thesis results III. Comparing to first MiniBooNE results. . . . . . . . 6

1.1 Neutrino oscillations, toy example. . . . . . . . . . . . . . . 19

1.2 Tritium $\beta$ decay spectrum. . . . . . . . . . . . . . . . . . . 21

1.3 Established observations of neutrino oscillations. . . . . . . . . . 30

1.4 Excess events in LSND. . . . . . . . . . . . . . . . . 33

1.5 LSND allowed oscillation regions. . . . . . . . . . . . . . 34

2.1 The $8 \mathrm{GeV}$ beamline with extraction facility. . . . . . . . 37

2.2 The MiniBoonE Be target. . . . . . . . . . . . . . 38

2.3 MiniBooNE detector schematic and optical barrier. . . . . . . . . . 40

2.4 Detector containment plant. . . . . . . . . . . . . . . . . . . . 41

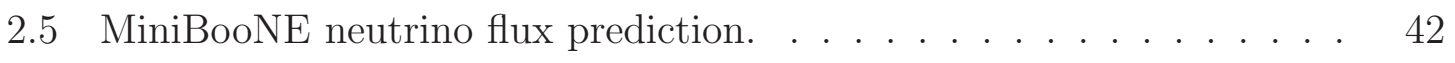

2.6 PMT charge and time signals. . . . . . . . . . . . . . . . . . 43

2.7 Corrected time distributions of laser events. . . . . . . . . . . 47 
2.8 MiniBooNE calibration systems. . . . . . . . . . . . . . . . . . . . 48

2.9 Muon tracker and cubes energy calibration. . . . . . . . . . 50

2.10 Michel electron energy spectrum. . . . . . . . . . . . . . . . 51

2.11 Example PMT hit times for one and two sub-event events. . . . . . 54

2.12 Neutrino interactions in the tank. The $N_{\text {tank }}$ and $N_{\text {veto }}$ cuts. . . . 55

3.1 Summary of $p B e \rightarrow \pi^{+} X$ experiments for $\pi^{+}$parameterization. . . . 61

3.2 Summary of $p B e \rightarrow K^{+} X$ experiments for $K^{+}$parameterization. . . 64

3.3 Summary of $p B e \rightarrow K^{0} X$ experiments for $K^{0}$ parameterization. . . . 66

3.4 MiniBooNE flux prediction per neutrino type. . . . . . . . . 68

3.5 Cross-sections for $p / n$ and $\pi^{+}$interactions in Beryllium. . . . . . 73

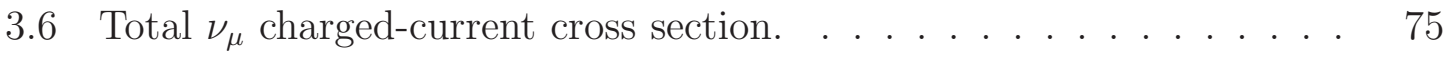

3.7 Feynman diagram for the CCQE process on a free nucleon. . . . . . 76

3.8 NUANCE prediction for the $\nu_{\mu}$-CCQE cross section. . . . . . . . 81

3.9 CCQE neutrino scattering on a neutron at rest. . . . . . . . . 82

3.10 Neutral Current resonant $\pi^{0}$ production. . . . . . . . . . . 83

3.11 Reconstructed momentum distribution of $\pi^{0}$ events. . . . . . . . 85

3.12 Reconstructed $\pi^{0}$ mass in reconstructed $\pi^{0}$ momentum bins. . . . . 86

3.13 GEANT3 rendering of the detector and surroundings. . . . . . . . 89

3.14 Wavelength dependence of photon attenuation length in oil. . . . . 92

3.15 Events from external interactions. . . . . . . . . . . . . . . . 94

3.16 Schematic of hit topologies for electrons, muons, and $\pi^{0}$ s. $\ldots \ldots .109$ 
3.17 The TBL $\nu_{e}$ CCQE selection cuts. . . . . . . . . . . . . . 110

3.18 Schematic of $\cos \theta$ and ring sharpness bins for BDT inputs. . . . . 113

3.19 Schematic of a decision tree. . . . . . . . . . . . . . . 116

3.20 AdaBoost progression of the misclassified event rate. . . . . . . . 117

$3.21 \nu_{e}$ efficiency of the TBL selection cuts. . . . . . . . . . . . . 121

3.22 The energy-dependent BDT score cut. . . . . . . . . . . . . 122

$3.23 \nu_{e}$ efficiency of the BDT selection cuts. . . . . . . . . . . 123

4.1 Reconstructed neutrino energy: $\nu_{\mu}$ CCQE, and $\nu_{e}$ CCQE samples. . . 129

4.2 Illustration of the BDT extended error matrix and its collapse. . . . 136

4.3 Reconstructed neutrino energy: $\nu_{e}$ BDT, $\nu_{e}$ TBL and $\nu_{\mu}$ CCQE. $\ldots . .147$

4.4 Sensitivity and limits to oscillations, BDT analysis. . . . . . . . 148

4.5 Energy distributions, TBL analysis. . . . . . . . . . . . . 154

4.6 Sensitivity and limits to oscillations, TBL analysis. . . . . . . . 155

4.7 Sensitivities and limits to oscillations, TBL analysis (smoothed O.M.). 158

4.8 Illustration of the BDT+TBL extended error matrix and its collapse. 160

4.9 Overlapping and non-overlapping events, BDT and TBL data samples. 161

4.10 Testing statistical correlations. Fake data fit, stat. errors only (BDT). 163

4.11 Testing statistical correlations. Fake data fit, stat. errors only (TBL). 164

5.1 Sensitivities and limits. Final Results. . . . . . . . . . . . 168

5.2 Energy distributions. Final results. . . . . . . . . . . . . . . . . . 169

5.3 Comparing the results with published MiniBooNE results. . . . . . 170 
A.1 Reconstructed vs. true kinetic energy. . . . . . . . . . . . . . . . . . . 194

A.2 Reconstruction of $\nu_{\mu}$ CCQE kinematics. . . . . . . . . . . . . . 195

A.3 Reconstruction of $\nu_{e}$ CCQE kinematics. . . . . . . . . . . . 196

A.4 Distribution of $\Delta_{E_{\nu}}$ reconstructed $E_{\nu}^{Q E}-$ generated $E_{\nu}^{\text {true }} \ldots \ldots$. . . . 197

A.5 Neutrino energy resolution for CCQE events. . . . . . . . . . . . . 198

C.1 Region ordering principle. . . . . . . . . . . . . . . . . . . . . . . 210

C.2 $\Delta \chi_{c}^{2}$ distributions for two points in the grid. . . . . . . . . . . . . 215

C.3 $\Delta \chi^{2}$ distributions, $6 \times 6$ grid. . . . . . . . . . . . . . 216

C.4 $\Delta \chi_{c}^{2}(90 \%)$ surface. . . . . . . . . . . . . . . . . . . 217

C.5 Stat+Systematic errors: $\Delta \chi^{2}$ distributions, $6 \times 6$ grid. . . . . . . . 218

C.6 Stat+Systematic errors: Surface of the $\Delta \chi_{c}^{2}(90 \%)$ cut values. . . . . . 219

C.7 Statistical errors: $\Delta \chi_{\text {null }}^{2}$ distributions, $6 \times 6$ grid. . . . . . . . . . 223

C.8 Statistical errors: Schwetz method sensitivity. . . . . . . . . . . . . . 224

C.9 Stat+Systematic errors: $\Delta \chi_{\text {null }}^{2}$ distributions, $6 \times 6$ grid. . . . . . . 225

C.10 Stat+Systematic errors: Schwetz method sensitivity. . . . . . . . . . . 226

C.11 90\% C.L. contours from the two methods described. . . . . . . . . . . 227

D.1 Implementation of the change of variable to yield the CDF $F(x) \ldots$. . 230

D.2 $\Delta \chi^{2}$ distributions for six points in the grid. . . . . . . . . . 235

D.3 Effective number of $d o f$ from Method I and II. . . . . . . . . . . . . . 236

D.4 Feldman-Cousins contours. Study case. . . . . . . . . . . . . . 237

E.1 Frequentist methods compared. MiniBooNE BDT analysis. . . . . . . 242 
E.2 Frequentist Methods Compared. BDT analysis (II) . . . . . . . . . 243

E.3 Effective number of degrees of freedom, $20 \times 20$ grid. . . . . . . . 244

F.1 Testing statistical correlations. Fake data fit, all errors (BDT). . . . 247

F.2 Testing statistical correlations. Fake data fit, all errors (TBL) . . . . 248

F.3 Testing statistical correlations. Fake data fit, all errors (BDT, unsmoothed O.M.). . . . . . . . . . . . . . . . . . . . . . 249

F.4 Testing statistical correlations. Fake data fit, all errors (TBL, unsmoothed O.M.). . . . . . . . . . . . . . . . . 250

F.5 Slices of the raster-scan $\Delta \chi^{2}$ surface. Sensitivity. . . . . . . . . 252

F.6 Slices of the raster-scan $\Delta \chi^{2}$ surface. Data fit. . . . . . . . . 253

F.7 Fitting a fake data set with a strong signal. . . . . . . . . 257

F.8 Fitting a fake data set with no oscillations signal. . . . . . . . 258

F.9 Fake fit to a strong signal, Combined BDT $+\mathrm{TBL}+\nu_{\mu}$ fit. . . . . . . 259

F.10 Fake fit to a null signal, Combined BDT $+\mathrm{TBL}+\nu_{\mu}$ fit. $\ldots \ldots \ldots 260$

G.1 Path of NuMI trigger signals along communications ducts. . . . . . 263

G.2 Time distribution of neutrino candidates from the NuMI beam in Mini-

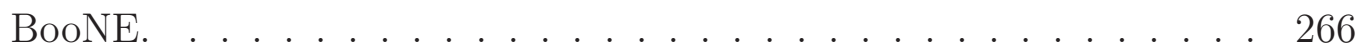

G.3 Rate of NuMI neutrino candidates as a function of time. . . . . . 268

G.4 Distributions for NuMI events exiting the tank. . . . . . . . . . 270

G.5 Illustration of the random throw of neutrino vectors, NuMI event simulation. . . . . . . . . . . . . . . . . . . . . 272

G.6 Predicted neutrino flux from the NuMI beamline at MiniBooNE. . . . 275 
G.7 Predicted neutrino flux from the NuMI beamline at MiniBooNE. . . . 276

H.1 GEANT4 rendering of the NuMI and MiniBooNE beamlines. . . . . . 285

H.2 Schematic of the NuMI and MiniBooNE beamlines. . . . . . . . . . 286 


\section{Acknowledgments}

I would like to express my most sincere thanks to my Ph.D. advisor, Prof. Mike Shaevitz for his support, guidance, and patience during my years at Columbia. I am also grateful to Prof. Janet Conrad for her support, and for her leadership and strong determination as co-spokesperson of the MiniBooNE experiment.

I thank all of the MiniBooNE collaborators, whose hard work made possible the publication of the experiment's first oscillations result. The work on this thesis would not have been possible without all the individual contributions of this amazing group of people.

In particular, I thank Dave Schmitz, fellow Columbia University graduate student, for taking on the BDT oscillations analysis before and during the box opening days, and for his friendship and generosity in providing me with many of the tools that were instrumental for this work. I thank Columbia University post-doc Zelimir Djurcic for being a great neighbor and friend, and also for taking on the analysis of NuMI events in MiniBooNE and turning it into a great piece or work. To the former graduate students in the Columbia University Neutrino Group: Michel Sorel, Jocelyn Monroe, thanks for the help and guidance you gave me during the time we worked together. To my fellow Columbia University graduate students at Fermilab: Kendall Mahn, Georgia Karagiorgi, and Van Nguyen, thank you for your generous friendship and unending enthusiasm, and for the countless helpful and interesting discussions that we had. I 
thank MiniBooNE's co-spokesperson Steve Brice, for teaching me nearly everything I know about calculating error matrices, and for always finding time to answer my questions. For their input and help with the review of the analysis presented in this thesis I thank Byron Roe, Ion Stancu, and Chris Polly.

Being a graduate student at Fermilab would not have been so much fun without friends like Teppei Katori, Serge Ouedraogo, Denis Perevalov, and Yong Liu. To all of them I thank for their companionship, sense of humor, and help in all sorts of matters.

None of this could have been possible without the help of Hywel White and Geoff Mills, who mentored me as an undergraduate for a few months at Los Alamos working on the LSND experiment, and first suggested me to join MiniBooNE. During those days at Los Alamos I met Rex Tayloe, Gerry Garvey, Richard Van de Water, and Bill Louis, who have been a source of inspiration and encouragement during my years as a graduate student. To all of them, thanks.

I thank my parents, Alexis Aguilar and Dora Arévalo, who have always been supportive of my endeavors, and very specially to Graciela Canseco, whose constant love and support maintained me on course to reach this goal. 


\section{Introduction}

Neutrinos were postulated by W. Pauli in 1930 [1] to explain the continuous energy spectrum of the electrons emitted in the beta decay of certain radioactive nuclei while maintaining energy and momentum conservation. Pauli originally named this new particle neutron, and determined that besides being electrically neutral, it should also have spin $1 / 2$ and a very small mass (at most $10^{-2}$ times that of the proton). Pauli's neutron would exist in the interior of atomic nuclei, thought at the time to be composed by protons and electrons, and in a beta decay would be emitted together with an electron in a three body decay explaining the continuous energy spectrum of the electrons.

Pauli's idea gained acceptance with the discovery of the neutron as a constituent of atomic nuclei by Chadwick in 1932 [2], and in 1934 Fermi used it to complete his theory of beta decay [3], giving it the name neutrino to distinguish it from Chadwick's neutron. In 1956 Reines and Cowan [4] first detected the neutrinos from a nuclear reactor through the reaction known as inverse beta decay $\left(p+\bar{\nu}_{e} \rightarrow n+e^{+}\right)$, and in 1962 the Columbia University group led by Lederman, Schwartz and Steinberger [5] discovered the muon neutrino. The discovery of the tau lepton in 1975 [6] suggested the existence of a third neutrino, the $\nu_{\tau}$, pointing to the scheme of three generations of particles in the Standard Model completed in 1995 [7] with the discovery of the top quark in Fermilab in 1995. 
The idea of neutrino oscillations was first proposed by Pontecorvo in 1957 [8] suggesting the possibility of $\nu \leftrightarrow \bar{\nu}$ transitions in analogy with $K^{0} \bar{K}^{0}$ oscillations [9]. Soon after the discovery of the muon neutrino, Maki, Nakagawa and Sakata [10] suggested that transitions between neutrinos of different flavors could occur if the neutrinos were massive particles and if the states with definite flavor and definite mass were related to one another by a linear transformation similar to a change of basis. This idea provided a framework to interpret the early observations of the deficit of solar neutrinos made by Ray Davis in the 60's and 70's, which admitted neutrino flavor transitions as a possible solution. However, it was not supported by the lack of evidence for oscillations coming from pioneering experiments using nuclear reactors.

In the mid 1990's the LSND experiment at Los Alamos National Laboratory searched for neutrino oscillations of the type $\bar{\nu}_{\mu} \rightarrow \bar{\nu}_{e}$ with a neutrino beam that traveled a short distance $(\sim 30 \mathrm{~m})$ from source to detector, finding a positive signal in favor of this process. Soon after in 1998, the Super-Kamiokande experiment [65], in Japan presented for the first time strong evidence in favor of neutrino oscillations of the type $\nu_{\mu} \rightarrow \nu_{\tau}$ from the observation of muon neutrinos produced in the Earth's atmosphere traveling distances comparable to the Earth's diameter. The observations implied three very distinct regimes of oscillations requiring the existence of at least one new type of neutrino, in conflict with the three-neutrino picture of the Standard Model. New experiments were performed and the signatures for oscillations from solar and atmospheric neutrinos were confirmed, while the LSND observation remained unverified.

Recent experimental observations of solar, atmospheric, and accelerator neutrino oscillations (see Section 1.4, Chapter 1) have conclusively established that neutrinos have non-zero masses. In order to seriously address the LSND anomaly, as it became known, the MiniBooNE experiment at Fermilab performed a search for $\nu_{\mu} \rightarrow \nu_{e}$ oscil- 
lations in the same region of the oscillations parameter space that would account for the LSND observation. First results from this search have been published providing evidence that the LSND result cannot be due to the simple two-neutrino oscillations model as was originally thought. Two different analyses of the MiniBooNE data based on different particle identification algorithms and techniques to reduce the effect of systematic uncertainties gave consistent answers disfavoring the two-neutrino oscillation hypothesis. A first attempt to combine the power of the two analyses is the goal of this thesis where a combination of the two particle identification methods is used to explore the gain in the oscillation sensitivity and in the exclusion power of the final fit to the MiniBooNE data.

The results of this thesis are summarized in Figures 1, 2, and 3 below. In Figure 1 we show the fits to the energy distributions of the two $\nu_{e}$ samples, obtained with the two particle identification methods (labeled BDT and TBL in the figures), and the $\nu_{\mu}$ sample (see Chapters 4 and 5 for the full description of these results) with the technique of this thesis. In Figure 2 we compare the oscillations results ${ }^{1}$ obtained using both $\nu_{e}$ samples to those obtained using either of the two $\nu_{e}$ samples separately ${ }^{2}$. In Figure 3 we compare the results with the previously published ones, where it is shown that there is a net gain in sensitivity to oscillations (Figure 2(a)), as well as an increase in the exclusion limit below $\Delta m^{2} \lesssim 1 \mathrm{eV}^{2}$ when compared with the limit previously published by the collaboration [190] (Figure 3(b)).

\section{Thesis Results in next three pages.}

\footnotetext{
${ }^{1}$ Sensitivity to $2 \nu$ oscillations of the LSND type and limits on oscillations parameters, drawn as curves on the $\Delta m^{2}$ vs. $\sin ^{2} 2 \theta$ plane introduced in Section 1.2.1.

${ }^{2}$ Also shown are the $90 \%$ (dark filled area) and 99\% (light filled area) C.L. allowed regions of LSND which will appear in all similar plots of the $\Delta m^{2} v s . \sin ^{2} 2 \theta$ plane throughout this thesis.
} 


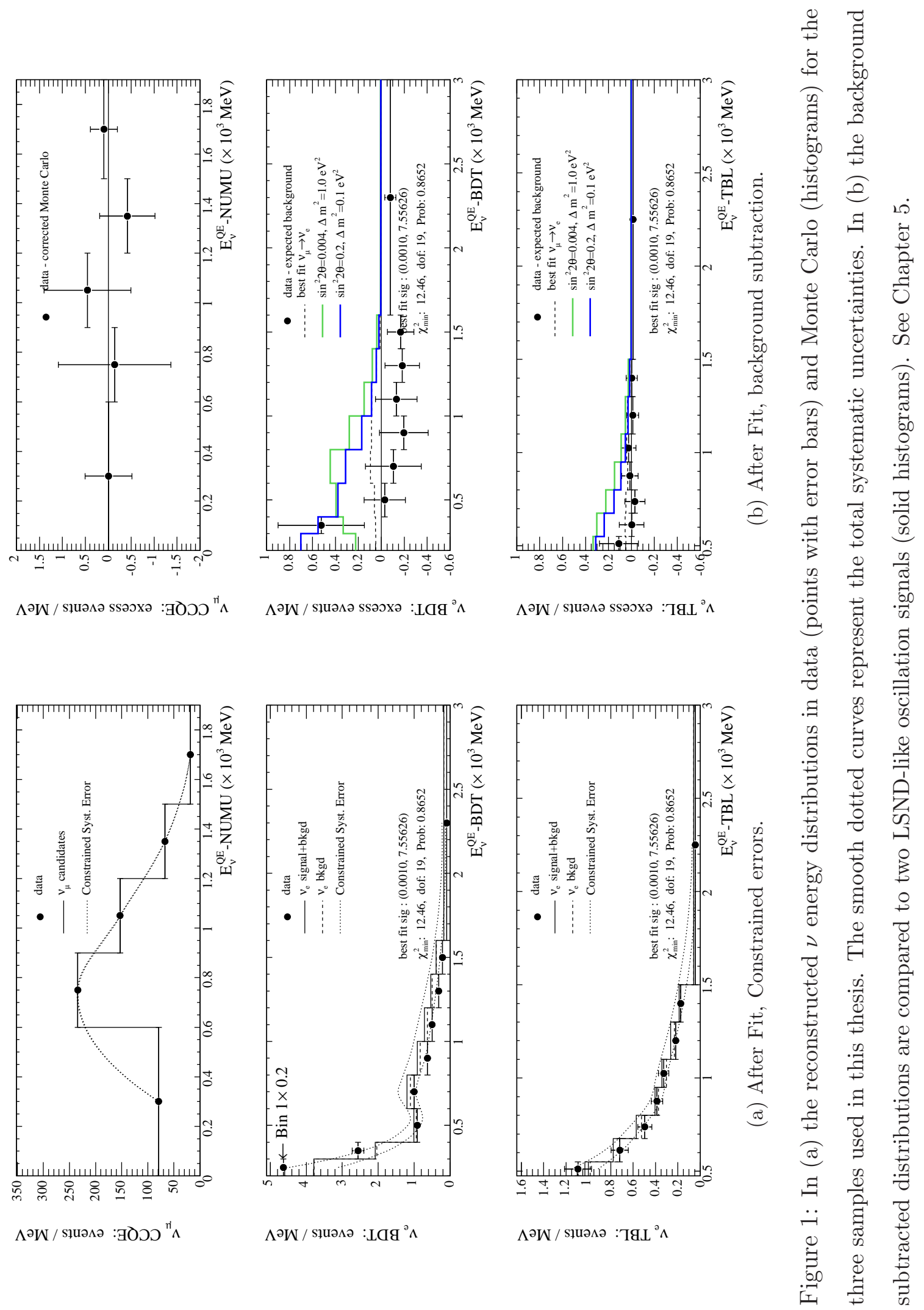




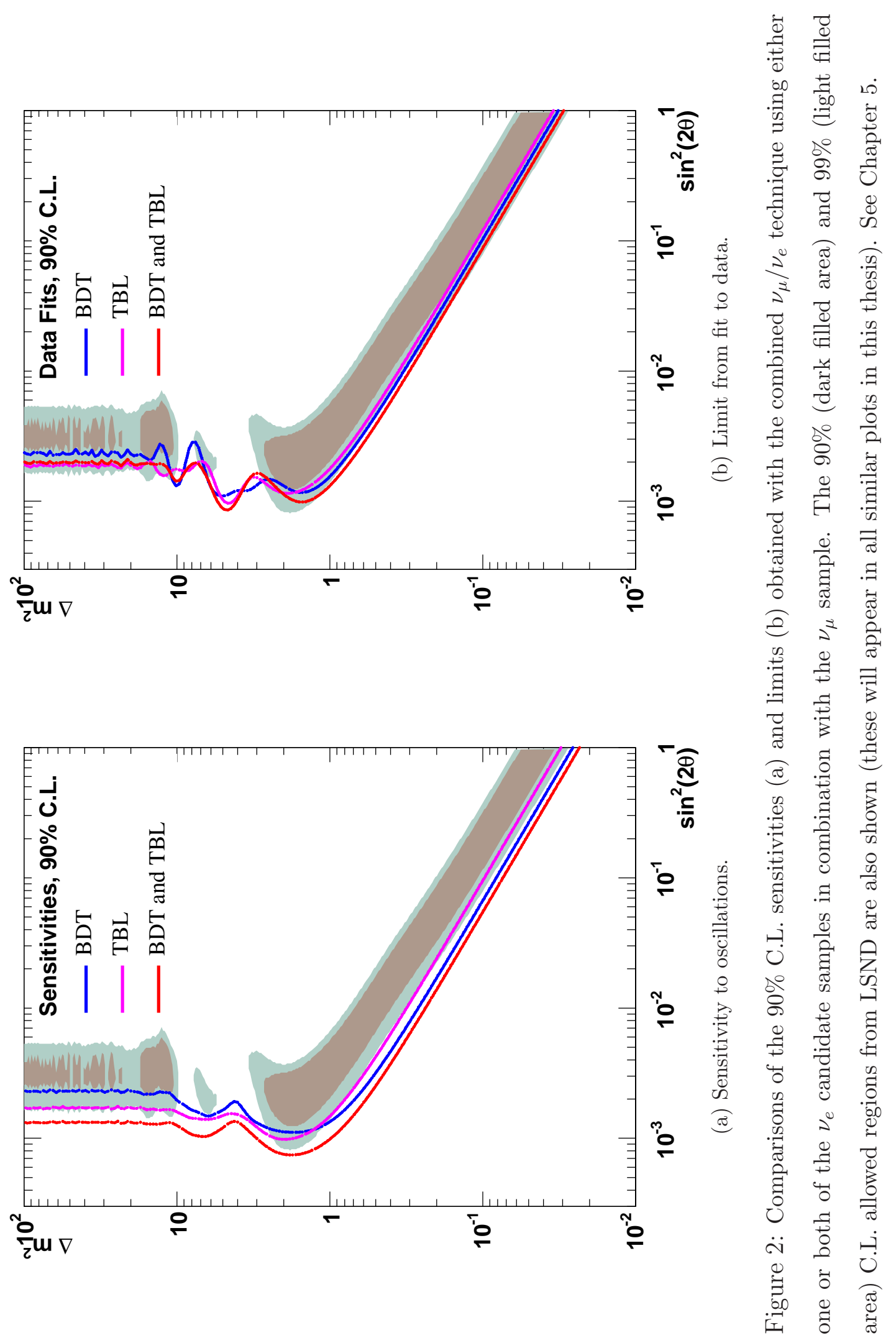




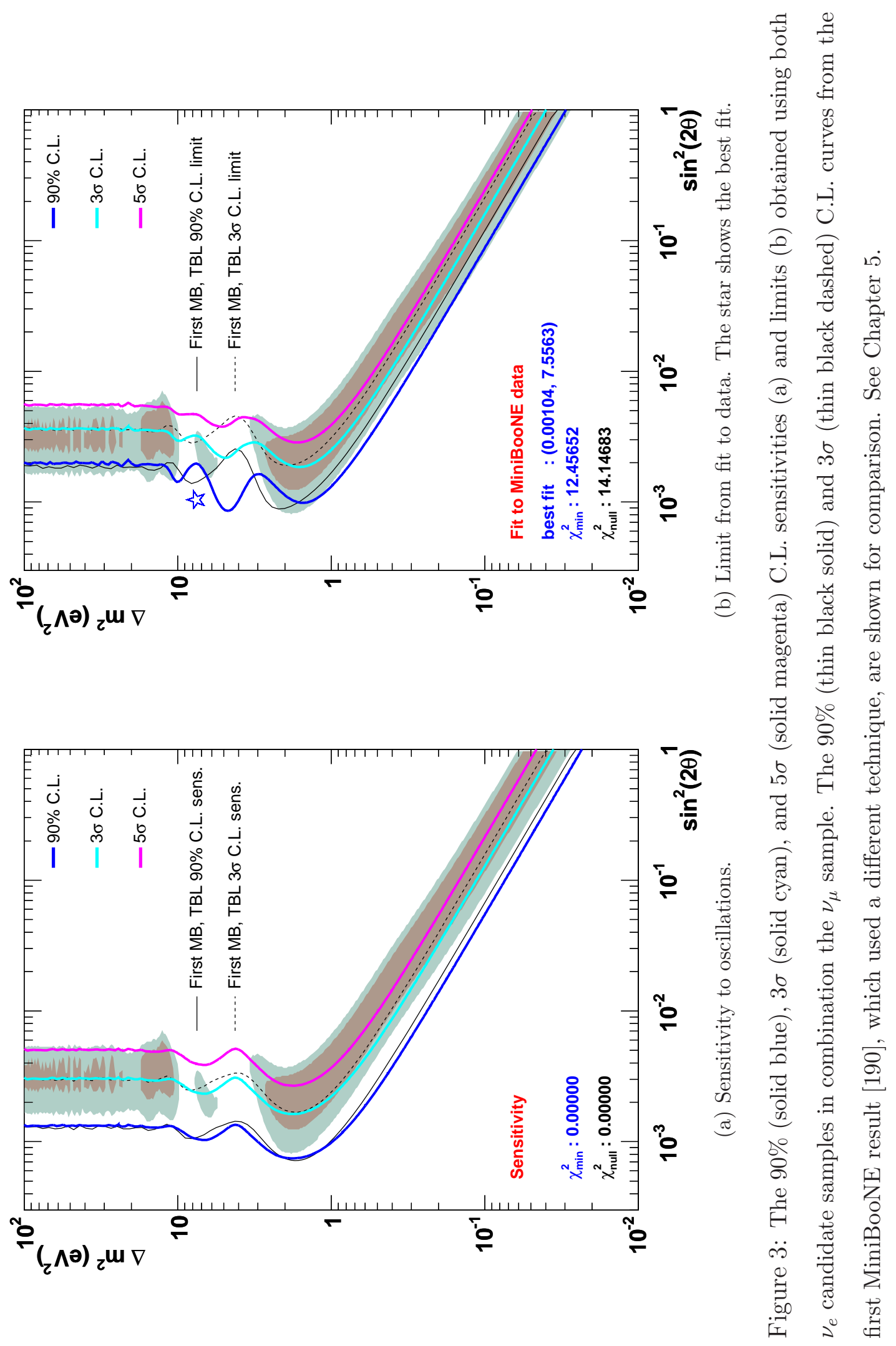


In Chapter 1 a brief description of the theory of massive neutrinos and neutrino oscillations is given, followed by a compilation of results from experiments on neutrino oscillations including LSND which is the main motivation for the MiniBooNE experiment. In Chapter 2 the MiniBooNE experimental apparatus and the subsystems relevant for the present work are described. In Chapter 3 we describe the experiment simulation, reconstruction algorithms, particle identification methods, and the estimation of systematic uncertainties for the $\nu_{\mu} \rightarrow \nu_{e}$ oscillations search. In this chapter we also introduce the boosted decision tree (BDT) and track based likelihood (TBL) criteria used to select $\nu_{e}$ candidate samples for the oscillations search, as well as the criterion used to select a high statistics $\nu_{\mu}$ candidate sample that helps constrain the effect of systematic uncertainties.

The original contributions from this thesis are presented in Chapter 4 where the technique to combine the BDT and TBL $\nu_{e}$ candidate samples is described in detail, and in Chapter 5 where the results and conclusions of the application of the proposed technique are compared to those previously made public by the collaboration. 


\section{Chapter 1}

\section{Neutrinos}

\subsection{Field Theory of massive fermions}

A free fermion of mass $m$ is described by 4-component spinor field $\psi$ satisfying the Dirac equation:

$$
\left(i \gamma^{\mu} \partial_{\mu}-m\right) \psi=0
$$

where $\gamma^{i}, i=0,1,2,3$ are the Dirac matrices [19]. Using the Euler-Lagrange equation $\partial_{\mu}\left(\partial \mathcal{L} / \partial\left(\partial_{\mu} \bar{\psi}\right)\right)=\partial \mathcal{L} / \partial \bar{\psi}$ one can derive Eq.(1.1) from the Dirac Lagrangian density $\mathcal{L}=\bar{\psi}\left(i \gamma^{\mu} \partial_{\mu}-m\right) \psi$. The projections $\psi_{L}=1 / 2\left(1-\gamma^{5}\right) \psi$ and $\psi_{R}=1 / 2\left(1+\gamma^{5}\right) \psi$ are eigenstates of $\gamma^{5} \equiv i \gamma^{0} \gamma^{1} \gamma^{2} \gamma^{3}$, which satisfies $\left(\gamma^{5}\right)^{2}=1$. They are called the left $(\mathrm{L})$ and right $(\mathrm{R})$ handed components of $\psi=\left(\psi_{L}+\psi_{R}\right)$ and are said to have definite chirality. The evolution described by the Dirac equation mixes the left and right handed components of the field, but the anti commutation relation $\left\{\gamma^{5}, \gamma^{\mu}\right\}=0$ guarantees that in the ultra-relativistic limit $E / m \rightarrow \infty$ (or for massless particles) the definite chirality states $\psi_{L}$ and $\psi_{R}$ satisfy the Dirac equation at all times making chirality (also called handedness) a good quantum number. In this limit the projection of the particle's spin $\Sigma=-i \gamma^{4} \gamma^{5} \gamma$ along its momentum p, called helicity, satisfies 
$\boldsymbol{\Sigma} \cdot \mathbf{p} /|\mathbf{p}| \rightarrow \gamma^{5}$. Therefore, helicity and chirality are the same for massless particles.

\section{Massless neutrinos in the Standard Model}

In the Standard Model neutrinos are massless particles that interact with matter only through the weak interaction ${ }^{1}$. They can interact via charged current (CC) interactions (exchange of a $W^{+}$or $W^{-}$boson) or neutral current (NC) interactions (exchange of a $Z^{0}$ boson). The particles of the three generations are arranged into two $\mathrm{SU}(2)_{L}$ weak isospin doublets and three weak isospin singlets as shown in Table 1.1

Table 1.1: Left handed isospin doublets and right handed isospin singlets in the Standard Model. In the Table $\psi$ represents the neutrino field $\nu$.

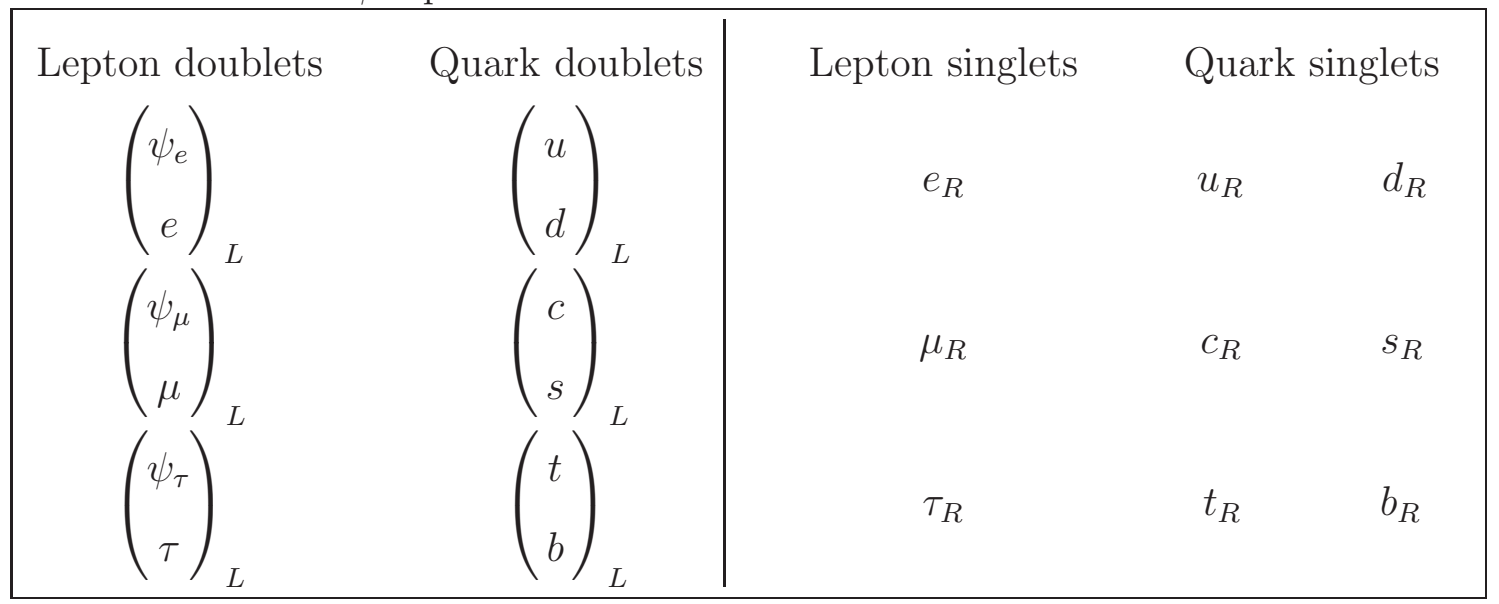

A Higgs boson doublet $\left(\phi^{+}, \phi\right)^{T}$ with a non-zero vacuum expectation value (VEV) $(0, v / \sqrt{2})^{T}$ is responsible for breaking the $\mathrm{SU}(2)_{L}$ symmetry giving masses to the gauge bosons $W^{ \pm}$and $Z^{0}$ as well as the massive fermions. The weak interactions are restricted to involve exclusively the left handed components of the interacting

\footnotetext{
${ }^{1}$ Massless neutrinos also feel the effects of gravitational fields, however, gravity is not a part of the Standard Model of Particle Physics and is hence excluded from the discussion.
} 
particles, hence the $\mathrm{CC}$ and $\mathrm{NC}$ interactions are described by terms in the Lagrangian of the form [19]:

$-\mathcal{L}_{C C}=\frac{g}{\sqrt{2}} \sum_{l=e, \mu, \tau} \bar{\psi}_{l L} \gamma^{\mu} l_{L} W^{+}+$h.c.,$\quad-\mathcal{L}_{N C}=\frac{g}{\sqrt{2 \cos \theta_{W}}} \sum_{l=e, \mu, \tau} \bar{\psi}_{l L} \gamma^{\mu} l_{L} W^{+}+$h.c. where $g$ is the the $\mathrm{SU}(2)_{L}$ coupling constant, and $\theta_{W}$ is the Weinberg angle. Such terms will appear in the Lagrangian upon the imposition of local $\mathrm{SU}(2)_{L} \times \mathrm{U}(1)_{Y}$ gauge invariance and bring interactions into the theory. The SM contains no right handed neutrino fields making impossible the existence of a Dirac mass term. This in turn causes the neutrino magnetic moment and mixings to vanish [22]. However, there is no fundamental symmetry in the model that prevent us from including right handed fields in one way or another [23].

To the present day, there has been no observation of a process consistent with a neutral current interaction that involves a net change in flavor. Many experiments have looked for processes such as $K_{L}^{0} \rightarrow e^{+} e^{-}, K_{L}^{0} \rightarrow \mu^{+} \mu^{-}, K_{L}^{0} \rightarrow \mu^{ \pm} e^{\mp}, K^{+} \rightarrow$ $\pi^{+} \nu \bar{\nu}, \mu \rightarrow e \gamma, \mu^{ \pm} \rightarrow e^{-} e^{+} e^{-}, \mu+$ Nucl $\rightarrow e+$ Nucl, etc., placing limits on the strength of such flavor changing neutral current (FCNC) interactions to the level of $\sim 10^{-4} \times G_{F}$ from Kaon decays, and $\sim 10^{-6} \times G_{F}$ from muon decays [11]. These observations imply that one can define lepton family quantum numbers $L_{e}, L_{\mu}$, and $L_{\tau}$, which are conserved in weak interactions. The analogous family numbers for quarks are not possible because of the observed flavor change in charged current weak interactions, manifested through the CKM mixing matrix in the quark sector. This makes charged leptons and neutrinos very different from quarks.

\section{Neutrino mass terms}

It is possible to construct states with definite chirality that are different from $\psi_{L}$ and $\psi_{R}$ by means of the charge conjugation operator $C$. Defining the charge conjugate 
field $\psi^{c}=\eta_{C} C \bar{\psi}^{T}$ where $\eta_{C}$ is a phase factor, $\bar{\psi}=\psi^{\dagger} \gamma^{0}$ is the Dirac adjoint of field $\psi$, and $T$ represents the transpose, one can form the states [20]

$$
\left(\psi_{L}\right)^{c}=\frac{1+\gamma^{5}}{2} \psi^{c} \equiv\left(\psi^{c}\right)_{R} \quad \text { and } \quad\left(\psi_{R}\right)^{c}=\frac{1-\gamma^{5}}{2} \psi^{c} \equiv\left(\psi^{c}\right)_{L}
$$

which are also eigenstates of $\gamma^{5}$ and therefore have definite chirality. In terms of the chiral fields, the mass term in the Dirac Lagrangian is $m \bar{\psi} \psi=m\left(\bar{\psi}_{R} \psi_{L}+\bar{\psi}_{L} \psi_{R}\right)$. This term is only one of many possible terms satisfying Lorentz invariance and being hermitian. A more general Lagrangian density for a massive particle will admit terms such as $\bar{\psi}_{L} \psi_{R}, \quad \overline{\psi^{c}}{ }_{R} \psi_{L}=\overline{\left(\psi_{L}\right)^{c}} \psi_{L}, \quad \overline{\psi^{c}}{ }_{L} \psi_{R}=\overline{\left(\psi_{R}\right)^{c}} \psi_{R}$, and their hermitian conjugates [21]. These terms can be introduced with three real masses $M_{D}, M_{L}$, and $M_{R}$ to form the most general mass Lagrangian density respecting CP invariance ${ }^{2}$ :

$$
-\mathcal{L}_{\text {mass }}=M_{D}\left(\bar{\psi}_{L} \psi_{R}+\text { h.c. }\right)+\frac{M_{L}}{2}\left(\overline{\left(\psi_{L}\right)^{c}} \psi_{L}+\text { h.c. }\right)+\frac{M_{R}}{2}\left(\overline{\left(\psi_{R}\right)^{c}} \psi_{R}+\text { h.c. }\right) .
$$

The first term is the usual mass term of a Dirac field, and the next two terms are called Majorana mass terms. If we define the Majorana fields

$$
\phi \equiv \frac{\psi_{L}+\left(\psi_{L}\right)^{c}}{\sqrt{2}} \quad \text { and } \quad \Phi \equiv \frac{\psi_{R}+\left(\psi_{R}\right)^{c}}{\sqrt{2}}
$$

the mass term takes the simple form $-\mathcal{L}_{\text {mass }}=M_{D}(\bar{\phi} \Phi+\bar{\Phi} \phi)+M_{L} \bar{\phi} \phi+M_{R} \bar{\Phi} \Phi$. Adding one kinetic term for each of these two new fields and arranging the mass constants into a matrix $\mathbf{M}$, the total Lagrangian density of the system can be written, using matrix notation for the mass term, as

$$
-\mathcal{L}=-\bar{\phi} i \gamma^{\mu} \partial_{\mu} \phi-\bar{\Phi} i \gamma^{\mu} \partial_{\mu} \Phi+(\bar{\phi}, \bar{\Phi})\left(\begin{array}{cc}
M_{L} & M_{D} \\
M_{D} & M_{R}
\end{array}\right)\left(\begin{array}{l}
\phi \\
\Phi
\end{array}\right)
$$

The matrix in the above expression is called the mass matrix of the system. If $\nu^{\prime}$ and $N$ are the eigenvectors of the mass matrix, with eigenvalues $M_{\nu^{\prime}}$ and $M_{N}$ respectively,

\footnotetext{
${ }^{2}$ It can be shown that for the case of complex constants, the Lagrangian is not CP invariant[20].
} 
the Lagrangian of the system is expressed as

$$
-\mathcal{L}=-\overline{\nu^{\prime}} i \gamma^{\mu} \partial_{\mu} \nu^{\prime}-\bar{N} i \gamma^{\mu} \partial_{\mu} N+M_{\nu^{\prime}} \overline{\nu^{\prime}} \nu+M_{N} \bar{N} N
$$

which is the free Lagrangian density of two spin $1 / 2$ particles $\nu^{\prime}$ and $N$, with masses $M_{\nu^{\prime}}$ and $M_{N}$. The Dirac field $\psi$ describes the two spin states of a particle and its antiparticle having the same mass (four states). Introduction of the Majorana mass terms breaks the degeneracy giving a different mass to the fields $\nu^{\prime}$ and $N$. Given that the spinor fields can only represent four different states, these fields represent particles that are identical to their antiparticles (CPT self-conjugate).

Pairing left and right-handed components of a field with its Dirac adjoint, Dirac mass terms explicitly conserve electric charge and can be used to describe charged massive particles. Majorana mass terms on the other hand can only exist for electrically neutral particles, otherwise one would allow for violation of charge conservation by two units. Therefore the charged leptons admit a description in terms of Dirac, but no Majorana mass terms.

\section{The see-saw mechanism}

A simple extension of the standard model that allows neutrino masses is to include right handed neutrino fields $\psi_{R}$ as members of an $\mathrm{SU}(2)_{R}$ doublet analogous to the $\mathrm{SU}(2)_{L}$ doublets already present in the theory. For the first family one would have

$$
\left(\begin{array}{c}
\psi_{e} \\
e
\end{array}\right)_{L}\left(\begin{array}{l}
\psi_{e} \\
e
\end{array}\right)_{R}
$$

where $e=e_{L}+e_{R}$ represent the electron field and its chiral components arranged in their corresponding doublets. Such a theory would have an underlying $\mathrm{SU}(2)_{L} \times$ $\mathrm{SU}(2)_{R} \times \mathrm{U}(1)$ symmetry which spontaneously breaks at two different energy scales. The mass terms for the neutrino fields will arise from the Yukawa couplings of the 
fields in the doublets with scalar Higgs fields which acquire some vacuum expectation value (VEV) when the symmetry is broken. To obtain the mass terms in Eq.(1.3) one can introduce three scalar Higgs fields $h, h_{L}$, and $h_{R}$ with appropriate weak isospin quantum numbers to guarantee the gauge invariance of the Lagrangian under $\mathrm{SU}(2)_{L} \times \mathrm{SU}(2)_{R} \times \mathrm{U}(1)$ transformations [21]. The Yukawa couplings will be of the form $\bar{\psi}_{R} h \psi_{L}, \overline{\left(\psi_{L}\right)^{c}} h_{L} \psi_{L}, \overline{\left(\psi_{R}\right)^{c}} h_{R} \psi_{R}$, and the masses in the general Lagrangian density will then be $M_{D} \sim\langle h\rangle, M_{L} \sim\left\langle h_{L}\right\rangle, M_{R} \sim\left\langle h_{r}\right\rangle$, where the brackets $\langle\cdot\rangle$ represent the VEV. It is reasonable to assume that $\left\langle h_{L}\right\rangle \approx 0$, since a non-zero value for this VEV would affect the relative strengths of the $\mathrm{CC}$ and NC interactions, found experimentally to be approximately equal [19]. One can also assume that $\left\langle h_{R}\right\rangle>>\langle h\rangle$ which is true if the scale of the $\mathrm{SU}(2)_{L}$ symmetry breaking is much larger than that of the $\mathrm{SU}(2)_{L}$ electroweak symmetry, occurring around the mass of the $W^{ \pm}$boson of $\sim 83 \mathrm{GeV}$. Furthermore, the field $h$ will continue to be responsible to give the other fermions in the SM their masses, and we can expect that $M_{D}$ be of order the mass of the quarks or leptons. In this situation, the mass matrix for the neutrinos becomes

$$
\mathbf{M} \approx\left(\begin{array}{cc}
0 & M_{D} \\
M_{D} & M_{R}
\end{array}\right)
$$

and the eigenvectors and eigenvalues are given in terms of the Majorana fields by

$$
\begin{aligned}
& N \approx \Phi+\frac{M_{D}}{M_{R}} \phi, \quad M_{N} \approx M_{R}, \\
& \nu^{\prime} \approx \phi-\frac{M_{D}}{M_{R}} \Phi, \quad M_{\nu^{\prime}} \approx-\frac{M_{D}^{2}}{M_{R}} .
\end{aligned}
$$

To get a positive eigenvalue for the light neutrino state we take the physical neutrino field to be $\nu=\gamma^{5} \nu^{\prime}$, which will satisfy $\overline{\nu^{\prime}} \nu^{\prime}=-\bar{\nu} \nu$ and $\overline{\nu^{\prime}} \gamma_{\mu} \partial_{\mu} \nu^{\prime}=+\bar{\nu} \gamma_{\mu} \partial_{\mu} \nu$. This gives the Lagrangian density for the free neutrinos $\nu$ and $N$ and their masses

$$
\begin{gathered}
-\mathcal{L}=-\bar{\nu} i \gamma^{\mu} \partial_{\mu} \nu+M_{\nu} \bar{\nu} \nu-\bar{N} i \gamma^{\mu} \partial_{\mu} N+M_{N} \bar{N} N . \\
M_{N} \approx M_{R} \text { and } M_{\nu} \approx+\frac{M_{D}^{2}}{M_{R}}
\end{gathered}
$$


Under the assumption that $M_{D} \sim M_{l \text { or } q}$ (the mass of a quark or lepton in the $\mathrm{SM}$ ), and the existence of a heavy neutrino state $N$, this procedure gives a natural explanation as to why neutrino masses are so small. The relation $M_{\nu} \times M_{N} \approx M_{l \text { or } q}^{2}$ is known as the see-saw relation.

\section{$1.2 \quad$ Neutrino mixing}

If neutrinos have masses, there is a set of at least three definite mass states, usually denoted by $\nu_{1}, \nu_{2}, \nu_{3}, \ldots, \nu_{n}$ which may in general be different from the definite-flavor weak interaction eigenstates $\nu_{e}, \nu_{\mu}$, and $\nu_{\tau}$. We use the term mixing to refer to the situation in which the weak interaction couples any charged-lepton mass eigenstate $l_{\alpha}$, $\alpha=e, \mu, \tau$, with any of the neutrino mass eigenstates $\nu_{i}$. Then the various amplitudes ${ }^{3}$ for the decay of a charged $W^{ \pm}$boson into the different pairings of charged leptons and neutrino mass eigenstates can be arranged into the elements of a $3 \times n$ complexvalued unitary matrix $U$ known as the leptonic mixing matrix [10]. For example, the amplitude for the decay of a $W^{+}$into the combination $l_{\alpha}^{+}+\nu_{i}$ is the element $U_{\alpha i}^{*}$ of the complex conjugate matrix $U^{*}$. The neutrino state created in the decay $W^{+} \rightarrow l_{\alpha}^{+}+\nu$ can be seen as a superposition of definite-mass eigenstates weighted by the amplitudes of each transition connecting them to the charged lepton $\alpha$ :

$$
\left|\nu_{\alpha}\right\rangle=\sum_{i} U_{\alpha i}^{*}\left|\nu_{i}\right\rangle
$$

We refer to this superposition as the neutrino state of definite flavor $\alpha$, and write from now on the $W^{+}$decay reaction in the form $W^{+} \rightarrow l_{\alpha}^{+}+\nu_{\alpha}$

For cases in which the number of mass eigenstates is equal to the number of flavor eigenstates, the leptonic mixing matrix can be thought of as a rotation matrix taking

\footnotetext{
3 Their complex conjugates, by convention.
} 
us from the definite flavor basis to the definite mass basis. This matrix can be formed by the consecutive application of rotations about the definite mass axes $\nu_{1}, \nu_{2}$, and $\nu_{3}$ and parameterized by three angles $\theta_{12}, \theta_{23}$, and $\theta_{23}$ giving the magnitude of each of these rotations about the $\nu_{3}, \nu_{1}$, and $\nu_{2}$ axes respectively.

The most general form of the neutrino mixing matrix for three neutrinos is the $\mathrm{PMNS}^{4}$ matrix:

$$
\nu_{\mu} \nu_{\nu_{\tau}}\left[\begin{array}{ccc}
\nu_{1} & \nu_{2} & \nu_{3} \\
\nu_{12} c_{13} & s_{12} c_{13} & s_{13} e^{-i \delta} \\
-s_{12} c_{23}-c_{12} s_{23} s_{13} e^{i \delta} & c_{12} c_{23}-s_{12} s_{23} s_{13} e^{i \delta} & s_{23} c_{13} \\
s_{12} s_{23}-c_{12} c_{23} s_{13} e^{i \delta} & -c_{12} s_{23}-s_{12} c_{23} s_{13} e^{i \delta} & c_{23} c_{13}
\end{array}\right]
$$

where $c_{i j} \equiv \cos \theta_{i j}$ and $s_{i j} \equiv \sin \theta_{i j}$, and $\delta$ is the CP-violating phase. The phases $\alpha_{1}$ and $\alpha_{2}$ are called Majorana phases, and can potentially appear in the case neutrinos are their own anti particles. For Dirac neutrinos these phases can be absorbed in a redefinition of the Dirac fields.

\section{Sterile Neutrinos}

If the number of mass eigenstates is larger than the number of weak interaction eigenstates there are more linearly independent superpositions of mass eigenstates than there are neutrino flavors. Let these extra states be denoted by

$$
\left|\nu_{s}\right\rangle=\sum_{i} U_{s i}^{*}\left|\nu_{i}\right\rangle, s \neq e, \mu, \tau
$$

Such states will not couple to the Standard Model $W^{ \pm}$bosons, since they do not

\footnotetext{
${ }^{4}$ Pontecorvo-Maki-Nakagawa-Sakata [11].
} 
have a corresponding charged lepton partner in the model. There is strong experimental evidence that the number of neutrinos participating in the decays $Z \rightarrow \nu_{\alpha} \bar{\nu}_{\alpha}$ of the $Z$ boson is almost exactly equal to three [11], indicating that if they exist, these linear superpositions do not couple to the $Z$ boson either. Having no interactions with any of the Standard Model bosons, these states are called sterile neutrinos.

Both, neutrino masses and mixing are not contained in the Standard Model of Particle Physics, and are necessary for the phenomenon of neutrino oscillations to occur.

\subsubsection{Neutrino oscillations in vacuum}

The time evolution of the neutrino state in Eq.(1.5) can be determined by calculating the evolution of its definite mass components:

$$
\left|\nu_{\alpha}(t)\right\rangle=\sum_{i}^{n} U_{\alpha i}^{*}\left|\nu_{i}(t)\right\rangle
$$

Approximating each neutrino mass eigenstate by a plane wave, its time evolution is given by ${ }^{5}:\left|\nu_{i}(t)\right\rangle=e^{-i E_{i} t}\left|\nu_{i}(0)\right\rangle$, where $E_{i}=\sqrt{p_{i}^{2}+m_{i}^{2}}$ is the energy of the state of mass $m_{i}$ and momentum $p_{i}$. A useful assumption that is made when working with the plane wave approximation used here is that all the mass eigenstate components are created with the same momentum $p_{i} \approx p$, yielding a flavor eigenstate with a definite momentum (plane wave with momentum $p$ ). The alternate assumption [21] that the mass eigenstates have the same energy instead leads to the same expression for the flavor transition probability. Furthermore, the neutrino will be highly relativistic and its momentum will be much larger than the masses of the mass eigenstates. Under these assumptions, we approximate the energy of the mass-eigenstate $\nu_{i}$ by $E_{i}=\sqrt{p^{2}+m_{i}^{2}} \approx p+m_{i}^{2} / 2 p$.

\footnotetext{
${ }^{5}$ In this section we use natural units in which $c=\hbar=1$.
} 
The probability for a transition from the flavor state $\alpha$ to the flavor state $\beta$ to occur at time $t$ will be given by

$$
P_{\alpha \beta}=\left|\left\langle\nu_{\beta} \mid \nu_{\alpha}(t)\right\rangle\right|^{2}=\left|\sum_{i=1}^{n} \sum_{j=1}^{n} U_{\alpha i}^{*} U_{\beta j}\left\langle\nu_{j} \mid \nu_{i}(t)\right\rangle\right|^{2}
$$

Using the orthonormality condition $\left\langle\nu_{j} \mid \nu_{i}\right\rangle=\delta_{i j}$, and setting the time $t=L / c$, the distance traveled by the neutrino from the production point to the detection point divided by its speed (approximately equal to the speed of light $c$ ), one arrives at the general formula for the transition probability between two flavor states:

$$
\begin{aligned}
P\left(\nu_{\alpha} \rightarrow\right. & \left.\nu_{\beta}\right)=\delta_{\alpha \beta} \\
& -4 \sum_{i>j} \mathcal{R}\left(U_{\alpha i}^{*} U_{\beta_{i}} U_{\alpha j} U_{\beta_{j}}^{*}\right) \sin ^{2}\left[1.27 \Delta m_{i j}^{2}(L / E)\right] \\
& +2 \sum_{i>j} \mathcal{I}\left(U_{\alpha i}^{*} U_{\beta_{i}} U_{\alpha j} U_{\beta_{j}}^{*}\right) \sin ^{2}\left[2.54 \Delta m_{i j}^{2}(L / E)\right],
\end{aligned}
$$

where $\Delta m_{i j}^{2} \equiv m_{i}^{2}-m_{j}^{2}$ is in $\mathrm{eV}^{2}, L$ is in $\mathrm{km}$, and $E$ is in GeV. In Eq.(1.10) we have used the conventional notation in which, factors of $\hbar$ and $c$ are included to account for the chosen units,

$$
\Delta m_{i j}^{2}(L / 4 E) \approx 1.27 \Delta m_{i j}^{2}\left(\mathrm{eV}^{2}\right) \frac{L(\mathrm{~km})}{E(\mathrm{GeV})}
$$

More detailed treatments of neutrino oscillations [12] that take into account the wave packet nature of the mass eigenstates show that the assumptions made in the derivation shown here are reasonable, but at the same time expose other relevant aspects of the phenomenon.

\section{Two neutrino mixing in vacuum - Average oscillation probability}

In the two neutrino picture where $\alpha=e, \mu$, and $i=1,2$, the probability for a $\nu_{\mu}$ produced with energy $E$ to be detected as a $\nu_{e}$ after traveling a distance $L$ is

$$
P\left(\nu_{\mu} \rightarrow \nu_{e}\right)=\sin ^{2} 2 \theta \sin ^{2}\left(1.27 \Delta m^{2}(L / E)\right)
$$


In a real experiment, the quantity $L / E$ has a certain dispersion. Suppose that $b=1.27(L / E)$ has a Gaussian distribution with standard deviation $\sigma_{b}$ and mean $b_{0}=1.27(L / E)_{0}$. Then the average of the oscillation probability $P(b)$ over all the possible values of $b$ can be calculated as:

$$
\begin{aligned}
\langle P\rangle & =\int_{-\infty}^{\infty} d b P(b) \frac{1}{\sqrt{2 \pi \sigma_{b}^{2}}} e^{-\frac{\left(b-b_{0}\right)^{2}}{2 \sigma_{b}^{2}}} \\
& =\frac{1}{2} \sin ^{2} 2 \theta\left[1-\cos \left(2 b_{0} \Delta m^{2}\right) e^{-2 \sigma_{b}^{2} \Delta m^{2}}\right]
\end{aligned}
$$

Consider the case of an appearance experiment $\left(\nu_{\mu} \rightarrow \nu_{e}\right)$, where measurement of a net excess of electron neutrinos over the expectation is interpreted as two neutrino oscillations. In this situation Eq.(1.13) yields an explicit relation between the unknown parameters $\sin ^{2} 2 \theta$ and $\Delta m^{2}$. The uncertainty on the excess will allow for a region in the parameter space containing all points that are consistent with the measured excess as illustrated in Figure1.1.

In Figure 1.1 (right) the central curve corresponds to the central value of the measured probability interval, and the curves to its left and right are the lower and upper edges of the $1 \sigma$ interval for the parameters.

Note that if the observed excess were consistent with the expectation within the uncertainties, the lower boundary of the interval would be negative and the allowed region would include $\sin ^{2} 2 \theta=0$ as a solution for all values of $\Delta m^{2}$.

\subsection{Direct limits on the neutrino mass}

Direct limits on the neutrino mass have been established by experiments studying the energy spectrum of leptons produced in weak decays. A summary of the most relevant results is given in Table 1.2 . 

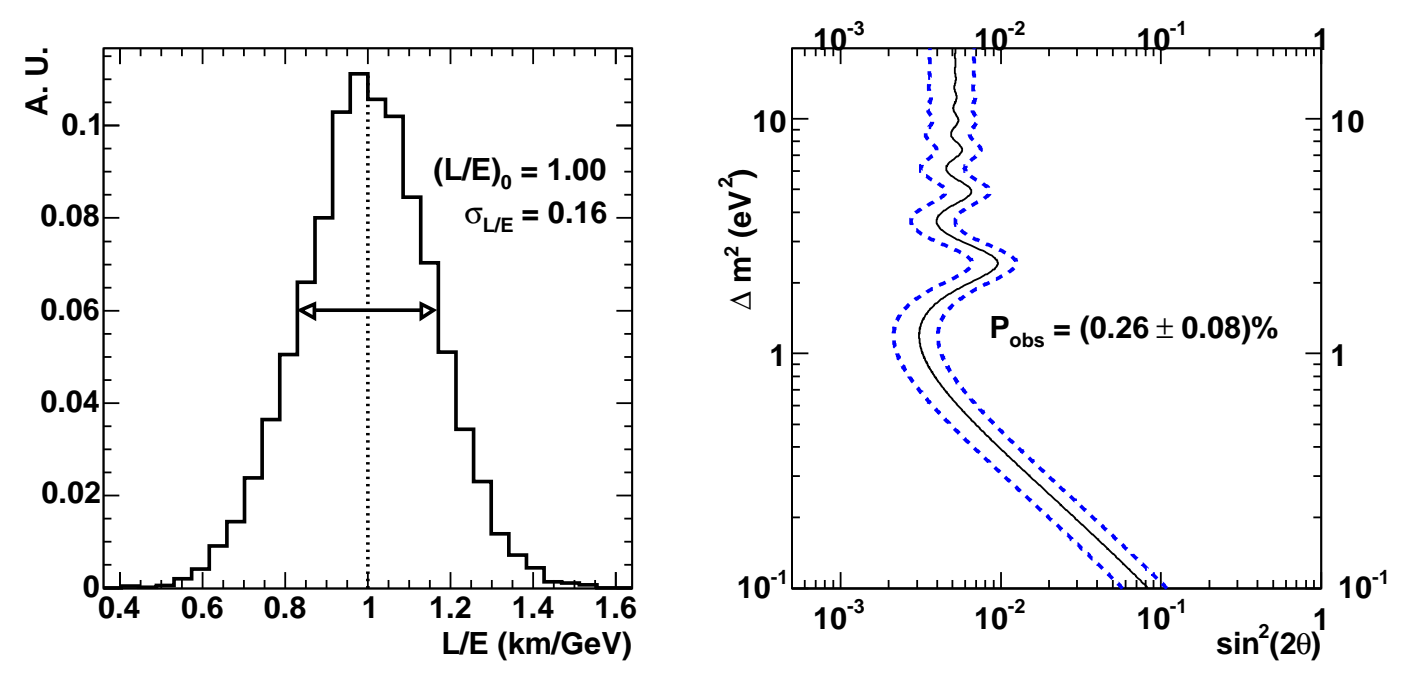

Figure 1.1: On the right, the allowed values of the oscillation parameters obtained from a hypothetical experiment using Eq.(1.13) when observing a net excess of $\nu_{e}$ events of $(0.26 \pm 0.08) \%$ of the expectation with a Gaussian distribution of $L / E$ (left) with mean $(L / E)_{0}$ and standard deviation $\sigma_{L / E}$.

\section{Limits on the electron neutrino mass $\nu_{e}$}

The best absolute limits on the mass of the electron neutrino come from measurements of the $\beta$ decay of tritium $[25,26],{ }^{3} \mathrm{H} \rightarrow{ }^{3} \mathrm{He}+e^{-}+\bar{\nu}_{e}$. Having an energy release $Q=M\left({ }^{3} \mathrm{H}\right)-M\left({ }^{3} \mathrm{He}\right)-m_{e}=18.591 \pm 0.001 \mathrm{keV}[36]$, this decay is particularly sensitive to detect a small neutrino mass of a few $\mathrm{eV} / \mathrm{c}^{2}$. If the electron neutrino produced in the decay is a mixture of the different mass eigenstates $\nu_{e}=\sum_{i} U_{e i} \nu_{i}$, both, mixing and non-zero neutrino masses affect the energy spectrum

$$
\frac{d N}{d E}=R(E)\left(E-E_{0}\right) \sum_{i}\left|U_{e i}\right|^{2}\left[\left(E_{0}-E\right)^{2}-m_{i}\right]^{1 / 2} \Theta\left(E_{0}-E-m_{\nu_{i}}\right)
$$

where $E_{0}=Q+m_{e}$ is the spectrum endpoint for zero neutrino mass, $R(E)$ is a smooth function which does not depend on the neutrino mass [15], and the step function $\Theta\left(E_{0}-E-m_{\nu_{i}}\right)$ guarantees that a neutrino eigenstate of mass $m_{i}$ is created only when there is enough energy available in the decay. A typical experiment will measure 
the integrated count rate above the energy $E_{0}-\delta$, given by $N(\delta)=\bar{R} \int_{E_{0}-\delta}^{E_{0}} \frac{1}{R} \frac{d N}{d E} d E$, since $R(E)$ is a slowly varying function of $E$ whose average over the interval is $\bar{R}$. Performing the integration, the averaged rate near the endpoint is

$$
N(\delta)=\frac{\bar{R}}{3} \sum_{i=1}^{n}\left|U_{e i}\right|^{2}\left(\delta^{2}-m_{i}^{2}\right)^{3 / 2} \Theta\left(\delta-m_{i}\right)
$$

according to which several mass and mixing parameters should be used to fit the experimental data. Tritium $\beta$ decay experiments customarily present their results in terms of a single effective mass $m_{\beta}$, in which case the averaged count rate is simply

$$
N(\delta)=\frac{\bar{R}}{3}\left(\delta^{2}-m_{\beta}^{2}\right)^{3 / 2}
$$

In the limit $\delta^{2}>>m_{i}^{2}$, the effective fitted mass is related to the true masses and mixings by

$$
m_{\beta}^{2} \approx \sum_{i=1}^{n}\left|U_{e i}\right|^{2} m_{i}^{2}
$$

The KATRIN experiment [27] will set an upper limit on $m_{\beta}$ of $0.2 \mathrm{eV}$ at $90 \%$ C.L. if the electron neutrino mass is zero, and will be able to distinguish a neutrino mass of $0.3 \mathrm{eV}(0.35 \mathrm{eV})$ with a significance of $3 \sigma(5 \sigma)$. Results with the expected sensitivity will require three years of data taking, starting in 2010 [28].

\section{Limits on the muon neutrino mass $\nu_{\mu}$}

The strongest limit on the muon neutrino mass has been calculated by K. Assamagan et al. [31] from measurements of the momentum of the muon in pion decay at rest $\pi^{+} \rightarrow \mu^{+}+\nu_{\mu} \quad$, where energy conservation allows one to calculate the squared neutrino mass in terms of the pion mass $m_{\pi}$, the muon mass $m_{\mu}$ and measured momentum $p_{\mu}$ :

$$
m_{\nu_{\mu}}^{2}=m_{\pi}^{2}+m_{\mu}^{2}-2 m_{\pi}\left(m_{\mu}^{2}+p_{\mu}^{2}\right)^{1 / 2}
$$




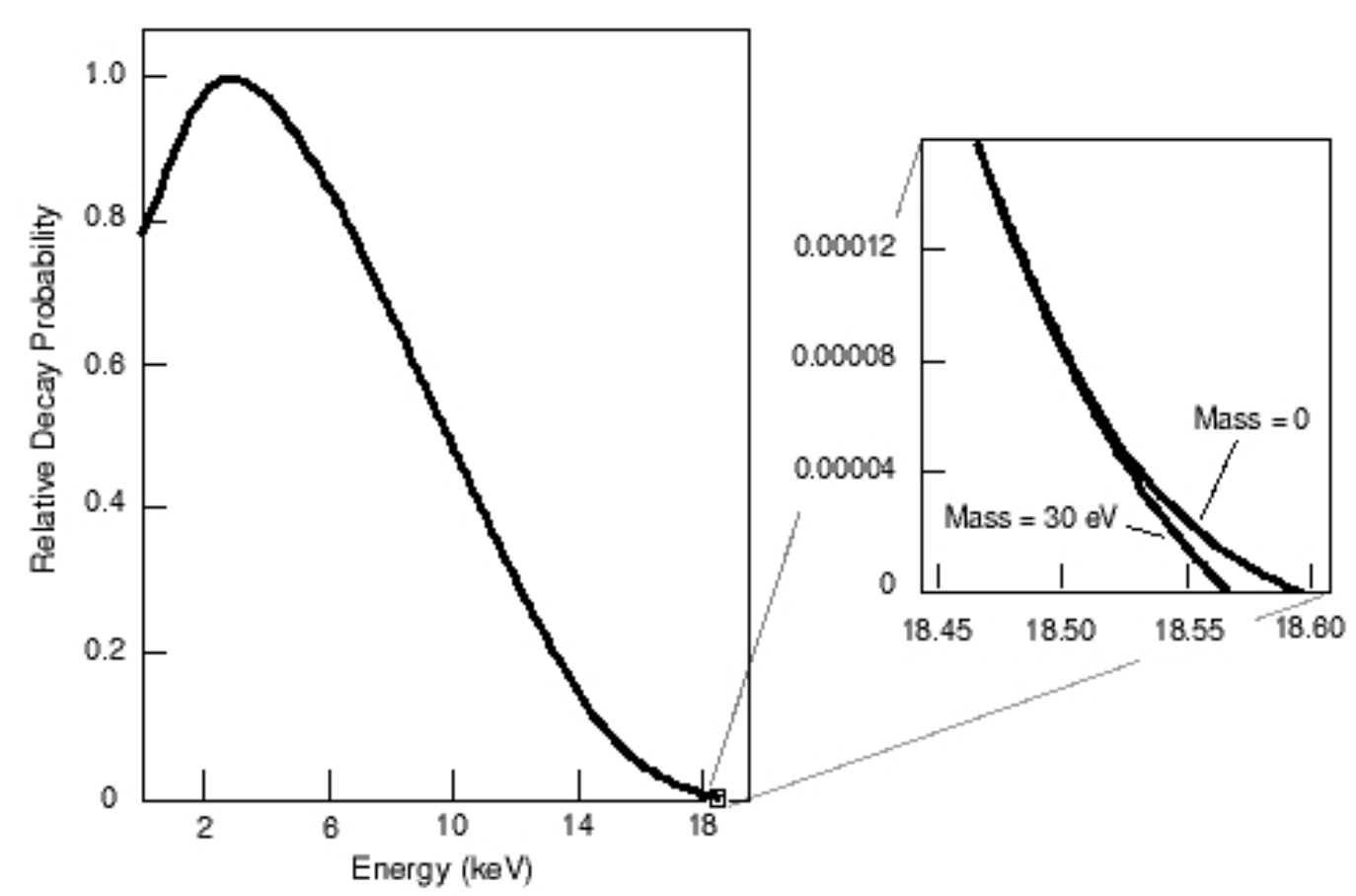

The Beta Decay Spectrum for Molecular Tritium

Figure 1.2: Schematic of the expected effect of a non zero neutrino mass in the tail of the energy spectrum of the electrons emitted in $\beta$ decays like Eq.(1.3). Taken from Ref.[29]

From the result from Assamagan et al. $p_{\mu}=(29.79200 \pm 0.00011) \mathrm{MeV} / c$, and their average for the $\pi^{ \pm}$mass, the authors in Ref.[11] calculate the limit shown in Table 1.2. As in the case of Tritium $\beta$ decays, the value of $m_{\nu_{\mu}}$ extracted by this kinematic measurements is related to the mixings and masses by $m_{\nu_{\mu}}^{2}=\sum_{i=1}^{n}\left|U_{\mu} i\right|^{2} m_{i}^{2}[11]$.

\section{Limits on the tau neutrino mass $\nu_{\tau}$}

The strongest limits have been estimated by R. Barate et al. (ALEPH Collaboration) [33] from kinematic analyses of hadronic decays of the $\tau^{-}$lepton into multi-pion states $\tau^{-} \rightarrow 2 \pi^{-}+\pi^{+}+\nu_{\tau}$ and $\tau^{-} \rightarrow 3 \pi^{-}+2 \pi^{+}\left(+\pi^{0}\right)+\nu_{\tau}$ Combining the limits from the three channels the ALEPH group calculated the limit on $m_{\nu_{\tau}}^{2}=\sum_{i=1}^{n}\left|U_{\tau} i\right|^{2} m_{i}^{2}$ 
shown in Table 1.2.

\begin{tabular}{c|c|c|c} 
& \multicolumn{2}{|c|}{ Table 1.2: Direct limits for neutrino masses. } \\
species & mass limit & decay & reference \\
\hline$\nu_{e}$ & $\leq 3 \mathrm{eV}, 95 \%$ C.L. & ${ }^{3} \mathrm{H} \rightarrow^{3} \mathrm{He}+e^{-}+\bar{\nu}_{e}$ & {$[25,26]$} \\
$\nu_{\mu}$ & $\leq 190 \mathrm{keV}, 90 \%$ C.L. & $\pi^{-} \rightarrow \mu^{-}+\bar{\nu}_{\mu}$ & {$[11]$} \\
$\nu_{\tau}$ & $\leq 18.2 \mathrm{MeV}, 95 \%$ C.L. & $\tau^{-} \rightarrow n \pi+\nu_{\tau}$ & {$[11]$}
\end{tabular}

\section{Neutrino-less double beta decay $\left(\beta \beta_{0 \nu}\right)$}

If neutrinos are Majorana particles and are their own antiparticles certain even-even nuclei can decay according to the reaction $(A, Z) \rightarrow(A, Z+2)+2 e^{-}$, a process that violates lepton number by two units. This process is similar to its lepton number conserving analogue $\left(\beta \beta_{2 \nu}\right)$ where the reaction is $(A, Z) \rightarrow(A, Z+2)+2 e^{-}+2 \bar{\nu}_{e}$, and has been observed in 10 isotopes [17] with half-lives ranging from $10^{19} \mathrm{y}-10^{24} \mathrm{y}$. The rate of $\left(\beta \beta_{0 \nu}\right)$ has been estimated to be given by $\left(T_{1 / 2}^{0 \nu}\right)^{-1}=G_{0 \nu}\left|\mathcal{M}_{0 \nu}\right|^{2}\left\langle m_{\beta \beta}\right\rangle^{2}$ where $G_{0 \nu}$ is a phase factor for the emission of the two electrons, $\mathcal{M}_{0 \nu}$ is a nuclear matrix element for this process, and $\left\langle m_{\beta \beta}\right\rangle^{2}$ is an effective mass parameter given by $\left\langle m_{\beta \beta}\right\rangle^{2}=\left|\sum_{i} U_{e i}^{2} m_{i}\right|$, where cancellations may occur involving possible Dirac or Majorana phases in the matrix $U$. To reach a sensitivity of $\left\langle m_{\beta \beta}\right\rangle^{2} \sim 1 \mathrm{eV}^{2}$, an experiment must be able to observe a half-life $T_{1 / 2}^{0 \nu}$ of about $10^{26} \mathrm{y}-10^{27} \mathrm{y}$ [16]. To date no convincing observation of $\left(\beta \beta_{0 \nu}\right)$ has been made, but a limit on the effective Majorana mass of around $\left\langle m_{\beta \beta}\right\rangle \leq 1 \mathrm{eV}$ can be calculated with existing experimental results [18]. 


\subsection{Experimental observations of neutrino oscilla- tions}

A series of observations of neutrinos from natural sources, as well as from nuclear reactors, gave initial indications of a process not accounted for in the description of neutrinos in the Standard Model. The interpretation of the observed anomalies in terms of massive neutrinos undergoing neutrino oscillations between two states became the standard way to present experimental results. As the experiments became

more precise and laboratory-based searches confirmed the observations from solar and atmospheric neutrinos, the three neutrino picture explanation with a marked mass hierarchy between the states gained force. This picture requires that the sum of two of the mass splittings be equal to the third $\left(\Delta m_{12}^{2}+\Delta m_{23}^{2}=\Delta m_{13}^{2}\right)$, and a mass hierarchy between the neutrinos would allow to use the two independent mass splittings to interpret the different regimes in energy and baseline $(L / E)$ probed by the solar and atmospheric experiments. But this view is inconsistent with the results of the LSND experiment [37, 38, 39, 40], which since the 1990's introduced the possibility of the existence of a fourth neutrino.

It became a usual practice among phenomenologists to exclude the LSND result from global fits to the available data, however this seemed unjustified given its statistical significance, and a new experiment was designed to test the oscillations interpretation of the LSND result. That experiment is MiniBooNE, whose results will be presented in this thesis.

\section{Solar neutrinos}

Measurements of the average rate of solar neutrinos detected with the Chlorine radiochemical experiment at the Homestake mine over more than 20 years of operation, 
yield a ratio of the number of neutrinos observed to those predicted by the Standard Solar Model [42] $(\mathrm{SSM})$ of $R_{C l} / R_{S S M}=0.30 \pm 0.03$, with $R_{C L}=2.56 \pm 0.16 \pm 0.16$ SNU $[43,44]{ }^{6}$. The capture reaction ${ }^{37} \mathrm{Cl}\left(\nu, e^{-}\right)^{37} \mathrm{Ar}$ has an energy threshold of 0.814 $\mathrm{MeV}$, which according to the SSM corresponds to $78 \%$ of the events due to the ${ }^{8} \mathrm{~B}$ flux and $13 \%$ from ${ }^{7}$ Be flux. The average ratio for the radiochemical experiments using a Gallium target SAGE [45] and GALLEX [46] (succeeded by GNO [47]) gave $R_{G a} / R_{S S M}=0.52 \pm 0.03$, with $R_{G a}=68.1 \pm 3.75 \mathrm{SNU}$ [47]. In this case the capture reaction ${ }^{71} \mathrm{Ga}\left(\nu, e^{-}\right)^{71} \mathrm{Ge}$ has a threshold of $0.233 \mathrm{MeV}$, which according to the SSM corresponds to $54 \%$ due to the $p p$ flux, $26 \%$ from ${ }^{7} \mathrm{Be}$, and $11 \%$ from ${ }^{8} \mathrm{~B}$. In addition, the water Cherenkov detectors Kamiokande [48] and Super-Kamiokande [49] in Japan detected ${ }^{8} \mathrm{~B}$ neutrinos in real time through the elastic scattering (ES) reaction $\nu_{a}+e^{-} \rightarrow \nu_{a}+e^{-}(a=e, \mu, \tau)$ with an energy threshold of $5-7 \mathrm{MeV}$. Their results are presented in terms of the ${ }^{8} \mathrm{~B}$ flux yielding a ratio to the SSM prediction of $\Phi_{S K} / \Phi_{S S M}=0.413 \pm 0.014$.

The different flux deficits observed by these experiments $\left(\Phi^{\mathrm{obs}} / \Phi^{\mathrm{SSM}} \sim 0.3-0.6\right)$ suggested an energy dependent effect, which became known as the solar neutrino problem [50], and was resolved by the heavy water SNO detector in the Sudbury mine in Canada. SNO was also sensitive to ${ }^{8} \mathrm{~B}$ neutrinos via the ES reaction but also used the deuterons in $\mathrm{D}_{2} \mathrm{O}$ to observe the $\mathrm{CC}$ reaction $\nu_{e}+d \rightarrow p+p+e^{-}$with an energy threshold of $\sim 5 \mathrm{MeV}$ in its first phase $[51,52,53]$, and the $\mathrm{NC}$ reaction $\nu_{a}+d \rightarrow n+p+e^{-},(a=e, \mu, \tau)$ with an energy threshold of $2.225 \mathrm{MeV}$ in its second phase $[54,55]$. The flavor composition of the solar ${ }^{8} \mathrm{~B}$ flux assuming flavor conversion is related to the fluxes measured by SNO in these three channels:

\footnotetext{
${ }^{6} 1$ Solar Neutrino unit, SNU $\equiv 10^{-36}$ captures/atom/sec
} 


$$
\begin{array}{ll}
\Phi_{S N O}^{C C}=\left(1.68_{-0.06-0.09}^{+0.06+0.08}\right) \times 10^{6} \mathrm{~cm}^{-2} \mathrm{~s}^{-1} & =\Phi_{e} \\
\Phi_{S N O}^{E S}=(2.35 \pm 0.22 \pm 0.15) \times 10^{6} \mathrm{~cm}^{-2} \mathrm{~S}^{-1} & =\Phi_{e}+r \Phi_{\mu, \tau} \\
\Phi_{S N O}^{N C}=\left(4.94 \pm 0.21_{-0.34}^{+0.38}\right) \times 10^{6} \mathrm{~cm}^{-2} \mathrm{~s}^{-1} & =\Phi_{e}+\Phi_{\mu, \tau}
\end{array}
$$

where $r \equiv \sigma_{\mu} / \sigma_{e} \approx 0.15$ is the ratio of the $\nu_{e} e$ and $\nu_{\mu} \mu$ elastic scattering cross sections. The flavor conversion is well described by oscillations of $\nu_{e}$ into $\nu_{\mu}$ or $\nu_{\tau}$ which in a two-neutrino picture correspond to the parameters $\Delta m_{\text {solar }}^{2} \approx 5 \times 10-5$ $\mathrm{eV}^{2}$, and mixing angle $\theta_{\text {solar }} \approx 33^{\circ}$ [41]. This solution is called the large mixing angle (LMA) solution, and arises from the flavor conversion picture described by the Mikheyev-Smirnov-Wolfenstein (MSW) [56] effect in the exponential density profile of the sun.

In August 2007 the Borexino experiment presented its first results [57] on real time observation of ${ }^{7} \mathrm{Be}$ neutrinos, finding it in good agreement with the prediction from the parameters derived from earlier observations.

\section{Atmospheric neutrinos}

Pions and kaons produced in the interaction of cosmic rays with oxygen and nitrogen in the Earth's atmosphere at a mean altitude of $15 \mathrm{~km}$ decay producing $\nu_{e}, \bar{\nu}_{e}, \nu_{\mu}$, and $\bar{\nu}_{\mu}$, with a wide range of energies (from sub-GeV to multi-GeV). Produced via the reaction $\pi \rightarrow \mu \nu_{\mu}$ followed by $\mu \rightarrow e \nu_{\mu} \nu_{e}$, it is expected that the ratio of muon to electron neutrinos from this source be close to $2: 1$. First observations of these neutrinos in experiments in South Africa [58] and India [59], showed disagreement with expectations with low statistical significance, while two calorimeter experiments, Frejus [60] and NUSEX [61] observed no disagreement. Later observations with water Cherenkov detectors Kamiokande [63] and IMB [62] observed a ratio of muon-induced to electron-induced events $\sim 0.6$ smaller than the expected value. These discrepancies 
became known as the atmospheric neutrino anomaly. The zenith angular dependence of the Kamiokande measurements indicated that the deficit was caused mainly by neutrinos coming from below the horizon and traversed the $\sim 10^{4} \mathrm{~km}$ of the Earth's diameter, while those coming from above the horizon traveled only $\sim 15 \mathrm{~km}$. This is further supported by the observations that the deficit grows with the distance traveled by the neutrino from it production point. For multi-GeV energy events Super-Kamiokande found an upward-downward asymmetry in the observed events deviating from the expected value of zero by $\sim 10$ standard deviations $A_{\mu}=\frac{U-D}{U+D}=$ $-0.29 \pm 0,03$, where $\mathrm{U}(\mathrm{D})$ occur in the zenith angle interval $-1<\cos \theta_{z}<-0.2$ $\left(0.2<\cos \theta_{z}<1\right.$.

The high precision and high statistics of the Super-Kamiokande atmospheric data set $[65,66,67]$, together with confirmation from the Soudan2 [69] and MACRO [70] iron calorimeter experiments yield definitive evidence for disappearance of muon neutrinos produced in the atmosphere. The best interpretation of these results is the oscillation of $\nu_{\mu}$ into $\nu_{\tau}$ with oscillation parameters $\Delta m_{\text {atm }}^{2} \approx 2 \times 10^{-3} \mathrm{eV}^{2}$ and mixing angle $\theta_{\text {atm }} \approx 45^{\circ}$ [41]. The explanation in terms of $\nu_{\mu} \rightarrow \nu_{e}$ oscillations is excluded at high C.L. because the $\nu_{e}$ events agree well with the expectation and would have produced a deficit in the CHOOZ reactor experiment that was not observed. Oscillations into sterile neutrinos $\nu_{\mu} \rightarrow \nu_{s}$ are also ruled out for atmospheric neutrinos because this implies a suppression of the NC signal that was not observed [71]. The interpretation in terms of no $\nu_{\tau}$ appearance is found to he disfavored at $2.4 \sigma[72]$ according to a recent analysis of the effects of this hypothesis.

\section{Reactor Neutrinos}

Nuclear reactors are sources of exclusively $\bar{\nu}_{e}$ with energies of only a few MeV. With energies this low a $\bar{\nu}_{e}$ that oscillates into a different flavor would be impossible to 
detect with conventional detectors. There is not enough energy to produce a $\mu$ or a $\tau$, which is what a conventional detector needs to tell that the transition took place. Therefore, reactor experiments look typically for a $\bar{\nu}_{e}$ disappearance signal.

The experiments Gosgen [73] in Switzerland ( $L \sim 38-65$ m,) Krasnoyarsk [74] in Russia $(L \sim 57-231 \mathrm{~m})$, Bugey [75] $(L \sim 50-94 \mathrm{~m})$ and CHOOZ [76] in France $(L \sim 1 \mathrm{~km})$, and Palo Verde [77] in Arizona, U.S. $(L \sim 750-890 \mathrm{~m})$, found no evidence of neutrino oscillations placing exclusion limits on the region of the parameter space above $\sin ^{2} 2 \theta \gtrsim 10^{-1}$ and $\Delta m^{2} \gtrsim 10^{-3} \mathrm{eV}^{2}$. The exclusion region from $\mathrm{CHOOZ}$ extends to $\Delta m^{2}$ values which are relevant to the interpretation of atmospheric neutrino data, and have important implications in global fits invoking the three-neutrino picture.

The ongoing KamLAND experiment [78] observes $\bar{\nu}_{e}$ from several nuclear reactors around the Kamioka mine in Japan, which are located at an average distance ranging from 150 to $210 \mathrm{~km}$ from a liquid scintillator detector. At these longer baselines the experiment is sensitive to neutrino oscillations with $\Delta m^{2} \gtrsim 10^{-5} \mathrm{eV}^{2}$, similar to that effecting solar neutrinos. Expressed in terms of a ratio of the number of observed events to the expectation for no oscillations for $E_{\bar{\nu}_{e}}>3.4 \mathrm{MeV}$, the first KamLAND result is $R_{\text {KamLAND }}=0.611 \pm 0.094$ [79], and the energy dependence of the observed deficit is well described by neutrino oscillations [80, 81]. This result demonstrates that electron anti-neutrinos oscillate with parameters consistent with those for electron neutrinos from the Sun, which is the expected behavior if CPT is a symmetry of leptonic processes. A combined analysis of the solar and KamLAND data yields oscillation parameters $\Delta_{\text {solar }+ \text { KamLAND }} \approx 8 \times 10^{-5} \mathrm{eV}^{2}$, and $\theta_{\text {solar }+ \text { KamLAND }} \approx 34^{\circ}[41]$. This solution is called the large mixing angle (LMA) solution to the solar neutrino problem, in contrast to other solutions that had smaller mixing angles that were possible before the KamLAND experiment. With the introduction of KamLAND 
data into global analyses of solar neutrino data the LMA solution unambiguously emerged as the favoured solution to the solar neutrino problem.

\section{Accelerator neutrinos with long baselines}

Laboratory made neutrino beams [82] are derived from the decays of charged $\pi$ and $K$ mesons, which are in turn produced from proton beams striking thick nuclear targets.

$$
\begin{aligned}
p+X_{\text {target }} \rightarrow & \pi^{ \pm}+Y \\
& \pi^{ \pm} \rightarrow \mu^{ \pm}+\nu_{\mu}\left(\bar{\nu}_{\mu}\right) \\
& \mu^{ \pm} \rightarrow e^{ \pm}+\nu_{e}\left(\bar{\nu}_{e}\right)+\bar{\nu}_{\mu}\left(\nu_{\mu}\right)
\end{aligned}
$$

The characteristics of the neutrino beam obtained depend on the precise selection and manipulation of the mesons produced at the target.

The first long baseline accelerator neutrino experiment was K2K [83] which produced neutrinos at the KEK laboratory from a $12 \mathrm{GeV} / c$ momentum proton beam impacting a fixed aluminum target, and the neutrinos traveled a distance $L \sim 235$ $\mathrm{km}$ to the Super-Kamiokande detector. The second of its kind is the ongoing MINOS experiment [84] with a baseline $L \sim 730 \mathrm{~km}$ between the beam, produced from 120 $\mathrm{GeV} / \mathrm{c}$ momentum protons from the Fermilab Main Injector impacting a graphite target, and an iron calorimeter detector in Soudan, MI in the US.

The results from K2K $[85,86]$ and MINOS [87, 88] show in each case, an energy dependent deficit consistent with oscillations of $\nu_{\mu}$ over distances of order several $\sim 10^{2} \mathrm{~km}$ with parameters that are consistent with those describing atmospheric neutrino oscillations.

An emulsion detector with lead as passive target called OPERA [89], at the Gran Sasso Underground Laboratory (LNGS) in Italy is located at a distance of 730 $\mathrm{km}$ from its neutrino source at CERN (CNGS beam), and was designed to test the 
$\nu_{\mu} \rightarrow \nu_{\tau}$ hypothesis for the atmospheric neutrino oscillations through the appearance of $\nu_{\tau}$ in a beam of $\nu_{\mu}$. The experiment reported the observation of the first neutrinos from the first run of the CNGS beam in August 2006 [90].

\section{Accelerator neutrinos with short baselines}

Accelerator-based neutrino oscillations experiments in which the distance from the production area to the detectors is of order a few hundred meters are referred to as short baseline (SBL) experiments. So far there has only been one reported observation of short baseline neutrino oscillations (experimental results summarized in Table 1.4), the LSND experiment, whose result is described in the next section.

\section{LSND}

The LSND experiment [40] (Liquid Scintillator Neutrino Detector) was performed at the Los Alamos Neutron Science Center (LANSCE) at Los Alamos National Laboratory between 1993 and 1998 to search for neutrino oscillations of the type $\bar{\nu}_{\mu} \rightarrow \bar{\nu}_{e}$. The neutrino sources of LSND include the pion decay modes $\pi^{+} \rightarrow \mu^{+} \nu_{\mu}$ and $\pi^{+} \rightarrow e^{+} \nu_{e}$, occurring both at rest (DAR) as well as in flight (DIF). The decays $\pi^{-} \rightarrow \mu^{-} \bar{\nu}_{\mu}$, and $\pi^{-} \rightarrow e^{-} \bar{\nu}_{e}$ occur only in flight, since $\pi^{-}$are readily absorbed on nuclei when they stop. Also relevant are the muon decay modes $\mu^{+} \rightarrow e^{+} \nu_{e} \bar{\nu}_{\mu}$ and $\mu^{-} \rightarrow e^{-} \bar{\nu}_{e} \nu_{\mu}$. Nearly all the $\mu^{+}$stop before decaying and produce a normal Michel energy spectrum for $\nu_{e}$ and $\bar{\nu}_{\mu}$, while the already small fraction of $\mu^{-}$produced from the $\pi^{-}$DIF are either absorbed in a nucleus or decay in orbit around one. The $\bar{\nu}_{e}$ flux is calculated to be only $\sim 8 \times 10^{-4}$ as large as the $\bar{\nu}_{\mu}$ flux in the energy range of $20<E_{\nu}<52.8 \mathrm{MeV}$. The potential oscillation signal $\bar{\nu}_{\mu} \rightarrow \bar{\nu}_{e}$ derives from the $\bar{\nu}_{\mu}$ flux from the isotropic $\mu^{+}$DAR. The $\bar{\nu}_{e}$ flux from $\mu^{-}$DAR is a background to this signal whose energy spectrum is similar to that of the $\nu_{e}$ from $\mu^{+}$used to verify the 


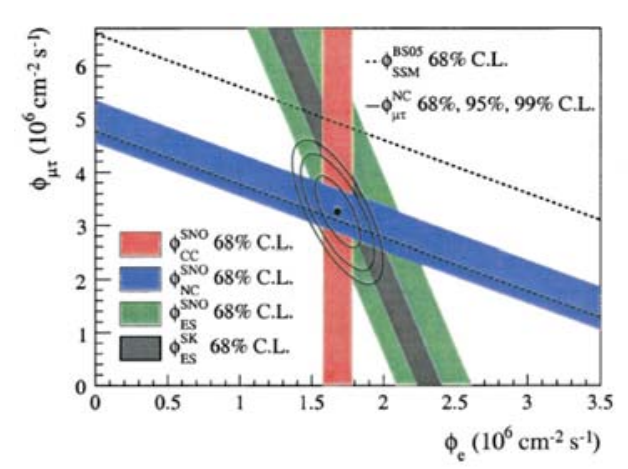

(a) SNO, from $[55]$

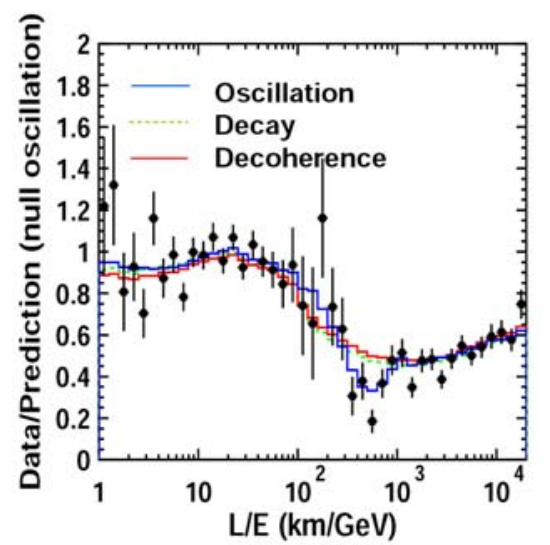

(c) Super-Kamiokande, from [68]

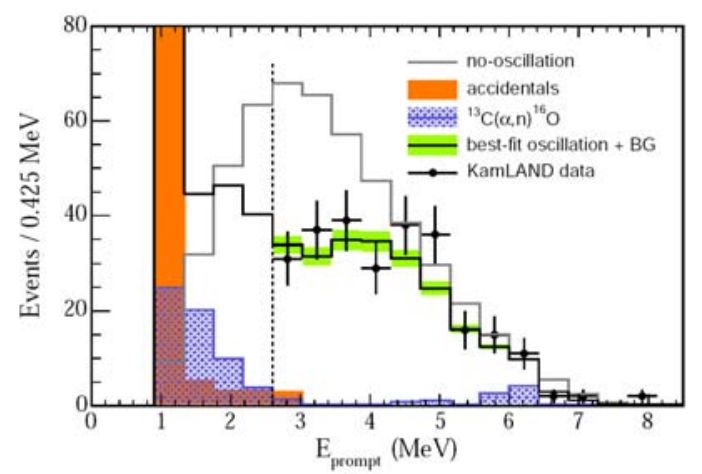

(b) KamLAND, from [80]

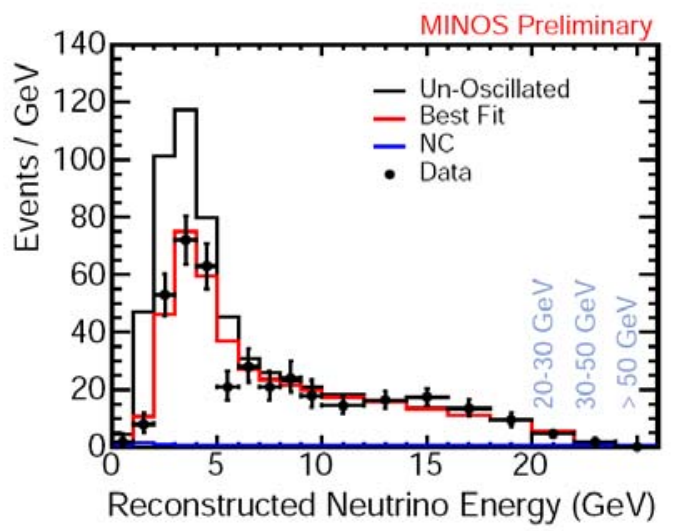

(d) MINOS, from [88]

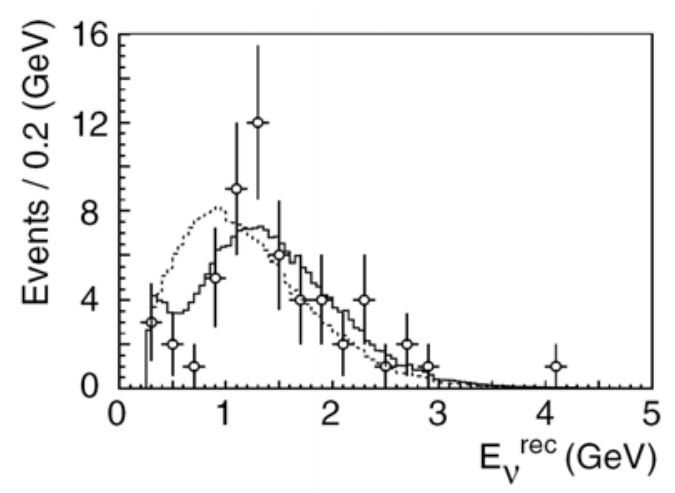

(e) $\mathrm{K} 2 \mathrm{~K}$, from $[86]$

Figure 1.3: A partial collection of results from experiments described in the text observing (a) solar, (b) reactor, (c) atmospheric, and (d-e) accelerator neutrino oscillations. In (e) the (dashed) solid histogram is the expected (un)oscillated spectrum. These measurements have firmly established that neutrinos have non-zero masses and mixings. 
Table 1.3: 90\% C.L. limit on the oscillation probability from searches at short baseline experiments with negative results[41].

\begin{tabular}{|c|c|c|c|c|c|}
\hline \hline Experiment & Laboratory & Channel & $\operatorname{limit}(90 \%)$ & $\Delta m_{\min }^{2}\left(e V^{2}\right)$ & Ref. \\
\hline CDHSW & CERN & $\nu_{\mu} \longrightarrow \nu_{e}$ & $P_{\mu \mu}>0.95$ & 0.25 & {$[91]$} \\
E776 & BNL & $\nu_{\mu} \longrightarrow \nu_{e}$ & $P_{e \mu}<1.5 \times 10^{-3}$ & 0.075 & {$[92]$} \\
E734 & BNL & $\nu_{\mu} \longrightarrow \nu_{e}$ & $P_{e \mu}<1.6 \times 10^{-3}$ & 0.4 & {$[93]$} \\
KARMEN2 & Rutherford & $\bar{\nu}_{\mu} \longrightarrow \bar{\nu}_{e}$ & $P_{e \mu}<6.5 \times 10^{-4}$ & 0.05 & {$[94]$} \\
E531 & FNAL & $\nu_{\mu} \longrightarrow \nu_{\tau}$ & $P_{\mu \tau}<2.5 \times 10^{-3}$ & 0.9 & {$[95]$} \\
CCFR & FNAL & $\nu_{\mu} \longrightarrow \nu_{\tau}$ & $P_{\mu \tau}<4 \times 10^{-3}$ & 1.6 & {$[96]$} \\
& & $\nu_{e} \longrightarrow \nu_{\tau}$ & $P_{e \tau}<0.1$ & 20.0 & {$[97]$} \\
& & $\nu_{\mu} \longrightarrow \nu_{e}$ & $P_{\mu e}<9 \times 10^{-4}$ & 1.6 & {$[98]$} \\
Chorus & CERN & $\nu_{\mu} \longrightarrow \nu_{\tau}$ & $P_{\mu \tau}<3.4 \times 10^{-4}$ & 0.6 & {$[99]$} \\
& & $\nu_{e} \longrightarrow \nu_{\tau}$ & $P_{e \tau}<2.6 \times 10^{-2}$ & 7.5 & {$[99]$} \\
Nomad & CERN & $\nu_{\mu} \longrightarrow \nu_{\tau}$ & $P_{\mu \tau}<1.7 \times 10^{-4}$ & 0.7 & {$[100]$} \\
& & $\nu_{e} \longrightarrow \nu_{\tau}$ & $P_{e \tau}<7.5 \times 10^{-3}$ & 5.9 & {$[100]$} \\
& & $\nu_{\mu} \longrightarrow \nu_{e}$ & $P_{\mu e}<6 \times 10^{-4}$ & 0.4 & {$[100]$} \\
\hline \hline
\end{tabular}


background estimates. A cylindrical tank $8.3 \mathrm{~m}$ long and $5.7 \mathrm{~m}$ in diameter lined with 1220 PMT's was centered $30 \mathrm{~m}$ away from the neutrino source. The tank was filled with 167 t of liquid scintillator and was used to look for an excess of events consistent with the reaction $\bar{\nu}_{e} p \rightarrow e^{+} n$, over the expected number from the $\mu^{-}$DAR background sources. The second most relevant background are events consistent with the reaction $\bar{\nu}_{\mu} p \rightarrow \mu^{+} n$ from $\pi^{-}$DIF around the source. These reactions were identified through the correlation of a prompt signal from the $e^{+}\left(\mu^{+}\right)$, and a $2.2 \mathrm{MeV} \gamma$ from the capture of the $n$. Accidental photons are distinguished by means of a correlatedto-accidental likelihood ratio $R_{\gamma}$, which depends on number of PMT's associated with the $\gamma$, the reconstructed distance from the $\gamma$ to the $e^{+}$, and the time difference between the two (the capture time of thermal neutrons in mineral oil is $186 \mu \mathrm{s}$, while accidental photons occur uniformly in time). A $\chi^{2}$ fit to the $R_{\gamma}$ distribution of events with $20<E_{e}<60 \mathrm{MeV}$ and satisfying cuts on the time and angular dependence of the emitted light is shown in Fig.1.4(a). The fit gives a (beam-on)-(beam-off) excess of $117.9 \pm 22.4$ events with a correlated neutron. After subtracting the neutrino background from $\mu^{-}$DAR (19.5 \pm 3.9 events), and from $\pi^{-}$DIF (10.5 \pm 4.6 events) [102], LSND obtains a total excess of $87.9 \pm 22.4 \pm 6.0$ events, corresponding to an oscillation probability of $(0.264 \pm 0.067 \pm 0.045) \%$, where the first error is statistical and the second is the systematic error mainly due to uncertainties in the neutrino flux $(7 \%)$ and the $e^{+}$and $\gamma$ efficiencies (7\%). Fig.1.5(a) shows the allowed regions in the oscillation parameters space $\left(\sin ^{2} 2 \theta, \Delta m^{2}\right)$ obtained from a fit to the entire data sample including the effect of a possible $\nu_{\mu} \rightarrow \nu_{e}$ signal from the $\nu_{\mu}$ flux.

\section{The KARMEN experiment}

The KArlsruhe Rutherford Medium Energy Neutrino experiment [94] shares many similarities with LSND, but it observed no evidence for neutrino oscillations. KAR- 


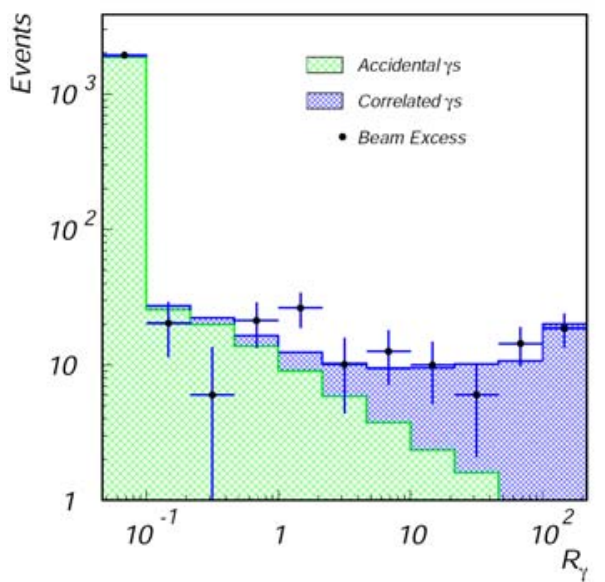

(a) $R_{\gamma}$ for events satisfying the $\bar{\nu}_{\mu} \rightarrow$

$\bar{\nu}_{e}$ search criteria

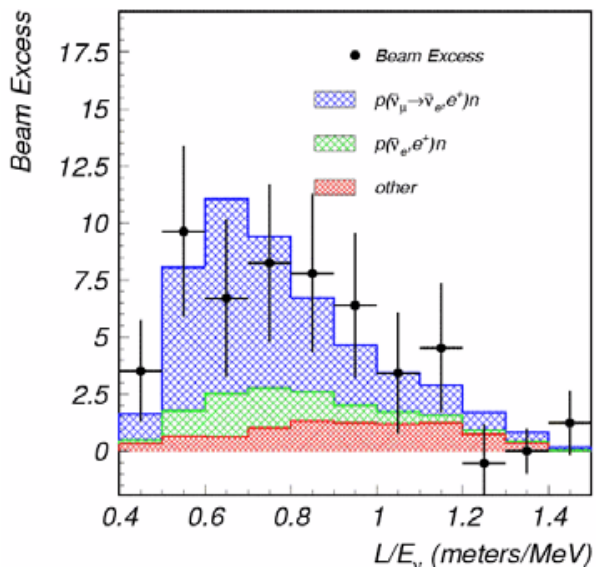

(b) $L / E$ distribution of LSND excess events with $R_{\gamma}>10$

Figure 1.4: In (a) the $R_{\gamma}$ distribution of LSND events satisfying the $\bar{\nu}_{\mu} \rightarrow \bar{\nu}_{e}$ search criteria described in the text. In (b) the $L / E$ distribution of events in the signal region of the $R_{\gamma}$ distribution.

MEN looked for $\bar{\nu}_{\mu} \rightarrow \bar{\nu}_{e}$ oscillations from a DAR source with a negligible DIF component using a smaller (mass of $56 \mathrm{t}$ ) but similar detector, and the same reaction channel $\left(\bar{\nu}_{e} p \rightarrow e^{+} n\right)$ as LSND. KARMEN observed 15 events passing their selection criteria, which showed good agreement in time, energy and position distributions with the expected $15.8 \pm 0.5$ events.

LSND and KARMEN collaborators studied the compatibility of the observations of the two experiments and concluded [101] that the differences in baseline, flux and analysis techniques made their results compatible at the $64 \%$ level. Assuming statistical compatibility a joint analysis yields the allowed regions shown in Fig.1.5(b).

This joint analysis used LSND data from the Decay At Rest (DAR) channel only by requiring $R_{\gamma}>0$. As a result, the joint KARMEN-LSND allowed region at $90 \%$ is shifted to slightly larger values of $\sin ^{2} 2 \theta$ with respect to the LSND-only allowed region. 


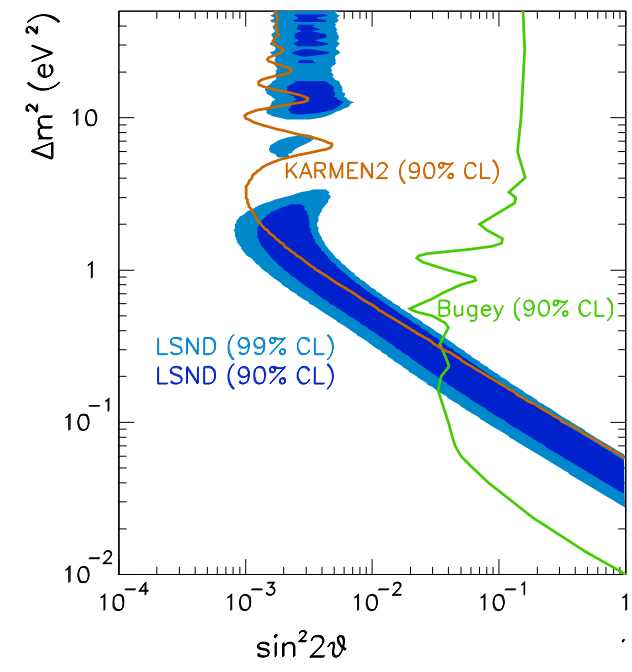

(a)

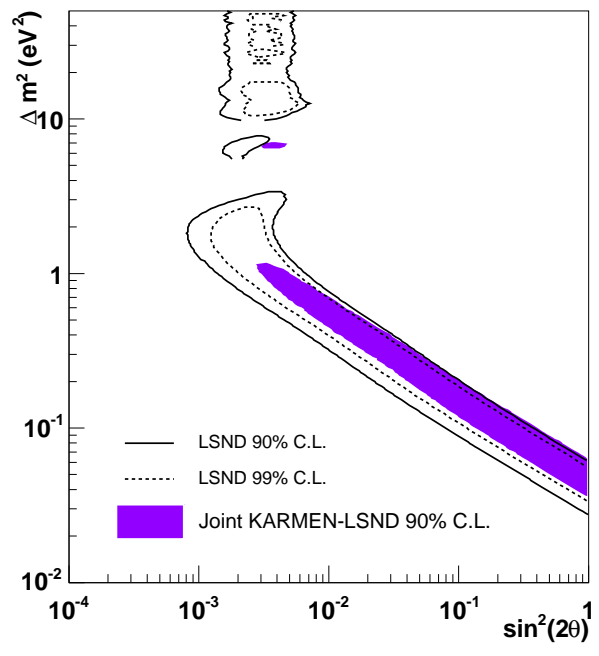

(b)

Figure 1.5: (a) Allowed oscillation parameter regions for the entire LSND DAR and DIF data with $20<E_{e}<200 \mathrm{MeV}$. Inner (outer) regions are 90\% (99\%) C.L. The KARMEN and Bugey limits are shown for comparison; (b) The allowed regions at 90\% C.L. for the joint analysis of LSND and KARMEN [101] with LSND DAR data only $\left(R_{\gamma}>0\right)$ which causes the shift with respect to the LSND regions in (a), overlaid for comparison. 


\section{Chapter 2}

\section{The MiniBooNE Experiment}

An intense neutrino beam is produced from the collision of protons with a beryllium (Be) target located inside a magnetic focusing horn. The positive mesons produced by the interactions of the protons with the target material are focused by the magnetic field of the horn and allowed to decay. Neutrinos from the decay of these mesons travel a distance of approximately $541 \mathrm{~m}$ before reaching the MiniBooNE detector. Negative mesons are also produced, but they are defocused by the magnetic field and their contribution to the neutrino flux through the detector is greatly suppressed.

\subsection{The proton beam of the Booster Neutrino line}

The Fermilab Booster accelerator delivers protons with $8 \mathrm{GeV}$ kinetic energy (8.9 $\mathrm{GeV} / c$ momentum) to a beryllium target in $1.6 \mu$ s pulses with $\sim 5 \times 10^{12}$ protons per pulse at $15 \mathrm{~Hz}$ with a maximum average rate of $\sim 5 \mathrm{~Hz}$. Figure 2.1 shows how the proton beam is extracted into the $8 \mathrm{GeV}$ neutrino line before being injected into the Main Injector. The beam intensity, position and profile are constantly monitored with toroids, beam position monitors (BPM), and multiwire arrays respectively. To 
prevent the beam from moving too far from its nominal position and deposit energy in the horn material, a donut shaped collimator and beam loss monitor are installed by the upstream end of the target to trip the beam if necessary. A program called Autotune [119] corrects the beam position and angle in real time and minimizes any misalignment.

\subsection{Target, Focusing Horn, and decay pipe}

The MiniBooNE target assembly, see Figure 2.2 (left), consists of 7 cylindrical Be slugs $\sim 10 \mathrm{~cm}$ in length and $1 \mathrm{~cm}$ in diameter, two concentric Be tubes, and an aluminum manifold at the upstream end. Three radial cooling fins are placed symmetrically around each cylindrical slug (not shown in the figure). Air enters through channels in the manifold and flows in the space between the fins and the outer beryllium tube to lower the target temperature. The Be tubes and slugs are placed inside the inner conductor of a magnetic focusing horn, with the target axis aligned with the proton beam impinging on its upstream face with a typical beam spot size of $\sim 1 \mathrm{~mm}$ across. The $65 \mathrm{~cm}$ of the target length are equivalent to 1.7 proton interaction lengths. The target assembly is kept in electrical contact with the horn inner conductor through stainless steel bellows to prevent damages arising from electrical arching between the two assemblies.

The purpose of the focusing horn ${ }^{1}$ is to create a magnetic field with the correct characteristics to produce the desired secondary beam of mesons. The inner and outer conductors of the MiniBooNE horn are shown in Figure 2.2 (right). The outer conductor is a cylinder with inner surface radius of $30.0 \mathrm{~cm}$, while the inner conductor has an outer surface radius that varies from $2.2 \mathrm{~cm}$ to $3.9 \mathrm{~cm}$ depending on the

\footnotetext{
${ }^{1}$ Designed by Bartoszek Engineering [197].
} 


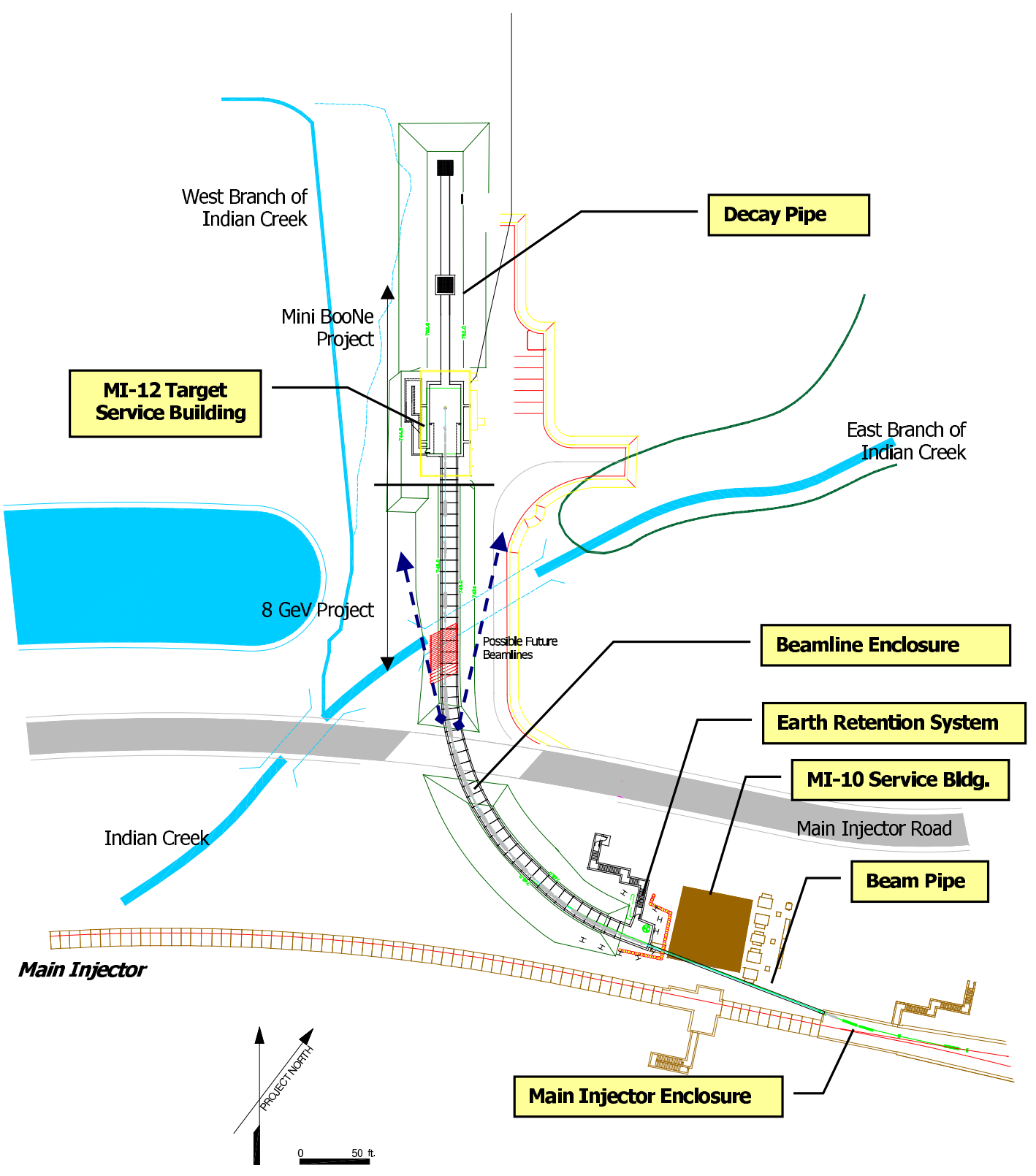

Figure 2.1: Schematic of the $8 \mathrm{GeV}$ beamline with extraction facility [116]. Taken from [116]. 

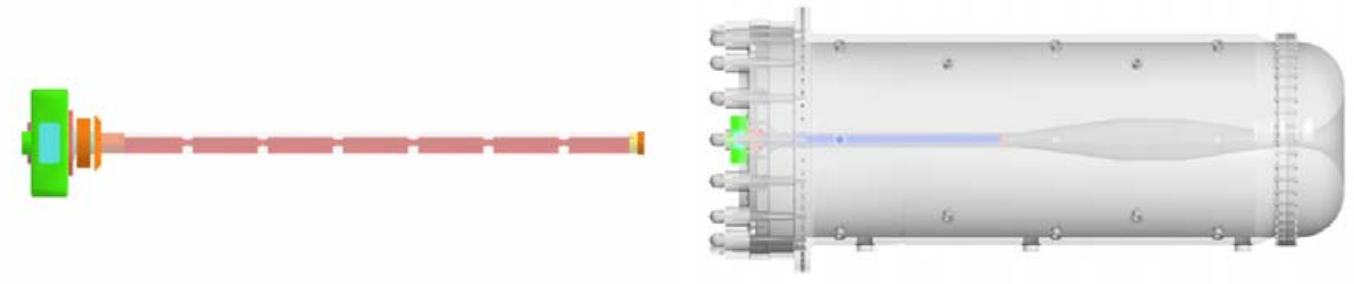

Figure 2.2: Three-Dimensional rendering of the target beryllium slugs (left) and the MiniBooNE focusing horn with the target in place (right). Images Provided by L. Bartoszek [197].

longitudinal position along the horn axis. The two conductors are connected by an end cap that transports a current of 170,000 Amperes injected at the upstream end of the inner conductor to the outer conductor. The magnitude of the current and the shape of the inner conductor were optimized using a GEANT [198] simulation to maximize the $\nu_{\mu}$ flux between 0.5 and $1 \mathrm{GeV}$ at the detector, while minimizing the flux above $1 \mathrm{GeV}$. The current flow produces a toroidal magnetic field in the region between the conductors well described by a $1 / r$ dependence. Measurements of the magnetic field inside the horn have been made [105] showing that the deviation of the magnetic field from the idealized case of two infinite conducting cylinders due to edge effects is smaller than $5 \%$. In the region outside the two conductors the magnetic field is negligibly small.

The decay pipe is a $50 \mathrm{~m}$ tubular enclosure $1 \mathrm{~m}$ in diameter, filled with air at normal atmospheric pressure that provides the necessary room for high momentum mesons produced in the target to decay. At the end of the decay pipe is a concrete beam absorber that stops the residual mesons that did not decay within the pipe and also stops the part of the proton beam that did not interact with the target, and any remaining hadronic component of the beam.

When the horn is operated with positive polarity the secondary meson beam is 
composed almost entirely of $\pi^{+}$with energies around $\sim 2 \mathrm{GeV}$, which upon decaying through the dominant mode $\pi^{+} \rightarrow \nu_{\mu} \mu^{+}$(99.988\% branching ratio, BR), will produce an almost pure beam of $\nu_{\mu}$. Most of the $\mu^{+}$from $\pi^{+}$decay will reach the $50 \mathrm{~m}$ absorber and capture on a nucleus, however any $\mu^{+}$decaying in flight through $\mu^{+} \rightarrow e^{+} \bar{\nu}_{\mu} \nu_{e}$ will contribute to the $\nu_{e}$ contamination of the beam. $K^{+}$and $K^{0}$ will also be produced at the target and produce $\nu_{e}$ 's through the decays $K^{+} \rightarrow \pi^{0} e^{+} \nu_{e}(4.87 \% \mathrm{BR})$, and $K^{0} \rightarrow \pi^{ \pm} e^{\mp} \nu_{e}(38.8 \% \mathrm{BR})$. We refer to these sources of $\nu_{e}$ as intrinsic.

Roughly $10 \mathrm{~m}$ upstream of the absorber a $17 \mathrm{~m}$ long pipe is inserted into the decay pipe at an angle of $\sim 7^{\circ}$, leading to the Little Muon Counter (LMC). The LMC is designed to observe muons from the off axis decay of kaons moving along the decay pipe. This device provides a good check of the number of $K$ mesons produced at the target and hence on the modeling of the intrinsic $\nu_{e}$ contamination from $K^{+}$ decays. More details on the LMC can be found elsewhere [118].

\subsection{Neutrino beam and detector}

The detector is a spherical tank $6.1 \mathrm{~m}$ in radius filled with $\sim 950,000$ lt of ultra pure mineral oil (density of $0.845 \mathrm{~g} / \mathrm{cm}^{3}$ ). The detector volume is divided by an optical barrier into an inner region $5.5 \mathrm{~m}$ in radius and an outer region with $6.1 \mathrm{~m}$ radius. The inner region observed by 1280 inward-facing 8 -inch photomultiplier tubes (PMTs), while the outer region is instrumented with 240 PMTs facing tangentially to the tank wall as can be seen in Figure 2.3(b).

The PMTs detect the light produced as charged particles traverse the oil, providing 10\% active photocathode coverage of the inner tank wall. The detector is particularly suited to detect the Cherenkov radiation from relativistic particles moving with speeds larger than the speed of light in the oil, although the PMTs are also 


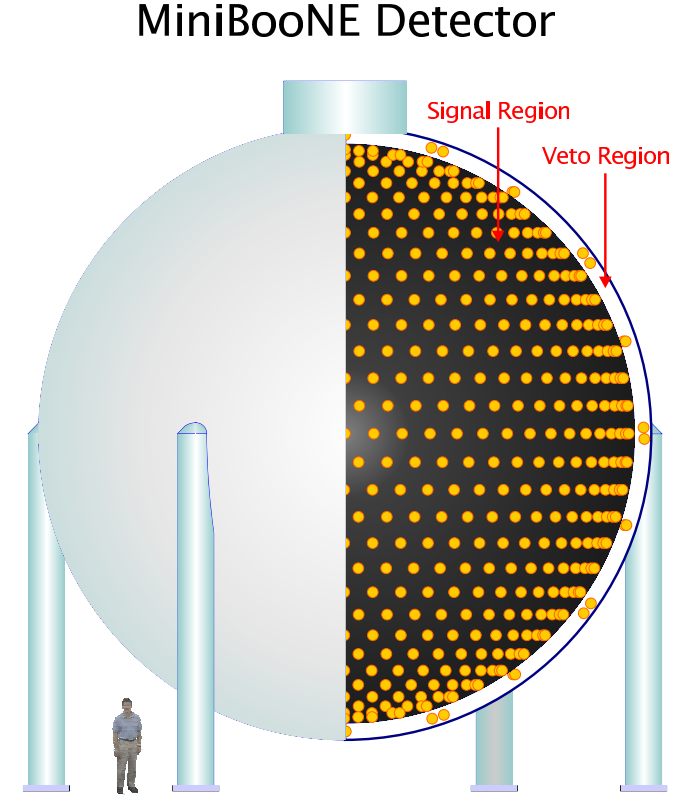

(a)

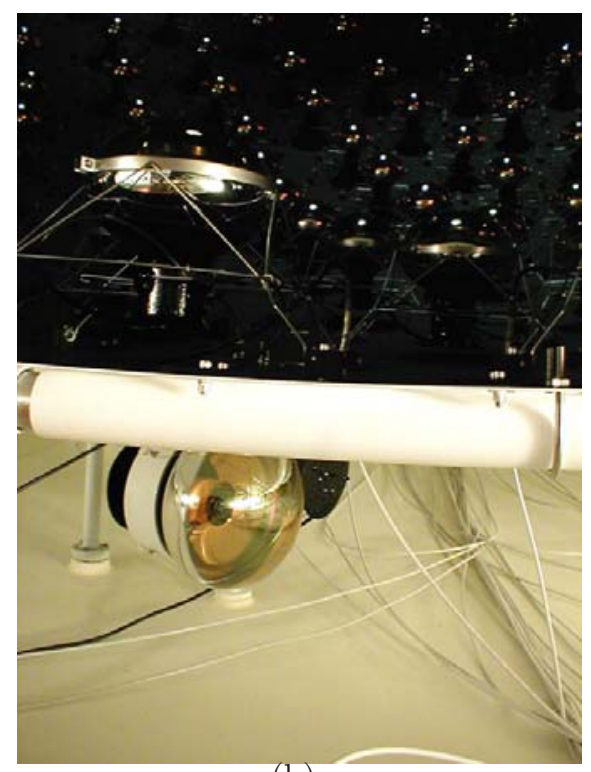

(b)

Figure 2.3: (a) The MiniBooNE detector; (b) Photograph showing the arrangement of PMTs inside the tank (black area) and the veto region (white area).

sensitive to isotropic light emitted through natural scintillation processes in the oil. The outer region, called veto is used to detect charged particles entering the tank from the outside (e.g. cosmic ray muons), and provides a rejection of $99.9 \%$ of cosmic background events. The tank is located under $3 \mathrm{~m}$ of overburden (see Figure 2.3), which keeps the rate of cosmic ray muons through the tank below $10 \mathrm{kHz}$.

The experiment uses 1197 PMTs used previously in the LSND experiment (Hamamatsu [199] R1408) plus 323 new tubes (Hamamatsu R5912) that were required due to the discontinuation of the production of the R1408 model. Both types of tubes are 8" in diameter fabricated from low radioactivity glass, a major difference being that the R1408 has a 9-stage dynode chain while the the R5912 has a 10-stage dynode chain. The smaller number if dynodes in the R1408 causes them to produce a significantly lower anode pulse $(\sim 1.5 \mathrm{mV})$ for a single photoelectron $(\mathrm{PE})$ event at the operation voltage, while the R5912 produces a typical $25 \mathrm{mV}$ anode output at 
the same operating voltage. A preamplification factor of $\times 19$ was added to the high voltage distribution system for every R1408 tube to reduce the difference in output anode voltages. The tubes are operated with the anode at a positive high voltage and the cathode at ground, as required in their specifications [200]. The operating voltage of each tube is set by a series of trim resistors on the inputs to the preamplifiers, allowing for the use of fixed voltage supplies to power all the tubes.

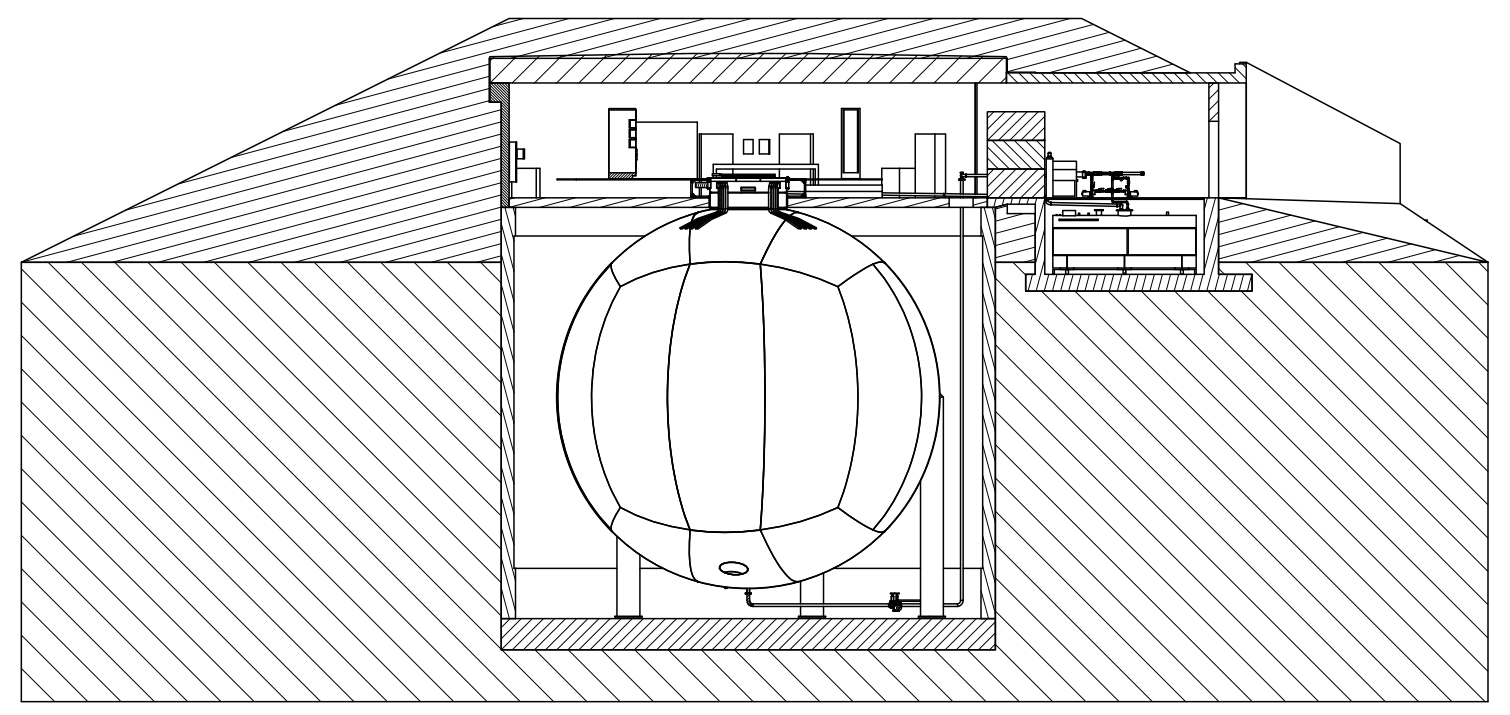

Figure 2.4: Elevation view of the detector inside its containment plant, showing the oil overflow tank.

The number of neutrinos passing through the detector volume is calculated with a GEANT4 based simulation containing the details of the meson production, the geometry of the horn, the decay pipe, and the detector. The detector is located at a distance of $541 \mathrm{~m}$ from the target. The contributions from $\nu_{\mu}$ and $\nu_{e}$ are shown in Figure 2.5. 


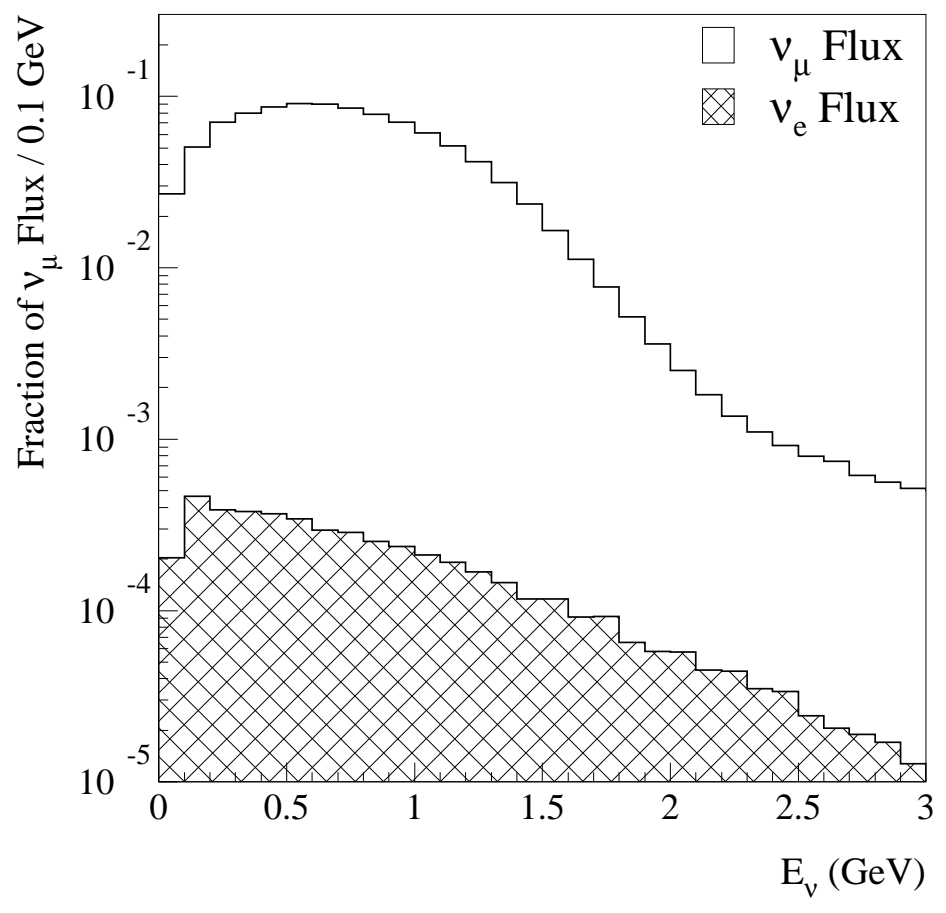

Figure 2.5: Predicted $\nu_{\mu}$ and $\nu_{e}$ flux distributions as a function of neutrino energy in MiniBooNE.

\subsection{Data Acquisition and Calibration systems}

\section{Electronics and Data Acquisition}

The fast PMT anode pulses are converted into two new slower analog voltages, one proportional to the charge of the pulse $(\mathrm{Vq})$ and a second one proportional to the time at which the pulse occurs (Vt). Groups of 8 PMTs are read out by one card that performs this charge/time conversion $\left(\mathrm{QT}\right.$ board $\left.^{2}\right)$. The QT boards are arranged in groups of 16 into 12 crates controlled by a VME-based monoboard computer, giving a total of $16 \times 8 \times 12=1536$ available channels for the 1520 PMTs. Within each QT board, the Vq and Vt signals (illustrated in Figure 2.6) are digitized by two

\footnotetext{
${ }^{2}$ Originally designed for LSND by Vern Sandberg, Los Alamos National Laboratory.
} 
separate ADC's at $10 \mathrm{MHz}$ and stored in circular buffer memories which overwrite every $2048 \times 100 \mathrm{~ns}=204.8 \mu \mathrm{s}$.

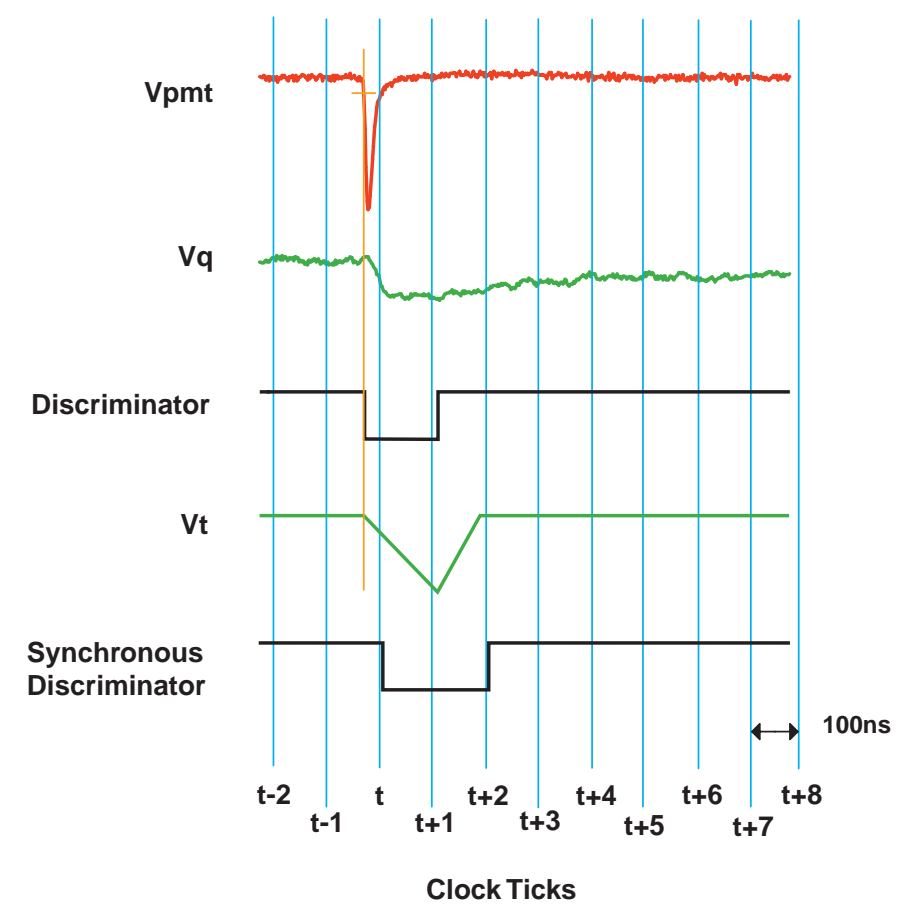

Figure 2.6: PMT charge and time signals. Vpmt and Vq in the figure are the anode pulse and its integral respectively (both analog signals). When the anode pulse crosses a preset threshold the "Discriminator" digital pulse is started, along with the analog Vt time ramp. Vq and Vt are digitized by FADCs every 100 ns (clock ticks). Taken from [117].

In Figure 2.6 the signal Vq is the voltage across an integrating capacitor whose feeding voltage is the PMT anode signal $\mathrm{V}_{\mathrm{pmt}}$. If the anode signal is large enough to fire an on-board discriminator (set to $\sim 0.25$ photoelectrons), the voltage signal Vt begins to ramp up linearly from the PMT baseline until two clock ticks have elapsed, time at which it is reset to the PMT baseline voltage before the next clock tick. Both, Vq and Vt as described above are digitized at every 100 ns clock tick.

The signals for the ADC conversion are provided by a GPS referenced $10 \mathrm{MHz}$ clock. At every $100 \mathrm{~ns}$ clock tick the digitized Vq and Vt are stored at a memory 
location in the circular buffer that corresponds to the number of clock ticks in the $10 \mathrm{MHz}$ clock. When a trigger condition is met a trigger broadcast module sends out a set of timestamp addresses (TSAs) to all the buffer memories, and the data in these addresses is transfered from the buffers to a set of First-In-First-Out (FIFO) memories that hold it until it can be read and processed by the VME-based mono board computer. If the trigger decision takes longer than $204.8 \mu$ s to request data from a particular set of TSAs, the circular buffer overwrites them and the data is lost $^{3}$. A latency filter is applied to all analyzed data to reject events in which this occurs [106].

Each QT board also contains a PMT sum card which counts the number of channels that caused the discriminator to fire in the last two clock ticks (200 ns). This information is sent to the trigger crate, which takes care of the "sum of sums" for the main tank and the veto separately to give an overall number of PMTs in each of these two detector regions.

The mono-board computers process the time and charge information in $\mathrm{Vq}$ and Vt for each tube and reduce it to four Vq values (the charge quad) and four Vt values (the time quad) which are sent to the main acquisition computer. In each case one element of the quad occurs before the discriminator fires, and three occur after. These are equivalent to simple charge values and the time they occured with subnanosecond resolution. The detector data stream consists of the following information for each PMT hit:

1. The PMT channel number

2. The clock tick, counted from the start of the event, that preceeds the firing of the discriminator.

\footnotetext{
${ }^{3}$ The fraction of beam events lost due to latency is typically $\sim 0.1 \%$
} 
3. The four recorded Vq values in ADC counts (the charge quad)

4. The four recorded Vt values in ADC counts (the time quad)

When a PMT hit saturates the ADC range (by having more than $\sim 20$ photoelectrons), the DAQ writes out additional charge quads until Vq is small enough to fit on the ADC range. The charge and time quads are used to extract the time at which each hit occured relative to the other hits in a given event, and the number of photoelectrons that correspond to each hit. The time and charge of a hit are calculated as follows

$$
\begin{aligned}
t & =t_{\text {raw }}+(100 \mathrm{~ns}) \times N_{\text {tick }}+\Delta t_{\text {offset }}+\Delta t_{\text {slew }}\left(Q_{\text {raw }}\right) \\
q & =Q_{\text {raw }} / \text { Gain }
\end{aligned}
$$

where $t_{\text {raw }}$ is the time of the hit relative to the preceeding clock tick, which is obtained from the slope of the ramping Vt signal and its intersection with the baseline. $N_{\text {tick }}$ is the number of the clock tick that precedes the firing of the discriminator, and is the coarse measure of the time of the hit. The term $\Delta t_{\text {offset }}$ is a channel-dependent calibration constant that removes time differences originating from things such as differing cable lengths and differing dynode structures between PMT's ${ }^{4}$. The term $\Delta t_{\text {slew }}\left(Q_{\text {raw }}\right)$ is a charge-dependent time-slewing correction read from a look-up table ${ }^{5}$. The PMT gains and time slewing corrections are determined with the laser calibration system described next.

\footnotetext{
${ }^{4}$ The set of new PMTs has 10 dynodes, while the set of old PMTs from LSND has 9.

5 The raw charge $Q_{\text {raw }}$ is proportional to the PMT anode charge and is determined solely from the information in the charge quad.
} 


\section{The LASER calibration system}

Light from a pulsed diode laser is sent via optical fibers to four light dispersing flasks distributed in various positions throughout the interior of the MiniBooNE detector. The purpose of these devices is to provide a controlled source of photons that can be used to monitor the time offsets and gains of individual PMTs. The flasks are $10 \mathrm{~cm}$ in diameter and are filled with Ludox ${ }^{\circledR}[108]$ as dispersive medium ${ }^{6}$.

Table 2.1: Laser flask positions in beam coordinates $(z$ along beam, $y$ upward, $x$ such that system is right-handed, origin at tank center).

\begin{tabular}{c|ccc}
\hline \hline Flask \# & $\mathrm{x}(\mathrm{cm})$ & $\mathrm{y}(\mathrm{cm})$ & $\mathrm{z}(\mathrm{cm})$ \\
\hline 1 & -0.08 & 1.52 & -4.35 \\
2 & -28.18 & 78.81 & 0.98 \\
3 & 83.34 & 2.67 & 203.69 \\
4 & -97.11 & -165.92 & 96.23 \\
\hline \hline
\end{tabular}

The diode laser is pulsed asynchronous to the proton beam at $3.3 \mathrm{~Hz}$ continuously during normal data taking. A switch box is used to send the $\leq 100$ ns width pulses to each of the four flasks. The position of the flasks inside the tank is shown in Figure 2.8 .

The time slewing corrections and PMT gains of Eq.(2.1) are determined separately for the R1408 and R5912 PMTs. The time slewing corrections are obtained from runs with a variety of light intensities, while the PMT gains are calculated by fitting the single photoelectron (PE) peak for each PMT in low intenstiy runs [111]. Low intensity laser runs are also used to determine the PMTs quantum efficiencies

\footnotetext{
${ }^{6}$ Ludox ${ }^{\circledR}$ colloidal silicas are aqueous colloidal dispersions of very small silica particles, opalescent as white milky white liquids.
} 
and charge-likelihood tables, both key to the reconstruction algorithms (see Section $3.4)$.

These calibrations are regularly stored in look-up tables approximately every four days, and are primarily performed using the central flask (flask \#1). Data from the other three flasks and from a Michel electron sample are used to cross-check the calibrations. The corrected time distribution for both types of PMTs is shown in Figure 2.7.

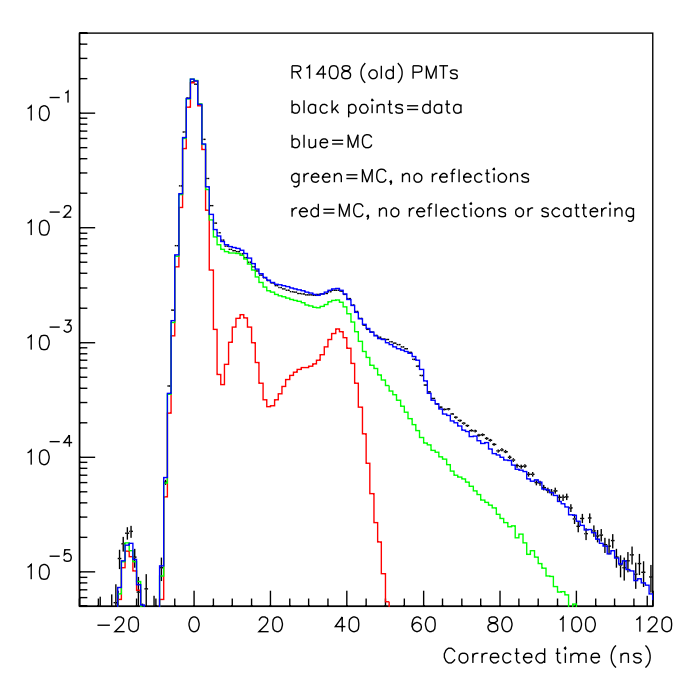

(a) R1408 (old PMTs)

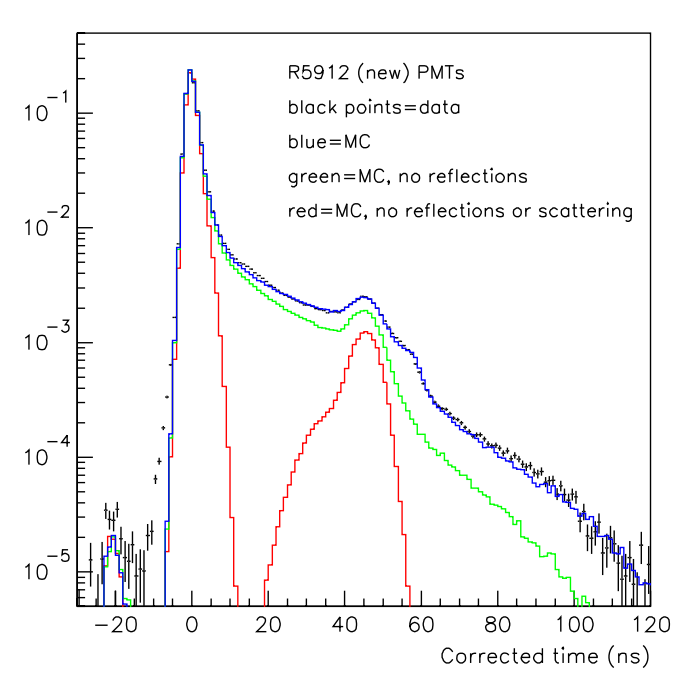

(b) R5912 (new PMTs)

Figure 2.7: Corrected time distribution PMT hits for $397 \mathrm{~nm}$ light from laser flask 1. Data are shown in black points and simulation in blue. The green histogram shows the simulation without reflections. The red histogram has no reflections and no scattering (Rayleigh and Raman), showing only the simulated PMT response. Variations in transit time of electron cascades down the PMT dynode stack are the leading contribution to the PMT timing resolution. See Ref.[180] for a detailed description of the various features.

The speed of light in the oil can also be measured from low intensity laser data. Using the light from flask 4 gives a value of the speed of light of $c_{n}=18.7 \pm 0.5$ 
$\mathrm{cm} / \mathrm{ns}$ [111], which is consistent with the expected value of $19.3 \mathrm{~cm} / \mathrm{hs}$ used in the reconstruction algorithms ${ }^{7}$.

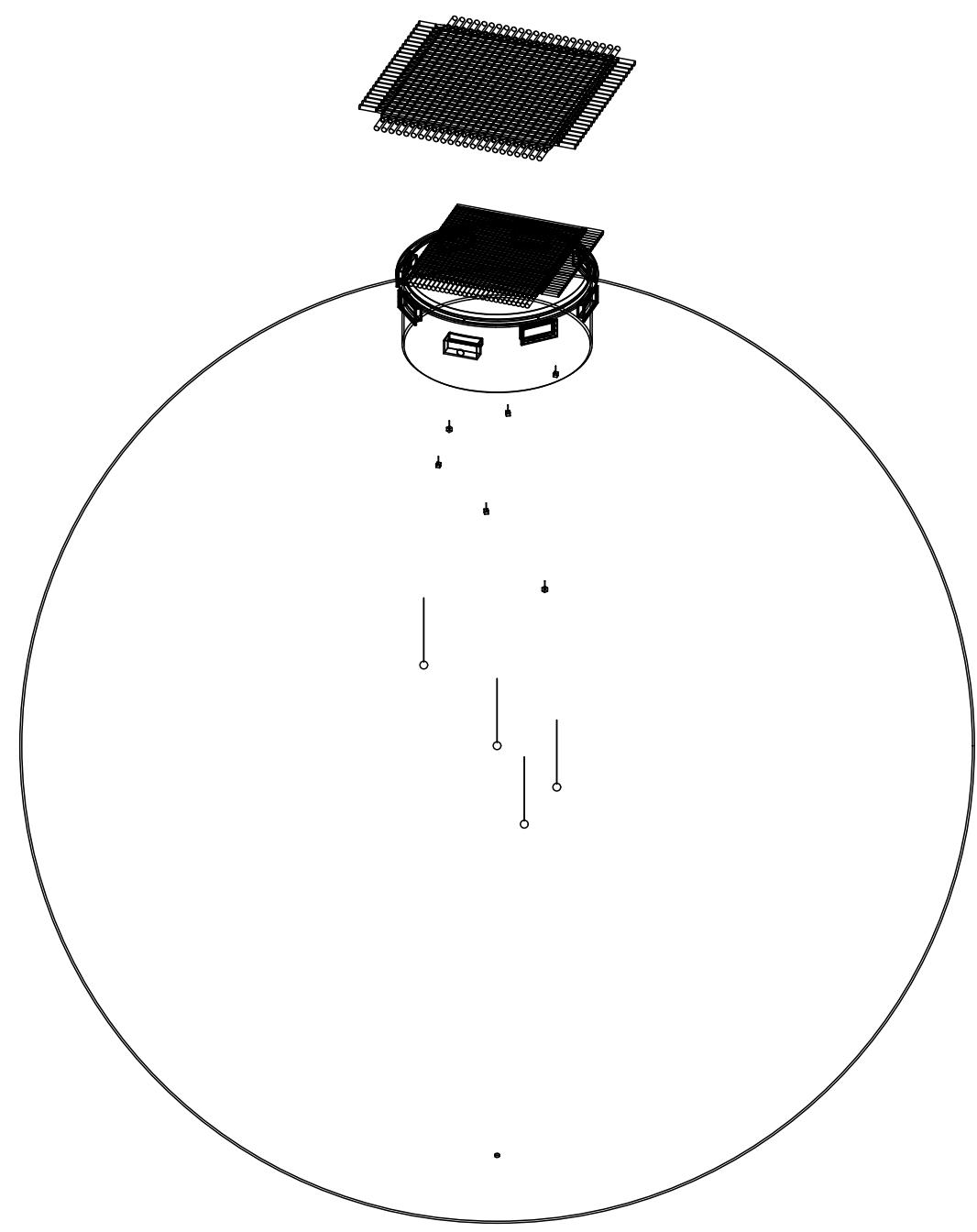

Figure 2.8: MiniBooNE calibration systems. The figure shows the two planes of the Muon Tracker hodoscope system, the 7 scintillation cubes (black cubes) and the 4 laser flasks (white circles) in their positions inside the tank. Taken from [117].

\footnotetext{
${ }^{7}$ The exact value of the speed of light in the oil is not critical to the performance reconstruction algorithms.
} 


\section{The Muon Tracker and scintillation cubes}

A tracking hodoscope [109] is used to measure the entry point and direction of cosmic ray muons that penetrate the MiniBooNE tank. The hodoscope measures the $(x, y)$ coordinates of an entering cosmic ray muon at two fixed heights $z_{b}$, and $z_{t}$ above the tank $\left(z_{t}-z_{b}=150 \mathrm{~cm}\right)$. At each of the two heights, one plane of scintillator strips is used to determine the $x$ coordinate and a second plane, with strips running perpendicular to the first one, is used to determine the $y$ coordinate. This system allows for a reconstruction of muon tracks with an angular resolution of $\sim 6^{\circ}$.

Seven optically isolated scintillation cubes are distributed at various positions inside the detector and are connected by an optical fiber to a 1 inch PMT for readout. They are used together with the Muon Tracker hodoscope to obtain a precise determination of the energy of stopping muons from their stopping range [110]. The cosmic ray calibration system (muon hodoscope and cubes) are shown in they surveyed positions in Figure 2.8.

For a downward going muon that enters the tank and stops in one of the scintillation cubes, a three point fit to a straight line is performed using the two points from the Muon Tracker and the position of the cube. Then the fitted line is intersected with the sphere defined by the optical barrier of the tank to obtain the entry point of the muon (the solution with $y_{\text {int }}>y_{\text {cube }}$ is chosen). The muon range is the distance from the entry point to the cube. The position of the various cubes ranges from 15 $\mathrm{cm}$ to $400 \mathrm{~cm}$ from the tank surface, corresponding to energies of stopping muons from $20 \mathrm{MeV}$ to $800 \mathrm{MeV}$, which are determined from tables of ionization energy loss based in the Bethe-Bloch formula with various corrections:

$$
-\frac{d E}{d x}=2 \pi a_{0}^{2} m_{e} c^{2} n_{e}\left(\frac{Z}{\beta}\right)^{2}\left[\ln \left(\frac{2 m_{e} c^{2} \gamma^{2} \beta^{2} T_{\max }}{I^{2}}\right)-2 \beta^{2}-\delta-2 \frac{C}{Z}\right]
$$

where $a_{0}$ is the Bohr radius, $m_{e}$ is the electron mass, $c$ is the speed of light, $I$ is the 
mean ionization potential of the medium through which the particle travels, $\beta$ and $\gamma$ are the usual relativistic factors, $T_{\max }$ is the maximum kinetic energy that can be imparted to a free electron in a single collision, $\delta$ is the density effect correction to the energy loss, and $C$ is a shell correction [112].

A comparison of the energy obtained from the range of muon events crossing through the Muon Tracked hodoscope and stopping in a scintillation cube, and the energy determined from the light in the $\operatorname{tank}^{8}$ is shown in Figure 2.9.

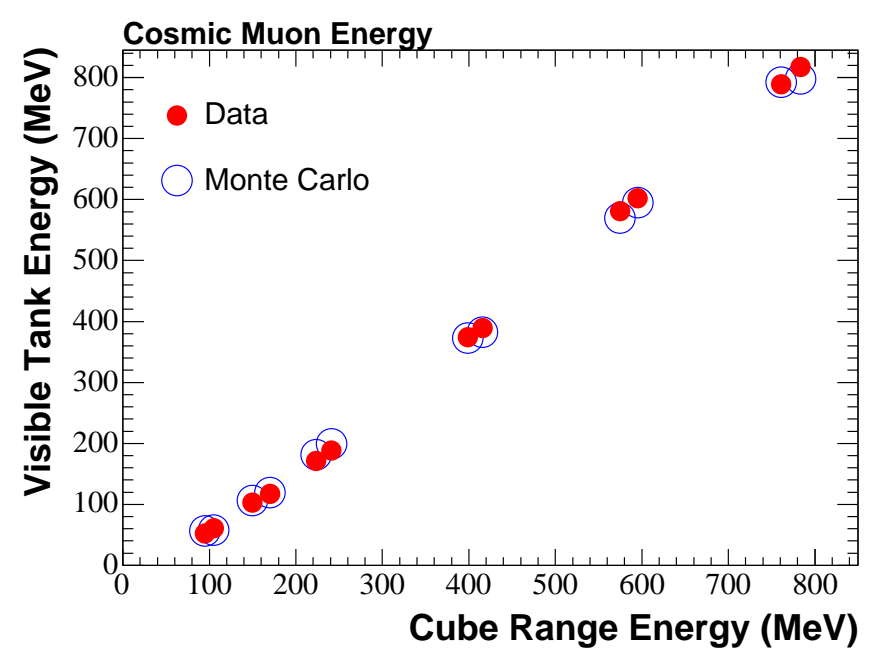

Figure 2.9: Energy determined from light in the tank (vertical axis) versus energy determined with the cosmic muon tracker and cubes system (horizontal axis) for a sample of muons passing through the tracking hodoscope and stopping in the scintillation cubes. The visible tank energy is reconstructed with the S-Fitter algorithm described in Section 3.4. For more details on this calibration see [110].

The sample of stopping muons for the calibration of the energy reconstruction algorithms is assembled by requiring a delayed coincidence between a muon that stops in a cube and its subsequent decay electron. This sample provides an absolute energy calibration with a resolution of $\pm 3 \%$ in the relevant energy range for these events.

\footnotetext{
${ }^{8}$ From the S-Fitter algorithm described in Section 3.4.
} 


\section{Michel electron energy calibration}

The energy spectrum of electrons from the decay of stopped muons (Michel electrons) provides another absolute way to calibrate the energy of events in the tank. The observed energy distribution of these electrons, shown in Figure 2.10, provides a measurement of the energy resolution at the endpoint of the Michel electron spectrum of $52.8 \mathrm{MeV}$.

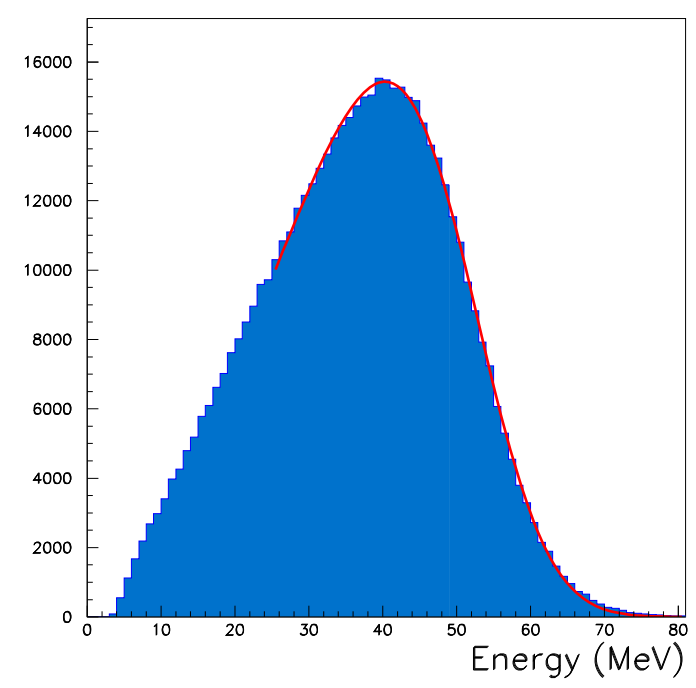

Figure 2.10: Observed Michel electron energy spectrum (histogram) and the best fit (solid curve) obtained by smearing the theoretical energy spectrum with a Gaussian with width proportional to $\sqrt{E}$. The energy resolution determined from the fit is $14.8 \%$ at $52.8 \mathrm{MeV}$.

The Michel electron sample is also used to tune the reconstruction algorithms [114] described in Section 3.4, and to check the PMT calibration constants determined from laser runs. This sample is also used to study the optical properties of the oil and plays an important role in determining the systematic uncertainties in the optical model of the detector. 
Table 2.2: Internal trigger bits and their thresholds.

\begin{tabular}{ccccc|cc}
\hline \hline \multicolumn{3}{c|}{ Sum over Main Tank PMT Hits } & \multicolumn{2}{c}{ Sum over Veto PMT Hits } \\
DET1 & DET2 & DET3 & DET4 & DET5 & VETO1 & VETO2 \\
\hline$\geq 10$ & $\geq 24$ & $\geq 200$ & $\geq 100$ & $\geq 60$ & $\geq 6$ & $\geq 4$ \\
\hline \hline
\end{tabular}

\section{Trigger conditions}

Both internal and external triggers can cause data to be written to disk. Internal triggers are based on information from the main and veto sum cards in the trigger crate. Seven internal trigger bit types are asserted if simple requirements on the number of PMT signals in the main and veto regions are met. The names and settings of the internal trigger bits are shown in Table 2.2. Four input connections on the front of the trigger crate (labelled E1 through E4) handle the various external triggers.

Beam on target trigger (E1):

Timing information in the accelerator complex is transmitted to all Fermilab areas through optical fiber running along communication ducts. The Booster broadcasts two timing signals relevant to the MiniBooNE beam trigger called " $\$ 1 \mathrm{D} "$ and " $\$ 1 \mathrm{~F} "$. A \$1D signal indicates that the Booster is preparing to send a beam pulse to MiniBooNE, and occurs $25 \mathrm{~ms}$ before the $1 \mathrm{~F}$ signal. The $1 \mathrm{~F}$ signal precedes the beam extraction kick by $320 \mu \mathrm{s}$. A coincidence between two TTL signals (TTL1 and TTL2) that take into account the time delays and transport times of the $\$ 1 \mathrm{D}$ and $\$ 1 \mathrm{~F}$ signals, as well as the beam time of flight to the target is used to set the E1 bit. As a result, the information from TSAs in a $19.2 \mu$ s wide window starting $4.5 \mu$ s before the neutrinos arrive at the detector are retrieved and stored to disk. A holdoff of $20 \mu \mathrm{s}$ is enforced after a beam trigger before the trigger can register new activity. 
Strobe Trigger (E2):

This trigger bit can be set by four different triggering events: strobe, debuncher, follower, and NuMI. The different triggers sharing the E2 trigger bit are distinguished from each other by the width of a TTL pulse $\left(\Delta t_{\text {pulse }}\right)$ produced when the particular triggering condition is met. For the strobe trigger $\left(\Delta t_{\text {pulse }}=350 \mathrm{~ns}\right)$, a pulse generator set to a frequency of $2.01 \mathrm{~Hz}$ is used to retrieve tank activity in a $19.2 \mu$ s window ${ }^{9}$. A signal from the accelerator complex indicating that beam is about to be sent to the anti-proton source target ${ }^{10}$ is used for the debuncher trigger $\left(\Delta t_{\text {pulse }}=550 \mathrm{~ns}\right)$ [107]. The Follower trigger $\left(\Delta t_{\text {pulse }}=150 \mathrm{~ns}\right)$ is aimed to look for neutron capture events after a beam or strobe event, and is delayed $20 \mu$ s from a beam or strobe trigger. The NuMI trigger $\left(\Delta t_{\text {pulse }}=750 \mathrm{~ns}\right)$ is designed to observe events from the nearby NuMI neutrino beam at Fermilab. For a description of this trigger see Appendix G.

\section{Calibration trigger (E3):}

Similar to the E2 trigger bit, the E3 bit shares four types of triggering events all related to calibration data: the CALIB_CUBE $\left(\Delta t_{\text {pulse }}=150 \mathrm{~ns}\right)$, triggered by activity in the scintillation cubes, CALIB_LASER $\left(\Delta t_{\text {pulse }}=450 \mathrm{~ns}\right)$, triggered by pulses of the calibration laser, CALIB_TRACKER $\left(\Delta t_{\text {pulse }}=650 \mathrm{~ns}\right)$, triggered by a 4 plane coincidence in the Muon Tracker system, or a councidence between the cubes and the tracker, and CALIB_BEAM $\left(\Delta t_{\text {pulse }}=850 \mathrm{~ns}\right)$, triggered by a laser pulse asynchronous with a Booster pulse.

Hardware OR (E4):

This is a NIM hardware OR of the E1, E2, E3 external bits, and the internal bits. An assertion of the E4 bit enforces the storage of the time and current state (on or

\footnotetext{
${ }^{9}$ The strobe trigger is used to study the detector activity when no beam is present.

${ }^{10}$ Accelerator tclk signal $\$ 81$.
} 
off) of ALL internal and external trigger bits.

\subsection{Neutrino events in the tank}

The DAQ system opens a $19.2 \mu$ s window for every proton spill delivered by the Booster beam to the experiment starting $4.6 \mu$ s before the begining of the spill. A distribution of the time of a collection of PMT hits for a fraction of a spill typically displays clusters in time that are produced by individual particle tracks in the detector. These clusters are called sub-events.

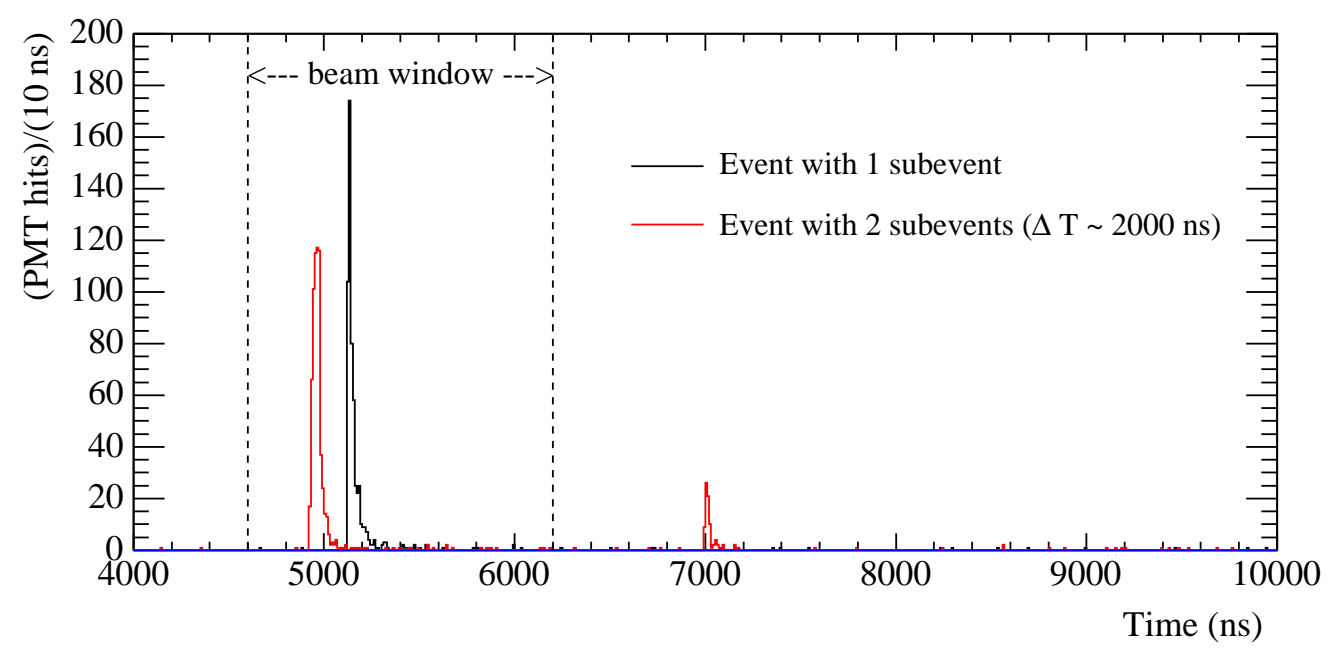

Figure 2.11: Time distribution of PMT hits for an event with 1 sub-event (black) and an event with two sub-events (red). The dotted vertical lines delimit the beam spill window duration. The two sub-event event is likely to come from the decay of a stopping muon. The range shown is a subset of the $19.2 \mu \mathrm{s}$ DAQ window.

Subevents are defined as groups of at least ten hits separated by less than $10 \mathrm{~ns}$ from one another. A typical $\nu_{\mu}$ CCQE interaction produces a pattern of time activity with two subevents, one due to the muon created in the interaction, which suffers ionization 
energy loss and stops, and a second subevent from its decay Michel electron. An example is shown in Figure 2.11.

The activity in the tank during the DAQ window is substantially increased when the proton beam is on. This is shown in Figure 2.12 where the $1.6 \mu$ s beam spill from the Booster beam is clearly seen even without any selection cuts.

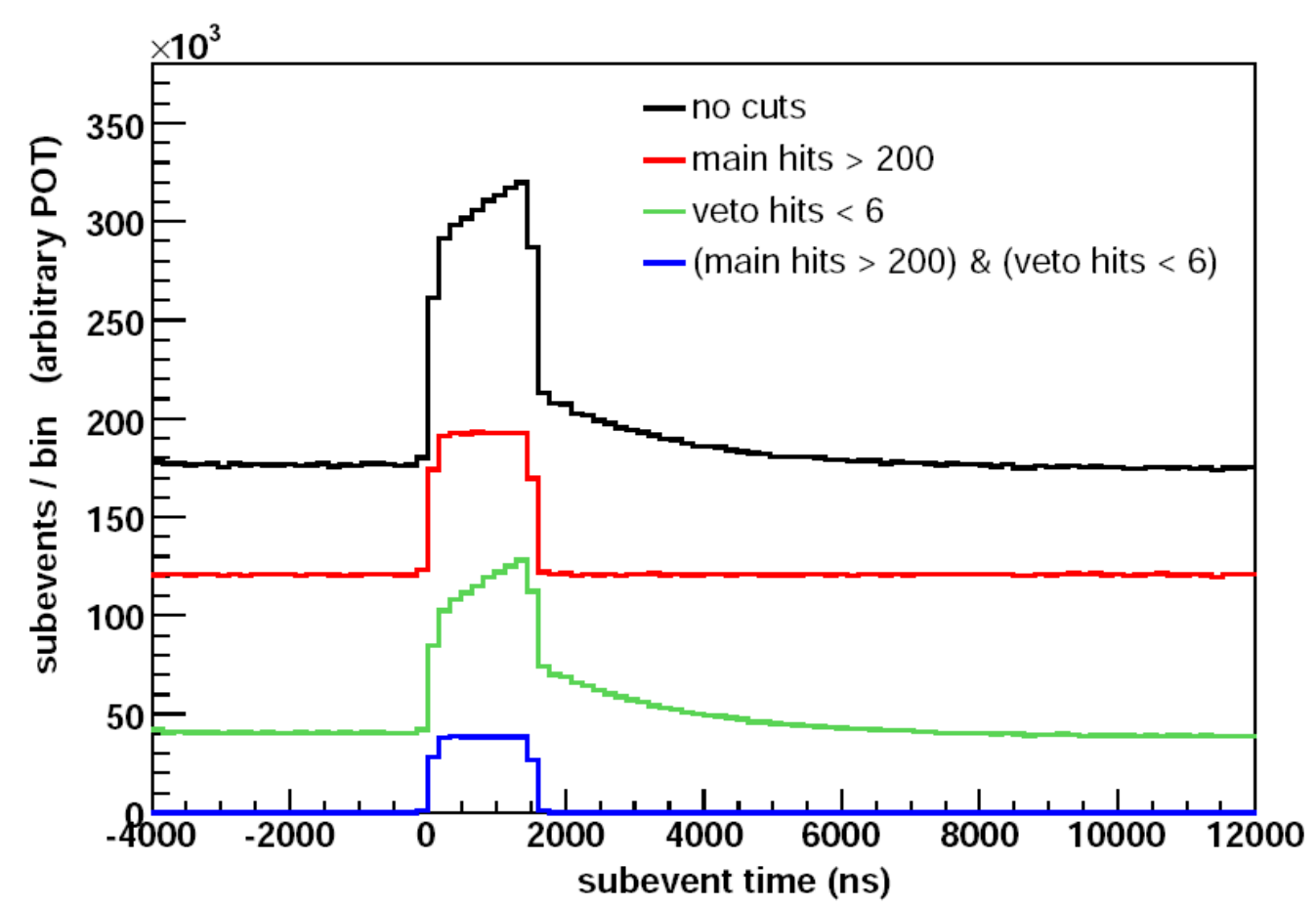

Figure 2.12: PMT hit multiplicity cuts applied to select neutrino interactions and reject cosmogenic backgrounds in MiniBooNE. Background rejection is 99.9\%. Taken from [180].

A clean sample of neutrino interactions is selected by requiring low PMT multiplicity in the veto region and a minimum PMT multiplicity in the main tank. Cosmic ray muons that enter the tank during the $19.2 \mu$ s DAQ window produce a significant amount of light in the veto and can be eliminated efficiently with a low veto activity requirement $\left(N_{\text {veto }}<6\right)$. Residual cosmogenic activity is produced when a cosmic muon penetrates the tank when the DAQ window is not open, but decays after the 
window has opened. Michel electrons, having energies below $52.8 \mathrm{MeV}$, have a similarly compact MT hit distribution range extending up to 200 PMT hits in the main tank.

In Figure 2.12 the distribution of times of all subevents relative to the start of the beam spill is shown. Without any cuts (topmost black histogram) the excess due to neutrino interactions is evident. The width of the excess correseponds with the duration of the beam spill $(1.6 \mu \mathrm{s})$. Beam-induced Michel electrons accumulate early in the spill and cause the raising slope and subsequent exponential decay. Events with energies above the Michel endpoint populate the red histogram (second from top), as well as cosmic ray backgrounds contributing to the flat component. The green histogram (third from top) shows the effect of a low veto hit multiplicity requirement. The residual exponential decay corresponds to the Michels whose parent muon penetrates the tank while the DAQ window is closed. Finally, the blue histogram (fourth from top) shows how the combination of the multiplicity cuts in the main tank and the veto isolate a pure sample of neutrino interactions within the beam window. 


\section{Chapter 3}

\section{Experiment Simulation and Reconstruction algorithms}

The simulation of the experiment can be broken down into three main components: I) Beam Simulation, II) Neutrino interactions and final state generation III) Detector simulation (light propagation and electronics response). Details on the beam simulation can be found in [182]. Only a cursory description is given here.

\subsection{Beam simulation}

A GEANT4 $[123,124]$ based program is first used to simulate the production of secondary particles in a proton-beryllium interaction at incident proton beam momentum of $8.9 \mathrm{GeV} / c$. The four most relevant types of secondaries for neutrino flux calculations are $\pi^{+}, \pi^{-}, K^{+}, K_{L}^{0}$, followed by $p$ and $n$, all of which are produced in inelastic interactions of the primary proton beam with the Be target. $K^{-}$are not simulated since their contribution to the neutrino flux is at least two orders of magnitude smaller than that of $K^{+}$. The production simulation of the first four types and the 
estimation of the corresponding uncertainties are based on parameterizations of the meson production non-invariant double-differential cross section $\frac{d^{2} \sigma}{d p d \Omega}$ extracted from existing measurements of either the non-invariant cross section itself or the invariant cross section $E \frac{d^{3} \sigma}{d p^{3}}$ (related by $\frac{d^{2} \sigma}{d p d \Omega}=\frac{p^{2}}{E} E \frac{d^{3} \sigma}{d p^{3}}$ ). The simulation of $p$ and $n$ production and is based on production cross-sections obtained from the MARS15 Monte Carlo $[125,126]$. The neutrino flux is then simulated with a FORTRAN based tool that generates kinematic distributions for neutrinos from the decays of the simulated mesons and muons, extrapolating it to the detector location.

\subsubsection{Particle production parameterizations}

Data from experiments measuring particle production by protons of various energies on many different nuclear targets data have been historically used to study the phenomenology of particle production and have led to several scaling laws and quark counting rules. Table 3.1 shows the production processes of various mesons in $p+p$ interactions and their threshold production energies.

Table 3.1: Threshold production channels for $p+p$ production of various mesons. $M_{F S}$ is the minimum mass of the final state in the second column. The table shows the threshold energy in the CM frame $\sqrt{s_{\mathrm{th}}}$ and in the lab frame $E_{\mathrm{th}}^{\text {Beam }}$

\begin{tabular}{clllccc}
\hline $\begin{array}{c}\text { Produced } \\
\text { Hadron }\end{array}$ & $\begin{array}{l}\text { Final } \\
\text { State }\end{array}$ & $\begin{array}{l}\text { Production } \\
\text { Process }\end{array}$ & $\begin{array}{l}\text { Quark } \\
\text { Process }\end{array}$ & $\begin{array}{c}M_{F S} \\
\left(\mathrm{GeV} / c^{2}\right)\end{array}$ & $\begin{array}{c}\sqrt{s_{\mathrm{th}}} \\
(\mathrm{GeV})\end{array}$ & $\begin{array}{c}E_{\mathrm{th}}^{\text {Beam }} \\
\mathrm{GeV}\end{array}$ \\
\hline$\pi^{+}$ & $p n \pi^{+}$ & $p \rightarrow n \pi^{+}$ & $u u d \rightarrow u \bar{d}$ & 1.878 & 2.018 & 1.233 \\
$\pi^{-}$ & $p p \pi^{+} \pi^{-}$ & $p \rightarrow p \pi^{+} \pi^{-}$ & $u u d \rightarrow d \bar{u}$ & 2.016 & 2.156 & 1.54 \\
$K^{+}$ & $\Lambda^{0} p K^{+}$ & $p \rightarrow \Lambda^{0} K^{+}$ & $u u d \rightarrow u \bar{s}$ & 2.053 & 2.547 & 2.52 \\
$K^{-}$ & $p p K^{+} K^{-}$ & $p \rightarrow p K^{+} K^{-}$ & $u u d \rightarrow s \bar{u}$ & 2.37 & 2.864 & 3.434 \\
$K^{0}$ & $p \Sigma^{+} K^{0}$ & $p \rightarrow p \Sigma^{+} K^{0}$ & $u u d \rightarrow d \bar{s}$ & 2.13 & 2.628 & 2.743 \\
\hline
\end{tabular}


Sanford and Wang (SW) [121] proposed the following parameterization of the double-differential cross section of particle production:

$$
{\frac{d^{2} \sigma}{d \Omega d p}}^{\mathrm{SW}}=c_{1} p^{c_{2}}\left(1-\frac{p}{p_{B}-c_{9}}\right) \exp \left(-c_{3} \frac{p^{c_{4}}}{p_{B}^{c_{5}}}-c_{6}\left(p-c_{7} p_{B} \cos ^{c_{8}} \theta\right) \theta\right)
$$

where $p_{B}$ is the incident proton beam momentum, $\theta$ and $p$ are the scattering angle and momentum of the produced meson, and $c_{1}, \ldots, c_{9}$ are parameters to be determined from experiments. Wang [122] found coefficients satisfying $c_{2} \simeq 1 / 2, c_{4} \simeq c_{5} \simeq 5 / 3$, and $c_{7} \simeq 0$, from beam momenta between $10 \mathrm{GeV} / c$ and $70 \mathrm{GeV} / c$ on Be. With these substitutions, defining $X \equiv p / p_{B}$, and approximating $p_{t} \simeq p \theta$, the SW formula Eq.(3.1) becomes

$$
{\frac{d^{2} \sigma}{d p d \Omega}}^{\mathrm{SW}}=c_{1} p_{B}^{1 / 2} F(X) \exp \left(-c_{6} p_{t}\right), \text { with } \quad F(X)=X^{1 / 2}(1-X) \exp \left(-c_{3} X^{5 / 3}\right),
$$

from where we see that the non-invariant double-differential cross section and angle of mesons produced at two different momenta $p_{B}$ and $p_{B}^{\prime}$ satisfy the scaling laws:

$$
\frac{d^{2} \sigma\left(p_{B}^{\prime}\right)}{d p d \Omega}=\left(\frac{p_{B}^{\prime}}{p_{B}}\right)^{1 / 2} \times \frac{d^{2} \sigma\left(p_{B}^{\prime}\right)}{d p d \Omega}, \text { and } \quad \theta^{\prime}=\frac{p_{B}}{p_{B}^{\prime}} \times \theta .
$$

The more complex functional form in Eq.(3.1) suits better the production of pions at the MiniBooNE beam momentum of $8.9 \mathrm{GeV} / c$. Parameters $c_{1}, c_{3}, c_{5}$ and $c_{9}$ determine the change in cross section with incident beam momentum, while $c_{2}$ and $c_{4}$ relate to the variation with the outgoing pion momentum, and parameters $c_{6}, c_{7}$, and $c_{8}$ relate to the variation with the pion production angle.

Other parameterizations are possible as well. For example, Feynman [127] proposed a theoretical model where the invariant cross section is only a function of the transverse momentum $p_{t}$ and the variable $x_{F}$ :

$$
E \frac{d^{3} \sigma}{d p^{3}}=A F\left(x_{F}\right) G\left(p_{t}\right) \Rightarrow \frac{d^{2} \sigma}{d p d \Omega}=\frac{p^{2}}{E} E \frac{d^{3} \sigma}{d p^{3}}=\frac{p^{2}}{E} A F\left(x_{F}\right) G\left(p_{t}\right)
$$

where $A$ is a normalization constant, $x_{F} \equiv \frac{p_{z}^{c m}}{p_{\|}^{c m} \text { max }}$, and $p_{\|}^{c m m a x} \approx \sqrt{s} / 2$, with $\sqrt{s}$, the center of mass energy in the collision. This scaling has been previously tested for 
large incident beam momenta $P_{B}>50 \mathrm{GeV}$, but it gives a good description of $K^{+}$ production data at lower momenta relevant for MiniBooNE, as has been shown by Shaevitz [132].

\section{Simulation of $\pi^{+}$production}

The $\pi^{+}$production cross section is described with a Sanford-Wang (SW) parameterization like that in Eq.(3.1). The HARP experiment [129] at CERN measured the $\pi^{+}$production double-differential cross section in $p$-Be interactions with a replica of the MiniBooNE target at the incident beam momentum of $8.9 \mathrm{GeV} / c$. To obtain a better measure of the uncertainty on the SW fit parameters it was decided to use data from the E910 [128] experiment at two incident beam momenta, one above and one below that of MiniBooNE's beam. E910 data also has the favorable feature of having a broader coverage on the production angle $\theta$.

Data from HARP [130] at $p_{B}=8.9 \mathrm{GeV} / c$, and E910 at $p_{B}=6.4 \mathrm{GeV} / c$ and $p_{B}=12.3 \mathrm{GeV} / c$ are used in a fit to determine the coefficients $c_{1}$ through $c_{8}\left(c_{9}\right.$ is kept fixed at 1.0 for $\pi^{+}$production fits) via a $\chi^{2}$ minimization fit that takes into account the bin-to bin correlations of the measurements. Details on the fit performed to extract the parameter values can be found in [115]. In this procedure it was found that the parameters $c_{3}$ and $c_{5}$ are completely correlated, therefore parameter $c_{3}$ was fixed at 1.0 also. The result is expressed as a central value for the parameters $c_{1}, \ldots, c_{8}$, and a covariance matrix $\mathcal{M}^{\pi^{+}}$for them, shown in table 3.2.

\section{Simulation of $\pi^{-}$production}

The production of $\pi^{-}$is also described with a SW parameterization like that of Eq.(3.1). In this case parameters $c_{1}$ through $c_{8}$ are allowed to float while keeping $c_{9}$ 


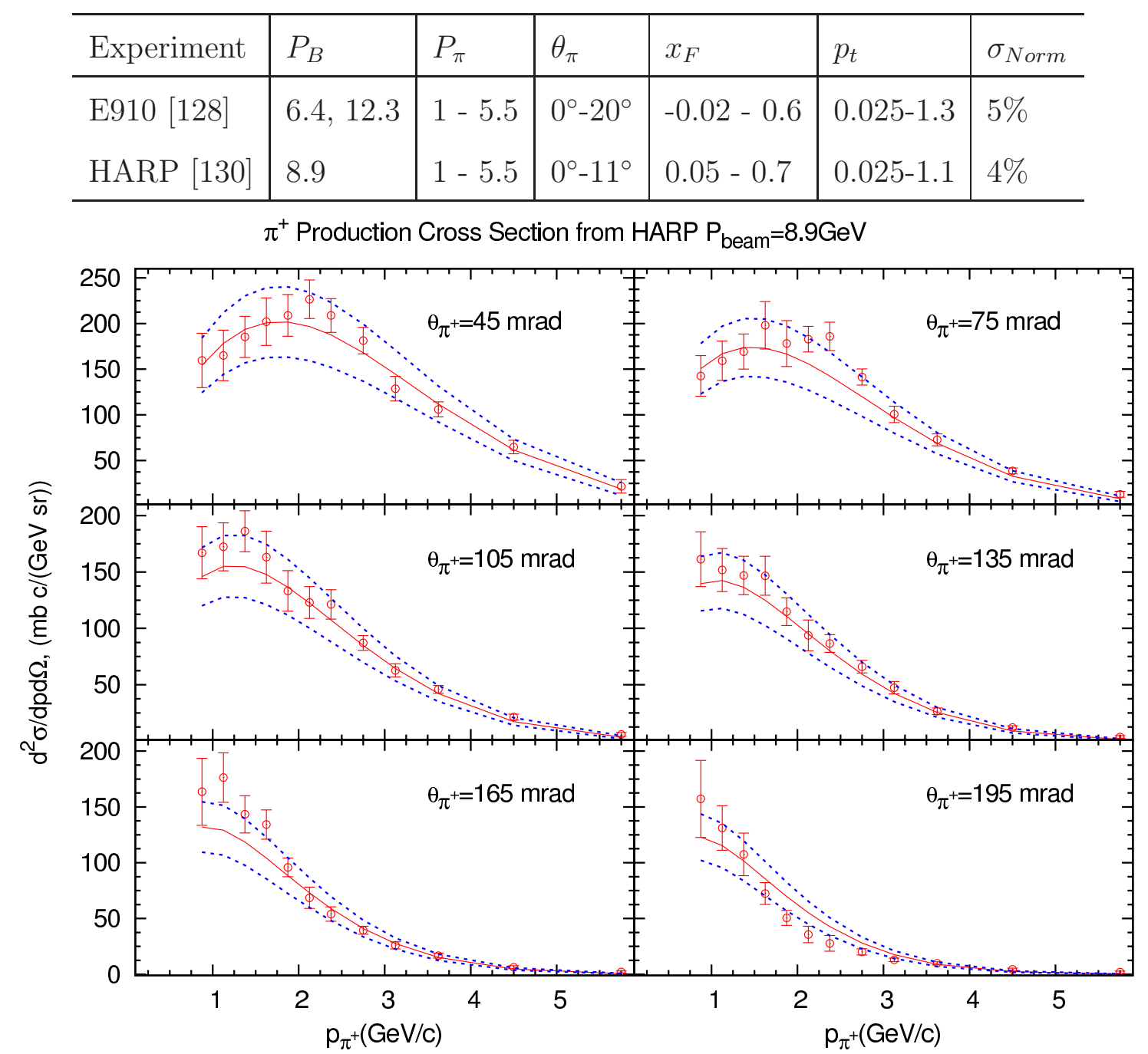

Figure 3.1: Top: Summary of $p B e \rightarrow \pi^{+} X$ experiments used in the determination of the parameterization in Eq.(3.1). $P_{B}, P_{K}$ and $p_{t}$ are given in GeV/c. Bottom: SanfordWang function (red solid curve) and HARP data (red points). The blue dotted curves were obtained from 1000 draws from the multivariate Gaussian implied by the $7 \times 7$ covariance matrix in Table 3.2. The Bands indicate the RMS in the $\pi^{+}$production cross section for each $\left(p_{\pi}, \theta\right)$. Taken from [115]. 
Table 3.2: The central values and covariance matrix of SW parameters describing $\pi^{+}$ production in MiniBooNE. Extracted from fits to HARP [129] and E910 [128] $\pi^{+}$production data. Parameters $c_{3}$ and $c_{9}$ have been fixed at 1.0.

\begin{tabular}{c|ccccccc}
$\mathrm{CV}$ & 220.7 & 1.080 & 1.978 & 1.320 & 5.572 & 0.08678 & 9.686 \\
\hline \hline $\mathcal{M}_{i j}^{\pi^{+}}$ & $c_{1}$ & $c_{2}$ & $c_{4}$ & $c_{5}$ & $c_{6}$ & $c_{7}$ & $c_{8}$ \\
\hline$c_{1}$ & 1707.22 & 1.14601 & -17.6455 & -15.9683 & -8.80997 & -0.73472 & -60.8160 \\
$c_{2}$ & 1.14601 & 0.03963 & -0.10719 & -0.09928 & 0.03249 & 0.00069 & -0.07772 \\
$c_{4}$ & -17.6455 & -0.10719 & 0.59447 & 0.50491 & 0.06546 & 0.00251 & 0.19795 \\
$c_{5}$ & -15.9683 & -0.09928 & 0.50491 & 0.44109 & 0.05684 & 0.00250 & 0.22709 \\
$c_{6}$ & -8.80997 & 0.03249 & 0.06546 & 0.05684 & 0.20664 & 0.00466 & 0.10310 \\
$c_{7}$ & -0.73472 & 0.00069 & 0.00251 & 0.00250 & 0.00466 & 0.00049 & 0.06405 \\
$c_{8}$ & -60.8160 & -0.07772 & 0.19795 & 0.22709 & 0.10310 & 0.06405 & 16.01887 \\
\hline
\end{tabular}

fixed at 1.0 in a $\chi^{2}$ minimization fit (technique described in [181]). The results used in this thesis are shown in table 3.3 .

As we will see the contribution from this source to the final uncertainty on the oscillations analysis presented in this thesis is negligible small, since only less than $1 \%$ of the neutrino flux originates from $\pi^{-}$decays when the experiment is running in neutrino mode (normal horn polarity). This will not be the case in an oscillations search in the anti-neutrino mode (reversed horn polarity), where the dominant contribution of the events in the tank will originate from the decays of $\pi^{-}$.

\section{Simulation of $K^{+}$production}

A parameterization based on Feynman scaling [127] was developed by M. H. Shaevitz [131] to fit various external data sets from experiments on $K^{+}$production. The 
Table 3.3: The central values and covariance matrix of SW parameters describing $\pi^{-}$ production in MiniBooNE. Extracted from $\pi^{-}$production HARP [129] and E910 [128] data. Parameter $c_{9}$ has been fixed at 1.0.

\begin{tabular}{c|cccccccc}
\hline $\mathrm{CV}$ & 237.2 & 0.8986 & 4.521 & 1.154 & 1.105 & 4.224 & 0.06613 & 9.961 \\
\hline \hline $\mathcal{M}_{i j}^{\pi^{-}}$ & $c_{1}$ & $c_{2}$ & $c_{3}$ & $c_{4}$ & $c_{5}$ & $c_{6}$ & $c_{7}$ & $c_{8}$ \\
\hline$c_{1}$ & 2309.02 & 3.0489 & 7.0802 & -5.5568 & -5.9875 & -5.7957 & -0.7692 & -63.227 \\
$c_{2}$ & 3.0489 & 0.0258 & -0.0168 & -0.0095 & -0.0179 & 0.0229 & $-4.43 \mathrm{E}-05$ & -0.1989 \\
$c_{3}$ & 7.0802 & -0.0168 & 1.0963 & -0.0240 & 0.0922 & -0.0272 & -0.0042 & -0.2816 \\
$c_{4}$ & -5.5568 & -0.0095 & -0.0240 & 0.0272 & 0.0227 & 0.0096 & 0.0008 & 0.0308 \\
$c_{5}$ & -5.9875 & -0.0179 & 0.0922 & 0.0227 & 0.0356 & 0.0110 & 0.0007 & 0.0340 \\
$c_{6}$ & -5.7957 & 0.0229 & -0.0272 & 0.0096 & 0.0110 & 0.1496 & 0.0031 & -0.4283 \\
$c_{7}$ & -0.7692 & $-4.43 \mathrm{E}-05$ & -0.0042 & 0.0008 & 0.0007 & 0.0031 & 0.0005 & 0.0741 \\
$c_{8}$ & -63.227 & -0.1989 & -0.2816 & 0.0308 & 0.0340 & -0.4283 & 0.0741 & 27.513 \\
\hline
\end{tabular}

parameterization used in this case is:

$$
\frac{d^{2} \sigma}{d p d \Omega}=\left(\frac{p_{K}^{2}}{E_{K}}\right) c_{1} \exp \left[-c_{3}\left|x_{F}\right|^{c_{4}}-c_{7}\left|p_{t} \times x_{F}\right|^{c_{6}}-c_{2} p_{t}-c_{5} p_{t}^{2}\right]
$$

where $p_{K}$ and $E_{K}$ are the momentum and energy of the outgoing $K^{+}$respectively, and the parameters $c_{1}, \ldots, c_{7}$ are determined from fits to external data. This parameterization proved to be superior to the SW parameterization in describing the available $K^{+}$production data listed in Figure 3.2, The data sets in Figure 3.2 where scaled to a common beam momentum of $8.9 \mathrm{GeV} / c$ and seen to respect the scaling hypothesis of Eq.(3.2).

\section{Simulation of $K^{0}$ production}

Data on $p B e \rightarrow K^{0} X$ from the E910 experiment [142] at incident beam momenta of 12.3 $\mathrm{GeV} / c$ and $17.6 \mathrm{GeV} / c$, and Abe [143] at beam momentum of $12.0 \mathrm{GeV} / c$ were 


\begin{tabular}{l|l|l|l|l|l|l}
\hline Experiment & $P_{B}$ & $P_{K}$ & $\theta_{K}$ & $x_{F}$ & $p_{t}$ & $\sigma_{\text {Norm }}$ \\
\hline Abbott [133] & 14.6 & $2-8$ & $20^{\circ}-30^{\circ}$ & $-0.12-0.07$ & $0.2-0.7$ & $10 \%$ \\
Aleshin [140] & 9.5 & $3-6.5$ & $3.5^{\circ}$ & $0.3-0.8$ & $0.2-0.4$ & $10 \%$ \\
Allaby [134] & 19.2 & $3-16$ & $0^{\circ}-7^{\circ}$ & $0.3-0.9$ & $0.1-1.0$ & $15 \%$ \\
Dekkers [135] & $18.8,23.1$ & $4-12$ & $0^{\circ}, 5^{\circ}$ & $0.1-0.5$ & $0.0-1.2$ & $20 \%$ \\
Eichten [139] & 24.0 & $4-18$ & $0^{\circ}-6^{\circ}$ & $0.1-0.8$ & $0.1-1.2$ & $20 \%$ \\
Vorontsov [141] & 10.1 & $1-4.5$ & $3.5^{\circ}$ & $0.03-0.5$ & $-0.1-0.25$ & $25 \%$ \\
\hline
\end{tabular}

$\mathrm{K}^{+}$Production Data and Fit (Scaled to $\mathrm{P}_{\text {beam }}=8.89 \mathrm{GeV}$ )
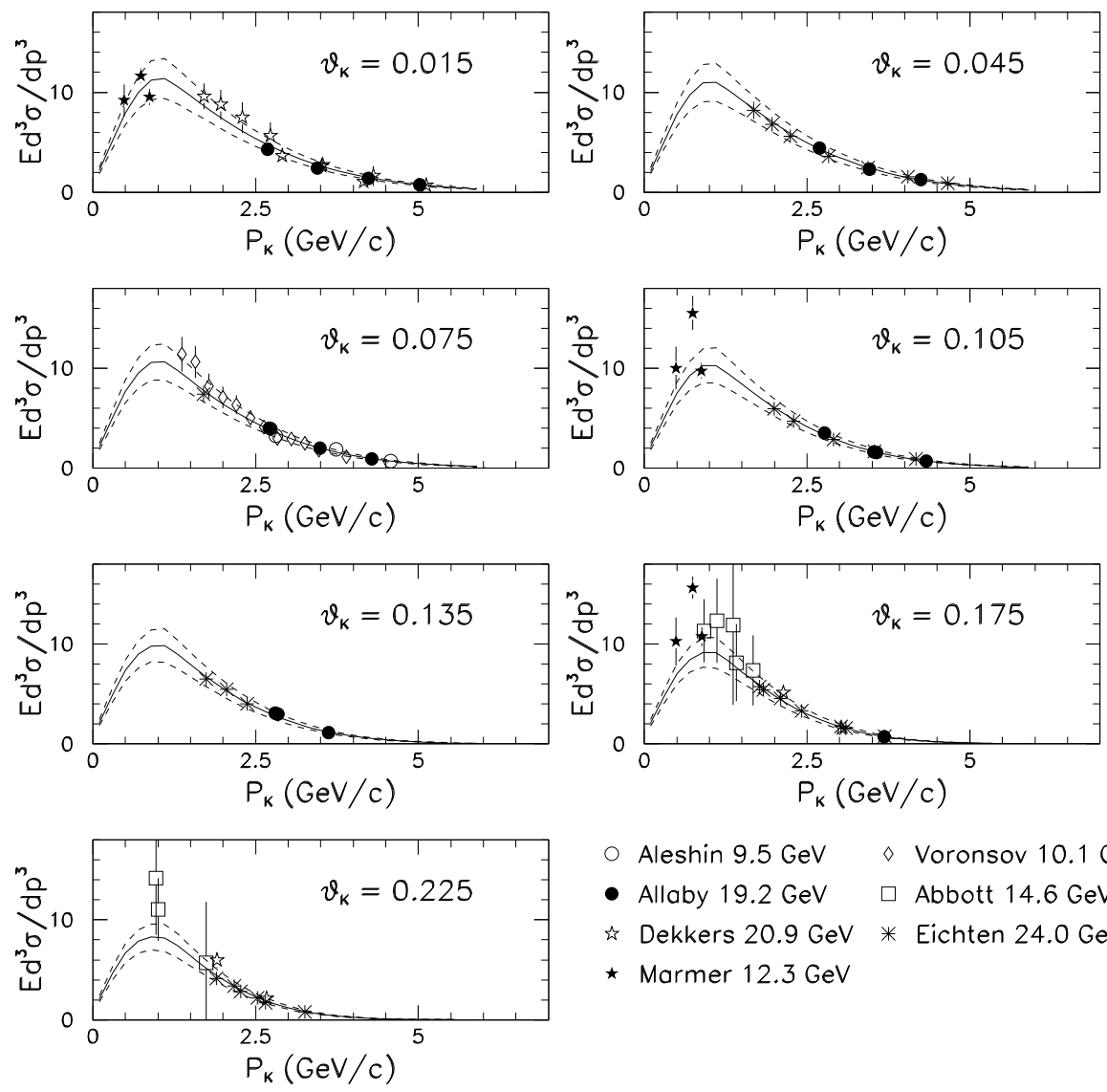

Aleshin $9.5 \mathrm{GeV} \diamond$ Voronsov $10.1 \mathrm{GeV}$

- Allaby $19.2 \mathrm{GeV} \square$ Abbott $14.6 \mathrm{GeV}$

¿ Dekkers $20.9 \mathrm{GeV}$ * Eichten $24.0 \mathrm{GeV}$

$\star$ Marmer $12.3 \mathrm{GeV}$

Figure 3.2: Top: Summary of recent $p B e \rightarrow K^{+} X$ experiments used in the determination of the parameterization in Eq.(3.3). $P_{B}, P_{K}$ and $p_{t}$ are given in $\mathrm{GeV} / c$. Bottom: Comparison of various data sets (after Feynman scaling) with the Feynman Scaling parameterization (solid curve). The error bands (dashed curves) correspond to the uncertainties in Table 3.4. 
Table 3.4: The central values (CV) and covariance matrix for the parameters of the Feynman Scaling parameterization describing $K^{+}$(Eq.3.3) production in MiniBooNE as calculated by Shaevitz [131].

\begin{tabular}{c|ccccccc}
\hline $\mathrm{CV}$ & 11.70 & 0.88 & 4.77 & 1.51 & 2.21 & 2.17 & 1.51 \\
\hline \hline $\mathcal{M}_{i j}^{K^{+}}$ & $c_{1}$ & $c_{2}$ & $c_{3}$ & $c_{4}$ & $c_{5}$ & $c_{6}$ & $c_{7}$ \\
\hline$c_{1}$ & 1.094046 & 0.05017 & $2.99 \mathrm{E}-03$ & -0.03316 & -0.03745 & 0.125194 & 0.074319 \\
$c_{2}$ & 0.05017 & 0.016104 & $1.39 \mathrm{E}-03$ & $-1.44 \mathrm{E}-03$ & -0.01264 & 0.032194 & 0.021996 \\
$c_{3}$ & $2.99 \mathrm{E}-03$ & $1.39 \mathrm{E}-03$ & $7.47 \mathrm{E}-03$ & $2.06 \mathrm{E}-03$ & $1.93 \mathrm{E}-03$ & 0.013534 & $-3.34 \mathrm{E}-03$ \\
$c_{4}$ & -0.03316 & $-1.44 \mathrm{E}-03$ & $2.06 \mathrm{E}-03$ & $3.46 \mathrm{E}-03$ & $2.03 \mathrm{E}-03$ & $-4.11 \mathrm{E}-03$ & $-6.28 \mathrm{E}-03$ \\
$c_{5}$ & -0.03745 & -0.01264 & $1.93 \mathrm{E}-03$ & $2.03 \mathrm{E}-03$ & 0.014637 & -0.01544 & -0.02444 \\
$c_{6}$ & 0.125194 & 0.032194 & 0.013534 & $-4.11 \mathrm{E}-03$ & -0.01544 & 0.181522 & 0.126181 \\
$c_{7}$ & 0.074319 & 0.021996 & $-3.34 \mathrm{E}-03$ & $-6.28 \mathrm{E}-03$ & -0.02444 & 0.126181 & 0.159265 \\
\hline
\end{tabular}

used in a fit by Shaevitz [132] to determine the Sanford-Wang parameters in Eq.(3.1) that best describe the data. The characteristics of the data sets are illustrated in Figure 3.3.

\subsubsection{Neutrino flux simulation}

Following the simulation of particle production, individual secondaries are tracked as they interact with the materials of the various elements in the beamline geometry (described in detail in [182]), some producing other secondaries. As they exit the target and enter the magnetic field region of the horn, charged secondaries modify their trajectories according to a static and azimuthal $\sim 1 / r$ magnetic field. Particles are tracked across the materials of the target, horn and the decay pipe simulating electromagnetic interactions, ionization energy loss, multiple Coulomb scattering and ultimately meson decay. 


\begin{tabular}{l|l|l|l|l|l|l}
\hline Experiment & $P_{B}$ & $P_{K}$ & $\theta_{K}$ & $x_{F}$ & $p_{t}$ & $\sigma_{\text {Norm }}$ \\
\hline E910 [142] & $12.3,17.6$ & $0.5-2.5$ & $4^{\circ}-32^{\circ}$ & $-0.35-0.45$ & $0.25-1.0$ & $5 \%$ \\
Abe [143] & 12.0 & $5.52-9.7$ & $3.5^{\circ}, 5^{\circ}$ & $0.5-0.82$ & $0.35-0.85$ & $20 \%$ \\
\hline
\end{tabular}

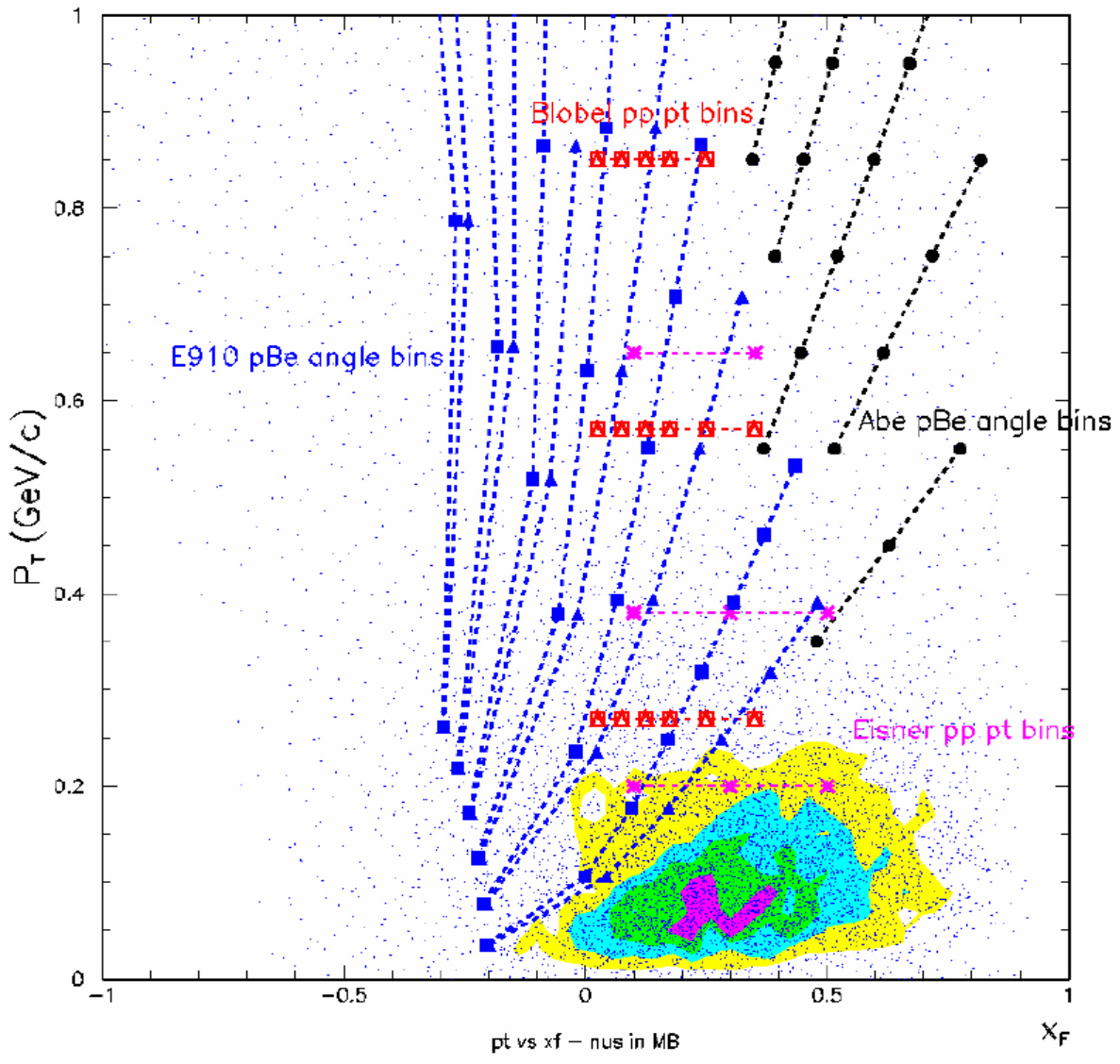

Figure 3.3: Top: Summary of $p B e \rightarrow K^{0} X$ experiments used in the determination of the parameterization in Eq.(3.1). $P_{B}, P_{K}$ and $p_{t}$ are given in $\mathrm{GeV} / c$. Bottom: Plot of $p_{t} v s . x_{F}$ from various experiments (squares with dashed lines) compared to the MiniBooNE Beam Monte Carlo prediction for $K^{0}$ with $\nu_{e}$ entering the MiniBooNE detector (scattered points and contours). Taken from [132]. 
Table 3.5: The central values (CV) and covariance matrix for the parameters of the SanfordWang parameterization describing $K^{0}$ (Eq.3.1) production in MiniBooNE as calculated by Shaevitz [132].

\begin{tabular}{c|ccccccccc}
\hline $\mathrm{CV}$ & 15.13 & 1.975 & 4.084 & 0.9277 & 0.7306 & 4.362 & $4.79 \mathrm{E}-02$ & 13.3 & 1.278 \\
\hline \hline $\mathcal{M}_{i j}^{K^{0}}$ & $c_{1}$ & $c_{2}$ & $c_{3}$ & $c_{4}$ & $c_{5}$ & $c_{6}$ & $c_{7}$ & $c_{8}$ & $c_{9}$ \\
\hline$c_{1}$ & 32.302 & -0.0969 & 0.8215 & -0.1018 & -0.2124 & -0.8902 & -0.1333 & 16.552 & -1.7893 \\
$c_{2}$ & -0.0969 & 0.0957 & 0.0325 & 0.0013 & -0.0130 & 0.0884 & -0.0003 & -1.5364 & -0.2156 \\
$c_{3}$ & 0.8215 & 0.0325 & 0.5283 & -0.0192 & 0.0227 & -0.0033 & -0.0024 & 0.0391 & -0.0802 \\
$c_{4}$ & -0.1018 & 0.0013 & -0.0192 & 0.0084 & 0.0040 & 0.0007 & -0.0004 & -0.0144 & -0.0730 \\
$c_{5}$ & -0.2124 & -0.0130 & 0.0227 & 0.0040 & 0.0098 & 0.0029 & 0.0003 & -0.0578 & 0.0297 \\
$c_{6}$ & -0.8902 & 0.0884 & -0.0033 & 0.0007 & 0.0029 & 0.3599 & 0.0038 & -4.7514 & -0.1577 \\
$c_{7}$ & -0.1333 & -0.0003 & -0.0024 & -0.0004 & 0.0003 & 0.0038 & 0.0010 & 0.0581 & 0.0069 \\
$c_{8}$ & 16.552 & -1.5364 & 0.0391 & -0.0144 & -0.0578 & -4.7514 & 0.0581 & 130.201 & 1.2222 \\
$c_{9}$ & -1.7893 & -0.2156 & -0.0802 & -0.0730 & 0.0297 & -0.1577 & 0.0069 & 1.2222 & 2.9480 \\
\hline
\end{tabular}

The flux of each neutrino type per proton delivered to the target is shown in Figure 3.4. Neutrinos are produced by the decay of mesons that are produced somewhere along the chain that starts with the production of a secondary particle in the target. The plots in the figure show the contributions from the chains starting with the production of $\pi^{ \pm}, K^{+}, K_{L}^{0}$, or nucleons, producing in the end a neutrino of a given flavor.

\section{Hadronic interactions}

The hadronic interactions of primary protons as well as secondary $p, n, \pi^{+}$, and $\pi^{-}$within the target and the horn material are handled using parameterizations of the momentum dependence of the $(p / n)$-Nucleus and $\pi^{ \pm}$-Nucleus total, inelastic and quasi-elastic cross-sections $\left(\sigma_{\mathrm{TOT}}, \sigma_{\mathrm{INE}}, \sigma_{\mathrm{QE}}\right.$ respectively) [144], related by

$$
\sigma_{\mathrm{TOTAL}}=\sigma_{\mathrm{INE}}+\sigma_{\mathrm{ELA}} \quad \text { and } \quad \sigma_{\mathrm{INE}}=\sigma_{\mathrm{PROD}}+\sigma_{\mathrm{QE}}
$$




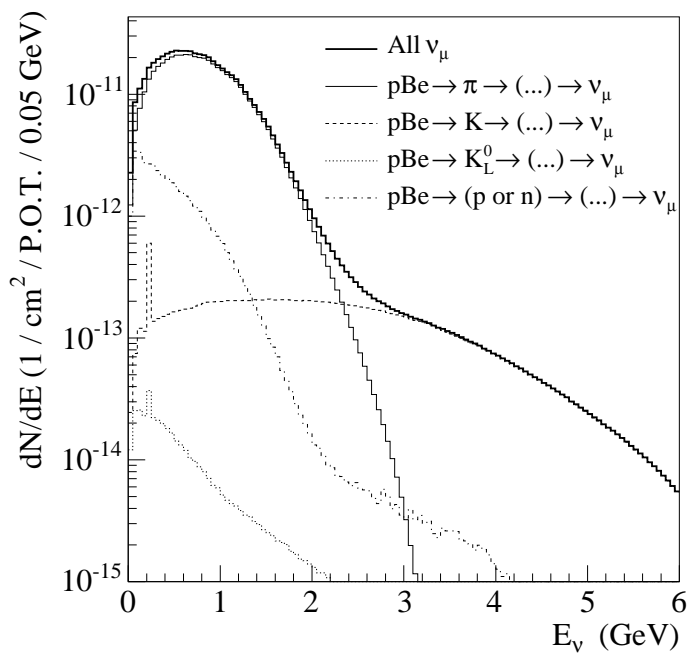

(a) $\nu_{\mu}$ flux.

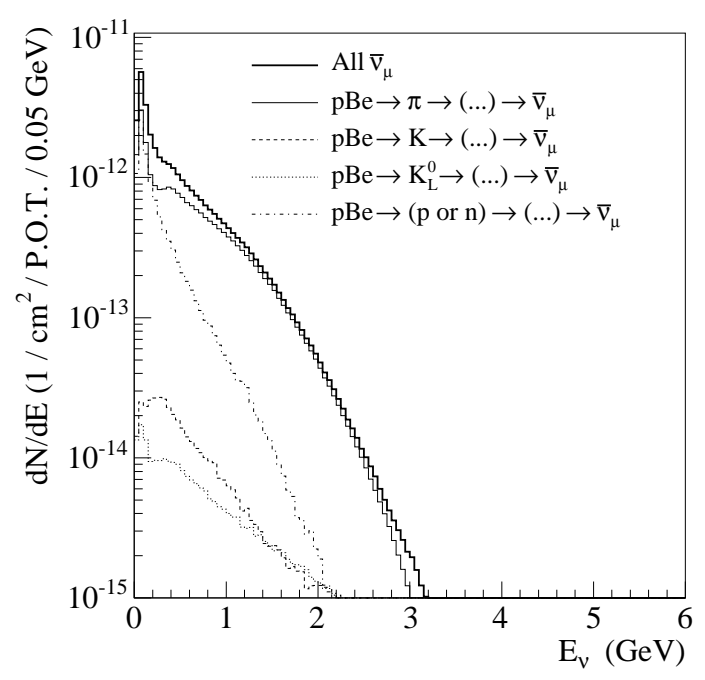

(c) $\bar{\nu}_{\mu}$ flux.

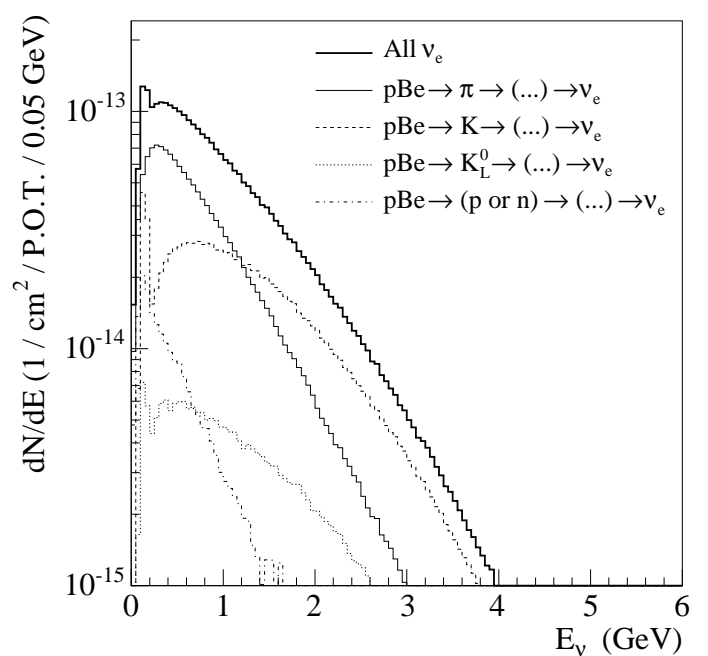

(b) $\nu_{e}$ flux.

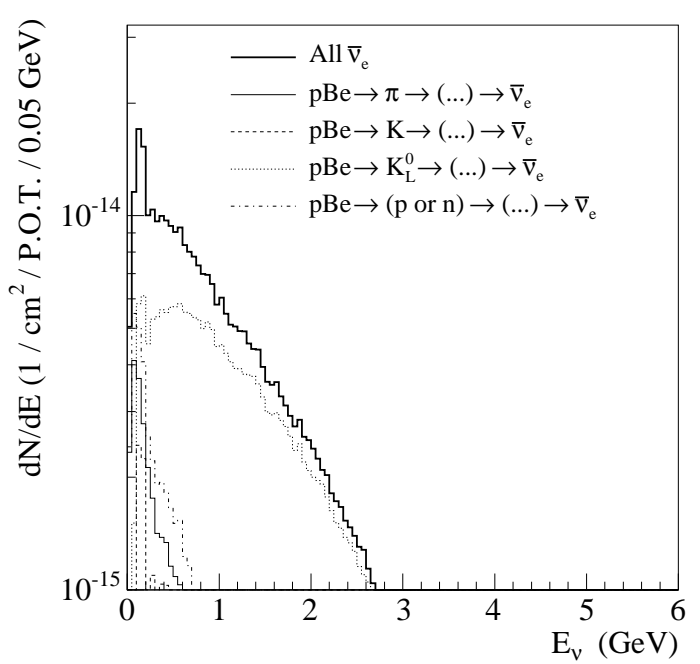

(d) $\bar{\nu}_{e}$ flux.

Figure 3.4: Predicted flux in the MiniBooNE detector as a function of neutrino energy for each neutrino type. The components from different secondary particles produced at the target are shown. The percent contribution of these various sources is shown in table 3.6. 
Table 3.6: Predicted contributions to the neutrino fluxes in Figure 3.4 per secondary particle produced at the target. Each neutrino type comes from a chain initiated by the indicated process.

\begin{tabular}{|c|c|c|}
\hline Neutrino flavor & Process & Contribution \\
\hline \multirow{4}{*}{$\nu_{\mu}(93.5 \%)$} & $\mathrm{pBe} \rightarrow \pi^{ \pm} \rightarrow(\ldots) \rightarrow \nu_{\mu}$ & $89.5 \%$ \\
\hline & $\mathrm{pBe} \rightarrow K^{ \pm} \rightarrow(\ldots) \rightarrow \nu_{\mu}$ & $2.8 \%$ \\
\hline & $\mathrm{pBe} \rightarrow K_{L}^{0} \rightarrow(\ldots) \rightarrow \nu_{\mu}$ & $<0.1 \%$ \\
\hline & $\mathrm{pBe} \rightarrow \mathrm{p}$ or $\mathrm{n} \rightarrow(\ldots) \rightarrow \nu_{\mu}$ & $7.7 \%$ \\
\hline \multirow{4}{*}{$\nu_{e}(0.5 \%)$} & $\mathrm{pBe} \rightarrow \pi^{ \pm} \rightarrow(\ldots) \rightarrow \nu_{e}$ & $48.8 \%$ \\
\hline & $\mathrm{pBe} \rightarrow K^{ \pm} \rightarrow(\ldots) \rightarrow \nu_{e}$ & $36.8 \%$ \\
\hline & $\mathrm{pBe} \rightarrow K_{L}^{0} \rightarrow(\ldots) \rightarrow \nu_{e}$ & $7.0 \%$ \\
\hline & $\mathrm{pBe} \rightarrow \mathrm{p}$ or $\mathrm{n} \rightarrow(\ldots) \rightarrow \nu_{e}$ & $7.4 \%$ \\
\hline \multirow{5}{*}{$\bar{\nu}_{\mu}(5.9 \%)$} & $\mathrm{pBe} \rightarrow \pi^{ \pm} \rightarrow(\ldots) \rightarrow \bar{\nu}_{\mu}$ & $66.5 \%$ \\
\hline & $\mathrm{pBe} \rightarrow K^{ \pm} \rightarrow(\ldots) \rightarrow \bar{\nu}_{\mu}$ & $1.3 \%$ \\
\hline & $\mathrm{pBe} \rightarrow K_{L}^{0} \rightarrow(\ldots) \rightarrow \bar{\nu}_{\mu}$ & $0.7 \%$ \\
\hline & $\mathrm{pBe} \rightarrow \mathrm{p}$ or $\mathrm{n} \rightarrow(\ldots) \rightarrow \bar{\nu}_{\mu}$ & $31.0 \%$ \\
\hline & $\mathrm{pBe} \rightarrow$ other $\rightarrow(\ldots) \rightarrow \bar{\nu}_{\mu}$ & $0.5 \%$ \\
\hline \multirow{5}{*}{$\bar{\nu}_{e}(0.1 \%)$} & $\mathrm{pBe} \rightarrow \pi^{ \pm} \rightarrow(\ldots) \rightarrow \bar{\nu}_{e}$ & $12.9 \%$ \\
\hline & $\mathrm{pBe} \rightarrow K^{ \pm} \rightarrow(\ldots) \rightarrow \bar{\nu}_{e}$ & $6.3 \%$ \\
\hline & $\mathrm{pBe} \rightarrow K_{L}^{0} \rightarrow(\ldots) \rightarrow \bar{\nu}_{e}$ & $65.2 \%$ \\
\hline & $\mathrm{pBe} \rightarrow \mathrm{p}$ or $\mathrm{n} \rightarrow(\ldots) \rightarrow \bar{\nu}_{e}$ & $15.3 \%$ \\
\hline & $\mathrm{pBe} \rightarrow$ other $\rightarrow(\ldots) \rightarrow \bar{\nu}_{e}$ & $0.3 \%$ \\
\hline
\end{tabular}


where $\sigma_{\mathrm{ELA}}$ is the total elastic cross section and $\sigma_{\mathrm{PROD}}$ is the cross section for particle production processes described earlier. The parameterizations of the total cross sections for $p$ or $n$, used in [144] are of the form:

$$
\sigma=A+B \times p^{n}+C \times \ln ^{2} p+D \times \ln p
$$

where A,B,C,D, and $n$ are constants to be fit, and $p$ is the particle's momentum. For $\pi^{ \pm}$-Nucleus cross sections a slightly more complicated form was used to take into account the occurrence of resonances:

$$
\begin{array}{r}
\sigma=\left[1+\tanh \left\{\theta_{s}\left(p-\theta_{0}\right)\right\}\right] \times\left[A+B p^{n}+C \times \ln ^{2} p\right] \\
+N_{R}\left|\frac{-m(p) \Gamma_{R}}{M_{R}^{2}-m(p)^{2}+i m(p) \Gamma_{R}}\right|^{2}
\end{array}
$$

where a threshold shape in the form of a hyperbolic tangent suppresses the low momentum contribution so that the Breit-Wigner form dominates in the presence of a resonance with width $\Gamma_{R}$. The parameterizations and the models used to predict the hadronic interaction cross sections are described in detail in [144, 145], and are shown in Figure 3.5 for $(p / n)$-Be interactions.

The $(p / n)$-Nucleus total cross sections are calculated using the Glauber model [146], which yields good agreement with published experimental measurements of the $n$-Be and $n$ - $\mathrm{Al}$ cross sections in the momentum range from 2.0-9.0 GeV/c. No data is available for $p$-Be cross sections, but isospin symmetry assures that it should be equally well described. A parameterization which describes the data and theoretical calculations well is used in this range, and is shown in Figure 3.5(a) for $(p / n)$-Be interactions.

The $\pi^{ \pm}$-Nucleus total cross section is parameterized by fitting Glauber model predictions at high momenta and existing experimental data at low momenta obtaining a model in the full range $0.5-6.0 \mathrm{GeV} / c$. This is shown in Figure 3.5(b) for $\pi^{+}$-Be interactions. 
The $(p / n)$-Nucleus and $\pi^{ \pm}$-Nucleus inelastic cross sections are derived entirely from existing measurements and fit to a parameterization in the range 2.0-9.0 GeV/c and 0.5-6.0 GeV/c respectively. These are shown in Figures 3.5(c) and 3.5(d) for $(p / n)$-Be and $\pi^{+}$-Be interactions respectively.

There are no measurements of the $(p / n)$-Nucleus Quasi-Elastic cross section. These are instead theoretically calculated accounting for shadowing effects and the values are parameterized in the interval from $2.0-9.0 \mathrm{GeV} / c$. The parameterization is checked against the prediction of other hadronic interaction packages like FLUKA [147] and found to be in good agreement. This is shown in Figure 3.5(e) for $(p / n)$-Be interactions.

Similarly, there are limited measurements of the $\pi^{ \pm}$-Nucleus Quasi-Elastic cross section around the $\Delta$ resonance, therefore the theoretically calculated values and the available data are parameterized in the momentum range of $0.5-6.0 \mathrm{GeV} / c$. Figure 3.5(e) for $\pi^{+}$-Be interactions.

\subsubsection{Systematic uncertainties in the flux prediction}

In addition to the uncertainties encoded in the covariance matrices extracted from the fits to hadron production data with Sanford-Wang and Feynman-Scaling parameterizations (Tables 3.2, 3.3, 3.4, and 3.5), we consider the uncertainty associated with the propagation of the secondary particles across the target and horn materials (Beryllium and Aluminum respectively) inherent in the modeling of hadronic interactions.

The parameterizations used to describe the hadronic interaction data and models provide a way to define systematic uncertainty bands that cover the variations in the data or the models. Systematic excursions of the flux prediction were estimated to 
represent one standard deviations covering the spread of available data and theoretical uncertainties. The effect of these excursions on the flux was calculated by running the beam Monte Carlo simulation for each one of them.

Also considered are excursions for the skin depth in the model of the horn inner conductor and the intensity of the current flowing through it were made. The magnitude of the changes caused by these excursions on the total neutrino flux is shown in Table 3.7.

The sources of systematic uncertainty of secondaries as they interact within the target and the horn materials are listed in Table 3.7.

Table 3.7: Sources of systematic uncertainty associated with the hadronic interactions models at the target, and the horn focusing

\begin{tabular}{l|c}
\hline \hline Source of Uncertainty & Percent Change in Total Neutrino Flux \\
\hline Skin Depth & $2.89 \%$ \\
Horn Current & $0.31 \%$ \\
\hline$(\mathrm{p} / \mathrm{n})$-Be $\sigma_{\text {inel }}$ & $0.09 \%$ \\
$(\mathrm{p} / \mathrm{n})-\mathrm{Be} \sigma_{\mathrm{QE}}$ & $3.03 \%$ \\
$(\mathrm{p} / \mathrm{n})-\mathrm{Be} \sigma_{\text {total }}$ & $0.27 \%$ \\
\hline$\pi$-Be $\sigma_{\text {inel }}$ & $0.01 \%$ \\
$\pi$-Be $\sigma_{\mathrm{QE}}$ & $0.86 \%$ \\
$\pi$-Be $\sigma_{\text {total }}$ & $0.32 \%$ \\
\hline \hline Total & $4.31 \%$ \\
\hline
\end{tabular}




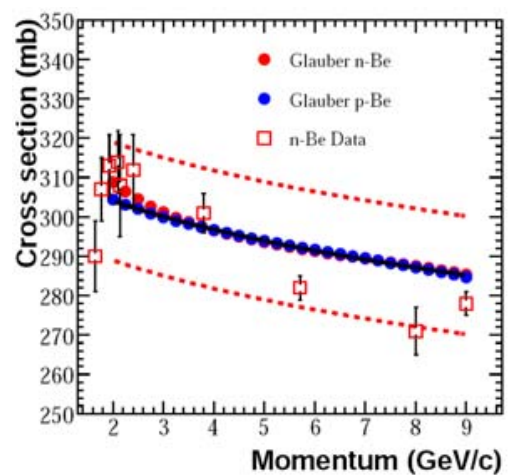

(a) $(\mathrm{p} / \mathrm{n})-\mathrm{Be}$, Total.

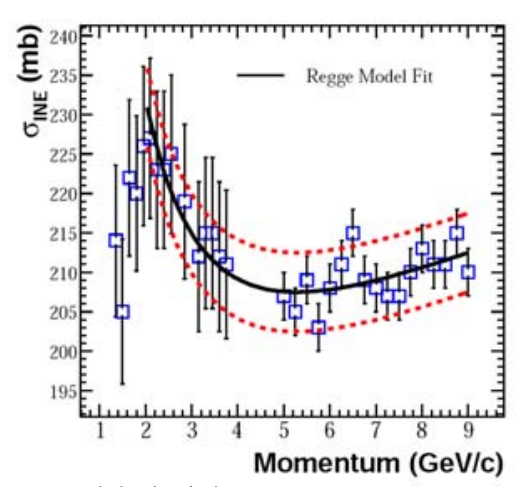

(c) $(\mathrm{p} / \mathrm{n})$-Be inelastic.

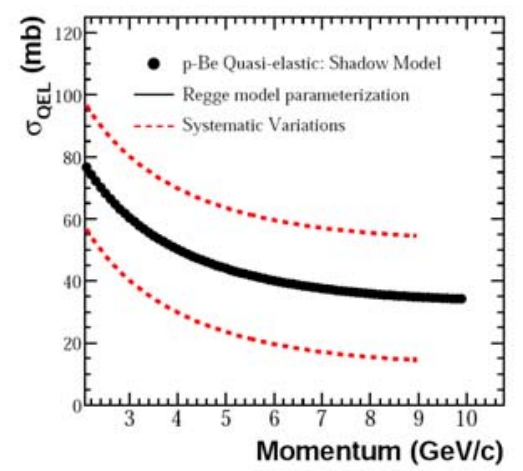

(e) $(\mathrm{p} / \mathrm{n})$-Be Quasi-Elastic

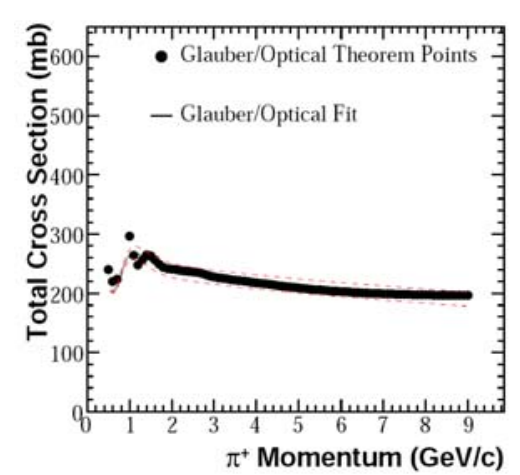

(b) $\pi^{+}$-Be total.

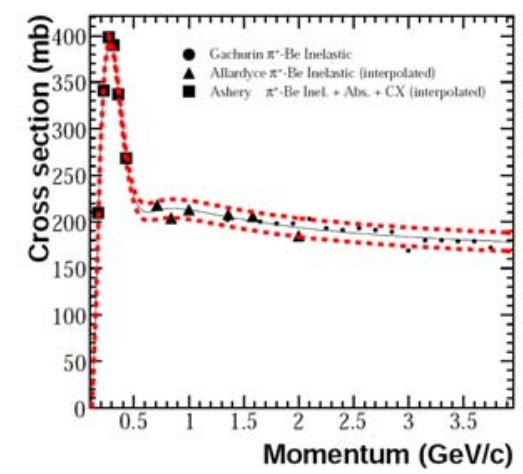

(d) $\pi^{+}$-Be inelastic.

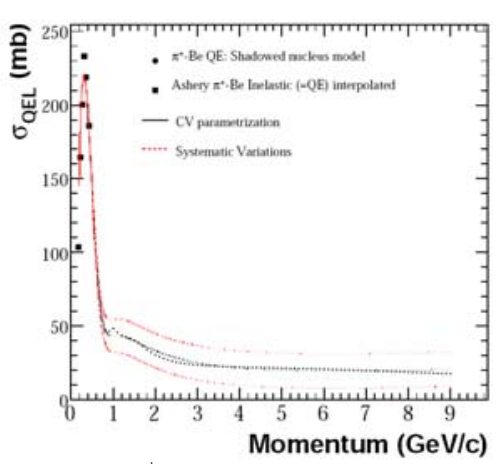

(f) $\pi^{+}$-Be Quasi-Elastic

Figure 3.5: The total (a-b), inelastic (c-d) and quasi-elastic (d-e) cross sections for $p / n$ and $\pi^{+}$interactions in Beryllium in the MiniBooNE beam Monte Carlo simulation. The error bands shown are calculated by varying the coefficients in the parameterizations to cover the spread in the data and theoretical uncertainties. Taken from [145]. 


\subsection{NUANCE cross section generator}

The experiment uses the NUANCE [148] event generator to simulate neutrino interactions in the detector volume.

At high energies neutrino cross sections are well known (to within $\sim 1 \%-2 \%$ ), however, in the few-GeV range experimental and theoretical uncertainties range between $\sim 10 \%$ to $20 \%$. Figure 3.6 shows the available experimental constraints on deep inelastic scattering (DIS), quasi-elastic (QE), and single pion ( $1 \pi)$ cross sections in the range from 0 to $300 \mathrm{GeV}$. Most of the existing measurements were done using light targets. The bulk of the interactions in MiniBooNE occur in nucleons bound in ${ }^{12} \mathrm{C}$ nuclei, therefore nuclear effects must be modeled to incorporate the knowledge of cross sections on free nucleons obtained from measurements on light targets.

\subsubsection{The CCQE interaction}

The dominant channel for a neutrino to interact with a nucleon in the energy range between 200 and $2000 \mathrm{MeV}$ is the charged current quasi-elastic (CCQE) interaction:

$$
\begin{aligned}
& \nu_{\ell}+n \rightarrow \ell^{-}+p \\
& \bar{\nu}_{\ell}+p \rightarrow \ell^{+}+n, \quad \ell=e, \mu .
\end{aligned}
$$

This process, shown in Figure 3.7 is an ideal signal in a neutrino oscillations search because it has a large cross section and identifies the flavor of the incident neutrino. The CCQE cross section is given by [185]:

$$
\begin{aligned}
& \frac{d \sigma}{d q^{2}}=\frac{M^{2} G_{F}\left|V_{u d}\right|^{2}}{8 \pi E_{\nu}^{2}}\left[A\left(q^{2}\right) \mp B\left(q^{2}\right) \frac{s-u}{M^{2}}+C\left(q^{2}\right) \frac{(s-u)^{2}}{M^{4}}\right] \\
& (s-u)^{2}=4 M E-q^{2}-m_{\ell}^{2},
\end{aligned}
$$

where the $(+)-\operatorname{sign}$ refers to (anti)neutrino scattering, $M=\frac{1}{2}\left(M_{p}+M_{n}\right)$ is the average of the proton and neutron masses, $q=\left(p_{\nu}-p_{\ell}\right)=\left(p_{N}-p_{N}^{\prime}\right)$ is the four- 


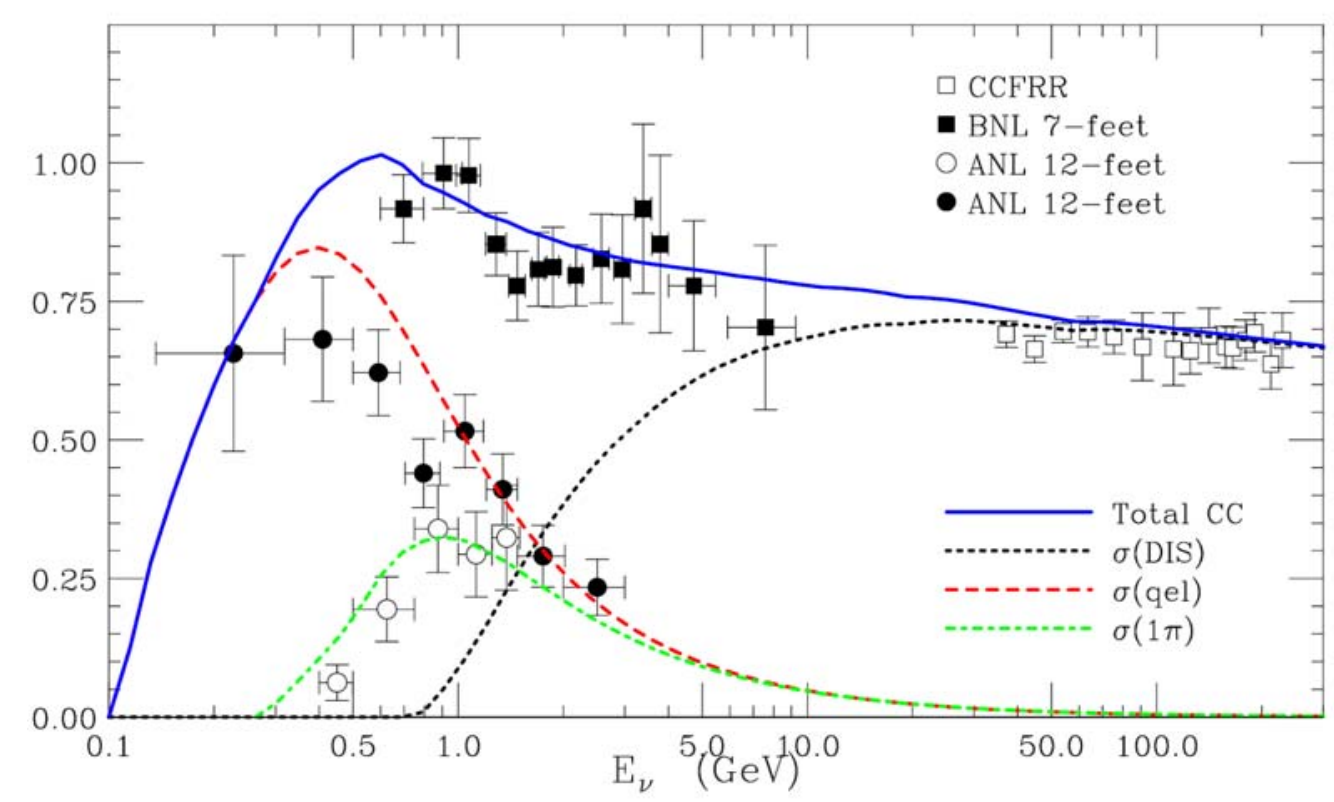

Figure 3.6: Total $\nu_{\mu}$ charged-current cross section per nucleon divided by neutrino energy, $\sigma_{C C} / E_{\nu}$ (blue curve), in the energy range $0.1<E_{\nu}(G e V)<300$. Also shown are the predicted CCQE (red), CC resonant single $\pi$ production (green), and CC DIS (black) contributions [120]. 


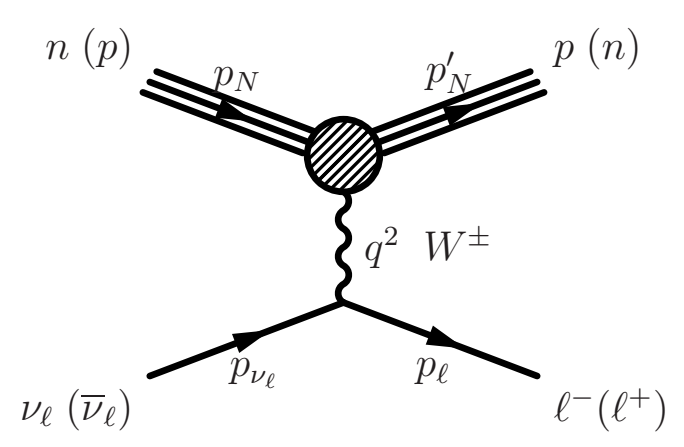

Figure 3.7: Feynman diagram for the CCQE process on a free nucleon.

momentum transfer, $s=\left(p_{\nu}+p_{N}\right)$ and $u=\left(p_{\nu}-p_{N}^{\prime}\right)$ are the usual Mandelstam variables, $V_{u d}$ is the CKM matrix element connecting the $u$ and $d$ quarks, and $G_{F}=$ $1.1803 \times 10^{-5} \mathrm{GeV}^{-2}$ is the Fermi constant. The functions $A\left(q^{2}\right), B\left(q^{2}\right)$, and $C\left(q^{2}\right)$ contain the information about the structure of the nucleons and can be written in terms of the four real form factors $F_{V}^{1}\left(q^{2}\right)$ (isovector Dirac), $F_{V}^{2}\left(q^{2}\right)$ (isovector Pauli), $F_{A}\left(q^{2}\right)$ (axial-vector), and $F_{P}\left(q^{2}\right)$ (induced pseudoscalar) as follows [185] :

$$
\begin{aligned}
A\left(q^{2}\right)= & \frac{m_{\ell}^{2}-q^{2}}{4 M^{2}}\left\{\left(4-\frac{q^{2}}{M^{2}}\right)\left(F_{A}\right)^{2}-\left(4+\frac{q^{2}}{M^{2}}\right)\left(F_{1 V}\right)^{2}-\frac{q^{2}}{M^{2}}\left(\xi F_{2 V}\right)^{2}\left(1+\frac{q^{2}}{4 M^{2}}\right)\right. \\
& \left.-\frac{4 q^{2}}{M^{2}}\left(F_{V}^{1} \xi F_{V}^{2}\right)-\frac{m_{\ell}^{2}}{M^{2}}\left[\left(F_{V}^{1}+\xi F_{V}^{2}\right)^{2}+\left(F_{A}+2 F_{P}\right)^{2}+\left(\frac{q^{2}}{M^{2}}-4\right)\right]\left(F_{P}\right)^{2}\right\} \\
B\left(q^{2}\right)= & -\frac{q^{2}}{M^{2}}\left[F_{A}\left(F_{V}^{1}+\xi F_{V}^{2}\right)\right] \\
C\left(q^{2}\right)= & \frac{1}{4}\left(\left(F_{A}\right)^{2}+\left(F_{V}^{1}\right)^{2}-\frac{q^{2}}{M^{2}}\left(\frac{1}{2} \xi F_{V}^{2}\right)^{2}\right),
\end{aligned}
$$

where $\xi=\left(\mu_{p}-\mu_{n}\right) / \mu_{N}=3.706$ is the difference between the anomalous magnetic moment of the proton and the neutron expressed in units of the Nuclear Magneton $\mu_{N}$. The isovector Dirac and Pauli form factors $F_{V}^{1}$ and $F_{V}^{2}$ are related to the isovector electric and magnetic form factors (Sachs form factors) $G_{E}^{V}$ and $G_{M}^{V}$ obtained from electron scattering experiments by:

$$
\begin{aligned}
& G_{E}^{V}\left(q^{2}\right)=F_{V}^{1}\left(q^{2}\right)+\frac{q^{2}}{4 M^{2}} \xi F_{V}^{2}\left(q^{2}\right) \\
& G_{M}^{V}\left(q^{2}\right)=F_{V}^{1}\left(q^{2}\right)+\xi F_{V}^{2}\left(q^{2}\right)
\end{aligned}
$$


These are described to $\sim 5 \%$ accuracy [187] by the dipole form

$$
\frac{G_{E}^{V}\left(q^{2}\right)}{G_{E}^{V}(0)}=\frac{G_{M}^{V}\left(q^{2}\right)}{G_{M}^{V}(0)}\left(1-\frac{q^{2}}{M_{V}^{2}}\right)^{-2}
$$

The parameter $M_{V}$ is called the vector mass, and it is experimentally found to be $M_{V} \approx 0.84 \mathrm{GeV}$. Moreover, the electric and magnetic form factors satisfy the scaling relation $G_{E}^{V}\left(q^{2}\right)=(1-\xi)^{-1} G_{M}^{V}\left(q^{2}\right)$.

The axial-vector form factor $F_{A}$ is presumed to also obey the dipole form as well, with a parameter called the axial mass $M_{A}$ :

$$
F_{A}\left(q^{2}\right)=F_{A}(0)\left(1-\frac{q^{2}}{M_{A}^{2}}\right)^{-2},
$$

where $F_{A}(0)=-1.2674 \pm 0.0035$, and the world average value of the axial mass from neutrino experiments is $M_{A}=1.026 \pm 0.021 \mathrm{GeV}$ [188].

Finally, the pseudo-scalar form factor $F_{P}$ is [189]

$$
F_{P}\left(q^{2}\right)=F_{A}\left(q^{2}\right) \frac{2 M}{m_{\pi}^{2}-q^{2}}
$$

with $m_{\pi}$ the pion mass. Details on the determination of the $q^{2}$ dependence of the various form factors can be found elsewhere [22] [24] [185] [187] [189]. 


\section{Nuclear effects in CCQE scattering}

The majority of the nucleons with which neutrinos interact in MiniBooNE are bound in the interior of ${ }^{12} \mathrm{C}$ nuclei. To introduce this effect in the determination of the CCQE cross sections, NUANCE uses the formalism of Smith and Moniz [186]. Some details of this formalism are necessary to understand the evaluation of the systematic uncertainties in the CCQE cross section used in this thesis.

Following Ref.[186] we express the cross section for CCQE scattering off an entire nucleus in terms of a leptonic tensor $\eta_{\mu \nu}$ and a nuclear tensor $W_{\nu \mu}$ :

$$
\begin{aligned}
\frac{d^{3} \sigma}{d^{3} p_{\ell}} & =\frac{G_{F}^{2}}{2} \frac{1}{(2 \pi)^{2}} \frac{1}{2\left|p_{\nu} \cdot p_{N}\right|} \frac{1}{2 E_{\ell}} \eta_{\nu \mu} W_{\nu \mu} \\
\eta_{\nu \mu} & =\operatorname{Tr}\left\{\gamma_{\nu}\left(1+\gamma_{5}\right) p_{\nu} \gamma_{\mu}\left(1+\gamma_{5}\right) p_{\ell}\right\} \\
W_{\mu \nu} & =(2 \pi)^{3} \Omega \sum_{i} \sum_{f} \delta^{(4)}\left(p_{N}-p_{N}^{\prime}-q\right)\left\langle n\left(p_{N}\right)\left|J_{\mu}^{+}\right| p\left(p_{N}^{\prime}\right)\right\rangle\left\langle n\left(p_{N}^{\prime}\right)\left|J_{\mu}^{-}\right| n\left(p_{N}\right)\right\rangle E_{T}
\end{aligned}
$$

where $J^{+}\left(J^{-}\right)$is the nuclear current connecting the initial (final) to the final (initial) state nuclei, $E_{T}$ is the energy of the target nucleus, and the sums indicate averaging over initial and final polarization states.

The target nucleus state is described as a superposition of two non-interacting Fermi gases of neutrons and protons with momentum distributions $n_{n}(k)=n_{p}(k)=$ $\theta\left(k_{F}-|\mathbf{k}|\right)$. For a simple Fermi gas the quantization volume $\Omega$ can be replaced by $\Omega=\frac{3 \pi^{2} N}{k_{F_{n}}^{3}}$, with $N$ the number of neutrons in the nucleus and $k_{F}$ is the Fermi momentum of the nucleon gas system.

Summing over the contribution of all the nucleons below the Fermi surface the tensor $W_{\mu \nu}$ can be calculated in terms of a single particle tensor $T_{\mu \nu}$ involving the single nucleon transition current $j^{+}\left(j^{-}\right)$connecting a neutron (proton) to a proton (neutron), and the energy transfered to the nucleus $\omega=q^{0}$ :

$$
W_{\mu \nu}=\int_{E_{l o}}^{E_{h i}} d^{3} k f(\mathbf{k}, \mathbf{q}, \omega) T_{\mu \nu} .
$$


where $f(\mathbf{k}, \mathbf{q}, \omega)$ is the nucleon phase space density function. If $E_{B}$ is the binding energy per nucleon, there is an effective energy transfer $\omega_{\text {eff }}=\omega-E_{B}$, and an effective four-momentum transfer $q_{\text {eff }}^{2}=q^{2}-\omega_{\text {eff }}^{2}-M_{T}^{\prime 2}-M_{T}^{2}$, where $M_{T}^{\prime}$ is the mass of the recoil nucleus. The limits of integration are:

$$
\begin{aligned}
& E_{h i}=\sqrt{k_{F}^{2}+M^{2}} \quad \text { The Fermi energy, } \\
& E_{l o}=\max \left\{E_{h i}-w_{\text {eff }}, M\left(\frac{x y \sqrt{1-x^{2}+y^{2}}}{1-y^{2}}\right)\right\}, x=\left(\frac{q_{e f f}^{2}}{2 M q}\right), y=\left(\frac{-\omega_{e f f}}{q}\right) .
\end{aligned}
$$

Reference [186] writes $f(\mathbf{k}, \mathbf{q}, \omega)$ as:

$$
\begin{aligned}
f(\mathbf{k}, \mathbf{q}, \omega) & =\frac{M \Omega}{\left(2 \pi^{3}\right)} \frac{n_{i}(k)\left[1-n_{f}(|\mathbf{k}-\mathbf{q}|)\right]}{E_{k} E_{k-q}} \delta^{(4)}\left(E_{k}-E_{k-q}+\omega\right) \\
T_{\mu \nu} & =E_{k} E_{k-q} \Omega^{2} \sum_{\lambda \lambda^{\prime}}\left\langle\mathbf{k}-\mathbf{q}, \lambda^{\prime}\left|j_{\mu}^{+}(0)\right| \mathbf{k}, \lambda\right\rangle\left\langle\mathbf{k}, \lambda\left|j_{\nu}^{-}(0)\right| \mathbf{k}-\mathbf{q}, \lambda^{\prime}\right\rangle
\end{aligned}
$$

The Pauli exclusion factor $\left[1-n_{f}(|\mathbf{k}-\mathbf{q}|)\right]$ guarantees that the recoil nucleon is outside the Fermi sphere, otherwise the interaction is Pauli-blocked.

The single particle nucleon tensor $T_{\mu \nu}$ can be written in terms of free nucleon form factors:

$$
\begin{aligned}
T_{\mu \nu}=T_{1} \delta_{\mu \nu}+ & T_{2} / M^{2} k_{\mu} k_{\nu} \quad+T_{\alpha} / M^{2} q_{\mu} q_{\nu} \\
& +T_{\beta} / M^{2}\left(k_{\mu} q_{\nu}+k_{\nu} q_{\mu}\right) \pm T_{8} / M^{2} \epsilon_{\mu \nu \sigma \tau} k_{\sigma} q_{\tau}
\end{aligned}
$$

which reference [186] calculates in terms of the free nucleon form factors of LlewellynSmith [185]:

$$
\begin{aligned}
& T_{1}=\frac{1}{2} q^{2}\left(F_{V}^{1}+2 M \xi F_{V}^{2}\right)^{2}+\left(2 M^{2}+\frac{1}{2} q^{2}\right)\left(F_{A}\right)^{2} \\
& T_{2}=2 M^{2}\left(\left(F_{V}^{1}\right)^{2}+q^{2}\left(\xi F_{V}^{2}\right)^{2}+\left(F_{A}\right)^{2}\right) \\
& T_{\alpha}=-\frac{M^{2}}{q^{2}} T_{1}+\frac{1}{4} T_{2}+\frac{M^{2}}{2 q^{2}}\left(2 M F_{A}-q^{2} F_{P}\right)^{2} \\
& T_{\beta}=-\frac{1}{2} T_{2}, \quad T_{8}=2 M^{2} F_{A}\left(F_{V}^{1}+2 M \xi F_{V}^{2}\right)
\end{aligned}
$$

Analogous form factors can be defined for the nuclear tensor $W_{\nu \mu}$ :

$$
\begin{aligned}
W_{\mu \nu}=W_{1} \delta_{\mu \nu} & +W_{2} / M_{T}^{2} p_{\mu} p_{\nu}+W_{\alpha} / M_{T}^{2} q_{\mu} q_{\nu} \\
& +W_{\beta} / M_{T}^{2}\left(p_{\mu} q_{\nu}+p_{\nu} q_{\mu}\right) \pm W_{8} / M_{T}^{2} \epsilon_{\mu \nu \sigma \tau} p_{\sigma} q_{\tau}
\end{aligned}
$$


where now the mass of the target nucleus $M_{T}$ is used instead. The relation between the nuclear and free-nucleon form factors can be found analytically for a Fermi gas [186]:

$$
\begin{aligned}
& W_{1}=a_{1} T_{1}+\frac{1}{2}\left(a_{2}-a_{3}\right) T_{2} \\
& W_{2}=\left[a_{4}+\frac{2 \omega}{|\mathbf{q}|} a_{5}+\frac{\omega^{2}}{|\mathbf{q}|^{2}} a_{3}+\frac{q^{2}}{2|\mathbf{q}|^{2}}\left(a_{2}-a_{3}\right)\right] T_{2} \\
& W_{\alpha}=\left(\frac{3}{2} a_{3}-\frac{1}{2} a_{2}\right) \frac{M_{T}^{2}}{|\mathbf{q}|^{2}} T_{2}+\frac{M_{T}^{2}}{M^{\prime 2}} a_{1} T_{\alpha}+\frac{2 M_{T}^{2}}{|\mathbf{q}| M^{\prime}} a_{6} T_{\beta} \\
& W_{\beta}=\frac{M_{T}}{M^{\prime}}\left(a_{7}+\frac{\omega}{|\mathbf{q}|} a_{6}\right) T_{\beta}+\frac{M_{T}}{|\mathbf{q}|}\left(a_{5}+\frac{\omega}{|\mathbf{q}|}\left(\frac{3}{2} a_{3}-\frac{1}{2} a_{2}\right)\right) T_{2} \\
& W_{8}=\frac{M_{T}}{M^{\prime}}\left(a_{7}+\frac{\omega}{|\mathbf{q}|} a_{6}\right) T_{8},
\end{aligned}
$$

where using the quantities defined in $\mathrm{Eq}(3.12)$, the constants $a_{i}$ are given by:

$$
\begin{aligned}
& a_{1}=b_{0}, \quad a_{2}=b_{2}-b_{0}, \quad a_{3}=y^{2} b_{2}+2 x y b_{1}+x^{2} b_{0}, \\
& a_{4}=b_{2}-\frac{2 E_{B}}{M^{\prime}} b_{1}+\left(\frac{E_{B}}{M^{\prime}}\right)^{2} b_{0}, \quad a_{5}=y b_{2}+\left(x-\frac{E_{B}}{M^{\prime}} y\right) b_{1}-\frac{E_{B}}{M^{\prime}} x b_{0} \\
& a_{6}=x b_{0}+y b_{1}, \\
& a_{7}=b_{1}-\frac{E_{B}}{M^{\prime}} b_{0}, \\
& b_{n}=\frac{M_{T} \Omega}{(2 \pi)^{2}|\mathbf{q}|}\left(\frac{1}{M^{\prime}}\right)^{n}\left\{\sum_{k=1}^{n+1} \frac{1}{k}\left(E_{h i}-E_{l o}\right) E^{n-k+1}+E_{B}^{n+1} \ln \left(\frac{E_{h i}-E_{B}}{E_{l o}-E_{B}}\right)\right\}
\end{aligned}
$$

The CCQE cross section on the nuclear system in the laboratory frame used by the NUANCE cross section generator can be found by substituting the above expressions for the tensor $W_{\mu \nu}$ into the following formula taken from reference [186]:

$$
\begin{aligned}
\frac{d^{2} \sigma}{d p_{\ell} d \Omega} & =\frac{\left(V_{u d} G_{F}\right)^{2} p_{\ell}^{2} \cos ^{2}\left(\frac{1}{2} \chi\right)}{2 \pi^{2} M}\left(W_{2}+\left[2 W_{1}+\frac{m_{\ell}^{2}}{M^{2}} W_{\alpha}\right] \tan ^{2}\left(\frac{1}{2} \chi\right)\right. \\
& +\left(W_{\beta} \pm W_{8}\right) m_{\ell}^{2} /\left(M E_{\ell} \cos ^{2}\left(\frac{1}{2} \chi\right)\right) \\
& \left.-2 W_{8} / M \tan \left(\frac{1}{2} \chi\right) \sec \left(\frac{1}{2} \chi\right)\left[q^{2} \cos ^{2}\left(\frac{1}{2} \chi\right)+|\mathbf{q}|^{2} \cos ^{2}\left(\frac{1}{2} \chi\right)+m_{\ell}^{2}\right]^{\frac{1}{2}}\right)
\end{aligned}
$$

where $\cos \chi=p_{\ell} / E_{\ell} \cos \theta_{\ell}$, with $\theta_{\ell}$ the scattering angle of the lepton in the laboratory frame. The $+(-)$ sign of $W_{8}$ corresponds to neutrino (anti-neutrino) scattering. 


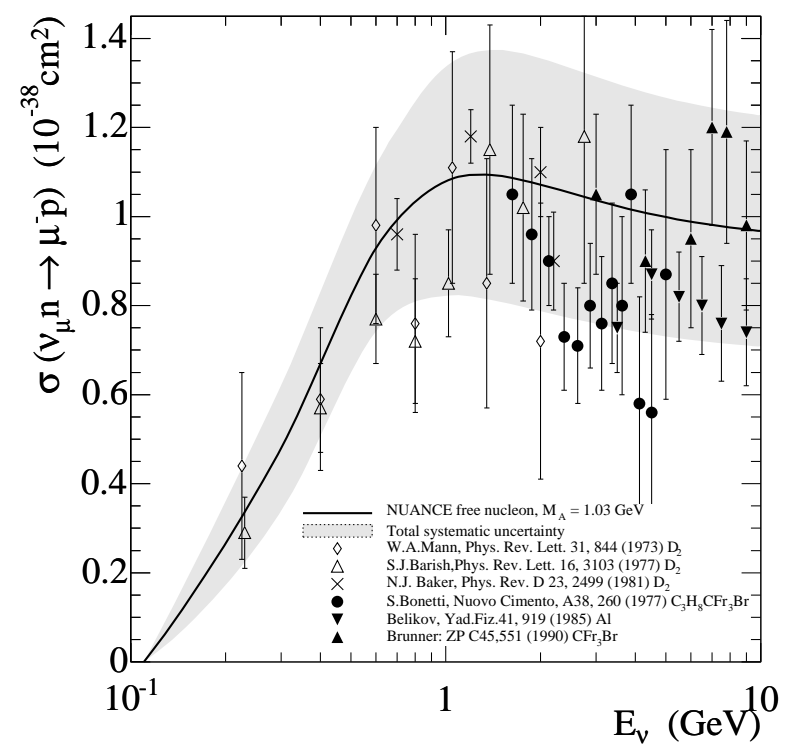

Figure 3.8: NUANCE prediction for the $\nu_{\mu}$-CCQE cross section compared to experimental data on light $\left(\mathrm{D}_{2}\right)$ and heavy (typically ${ }^{12} \mathrm{C}$ ) nuclear targets. The solid curve and light colored band show, respectively, the cross-section on free nucleons with $M_{A}=1.03 \mathrm{GeV}$ and its estimated uncertainty.

\section{CCQE kinematics}

The energy of the interacting neutrino can be calculated from the kinematics of the final state if the scattering angle and energy of both outgoing particles is determined. In general, as is the case for MiniBooNE, the track of the outgoing nucleon is hard to measure, but the outgoing lepton energy and scattering angle are well determined. In this case the neutrino energy can be approximated by assuming that the incident nucleon is at rest.

In a quasi-elastic interaction with a free neutron at rest like that shown in Figure 3.9 the energy of the incoming neutrino is completely determined by the energy of the outgoing lepton $E_{\ell}$, and its scattering angle with respect to the incident neutrino 

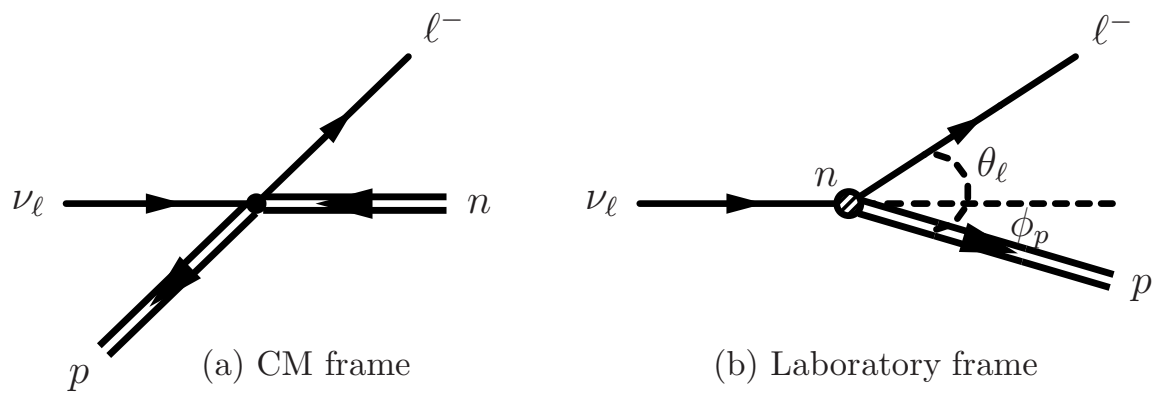

(b) Laboratory frame

Figure 3.9: CCQE neutrino scattering on a neutron at rest in (a) the center of mass frame, and (b) the laboratory frame. In the laboratory frame, the outgoing lepton (proton) makes an angle $\theta_{\ell}\left(\phi_{p}\right)$ with the original neutrino direction.

direction $\theta_{\ell}$ :

$$
E_{\nu}^{Q E}\left(\mathbf{p}_{n}=0\right)=\frac{1}{2} \frac{2 M E_{\ell}-m_{\ell}^{2}}{M-E_{\ell}+\sqrt{E_{\ell}^{2}-m_{\ell}^{2}} \cos \theta_{\ell}}
$$

The four momentum transfer $Q^{2}=-q^{2}=-\left(p_{\nu}-p_{\ell}\right)^{2}$ is also given in terms of these quantities:

$$
Q^{2}=2 E_{\nu} E_{\ell}\left(1-\beta_{\ell} \cos \theta_{\ell}\right)-m_{\ell}^{2}
$$

where $\beta_{\ell}$ is the velocity of the outgoing lepton, but this expression is general and does not assume anything for the target neutron. The Fermi gas model in the NUANCE simulation has the neutrons in a potential well with binding energy $B=34 \mathrm{MeV}$ and satisfying the dispersion relation $\left(E_{n}-B\right)^{2}=\left|\mathbf{p}_{n}\right|^{2}-M_{n}^{2}$. The maximum momentum of a neutron inside the bound nucleus (the Fermi Momentum) is set to $p_{F}=220$ $\mathrm{MeV} / c$. The binding energy and Fermi momentum are set by electron scattering data. A calibration procedure aimed at reducing the effect of ignoring Fermi motion in the calculation of the neutrino energy in a CCQE interaction (for both, $\nu_{e}$ and $\nu_{\mu}$ ) was implemented and is described in Appendix A. This calibration procedure improves the energy resolution at $1 \mathrm{GeV}$ of muon energy from $\sim 15 \%$ to $\sim 10 \%$ by reducing the non-Gaussian tails in the distribution of fractional residuals $\left(E_{\nu}^{\text {rec }}-E_{\nu}^{\text {true }}\right) / E_{\nu}^{\text {true }}$. 


\subsubsection{Neutral Current $\pi^{0}$ production}

The largest $\nu_{\mu}$-induced background to the MiniBooNE $\nu_{e}$ appearance search is the production of $\pi^{0}$ 's via neutral current interactions. The dominant mode of NC $\pi^{0}$ production is the excitation of nucleons into baryonic resonances like the $\Delta(1232)$ and their subsequent decay, a process illustrated in Figure 3.10. NUANCE predictions of this process use the formalism of Rein and Sehgal [150] incorporating a total of sixteen higher mass resonances and their interference effects.

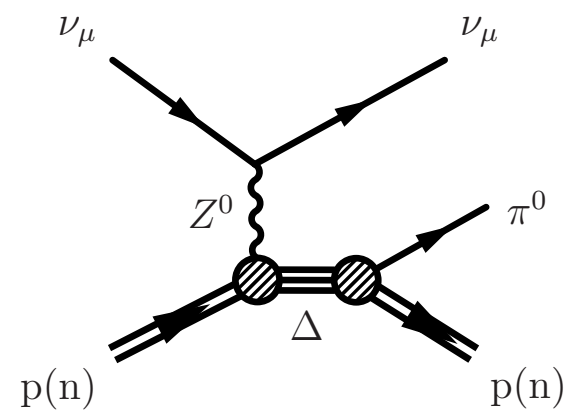

Figure 3.10: Neutral Current resonant $\pi^{0}$ production via the excitation of a nucleon into a $\Delta(1232)$ resonance.

Existing predictions of the cross sections for this process have large uncertainties, especially at the energies of interest to MiniBooNE. Only a handful of measurements have been performed to date, including a re-analysis of the Gargamelle bubble chamber data [151], a measurement from $\mathrm{K} 2 \mathrm{~K}$ at $1.3 \mathrm{GeV}$ of the ratio of $\mathrm{NC}$ to $\mathrm{CC} \pi^{0}$ cross section [152], and a measurement in an early spark chamber experiment [153].

Another NC process producing $\pi^{0}$ 's is the coherent scattering of a neutrino off a nucleus as a whole: $\nu_{\mu} A \rightarrow \nu_{\mu} A \pi^{0}$, which NUANCE also simulates using the formalism in [150]. Investigations of $\mathrm{NC} \pi^{0}$ production with the MiniBooNE detector [183] [165] suggest that coherent $\pi^{0}$ production is not negligible ${ }^{1}$.

\footnotetext{
${ }^{1}$ Recent measurements of $\mathrm{K} 2 \mathrm{~K}[163]$ suggest that the coherent production of $\pi^{0} \mathrm{~s}$ in $\mathrm{CC}$ interactions is negligible. The authors in [164] suggest that nuclear effects can account for the different behavior in $\mathrm{CC} \pi^{0}$ production in $\mathrm{K} 2 \mathrm{~K}$ and $\mathrm{NC} \pi^{0}$ production in MiniBooNE.
} 
For these reasons a direct measurement of the rate of $\mathrm{NC} \pi^{0}$ events in MiniBooNE is the most direct way to correct the predictions from the simulation and to constrain the uncertainties associated with it. The measurement (described in detail elsewhere [149]) consists of assembling a sample of $\mathrm{NC} \pi^{0}$ interactions with high purity $(96.5 \%)$ and determine the yield of these events in nine bins of reconstructed momentum of the $\pi^{0}$. A comparison of this distribution between data and the simulation defines a correction factor that can be applied in each reconstructed $\pi^{0}$ momentum bin to bring the simulated distribution agreement with that of the data. The events from the data and Monte Carlo samples are required to have no following muon decay electron, an electron/muon likelihood ratio which favors the electron hypothesis and an electron/pion likelihood which favors the $\pi^{0}$ hypothesis ${ }^{2}$. As a final requirement, the events must have a reconstructed $\pi^{0}$ mass between 80 and $200 \mathrm{MeV} / c^{2}$ (around the $\pi^{0}$ mass peak). The comparison between the uncorrected $\pi^{0}$ momentum distribution in data and Monte Carlo with and without the correction factors is shown in Figure 3.11. The correction factors in each of the nine reconstructed momentum bins and their uncertainties expressed as a $9 \times 9$ covariance matrix are shown in Table 3.2.2. Figure 3.12 shows that prior to tuning, there is an excess of $\pi^{0}$ events at lower momenta relative to the uncorrected NUANCE prediction, while the higher momenta are in agreement. Based on these distributions, the rate of $\pi^{0}$ production is corrected in order to bring them in agreement with the data.

\subsubsection{Cross sections systematic uncertainties}

Fits to the $Q^{2}$ distribution shape for a sample of $\nu_{\mu}$ CCQE events were done following the methodology in [191] yielding values and correlations for the parameter $M_{A}$ and a scale factor $\kappa$ multiplying the low energy limit $E_{l o}$ in Eq.(3.12). It is important to

\footnotetext{
${ }^{2}$ For a description of these likelihoods and ratios see chapter 3.4 and the references therein.
} 


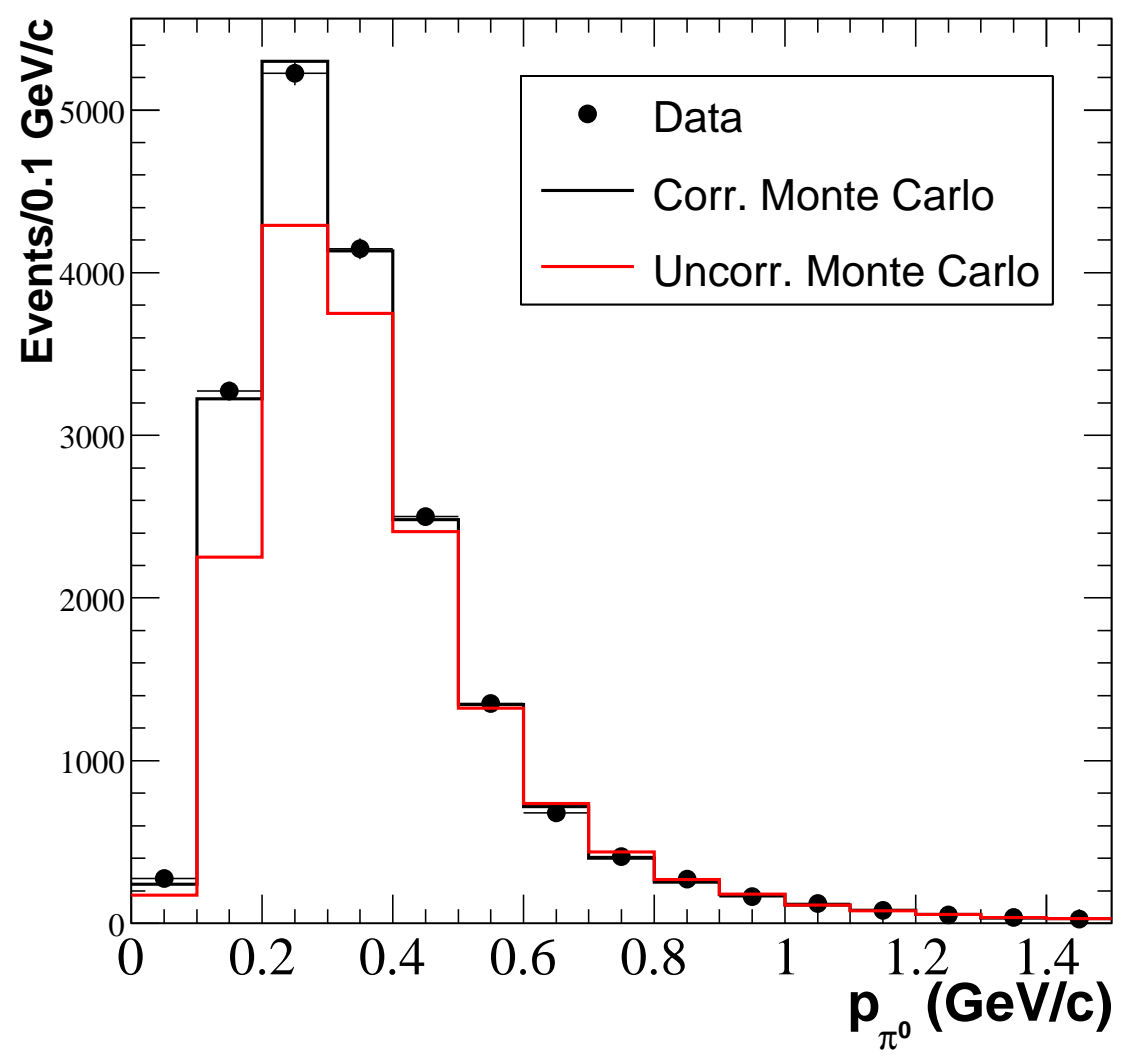

Figure 3.11: Reconstructed momentum distribution of $\pi^{0}$ events in data (points) and Monte Carlo (black histogram) following the application of correction factors in Table 3.2.2. The red histogram shows the uncorrected (default NUANCE) prediction. See text for more details. Taken from [149]. 

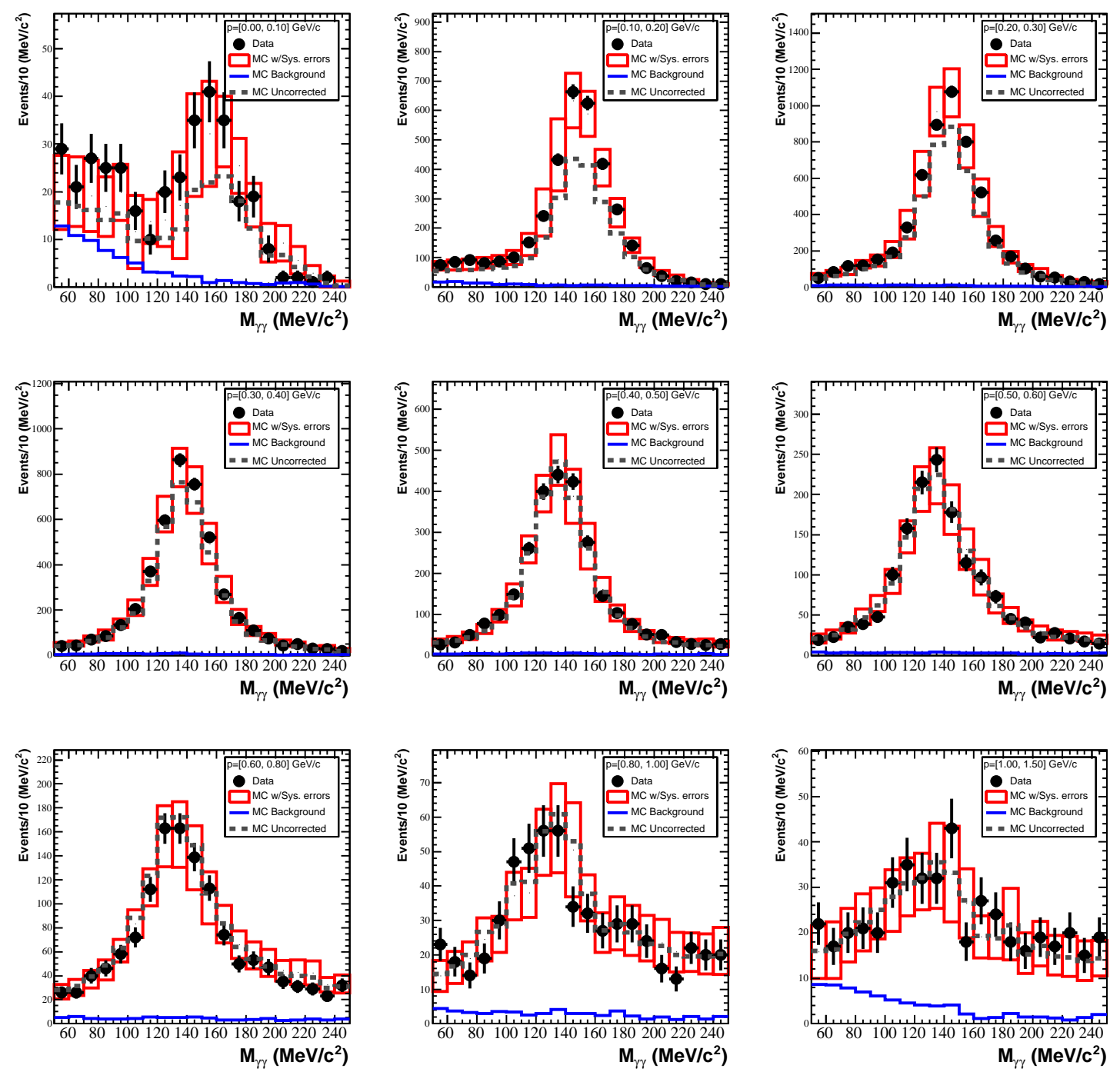

Figure 3.12: Reconstructed mass distribution of $\mathrm{NC} \pi^{0}$ events in reconstructed $\pi^{0}$ momentum bins. Systematic uncertainties are shown as red boxes whose centers mark the corrected predictions. The dashed histogram shows the uncorrected prediction from the NUANCE event generator. The blue solid histograms show the non- $\pi^{0}$ contamination of the total sample (as predicted after the correction). Taken from [149]. 
Table 3.8: The central values (CV) and covariance matrix for the correction factors applied to the NUANCE predicted yield of $\mathrm{NC} \pi^{0}$ events in the MiniBooNE detector in each of nine reconstructed $\pi^{0}$ momentum bins, as calculated by Djurcic et al. [132].

\begin{tabular}{c|ccccccccc}
\hline $\mathrm{CV}$ & 1.4477 & 1.4794 & 1.1301 & 1.0414 & 0.9515 & 1.0241 & 0.7071 & 0.9638 & 0.9684 \\
\hline \hline $\mathcal{M}_{i j}^{\pi^{0}}$ & bin 1 & bin 2 & bin 3 & bin 4 & bin 5 & bin 6 & bin 7 & bin 8 & bin 9 \\
\hline bin 1 & 0.1203 & 0.0401 & 0.0015 & -0.0079 & -0.0182 & -0.0455 & -0.0345 & -0.0672 & -0.0898 \\
bin 2 & 0.0401 & 0.0195 & 0.0008 & -0.0027 & -0.0074 & -0.0165 & -0.0161 & -0.0240 & -0.0392 \\
bin 3 & 0.0015 & 0.0008 & 0.0016 & 0.0001 & 0.0001 & -0.0003 & -0.0010 & -0.0024 & -0.0024 \\
bin 4 & -0.0079 & -0.0027 & 0.0001 & 0.0019 & 0.0009 & 0.0037 & 0.0024 & 0.0035 & 0.0063 \\
bin 5 & -0.0182 & -0.0074 & 0.0001 & 0.0009 & 0.0054 & 0.0043 & 0.0076 & 0.0081 & 0.0170 \\
bin 6 & -0.0455 & -0.0165 & -0.0003 & 0.0037 & 0.0043 & 0.0314 & 0.0104 & 0.0317 & 0.0412 \\
bin 7 & -0.0345 & -0.0161 & -0.0010 & 0.0024 & 0.0076 & 0.0104 & 0.0198 & 0.0203 & 0.0400 \\
bin 8 & -0.0672 & -0.0240 & -0.0024 & 0.0035 & 0.0081 & 0.0317 & 0.0203 & 0.0777 & 0.0540 \\
bin 9 & -0.0898 & -0.0392 & -0.0024 & 0.0063 & 0.0170 & 0.0412 & 0.0400 & 0.0540 & 0.1274 \\
\hline & \multicolumn{7}{c}{ Bin boundaries of $\pi^{0}$ momentum distribution $\left(|p|_{\pi^{0}}\right.$ in GeV $):$} \\
\cline { 2 - 8 }
\end{tabular}

note that the $\nu_{\mu}$ CCQE sample used in this work is different from the one used in the MiniBooNE first publication [190]. The values of the CCQE cross section parameters shown in Table 3.9 are therefore different to those detailed in [180], which correspond to the first oscillations result.

Uncertainties in single pion production and multiple pion production cross sections were assessed through the corresponding axial mass parameters $M_{A}^{1 \pi}$ and $M_{A}^{N \pi}$ in NUANCE. Uncertainties from the final state interactions model in NUANCE affecting primarily the level of $\pi^{+}$backgrounds in the $\nu_{\mu}$ CCQE sample were introduced through variations in the $\pi^{+}$charge exchange and absorption cross sections. The assumed uncertainty in the total $\pi^{+}$absorption cross section is $50 \%$ and for the charge exchange cross section 35\%. The strange quark contribution to the neutral current scattering is set with the parameter $\Delta s=0.0 \pm 0.1$. The deep inelastic scattering 
Table 3.9: NUANCE cross section parameters with uncertainties. The QE cross section parameters $M_{A}^{Q E}$ and $\kappa$ were determined as described in [191]. Parameters are assumed uncorrelated unless indicated in the table by the value of the correlation coefficient.

\begin{tabular}{ccc}
\hline \hline Parameter & Value & Correlations \\
\hline$M_{A}^{Q E}$ & $(1.2341 \pm 0.077) \mathrm{GeV}$ & $\rho\left(M_{A}^{Q E}, \kappa\right)=-0.875$ \\
$M_{A}^{1 \pi}$ & $(1.1 \pm 0.275) \mathrm{GeV}$ & $\rho\left(M_{A}^{1 \pi}, M_{A}^{\text {coh }}\right)=1$ \\
$M_{A}^{N \pi}$ & $(1.3 \pm 0.52) \mathrm{GeV}$ & NONE \\
$M_{A}^{\text {coh }}$ & $(1.030 \pm 0.275) \mathrm{GeV}$ & $\rho\left(M_{A}^{\text {coh }} M_{A}^{1 \pi}\right)=1$ \\
$\kappa$ & $(1.0220 \pm 0.0205)$ & $\rho\left(\kappa, M_{A}^{Q E}\right)=-0.875$ \\
$E_{B}$ & $(34 \pm 9) \mathrm{MeV}$ & $\mathrm{NONE}$ \\
$p_{F}$ & $(220 \pm 30) \mathrm{MeV}$ & NONE \\
$\Delta s$ & $(0.0 \pm 0.1) \mathrm{MeV}$ & NONE \\
Charge exchange & $50 \%$ & NONE \\
$\pi^{+}$absorption & $35 \%$ & NONE \\
\hline \hline
\end{tabular}

cross section is given a $25 \%$ uncertainty, and the radiative decay of the $\Delta$ resonance into photons is assigned a $9 \%$ uncertainty.

\subsection{Detector simulation}

The final state particles produced by the NUANCE event generator are passed to a GEANT3 [154] based Monte Carlo simulation that takes care of their transport across the oil medium and the production and propagation of individual photons, which are tracked from their production point until absorbed in the detector material. Photons that are absorbed in a PMT photocathode have the chance to produce a photoelectron. The PMT response and DAQ electronics are simulated separately. 
In the simulation the detector is a spherical iron shell with a small cylindrical neck at the top. The detector volume is filled with $\mathrm{CH}_{2}$ with density $\rho_{\text {oil }}=0.855 \mathrm{~g} / \mathrm{cm}^{3}$ used to model the oil. The tank is placed inside a vertical cylindrical concrete vault filled with air. The vault volume is contained in a larger cylindrical volume of dirt, modeled as a mixture of silicon (20\%), oxygen (65\%) and aluminum (15\%) atoms with total density $\rho_{\text {dirt }}=2.15 \mathrm{~g} / \mathrm{cm}^{3}$. A truncated cone of the same dirt material is placed above the dirt cylinder to model the overburden. A rendering of the detector and its surroundings is shown in Figure 3.13. The optical barrier separating the veto region from the inner tank is modeled as an aluminum sphere containing the four spherical flasks, the seven scintillator cubes, the PMTs and an array of pipes for PMT support arranged as circles of latitude parallel to the ground.

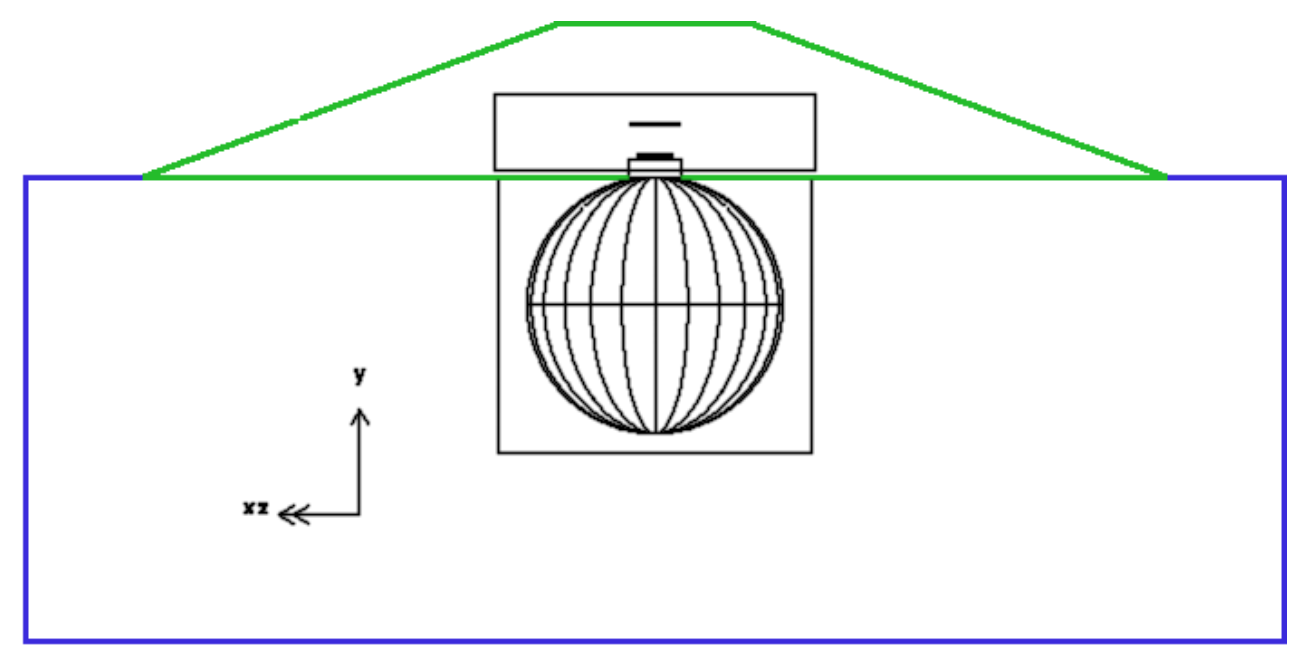

Figure 3.13: GEANT3 rendering of the detector, vault and surrounding dirt with overburden as defined in the detector Monte Carlo. The model is axially symmetric around the tank's vertical diameter.

The muon tracker system is also modeled and placed according to its surveyed position above the top hat. 


\section{Particle propagation and light production}

Details on the particle propagation and light production have been given elsewhere [180]. Suffice here to mention that GEANT3 routines have been used with the exception of the treatment of the decays $\pi^{0} \rightarrow \gamma e^{+} e^{-}$and $\mu \rightarrow e \nu \bar{\nu}$, for which routines using matrix elements have been developed ${ }^{3}$. Other non-standard modifications include proper implementation of the $\mu^{-}$capture rate on carbon [156] as well as modeling of hadronic interactions in the detector volume with the GCALOR [157] package.

The production of optical photons by charged particles moving through the oil is modeled with three mechanisms: (1) Cherenkov radiation, (2) intrinsic scintillation of the oil and (3) UV fluorescence, which we describe briefly below:

\section{Cherenkov radiation:}

Relativistic charged particles traversing a medium with index of refraction $n(\omega)$ with velocity $\beta \equiv v / c$, emit Cherenkov radiation at all frequencies $\omega$ satisfying the relation $\beta n(\omega)>1$. The coherent wavefront associated with radiation of each frequency is emitted at an angle $\cos \theta_{C}=1 / \beta n(\omega)$, and the number of photons emitted per unit path-length $x$, per unit wavelength $\lambda=2 \pi c / \omega$ is given by [11]:

$$
\frac{d^{2} N}{d x d \lambda}=\frac{2 \pi \alpha}{\lambda^{2}} \sin ^{2} \theta_{C}
$$

where $\alpha$ is the fine structure constant. H. O. Meyer from Indiana University measured the wavelength and temperature dependence of the index of refraction of the Marcol 7 oil used in MiniBooNE with an Abbe refractometer [155] and found it to be well described by:

\footnotetext{
${ }^{3}$ P. Meyers, Princeton University
} 


$$
\begin{array}{cc}
n(\lambda, T)=\left[n_{D}+B\left(\frac{1}{\lambda^{2}}-\frac{1}{\lambda_{D}^{2}}\right)\right]\left[1-\beta\left(T-T_{0}\right)\right] \\
n_{D} & 1.468 \pm 0.0002 \\
B & (4240 \pm 157) \mathrm{nm}^{2} \\
\beta & (3.66 \pm 0.04) \times 10^{-4}\left({ }^{\circ} \mathrm{C}\right)^{-1} \\
\lambda_{D} & 589.3 \mathrm{~nm} \\
T_{0} & 20.0^{\circ} \mathrm{C}
\end{array}
$$

Measured parameters for MiniBooNE Marcol 7 oil [155]

where $\lambda$ is the wavelength and $T$ is the temperature. The group velocity used to determine the velocity of photon propagation in the simulation is calculated from the phase velocity $v_{p}=c / n$ and dispersion relation $n(\omega)$, with $\omega \equiv 2 \pi c / \lambda$ derived from Eq.(3.20), as $v_{g}=v_{p} /\left(1+\frac{\omega}{n} \frac{d n}{d \omega}\right)$.

\section{Scintillation and UV fluorescence:}

Energy deposition of charged particles traversing the oil produces the excitation of organic molecules, whose subsequent de-excitation over timescales of 10's of ns is a source of isotropic and delayed light emission known as scintillation light (see Figure 2.7 in Section 2.4). The excitation of organic molecules can be also produced by UV photons (as opposed to particle tracks), in which case the term fluorescence is used. UV fluorescence is a wavelength-shifting process by which an otherwise undetectable UV photon ${ }^{4}$ can produce optical photons that the PMTs can detect. The number of scintillation or fluorescence photons per energy deposited in the oil medium is simulated according to the expression [11]

$$
\frac{d N_{\mathrm{sci}}}{d E}=\frac{31.64 \mathrm{MeV}^{-1}}{1+B_{1}\left(\frac{1}{\rho_{\mathrm{oi} 1} \frac{d E}{d x}}\right)+B_{2}\left(\frac{1}{\rho_{\mathrm{oi} 1} \frac{d E}{d x}}\right)^{2}}
$$

with the coefficients set to the values

\footnotetext{
${ }^{4}$ The PMTs are not sensitive to photons with $\lambda \lesssim 280 \mathrm{~nm}$
} 


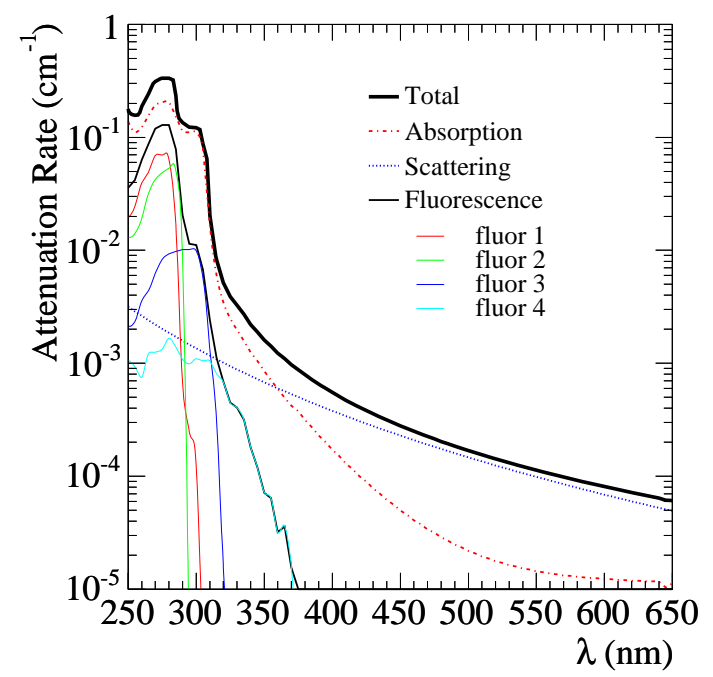

Figure 3.14: The photon attenuation rates in mineral oil as a function of photon wavelength $\lambda$. The contributions from absorption, scattering, and fluorescence are also shown. Also shown are the the contributions to the fluorescence rate from each of the four fluors included in the simulation.

$$
\begin{aligned}
& B 1=0.014 \mathrm{~g} \mathrm{MeV}^{-1} \mathrm{~cm}^{-2} \\
& B 2=0.000 \mathrm{~g} \mathrm{MeV}^{-1} \mathrm{~cm}^{-4}
\end{aligned}
$$

The coefficient $B_{2}$ is included in the simulation to assess systematic uncertainties. This expression (Birk's Law) estimates the light output from a single fluorophore correcting for the ionization loss of tracks in the medium. The scintillation yield constant 31.64 photons per MeV in Eq.(3.21) was determined experimentally to have an exponentially decaying emission with time constant of $\tau=34$ ns [158]. A total of four fluorophores (fluors for short) were detected in studies of time resolved fluorescence [159] of the Marcol 7 oil by D. Toptygin from John Hopkins University. The optimal settings for the photon yields from each fluor were determined by comparing data and simulation distributions from samples of events rich in scintillation light (NC elastic scattering sample [184] and Michel electron samples). It was found that the data preferred a model in which scintillation light comes only from a single fluor (fluor 4 in 
Figure 3.14). The remaining three fluors were simulated and used in the systematic error assessment.

Photon absorption, scattering, and fluorescence affect the propagation of photons traveling across the tank. The rates of these processes are shown in Figure 3.14

\section{External interactions}

Neutrino interactions with the material surrounding the detector were simulated by extending the radius of event generation to a large volume 15 meters in radius, concentric with the detector and within the dirt volume described in Section 3.3. These events contribute significantly to the misidentification background of the $\nu_{\mu} \rightarrow \nu_{e}$ oscillations search, and typically produce a $\pi^{0}$ via a $\mathrm{NC}$ interaction which promptly decays into two gammas, one of them finding its way to the interior of the tank, as schematically represented in Figure 3.15(a).

Events from external interactions tend to deposit all of their energy near the tank wall, and their reconstructed tracks tend to point toward the interior of the detector ${ }^{5}$. A dedicated sample enhanced in dirt events was used to measure the observed rate of these interactions. Details on the measurement can be found elsewhere [160]. Figure 3.15 shows the measurement as a distribution of visible energy deposited by the events in the dedicated dirt sample. The dirt contribution is shown in red and is consistent with the Monte Carlo prediction within the measurement uncertainty.

\section{Beam-off background simulation}

Activity from random triggers coming primarily from cosmic ray muons and their decay electrons, and dark noise is recorded at 2.01 Hz. PMT hits from this triggers

\footnotetext{
${ }^{5}$ The direction vector $U$ and radial vertex position $R$ of dirt events tend to point in opposite directions: $U \cdot R<0$.
} 


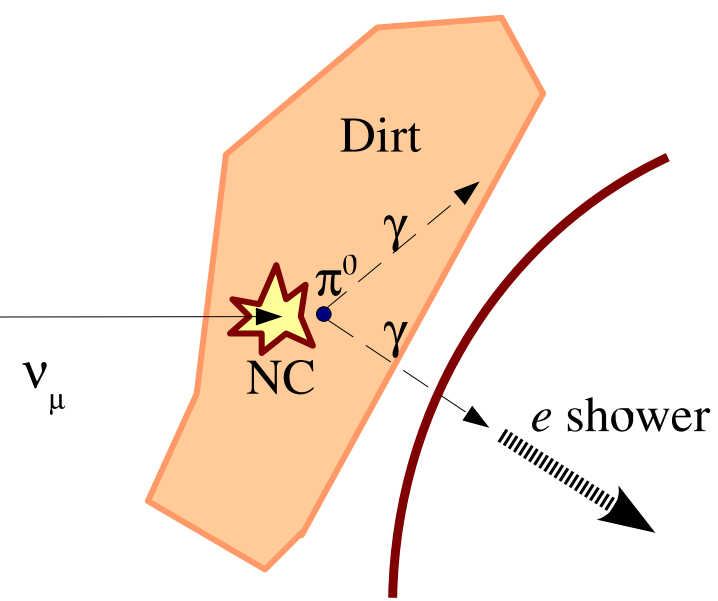

(a) Dirt event illustration.

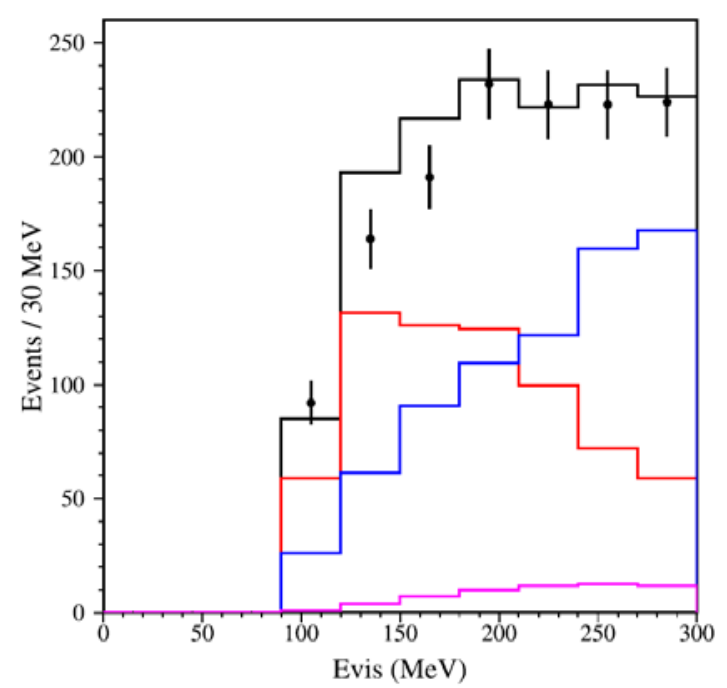

(c) Visible energy distribution.

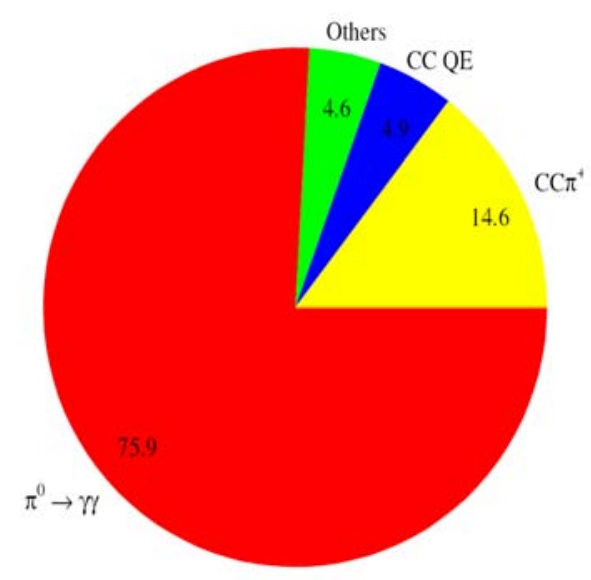

(b) Sample composition.

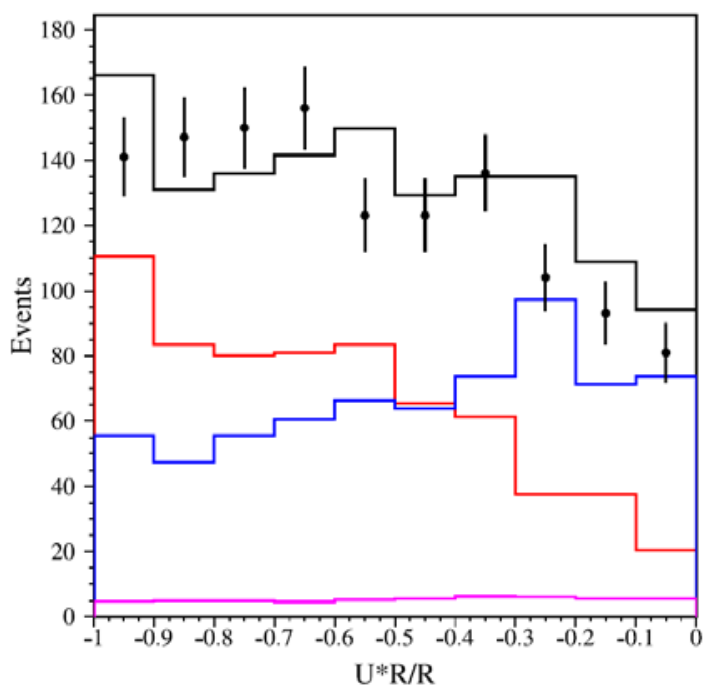

(d) $U \cdot R / R$ distribution.

Figure 3.15: Events from external interactions. In (a) an illustration of an external interaction. In (b) the composition if the sample in terms of neutrino interaction type. In (c) and (d) the visible energy and $U \cdot R / R$ distribution of a sample of events enhanced in dirt events, respectively. The points with error bars show the data with statistical uncertainties, the solid red line shows the contribution from the dirt events, and the solid blue line shows the contribution from events occurring within the tank walls. The black line is the sum of the dirt plus backgrounds. Taken from [160]. 
Table 3.10: Number of parameters associated with the various optical properties of the detector simulation. The 35 parameters are varied to assess systematic uncertainties from the modeling of optical properties. Taken from [180].

\begin{tabular}{lc|lc}
\hline \hline Property & $\#$ par. & Property & \# par. \\
\hline extinction length & 5 & Scattering (Rayleigh/Raman) & 3 \\
index of refraction & 3 & PMT angular efficiency & 2 \\
fluor scintillation yield & 4 & fluor fluorescence yield & 4 \\
fluor UV fluorescence yield & 4 & fluor time constant & 4 \\
Cherenkov scale factor & 1 & reflections & 2 \\
Birks' law coefficients & 2 & old/new PMT relative efficiency & 1 \\
\hline \hline
\end{tabular}

are mixed with PMT hits from the Monte Carlo simulation to account for beam unrelated activity in a process called "strobe merge". Hits from the random trigger that coincide in channel number with hits from simulated events are combined on a single pulse in that channel.

\subsubsection{Detector simulation uncertainties}

A 35-dimensional parameter space is used to represent all possible variations in the optical properties of the detector. Table 3.10 shows the number of parameters controlling each of the various optical properties in the simulation. The covariance matrix relating all the parameters in Table 3.10 is formed with the procedure originally described in [180] which is summarized here for completeness. A starting estimate of the covariance matrix $\mathbf{S}$ and mean $\hat{\mathbf{s}}=\left(\hat{s}_{1}, \ldots, \hat{s}_{35}\right)$ of all the optical model parameters is constructed. Assuming a multivariate Gaussian distribution for the parameters we draw $M \sim 3000$ random Monte Carlo parameter sets $\mathbf{s}^{(\mathbf{m})}, m=1, \ldots, M$, generating for each one of them a simulated sample of Michel electrons in the detector. We 
compare the agreement between data and each of the $M$ simulations by calculating the $\chi^{2}$ of a given reconstructed distribution. For an ideal simulation, $\chi^{2}=N_{\text {dof }}$ is the number of bins used in the comparison. The likelihood that the difference between the data and the given simulation is due only to statistical fluctuations is calculated as

$$
p=\exp \left(-\frac{1}{2}\left(\chi^{2}-N_{\mathrm{dof}}\right)\right)
$$

and compare it to the likelihood to draw that parameter set according to the matrix $\mathrm{S}:$

$$
w=\exp \left(-\frac{1}{2}\left(\mathbf{s}^{(m)}-\hat{\mathbf{s}}\right)^{\mathrm{T}} \mathbf{S}^{-1}\left(\mathbf{s}^{(m)}-\hat{\mathbf{s}}\right)\right) .
$$

The constraint from the data is introduced by defining a weight for each drawn parameter set

$$
\eta \equiv \min \left(1, \frac{p}{w}\right)
$$

with which we construct a new multivariate Gaussian distribution with mean

$$
\hat{\mathbf{s}}^{\prime}=\frac{\sum_{m} \eta_{m} \mathbf{s}^{(m)}}{\sum_{m} \eta_{m}}
$$

and covariance matrix

$$
S_{i j}^{\prime}=\frac{\sum_{m} \eta_{m}\left(s_{i}^{(m)}-\hat{s}_{i}^{\prime}\right)\left(s_{j}^{(m)}-\hat{s}_{j}^{\prime}\right)}{\frac{M-1}{M} \sum_{m} \eta_{m}}
$$

Using this new Gaussian distribution the process is repeated using new test distributions (energy, timing, position, etc.) in the $\chi^{2}$ calculation until data/simulation agreement ceases to improve. About 20 iterations were computed requiring $\sum_{m} \eta_{m}>$ 300 at each iteration step. 


\subsection{Event Reconstruction algorithms}

The experiment uses two main reconstruction algorithms, the S-Fitter and the PFitter, which differ primarily in the detail with which they model light emission by extended tracks like those of muons, and the amount of time and effort devoted to fitting an event under the $\pi^{0}$ hypothesis. The P-Fitter can be seen as an improved version of the S-Fitter.

In both reconstruction algorithms an event is characterized by a set of parameters $\vec{\alpha}$ (position, direction, energy, etc.). The likelihood for measuring a set of charges $q_{i}$ and times $t_{i}$ in a given event is calculated as the product of the individual time and charge likelihoods of all the PMTs:

$$
L_{\text {event }}=\prod_{i=1}^{1280} L_{q}\left(q_{i} ; \vec{\alpha}\right) L_{t}\left(t_{i} ; \vec{\alpha}\right)
$$

where $L_{q}$ and $L_{t}$ represent the probability of measuring a charge $q$ and a time $t$ at any $\mathrm{PMT}^{6}$. $L_{\text {event }}$ is the likelihood that the event is described by the set of parameters $\vec{\alpha}$ given the set of charges and times $\left(q_{i}, t_{i}\right)$. The optimal values of the parameters are found by maximizing the likelihood $L_{\text {event }}$ with respect to the parameters $\vec{\alpha}$.

\subsubsection{S-Fitter: electron reconstruction}

The S-Fitter uses a point-like source model to describe electron tracks. An electron track is fully characterized by a four-vertex $(x, y, z, t)$ in the detector coordinate system, a direction $(\theta, \phi)$, and a kinetic energy $(E)$, this is $\vec{\alpha}=(x, y, z, t, \theta, \phi, E)$.

\footnotetext{
${ }^{6}$ The description given here is based on the S-Fitter, from which the P-Fitter was constructed. Both share many of the same principles and differ on the details. For the precise definition of the event likelihood in the P-Fitter reconstruction see [180].
} 
Cherenkov light from a relativistic electron is modeled to have a strength $\rho$ (photons per steradian), modulated by an energy dependent angular distribution function $F\left(\cos \theta_{e}, E\right)$ where $\theta_{e}$ is the photon emission angle with respect to the electron track direction. The angular distribution is normalized such that

$$
\int_{-1}^{1} F\left(\cos \theta_{e}, E\right) d \cos \theta_{e}=1 .
$$

As a consequence of the point-like source approximation, the angular distribution $F\left(\cos \theta_{e}, E\right)$ broadens with increasing electron energy for longer tracks. Natural isotropic scintillation light from the oil and scattered Cherenkov light are emitted with a strength $\Phi$ (photons per steradian). Both $\rho$ and $\Phi$ are proportional to the event energy $E$, and the proportionality constants are determined from fits to the charge distributions of Michel electron data [114].

\section{Raw event position and time}

A typical track in the tank produces photons that hit a certain number $N_{\text {hit }}$ of PMTs. The charge and time $\left(q_{i}, t_{i}\right), i=1, \ldots, N_{\text {hit }}$ of each PMT hit is the basic information for the event reconstruction. A first guess of the position of the event is calculated from the charge-averaged position of the PMTs that are hit:

$$
\mathbf{r}=\frac{1}{Q} \sum_{i=1}^{N_{h i t}} q_{i} \mathbf{R}_{i}, \quad Q=\sum_{i=1}^{N_{\mathrm{hit}}} q_{i}
$$

With this first guess position, a first guess of the time of the event is calculated as the charge-averaged time of each hit, corrected for the photon time of flight.

$$
t=\frac{1}{Q} \sum_{i=1}^{N_{\mathrm{hit}}} q_{i}\left(t_{i}-\left|\mathbf{R}_{i}-\mathbf{r}\right| / c_{n}\right)
$$

where $c_{n}$ is the speed of light in the oil with index of refraction $n$. This first guess of the time and position of the event are used to seed a detailed reconstruction maximum likelihood algorithm described next. 


\section{Time Likelihood and the fast fit}

For each track hypothesis the simulation is used to produce the distribution of corrected times $T_{c}\left(t_{\text {corr }}^{g}\right)$, where $t_{\text {corr }}^{g}=t^{g}-t_{i}+\left|\mathbf{R}_{i}-\mathbf{r}^{g}\right| / c_{n}$ is the true generated corrected time distribution, depending on the true generated event time $t^{g}$ and position $\mathbf{r}^{g}$, and the time of each hit $t_{i}$. The distribution $T_{c}$ has a Gaussian component from the prompt Cherenkov light and an exponential component folded with a Gaussian of equal width corresponding to the scintillation light. The common width of the Gaussian used in this step is $\sigma_{\text {eff }} \sim 2 \mathrm{~ns}$, and the exponential component has a time constant of $\tau_{\text {eff }} \sim 20 \mathrm{~ns}$, having relative amplitudes of 0.57 (Cherenkov) and 0.43 (scintillation). This simple model of the corrected time distribution $T_{c}\left(t_{c o r r}\right)$, is used to form a primitive time likelihood for events with unknown time and position, whose corrected time is $\left.t_{\text {corr }}=t-t_{i}+\left|\mathbf{R}_{i}-\mathbf{r}\right| / c_{n}\right)$

$$
\mathcal{L}=\prod_{i=1}^{N_{\text {hit }}} T_{c}\left(t_{\text {corr }, i}\right)
$$

A better estimate of the event time and position is obtained by maximizing this time likelihood function with respect to the position $\mathbf{r}$ and the time $t$, using as starting values the values guessed in Eqs.(3.29) and (3.30). This better estimate of the position is then used to determine the track direction as the charge-averaged direction of the emitted photons:

$$
\begin{aligned}
\mathbf{U} & =\frac{1}{Q} \sum_{i=1, \text { prompt }}^{N_{\text {hit }}} q_{i}\left(\mathbf{R}_{i}-\mathbf{r}\right) \\
\hat{\mathbf{u}} & =\mathbf{U} /|\mathbf{U}| \text { normalized direction }
\end{aligned}
$$

where the sum is over the prompt hits defined as those with $t_{c o r r}<2.5 \sigma_{\text {eff }}$. At this stage, a first estimate of the event energy is calculated from the total charge with a radial position dependent correction determined from control data sample of Michel 
electrons:

$$
E(Q, r)=\frac{Q}{F(r)}, \quad F(r)=\left(3.17+0.0283 \exp \left\{\frac{r}{1.344 \mathrm{~m}}\right\}\right)\left(\frac{\mathrm{PE}}{\mathrm{MeV}}\right)
$$

This radial correction empirically takes into account the attenuation length and solid angle effects in the detector and is obtained from fits to the charge distribution of Michel electron data in radial shells and provides the charge-to-energy conversion. This reconstructed event energy determines the Cherenkov angular distribution and the corrected time distribution, as well as the strengths of Cherenkov and scintillation light emission. All of these quantities are used in the next minimization step.

\section{The full fit}

The full fitting algorithm starts with a prediction $\mu^{i}$ of the number of PE in each hit PMT, which will have a contribution from prompt Cherenkov light $\mu_{c}^{i}$ as well as delayed scintillation light $\mu_{s}^{i}$. Both contributions to the total charge $\left(\mu^{i}=\mu_{c}^{i}+\mu_{s}^{i}\right)$ in a PMT depend on the solid angle $\Omega_{i}$ subtended by the PMT from the event vertex, the PMT relative quantum efficiency ${ }^{7} \epsilon_{i}$, and the corresponding attenuation length $\lambda$ for light in the oil $\left(\lambda_{c e r}=24.4 \mathrm{~m}, \lambda_{s c i}=16.4 \mathrm{~m}\right)$. In addition, the predicted amount of charge from Cherenkov photons depends on the angular distribution of photons emitted for a given track $F\left(\cos \theta_{e}, E\right)$ described above. We predict the number of PE from Cherenkov and scintillation light reaching a PMT as:

$$
\begin{aligned}
\mu_{c}^{i} & =\rho \Omega_{i} \exp \left(-\left|\mathbf{R}_{r}-\mathbf{r}\right| / \lambda_{c e r}\right) \times \epsilon_{i} F(\cos \theta, E) \\
\mu_{s}^{i} & =\Phi \Omega_{i} \exp \left(-\left|\mathbf{R}_{r}-\mathbf{r}\right| / \lambda_{s c i}\right) \times \epsilon_{i}
\end{aligned}
$$

where absorbing the factor $\pi\left|\mathbf{R}_{r}-\mathbf{r}\right|^{2}$ in the definition of the fluxes $\rho$ and $\Phi$, the solid angle $\Omega_{i}$ is given in terms of the PMT angular acceptance $f(\cos \eta)^{8}$, and the

\footnotetext{
7 The absolute values can be absorbed in the definition of the fluxes $\rho$ and $\Phi$.

${ }^{8} \eta$ is the incoming photon angle with respect to the normal to the PMT face.
} 
event radial distance from the PMT:

$$
\Omega_{i}=f(\cos \eta) /\left|\mathbf{R}_{i}-\mathbf{r}\right|^{2}
$$

Using the simulation, corrected time distributions for various values of the predicted charge $\mu$ are calculated and separated into a Cherenkov and scintillation components $T_{c}\left(t_{c o r r}, \mu_{i}\right)$ and $T_{s}\left(t_{c o r r}, \mu^{i}\right)$. A detailed time likelihood function is defined for each hit with predicted charge $\mu^{i}$ and corrected time $t_{\text {corr }}^{i}$ as:

$$
\begin{aligned}
\mathcal{L}_{T}^{i}\left(t_{\text {corr }}^{i}, \mu^{i}\right) & =x^{i} T_{c}\left(t_{\text {corr }}^{i}, \mu^{i}\right)+(1-x) T_{s}\left(t_{\text {corr }}^{i}, \mu^{i}\right), \\
t_{\text {corr }}^{i} & \left.=t-t_{i}+\left|\mathbf{R}_{i}-\mathbf{r}\right| / c\right)
\end{aligned}
$$

where $x^{i}=\mu_{c}^{i} /\left(\mu_{c}^{i}+\mu_{s}^{i}\right)$ is the predicted fraction of Cherenkov charge in PMT $i$, and $t_{\text {corr }}$ is the corrected time of the hit. A charge likelihood $\mathcal{L}_{q}^{i}$, defined as the probability to measure a charge $q_{i}$ when expecting a charge $\mu_{i}$ in PMT $i$, is extracted from a set of laser calibration events [114] by recording a two dimensional histogram of all the possible values of the measured and predicted charge $(q$ and $\mu)$ and enforcing the normalization condition $\int \mathcal{P}(q, \mu) d q=1$.

The total likelihood of a hit is simply the product of the time likelihood $\mathcal{L}_{T}^{i}$ and the charge likelihood $\mathcal{L}_{q}^{i}$, and the total Likelihood to be maximized for the event is

$$
\mathcal{L}_{Q, T}(\mathbf{r}, t)=\prod_{i=1}^{N_{\text {hit }}} \mathcal{L}_{T}^{i}\left(t_{\text {corr }}^{i}, \mu^{i}\right) \times \mathcal{L}_{q}^{i}\left(q_{i}, \mu^{i}\right)
$$

Maximization of this likelihood function with respect to the event time and position gives a much better reconstruction of the event. The vertex that is calculated from this procedure corresponds to the mean photon emission point $(\mathrm{M} \gamma \mathrm{EP})$ assumed in the model, which has a systematic shift that is corrected for to obtain the event vertex. The reconstructed event vertex is calculated by shifting the reconstructed $\mathrm{M} \gamma \mathrm{EP}$ backward along the track direction by an amount dependent on the energy 
of the track ${ }^{9}$. This energy dependent correction is also extracted from the Michel electron control sample. The electron vertex reconstruction resolution is $\sim 30 \mathrm{~cm}$.

\section{Flux Fit}

A final minimization step is performed keeping the 4-vertex and direction found in the full fit fixed, but varying the Cherenkov and scintillation light strengths $\rho$ and $\Phi$. The charge to energy conversion factors change throughout the detector volume as a consequence of PMT angular coverage as well as light attenuation and scattering. A radial correction as that in Eq.(3.32) are applied giving an energy resolution at the Michel energy spectrum end-point (52.3) $\mathrm{MeV}$ of $\sim 13 \%$.

\subsubsection{S-Fitter: TRK (muon) reconstruction}

Even when muons produce tracks that are typically much longer than electron tracks the four-vertex and energy calculated with the single $\mathrm{M} \gamma \mathrm{EP}$ model used for electrons gives a reasonably good position ( $\sim 28 \mathrm{~cm}$ uncertainty), direction $\left(\sim 10^{\circ}\right.$ uncertainty), and energy ( $\sim 5 \%$ uncertainty at $1 \mathrm{GeV}$ muon energy) resolution. In order to obtain a good electron/muon discriminant the following simple approach is taken: once the event has been reconstructed using the point-like model, the reconstructed $\mathrm{M} \gamma \mathrm{EP}$ and reconstructed direction are kept fixed, while the central light source is replaced by two identical sources placed symmetrically around the middle point on the track. The minimization algorithm determines the optimal distance between the sources and their strengths. The fitted separation, labeled $\mathrm{TRK}^{10}$, is used as a discriminant to separate electron tacks from muon tracks.

\footnotetext{
${ }^{9} s=203.8-156.7 \exp (-E / 995.96 \mathrm{MeV})$, expressed in $\mathrm{cm}$

10 This quantity is not properly a track length, but is proportional to the spatial extension of the event.
} 


\subsubsection{S-Fitter: $\pi^{0}$ reconstruction}

A $\pi^{0}$ decays promptly into two photons which upon traveling a certain distance in the oil medium photo-convert producing each a shower of electrons and positrons. Each shower produces a light pattern similar to that of a single electron with a Cherenkov ring plus some scintillation light. The model used to fit these events consists of two electron models with displaced vertices. The fit has 14 parameters in the set $\vec{\alpha}$ : the $\pi^{0}$ four-vertex $(x, y, z, t)$, two directions $\left(\phi_{1}, \theta_{1}\right)$ and $\left(\phi_{2}, \theta_{2}\right)$ for the two photons, two shifts along these directions $s_{1}$ and $s_{2}$, representing the conversion distances from the $\pi^{0}$ vertex, and the scintillation and Cherenkov strengths for the two rings $\left(\rho_{1}, \Phi_{1}\right)$, and $\left(\rho_{2}, \Phi_{2}\right)$. These parameters are adjusted in three minimization steps which consist on combination of fixed and free parameters which follow an initialization phase to get the optimal starting values of all variables.

The event four-vertex and position from the electron algorithm described above are used as the initial set of parameters for the most energetic of the two photons (labeled 1 here unto). This sets the initial values of $(x, y, z, t),\left(\phi_{1}, \theta_{1}\right)$ and $\rho_{1}$. The energy and direction of the second photon are initialized by performing a grid search over $\sim \mathcal{O}(100)$ directions distributed uniformly over the $4 \pi$ solid angle around the first photon track and finding the direction and energy $\left(E_{2} \propto \rho_{2}\right)$ that maximizes the amount of charge contained in the second Cherenkov cone giving the initial values or $\left(\phi_{2}, \theta_{2}\right)$ and $\rho_{2}$. The scintillation fractions are ignored in the first two steps of the three-step minimization process described below:

1. An unconstrained fit returns the $\pi^{0}$ four-vertex $(x, y, z, t)$, the shifts to the two photons $(s 1, s 2)$, the directions of the two photons $\left(\phi_{1}, \theta_{1}\right)$ and $\left(\phi_{2}, \theta_{2}\right)$, and the fraction of Cherenkov light carried by the most energetic photon $f_{\text {cer }}=$ $\rho_{1} /\left(\rho_{1}+\rho_{2}\right)$. The two scintillation strengths are ignored. 
2. The four-vertex of the $\pi^{0}$ is held fixed, the scintillation strengths are ignored, and a second iteration of the directions $\left(\phi_{1}, \theta_{1}\right),\left(\phi_{2}, \theta_{2}\right)$, the shifts $s_{1}$ and $s_{2}$, and the Cherenkov strengths $\rho_{1}$ and $\rho_{2}$ are obtained.

3. The four-vertex position, the direction of the two photons, and the conversion shifts of the two photons $s_{1}$ and $s_{2}$ are kept fixed with the values obtained in the previous step, while varying the Cherenkov and scintillation strengths $\left(\rho_{1}, \Phi_{1}\right)$ and $\left(\rho_{2}, \Phi_{2}\right)$.

This procedure yields a spatial resolution of the $\pi^{0}$ vertex of $\sim 50 \mathrm{~cm}$. The reconstructed energy of the photons $E_{1}$ and $E_{2}$ are determined directly from the fitted Cherenkov light strengths of the two cones $\rho_{1}$ and $\rho_{2}$ after the third step, and are used together with the angle between the two reconstructed directions $\alpha_{\gamma \gamma}$ to give the reconstructed $\pi^{0}$ mass:

$$
M_{\pi^{0}}^{2}=2 E_{1} E_{2}\left(1-\cos \alpha_{\gamma \gamma}\right)
$$

The $\pi^{0}$ mass reconstruction has a resolution of $\sim 25 \mathrm{MeV}$. For a more detailed description of this algorithm see [113] and [183].

\subsubsection{P-Fitter: electron/muon single track reconstruction}

The P-Fitter ${ }^{11}$ has been extensively described elsewhere [180]. The algorithm uses a more detailed light emission model by introducing photon emission profiles for Cherenkov $\left(\rho_{\mathrm{Ch}}(s)\right)$ and scintillation $\left(\rho_{\text {sci }}(s)\right)$ light, which depend on the distance $s$ along the track from its vertex, and on the event energy $E_{0}$. The calculation of the predicted charge in a PMT involves an integral of the emission profile along the track

\footnotetext{
${ }^{11}$ Developer Ryan B. Patterson, Princeton University
} 
convoluted with a PMT acceptance function $J(s)$ :

$$
\begin{aligned}
\mu_{\mathrm{Ch}} & \propto \int_{-\infty}^{\infty} \rho_{\mathrm{Ch}}(s) J(s) F(\cos \theta(s) ; s) d s, \\
\mu_{\mathrm{sci}} & \propto \int_{-\infty}^{\infty} \rho_{\text {sci }}(s) J(s) d s .
\end{aligned}
$$

The function $J(s)$ is approximated by a parabola $J(s) \approx j_{1}+j_{2} s+j_{3} s^{2}$ and parameterized with the coefficients $\left\{j_{i}\right\}$ determined from evaluating $J(s)$ at three points along the track: the vertex $s=0$, the mid point $s=\Delta s_{\text {mid }}$, and the end point $s=2 \Delta s_{\text {mid }}$. For the Cherenkov light contribution, the angular distribution of photons $F\left(\cos \theta(s) ; s, E_{0}\right)$ depends on the distance along the track $s^{12}$ as well as the event energy $E_{0}$. Tables of the integrals

$$
\begin{aligned}
I_{i}^{\mathrm{sci}}\left(E_{0}\right) & =\int_{-\infty}^{\infty} d s s^{n} \rho_{\mathrm{sci}}\left(s, E_{0}\right) \\
\mathcal{I}_{i}^{\mathrm{Ch}}\left(E_{0}\right) & =\int_{-\infty}^{\infty} d s s^{n} \rho_{\mathrm{Ch}}\left(s, E_{0}\right) F\left(\cos \theta(s) ; s, E_{0}\right), \quad \text { for } i=0,1,2,
\end{aligned}
$$

for a wide range of energies are calculated beforehand and made available to the code.

Similar tables are constructed for the indirect contribution to the Cherenkov and scintillation light yields coming from processes like reflections, scattering and fluorescence.

The P-Fitter constructs a time likelihood function for each hit using a similar convention as the S-Fitter in Eq.(3.35), in that it uses the mean of the emission profile (the track "mid-point") in the definition of the corrected time:

$$
t^{c}=t-T_{0}-\frac{r\left(\Delta s_{\text {mid }}\left(E_{0}\right)\right)}{c_{n}}-\frac{\Delta s_{\text {mid }}\left(E_{0}\right)}{c},
$$

where $t$ is the time measured hit, $T_{0}$ is the track starting time, $\Delta s_{\text {mid }}\left(E_{0}\right)$ is the mean of the Cherenkov emission profile for a track of energy $E_{0}, r\left(\Delta s_{\operatorname{mid}\left(\mathrm{E}_{0}\right)}\right)$ is the

\footnotetext{
${ }^{12}$ The profile of Cherenkov light emission changes as the particle advances and loses energy, and the angle $\theta(s)$ depends on which part of the track we are in.
} 
distance to the PMT from the track mid point, $c$ is the speed of light, assumed to be the particle velocity, and $c_{n}$ is, as before, the speed of light in the oil. As mentioned before, the time likelihood p.d.f. used in the P-Fitter is similar to that of the S-Fitters but it constructs the prompt (Cherenkov) and late (scintillation) contributions using a different procedure detailed in [180]. The charge likelihood is calculated assuming Poisson statistics for each hit.

In addition, all the required quantities, the look-up tables of the integrals $\mathcal{I}_{i}^{\mathrm{Ch}}$ and $I_{i}^{\text {sci }}$, as well as the time likelihood distribution components are calculated for the two hypotheses of muon and electron tracks.

This detailed model of photon emission from extended tracks gives the P-Fitter significant power to reconstruct muon tracks over the S-Fitter, while the electron tracks are reconstructed with comparable resolution with both algorithms.

\subsubsection{P-Fitter: $\pi^{0}$ reconstruction}

A two-track extension of the single-track algorithm of the P-Fitter is used to fit the 12 parameters defining a $\pi^{0} \rightarrow \gamma \gamma$ event: the four-vertex of the $\pi^{0}(x, y, z, t)$, the two conversion distances $s_{1}$ and $s_{2}$ of the photons, and the energy and direction of each photon $E_{i},\left(\phi_{i}, \theta_{i}\right), i=1.2$. As opposed to the S-Fitter, no fit for the Cherenkov and scintillation strengths is made, since these are obtained from pre-calculated look-up tables.

The main difference between the P-Fitter and S-Fitter $\pi^{0}$ reconstruction algorithms is the amount of time spent sampling the parameter space and the care taken to avoid trapping scenarios where the minimization of the negative log of the likelihood function falls into local minima and stops sampling possibly better regions of the parameter space. The procedure devised by Patterson [180] uses Monte Carlo simu- 
lated $\pi^{0}$ events to study all possible scenarios that can lead to the fit getting trapped in pathological configurations and finds a collection of initializations of the seeds to start the minimization that resolves most of the pathologies. These are summarized below:

- Four possible pairs of conversion lengths $s_{1}$ and $s_{2}$ are tried by seeding each to either $50 \mathrm{~cm}$ or $250 \mathrm{~cm}$.

- Nine starting directions for the first photon $\left(\phi_{1}, \theta_{1}\right)$ are tried starting with the result of a one-track fit under the electron hypothesis (e-fit), followed by eight perturbations extracted from the covariance ellipse of the fitted direction.

- The direction of the second photon $\left(\phi_{2}, \theta_{2}\right)$ is searched over a grid of $24 \phi$ steps and $12 \theta$ steps which can be made finer (50 and 25) in case of failure to find the second track.

- The four-vertex of the $\pi^{0}$ is seeded with the $e$-fit vertex, shifted according to $s_{1}, \phi_{1}$, and $\theta_{1}$.

- The energy of the most energetic photon $E_{1}$ is seeded with approximately the $e$-fit energy, and that of the second photon $E_{2}$ is set to that needed to give the mass of the $\pi^{0}$ as in Eq.(3.36)

The $\pi^{0}$ fit can be run with the additional constraint of yielding the invariant mass $M_{\pi^{0}}$. This is implemented by removing the energy of the second photon as a free parameter and fixing it to $E_{2}=M_{\pi^{0}}^{2} /\left[E_{1}() 1-\cos \alpha_{\gamma \gamma}\right]$. The fixed-mass fit the standard $\pi^{0}$ hypothesis whose likelihood value $\mathcal{L}_{\pi^{0}}$ is used as a particle identification variable, while the free mass fit allows for the reconstructed $M_{\pi^{0}}$ value to be used as a second PID variable. 
The result of all these initializations is a significantly slower $\pi^{0}$ fitter $(\sim \times 10$ slower than S-Fitter), but with a much higher power to distinguish $\pi^{0}$ events from electron-like events. 


\subsection{Particle Identification Methods}

The detector is able to identify particles by means of the topology of their light emission patterns projected onto the tank walls and the time distribution of the activity produced by their tracks. Only charged particles able to ionize the medium producing either Cherenkov and/or scintillation light are visible to the detector. Typically, muons have a sharp outer Cherenkov ring that in as it advances along its track, electrons have a diffuse Cherenkov ring effected by multiple Coulomb scattering of the electron track, the ring is typically not filled in because electron tracks are very short (a few tens of centimeters long). A third class of events that are relevant are $\mathrm{NC}$ $\pi^{0}$ events which decay promptly $\left(\tau \sim 8.4 \times 10^{-17} \mathrm{~s}\right)$ into two photons, which photoconvert as they travel through the oil producing two electron-like rings in a single event. These three types of event topologies are illustrated in Figure 3.16.
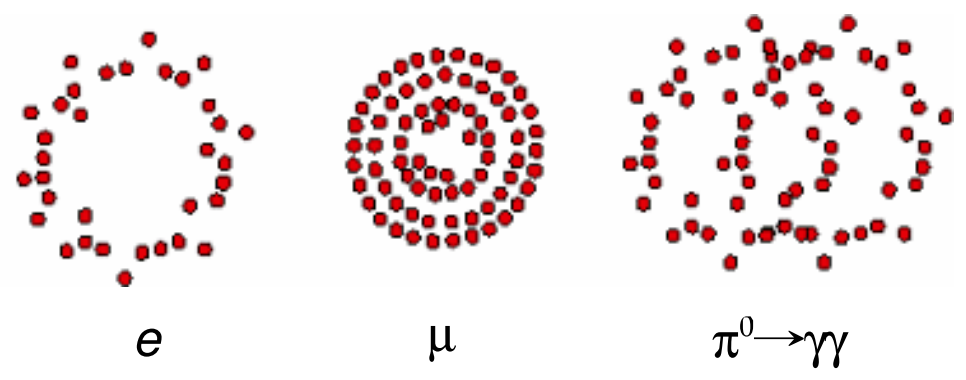

Figure 3.16: Schematic of typical hit topologies of electrons, muons, and $\mathrm{NC} \pi^{0}$ events in the MiniBooNE detector.

\subsubsection{Track Based Likelihood (TBL) particle identification}

The P-Fitter described in sections 3.4.4 to 3.4.5 provides a powerful way to distinguish electrons from muons and electrons from $\pi^{0}$ events. Best-fit track parameters and the corresponding maximum likelihoods are extracted under three different hypotheses: 


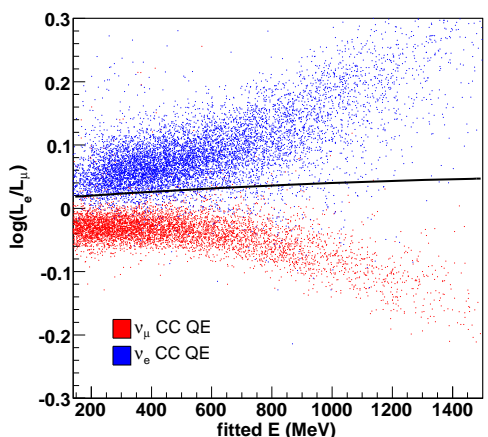

(a) $e / \mu$ cut

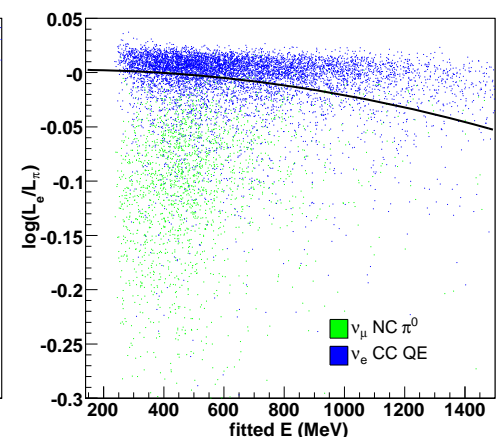

(b) $e / \pi^{0}$ cut

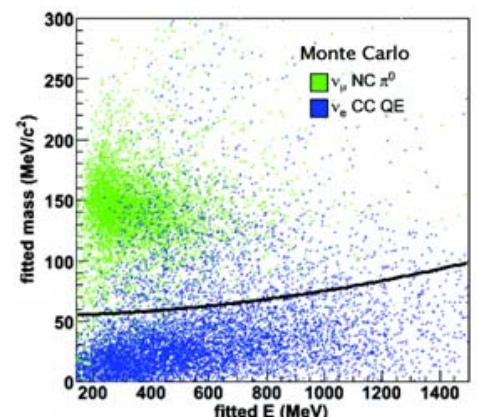

(c) $\pi^{0}$ mass cut $\left(M_{\gamma} \gamma\right)$

\begin{tabular}{lll}
\hline \hline $\log \left(\mathcal{L}_{e} / \mathcal{L}_{\mu}\right)$ & $\log \left(\mathcal{L}_{e} / \mathcal{L}_{\pi^{0}}\right)$ & $M_{\gamma \gamma}$ \\
$>a_{0}+a_{1} E_{e}+a_{2} E_{e}^{2}$ & $>b_{0}+b_{1} E_{e}+b_{2} E_{e}^{2}$ & $<c_{0}+c_{1} E_{e}+c_{2} E_{e}^{2}$ \\
\hline$a_{0}: 1.355 \times 10^{-2}$ & $b_{0}: 2.471 \times 10^{-3}$ & $c_{0}: 3.202 \times 10^{-2} \mathrm{GeV}^{-2}$ \\
$a_{1}: 3.467 \times 10^{-2} \mathrm{GeV}^{-1}$ & $b_{1}: 4.115 \times 10^{-3} \mathrm{GeV}^{-1}$ & $c_{1}: 7.417 \times 10^{-3} c^{-2}$ \\
$a_{2}:-8.259 \times 10^{-3} \mathrm{GeV}^{-2}$ & $b_{2}:-2.738 \times 10^{-2} \mathrm{GeV}^{-2}$ & $c_{2}: 2.738 \times 10^{-2} \mathrm{GeV}^{-1} c^{-2}$ \\
\hline \hline
\end{tabular}

Figure 3.17: Two-dimensional distributions of (a) $\log \left(\mathcal{L}_{e} / \mathcal{L}_{\mu}\right)$, (b) $\log \left(\mathcal{L}_{e} / \mathcal{L}_{\pi^{0}}\right)$, and (c) $M_{\gamma \gamma}$ versus the P-Fitter electron hypothesis fitted energy $E_{e}$ for signal and background events. The $E_{e}$ dependent cuts shown by the continuous line in each plot (detailed on the table) are used to select $\nu_{e}$ CCQE events for the TBL oscillation analysis. Taken from [180].

electron, muon, and $\pi^{0}$, and ratios of the maximum likelihoods provide differentiation between them. Denoting the three likelihoods by $\mathcal{L}_{e}, \mathcal{L}_{\mu}$, and $\mathcal{L}_{\pi^{0}}$ respectively, two particle identification discriminants defined by $\log \left(\mathcal{L}_{e} / \mathcal{L}_{\mu}\right)$ and $\log \left(\mathcal{L}_{e} / \mathcal{L}_{\pi^{0}}\right)$ are formed with the sign convention that electron like events have more positive values than $\mu$-like or $\pi^{0}$-like events. Events are also fit under the $\pi^{0}$ hypothesis without a $\pi^{0}$ mass constraint enforced by the default $\pi^{0}$ hypothesis fit. The resulting fitted mass $M_{\gamma \gamma}$ is also used as a discriminant to select or reject $\pi^{0}$ events. Patterson [180] calculated the optimal cut values on $\log \left(\mathcal{L}_{e} / \mathcal{L}_{\mu}\right), \log \left(\mathcal{L}_{e} / \mathcal{L}_{\pi^{0}}\right)$, and $M_{\gamma \gamma}$ as a function of the 
fitted event energy ${ }^{13}$ to maximize the $\nu_{e}$ CCQE selection and minimize the $\nu_{\mu}$ and $\pi^{0}$ misidentification backgrounds. Two dimensional distributions of the three discriminants versus the fitted energy of the event are shown in Figure 3.17. The black curves represent the cut values calculated by Patterson to optimize the signal/background separation. We refer to these cuts as the TBL selection cuts throughout the rest of this thesis. These same cuts were used to produce the first MiniBooNE result in Ref. [190] where different techniques ${ }^{14}$ from those presented in this thesis were used to apply data-driven corrections to the background predictions and to constrain the effect of systematic uncertainties, as will be described in Section 4.1.

\subsubsection{Boosted Decision Tree (BDT) particle identification}

A boosted decision tree machine learning algorithm [193] based on 172 input reconstructed variables from the S-Fitter was used to form a discriminator to separate signal-like events ( $\nu_{e}$ CCQE interactions) from all sources of background. The list of possible inputs to the BDT algorithm was assembled over several years by the MiniBooNE Algorithms group [196]. Many of the variables were determined by specifically considering the physical effects which may separate different types of events, while others were formed by combining both charge and time information, and finding which combinations had more discriminating power. A number of the variables depend directly or indirectly on geometric or kinematic quantities such as the event energy and radial position, introducing correlations between them.

\section{Selecting the input variables}

The input variables were selected from an initial pool of $\sim 1000$ reconstructed vari-

\footnotetext{
13 This is the energy under the P-Fitter electron hypothesis $E_{e}$.

14 These have been described in full detail in [180].
} 
ables of a wide variety. Certain classes of variables are obtained by dividing the tank into 10 equal $\Delta \cos \theta$ intervals around the reconstructed track, where $\theta$ is the photon direction from the track $\mathrm{M} \gamma \mathrm{EP}$ with respect to the reconstructed S-Fitter track direction $^{15}$. This is schematically shown in Figure 3.18(a). For some other variables, the reconstructed track length was divided into 10 track length bins to form 10 rings with the Cherenkov angle of the track, as shown in Figure 3.18(b), to look at information in rings of different sharpness (thin or broad). In either case, the set of PMTs that fall in the angular interval of interest is considered. Intervals of corrected time were also used to define variables capable of distinguishing events by their time structure. This binning scheme was used for a number of observables such as, for example, the total event charge, PMT hit times, or the contribution to the S-Fitter reconstruction likelihoods (time, charge or total) under the electron or $\pi^{0}$ fit hypotheses. The initial input variables can be grouped into five major categories.

1. Reconstructed physical/geometric observables: These include reconstructed quantities from the S-Fitter like the visible energy from the Fast, Full and $\pi^{0}$ fits, the $\pi^{0}$ mass, track length and angle with respect to the beam, fraction of Cherenkov and scintillation light, fraction of light from the photons in the $\pi^{0} \rightarrow \gamma \gamma$ fit, distance of $\mathrm{M} \gamma \mathrm{EP}$ from the wall, among others.

2. Time-related variables: Include, for example, time likelihood values from the S-Fitter to electron or $\pi^{0}$ hypotheses from all PMTs, and from PMTs in $\cos \theta$ and ring sharpness bins. Also likelihood ratios and products between different $\cos \theta$ and sharpness bins are calculated.

3. Charge/Hits-related variables: Include combinations of charge-likelihoods from the S-Fitters similar to those of time-related variables. Also Include ratios

\footnotetext{
${ }^{15}$ No variables from the P-Fitter are used as inputs to the BDT algorithm.
} 


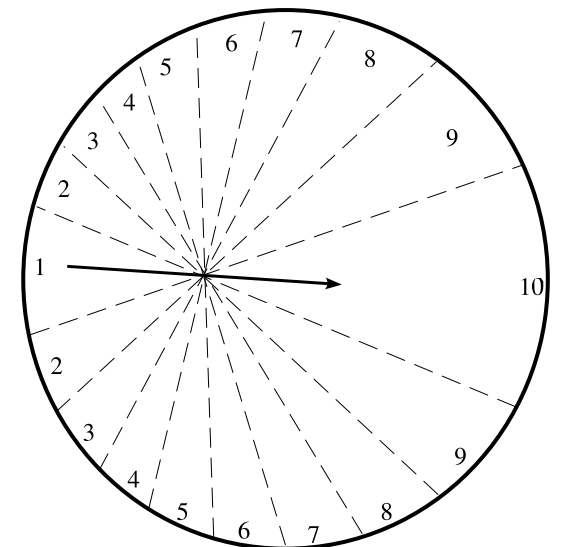

(a) $\cos \theta$ bins

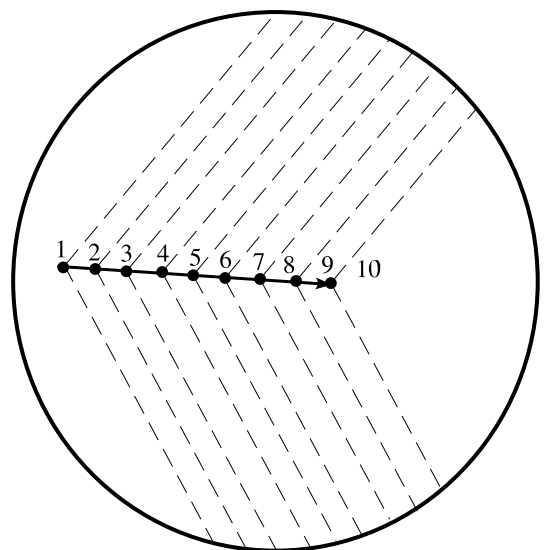

(b) Ring sharpness bins

Figure 3.18: Schematic of $\cos \theta$ and ring sharpness bins (see text) used to define some of the input variables for the BDT algorithm.

of un-hit PMTs to hit PMTs, and ratios of hit PMTs to total PTMs in the various $\cos \theta$ and ring sharpness bins. The Charge likelihood ratios are also separated in those from hit and un-hit PMTs. These also include measured and predicted charges in the PMTs and ratios of these in the various angular and ring sharpness bins.

4. Combinations of Charge and time information: These include charge likelihoods and number of hits in corrected time bins, fraction of prompt and late hits in an event, etc.

5. Auxiliary variables from the minimization: These include the best fit value found by MINUIT in each of the algorithm steps (Full, Flux, $\pi^{0}$ fits), and combinations (sums and differences) of them.

The number of variables was reduced to 172 by requiring: (a) good agreement between data and simulation (differences must lie within systematic uncertainties) in all of the six open boxes in table 3.11, and (b) significantly contribution to the final discriminator (e.g. if a variable is removed, the classifier becomes weaker). A final 
Table 3.11: The Boxes used in the PID input validation of the BDT algorithm.

\begin{tabular}{ll}
\hline \hline Box Name & Dominant event type \\
\hline $\mathrm{CCQE}$ & $\nu_{\mu}$ CCQE interactions \\
$\mathrm{NC} \pi^{0}$ (S-Fitter) & $\mathrm{NC} \pi^{0}$ events \\
$\mathrm{CC} 1 \pi$ & Interactions producing a single $\pi^{+}$or $\pi^{-}$. \\
$\mathrm{NC}$ box & Neutral Current interactions \\
Michel & Michel electron from stopped muons \\
$10 \%$ Box & All event types (satisfies blindness) \\
\hline \hline
\end{tabular}

sub set of 172 variables was used to train the algorithm.

The application of the BDT technique to the MiniBooNE input variables for signal/background separation has been extensively studied [192].

\section{The BDT algorithm}

The goal of the algorithm is to separate signal-like events $\left(\nu_{e}\right.$ CCQE) from all backgrounds using a single PID discriminant variable (called score). Using pure Monte Carlo event samples a collection of $\sim 1000$ decision trees is trained to separate signal from background, with each tree contributing to the calculation of the score of an event. A toy example of a simple decision tree is shown in Figure 3.19, where three variables are used for signal/background separation: PMT hit multiplicity, energy, and radial position.

The algorithm initially assigns an equal weight $W_{i}=1 / N$ to each of the $N$ events in the training sample (signal plus background) and loops over all possible cuts on the 172 input variables to select one that provides the highest separation into its signal and background components. The criterion to select the cut at a given branch is 
based on the calculation of a quantity called the Gini index, defined as

$$
\text { Gini }=\left(\sum_{i} W_{i}\right) P(1-P)
$$

where $P$ is the purity of the separation defined as

$$
P=\frac{\sum_{s} W_{s}}{\sum_{s} W_{s}+\sum_{b} W_{b}}
$$

and the sum $\sum_{s}\left(\sum_{b}\right)$ is over signal (background) events only. The chosen input variable and cut value are chosen as to maximize the difference in Gini index between the parent node and its two daughters:

$$
\text { Criterion }=\text { Gini }_{\text {parent }}-\left(\text { Gini }_{\text {daughter left }}+\text { Gini }_{\text {daughter right }}\right)
$$

Notice that a pure signal or background leaf has $P(1-P)=0$, therefore, a cut that achieves perfect separation maximizes the criterion in Eq.(3.42) in the best possible way.

Once the starting cut for the tree is found, the procedure is repeated by adding more branches to the tree increasing at each step the number of terminal leaves, which is a parameter to be optimized. The BDT algorithm used for the oscillation analysis used trees with a maximum of 45 leaves. When the tree reaches the desired size the purity $P$ of its terminal leaves is evaluated and those with $P>1 / 2$ are labeled signal (S) leaves, and the rest are labeled background (B) leaves. Next, the weight of all events in the training sample that were misclassified ${ }^{16}$ is increased (boosted). A second tree is built using the new weights to define the Gini index and purity of its nodes. When it is finished, the weights of misclassified events are boosted again, and the procedure is repeated until $N_{\text {tree }} \sim \mathcal{O}(1000)$ trees are constructed.

\footnotetext{
${ }^{16}$ Correctly classified events are signal events that land in a $\mathrm{S}$ leaf and background events that land in a B leaf. Signal events landing in a B leaf and background events landing in a S leaf are called misclassified events.
} 


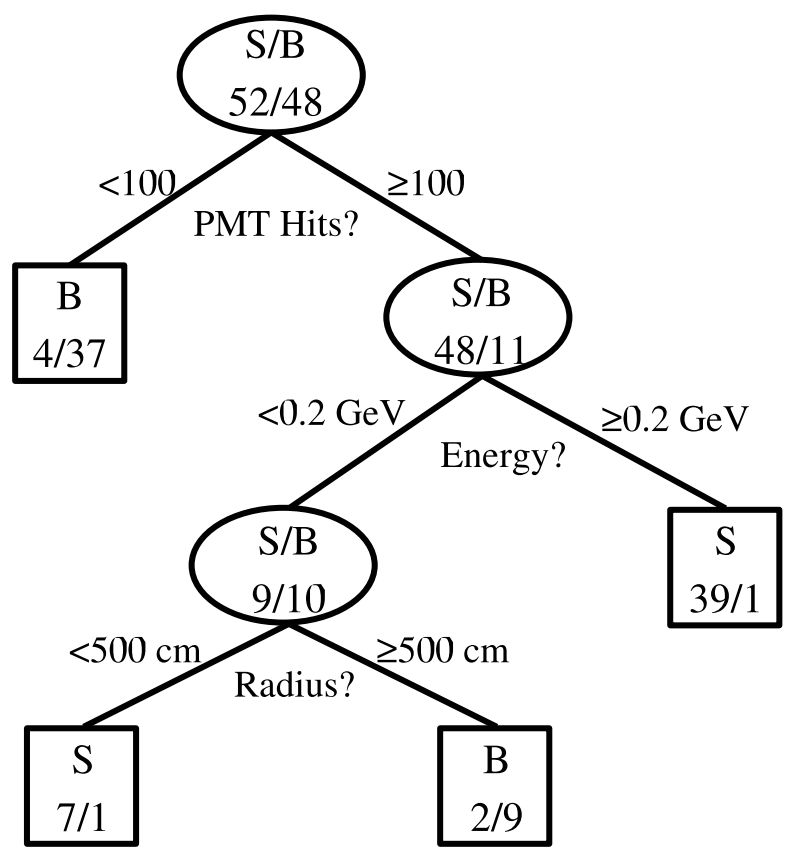

Figure 3.19: Schematic of a decision tree with three nodes (ellipses) and four leaves (boxes). Cuts are applied at each node. If signal (background) events dominate, a leaf is called signal (background) leaf. S stands for signal and B stands for background. Taken from [192]. 
The increase of the weight of misclassified events that was used corresponds to the variety of boosting algorithms known as AdaBoost [193] in which for the $m$ th tree in the sequence one defines

$$
\begin{aligned}
\operatorname{err}_{m} & =\frac{\sum_{i=1}^{N} W_{i} \times I_{i}^{m}}{\sum_{i=1}^{N} W_{i}} \\
\alpha_{m} & =0.5 \times \ln \left(\frac{1-\operatorname{err}_{m}}{\operatorname{err}_{m}}\right)
\end{aligned}
$$

where $I_{i}^{m}=1$ if event $i$ is misclassified by tree $m$, and zero otherwise. In Eq.(3.43) the quantity $\operatorname{err}_{m}$ represents the rate of misclassified events whose weight will be boosted by an amount determined by the quantity $\alpha_{m}$. A typical progression of $\operatorname{err}_{m}$ and $\alpha_{m}$ as a function of the number of trees is shown in Figure 3.20 showing that after $\sim 500$ trees the algorithms is well stabilized. The AdaBoost prescription to set the weights

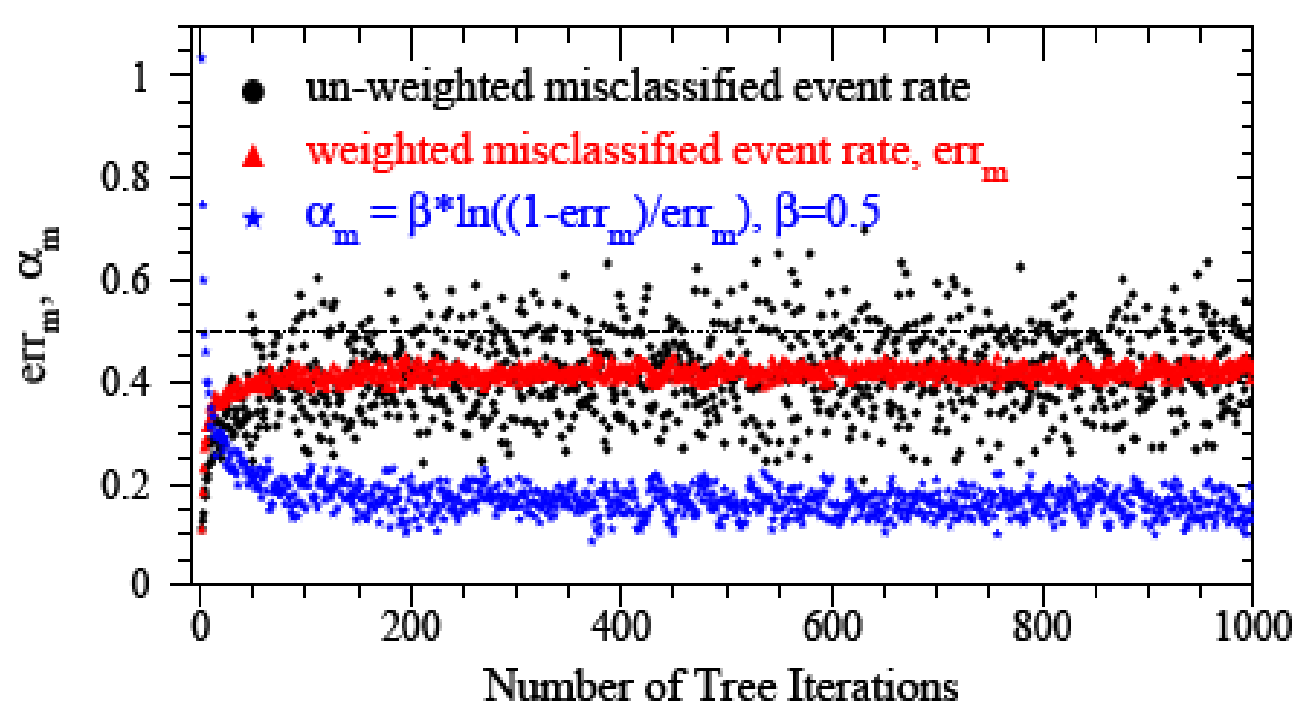

Figure 3.20: AdaBoost progression of the misclassified event rate (red triangles) and weight factor $\alpha_{m}$ (blue stars) in Eq.(3.43) as a function of the number of trees. For comparison the un-weighted number of misclassified events is shown as the black points. Taken from [192].

of the events for the calculation of the $(m+1)$ th tree in the sequence is:

$$
W_{i} \rightarrow W_{i} \times \exp \left(\alpha_{m} I_{i}^{m}\right), \quad i=1, \ldots, N,
$$


Each tree in the sequence assigns a partial score $T_{m}(x)=1\left(T_{m}(x)=-1\right)$ to event $x$ when it lands in a S (B) leaf. The final score is defined as

$$
T(x)=\sum_{i=1}^{N_{\text {tree }}} \alpha_{m} T_{m}(x)
$$

which is the weighted sum of the partial scores from all the trees. After training the algorithm ${ }^{17}$ the set of trees $T_{m}$ and weights $\alpha_{m}$ are fixed and are ready to be applied to event from a test sample, from which the performance of the algorithm is evaluated.

\subsubsection{The $\nu_{e}$ selection of the BDT and TBL analyses}

As we have seen, the TBL and BDT particle identification techniques are each based in one of the two reconstruction algorithms described in Section 3.4:

- BDT PID: based on S-Fitter reconstruction.

- TBL PID: based on P-Fitters reconstruction.

Keeping this separation was motivated by the idea to perform two oscillation analyses that would be complimentary to each other, the most powerful of which would provide the main result of the experiment, while the second would be a powerful cross check. As described in [190] the oscillation analysis based on the TBL reconstruction proved to yield the highest sensitivity to oscillations, and on this basis was selected as the primary analysis.

17 To increase the separation power the algorithm the cascade-training technique described in [194] was implemented. 


\section{Two analyses}

We henceforth refer to the two possible ways to proceed towards an oscillation result as the TBL analysis and BDT analysis. In both cases a fit to the reconstructed neutrino energy distribution ${ }^{18}$ will be used to interpret any excess of events as an LSND like oscillation signal. We will denote the neutrino energy of the TBL analysis by $E_{\nu}^{Q E}-T B L$ which is calculated with Eq.(3.17) using P-Fitter quantities, and that of the BDT analysis by $E_{\nu}^{Q E}-B D T$, which besides being based on the S-Fitter reconstruction, is calibrated as described in Appendix A. Both analyses share a few common cuts to select $\nu_{e}$ candidate events but differ in the more powerful part of their selection criteria. The common cuts used by both, the BDT and the TBL analyses are:

\section{- Only one sub-event is present:}

Removes events with muon decays. Clean $\nu_{e}$ CCQE interactions should produce a single electron track.

- $N_{\text {veto }}<6$ hits in the veto:

Removes cosmic rays and other activity from the outside of the tank that penetrates the veto.

- $N_{\text {tank }}>200$ hits in the main tank:

Eliminates Michel electrons from muons that entered the tank before the DAQ window was open.

Subsequent cuts are algorithm specific, and are described below.

\section{TBL-specific $\nu_{e}$ selection}

\footnotetext{
${ }^{18}$ Reconstructed under the hypothesis of a $\nu_{e}$ CCQE interaction.
} 
We will denote the fiducial volume cuts used in the TBL analysis as $R_{v t x}<500 \mathrm{~cm}$ for the requirement that the reconstructed event vertex lies within the sphere with 500 $\mathrm{cm}$ radius, and $R_{\text {end }}<488 \mathrm{~cm}$ the requirement that the end point of the reconstructed track lies within $488 \mathrm{~cm}$ from the tank center. The TBL analysis uses an additional low visible energy cut $E_{e}<140 \mathrm{MeV}$ to avoid mis-reconstruction of the neutrino energy and reduce low energy backgrounds. Both the visible energy on which this cut is based, and the reconstructed neutrino energy used in the TBL oscillation analysis are calculated under the assumption that there is only one track in the event. $\nu_{e}$ candidate events for the TBL analysis are required to satisfy the cuts described in Figure 3.17. The signal efficiency of the TBL analysis cuts is shown in Figure 3.21.

\section{BDT-specific $\nu_{e}$ selection}

The BDT analysis uses a different fiducial volume cut, based on the S-Fitter algorithm. We will denote the fiducial volume cut in this analysis as $R_{T C}<500 \mathrm{~cm}$, where the subscript $T C$ refers to the track center ${ }^{19}$. The $\nu_{e}$ CCQE candidates of the BDT analysis are required to satisfy the energy dependent BDT score cut shown by the solid black line in Figure 3.22. The cut values in each energy bin were optimized to yield the highest sensitivity to oscillations when considering the dominant source of systematic uncertainty for the BDT analysis.

Optimization of the BDT score cut:

A MINUIT [195] minimization routine was used to find the optimal value of the BDT score output as a function of the $E_{\nu}^{Q E}-B D T$ reconstructed neutrino energy to maximize the sensitivity to oscillations simultaneously at three values of the oscillation parameter $\Delta m^{2}$ in Eq.(1.12).

\footnotetext{
${ }^{19}$ The mean photon emission point $\mathrm{M} \gamma \mathrm{EP}$ described in Section 3.4.1
} 

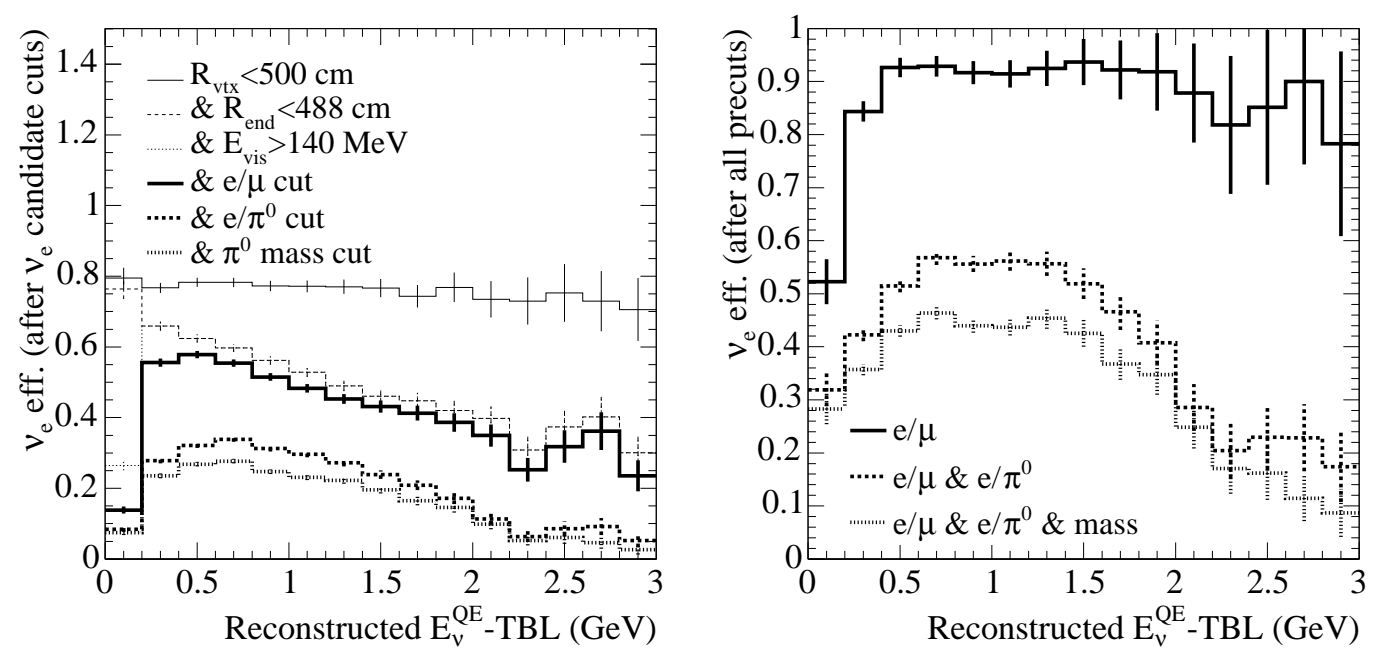

(a) $\nu_{e}$ efficiency (all interactions)
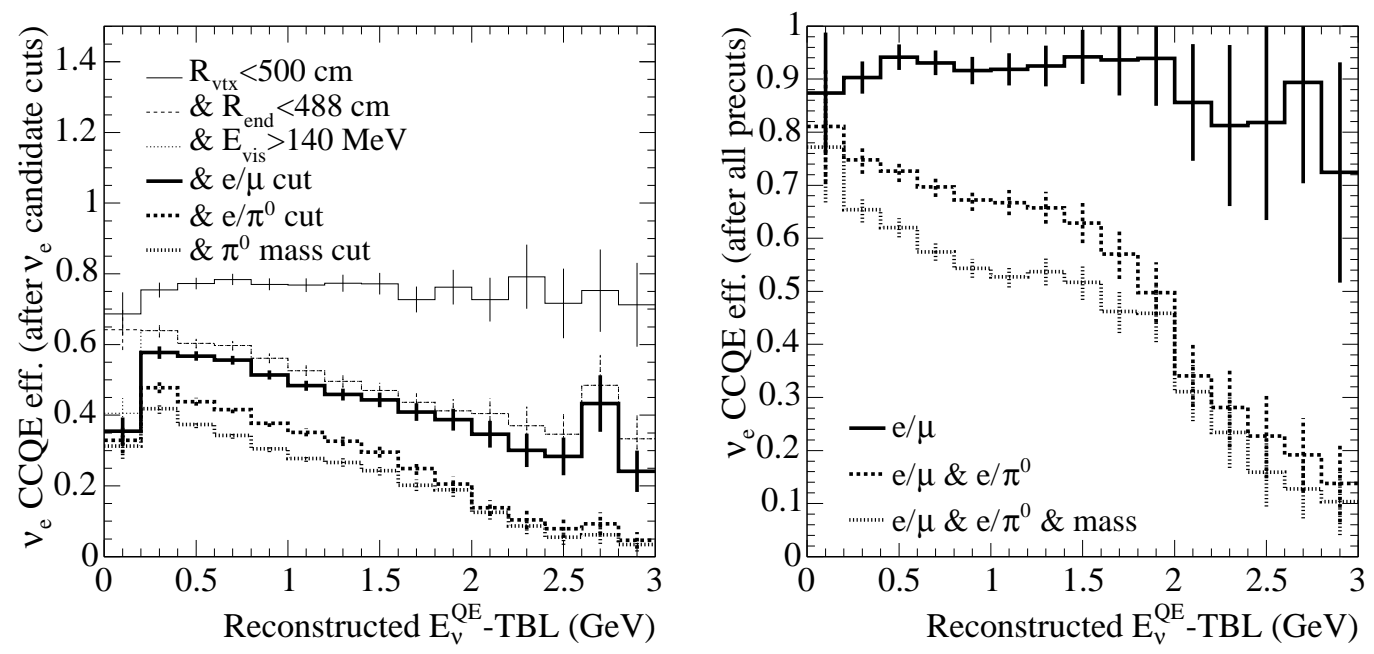

(b) $\nu_{e}$ efficiency (CCQE interactions).

Figure 3.21: $\nu_{e}$ efficiency of the TBL selection cuts as a function of the reconstructed neutrino energy $E_{\nu}^{Q E}-T B L$. Left: Efficiency relative to neutrino candidate cuts $\left(N_{\operatorname{tank}}>\right.$ $200 \& N_{\text {veto }}<6 \&$ only 1 sub-event); Right: Efficiency of the $e / \mu, e / \pi^{0}$, and $\pi^{0}$ mass cuts of Section 3.5.1, relative to all TBL specific precuts. 


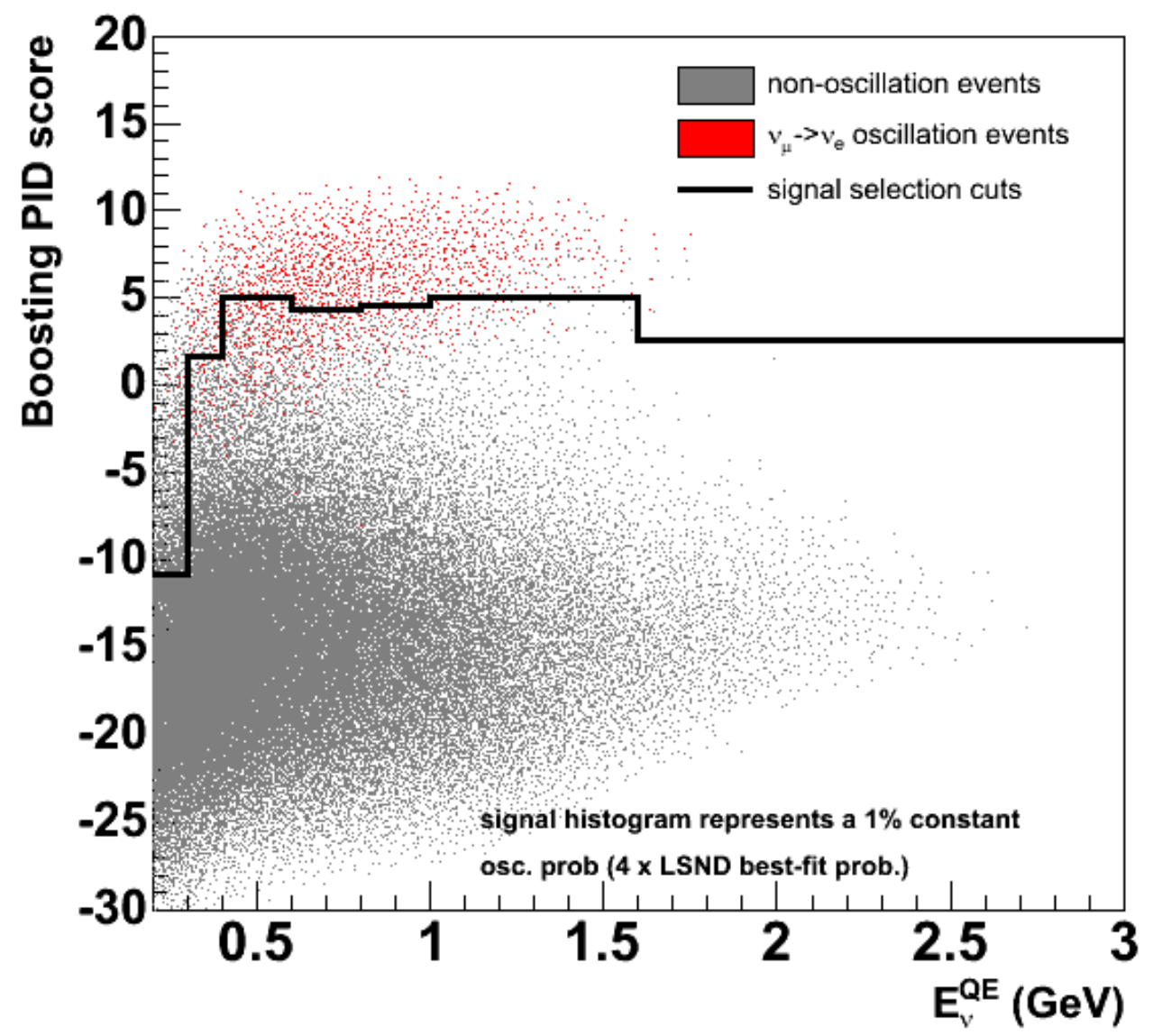

Figure 3.22: The energy-dependent BDT score cut (solid line) used in the $\nu_{e}$ CCQE selection of the BDT analysis. The plotted energy is $E_{\nu}^{Q E}-B D T$. The distribution of BDT score values for $\nu_{e}$ CCQE events (red points) and all backgrounds (gray points) is also shown. The BTD cut value at each bin was optimized to yield the maximum sensitivity to oscillations. 

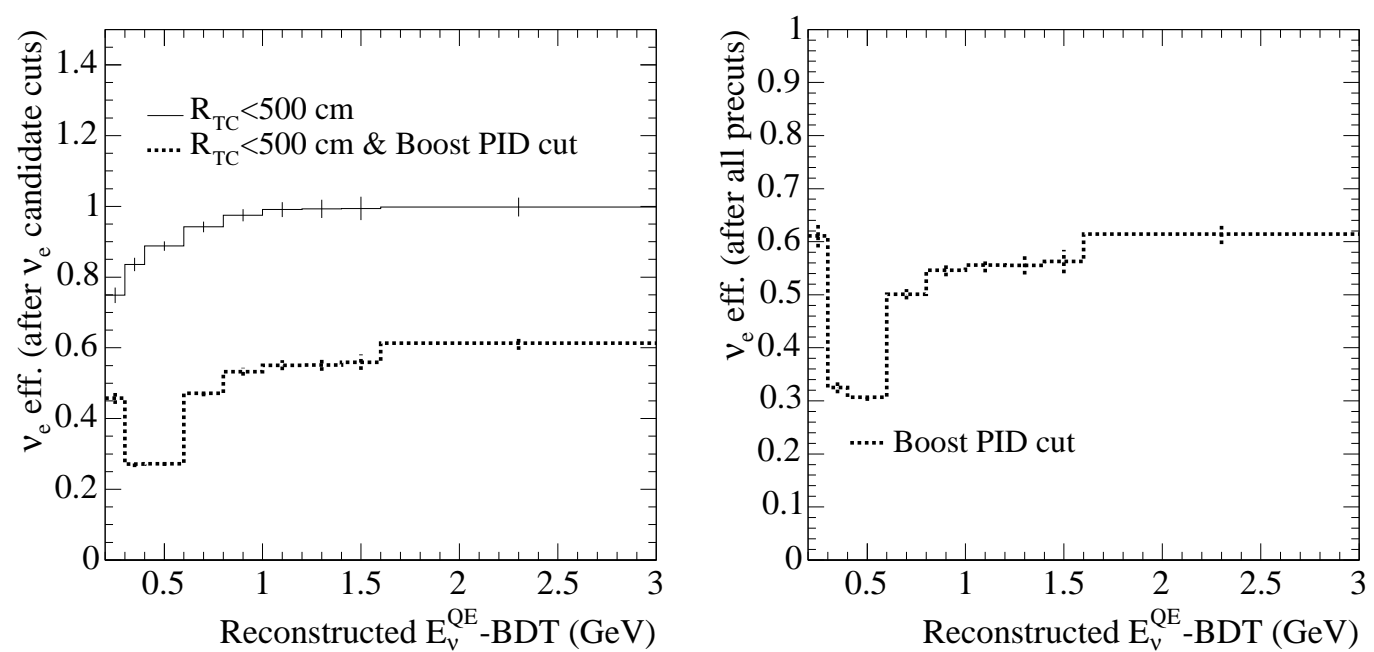

(a) $\nu_{e}$ efficiency (all interactions).
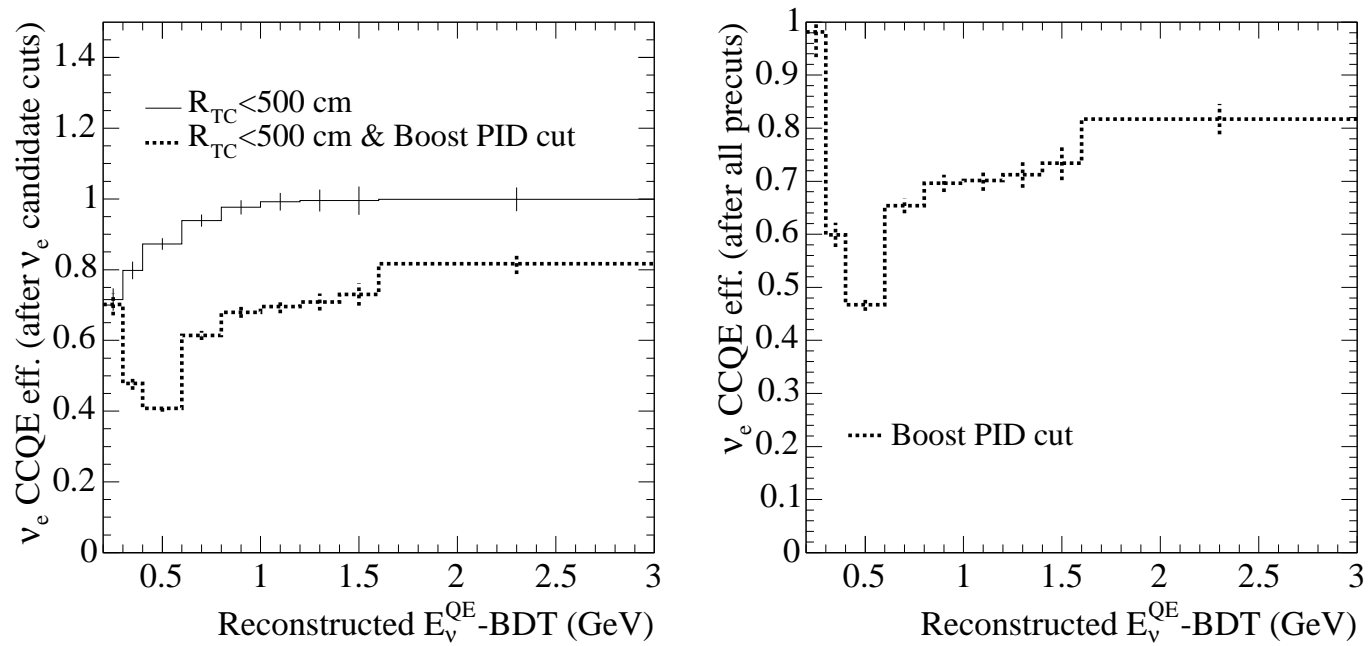

(b) $\nu_{e}$ efficiency (CCQE interactions)

Figure 3.23: $\nu_{e}$ efficiency of the BDT selection cuts as a function of the reconstructed neutrino energy $E_{\nu}^{Q E}-B D T$. Left: Efficiency relative to neutrino candidate cuts $\left(N_{\operatorname{tank}}>\right.$ $200 \& N_{\text {veto }}<6 \&$ only 1 sub-event); Right: Efficiency of the BDT cuts of Section 3.5.2 relative to all BDT specific precuts. 
It is interesting to note that the efficiency of the BDT $R_{T C}<500 \mathrm{~cm}$ cut as a function of energy has a different behavior when compared to the efficiency of the corresponding TBL fiducial volume cut of $R_{v t x}<500 \mathrm{~cm}$, as can be seen by comparing the top-left plots in Figures 3.21 and 3.23. While the $\sim 75 \%$ efficiency of the $R_{v t x}<500 \mathrm{~cm}$ cut in the TBL analysis is flat with the reconstructed energy, the efficiency of the $R_{T C}<500 \mathrm{~cm}$ of the BDT analysis is $\sim 75 \%$ only for the lowest energy tracks (also the shortest) and raises to $100 \%$ for energies above $\sim 1 \mathrm{GeV}$. The reason of this difference is that contained events with high enough deposited energy $(>1 \mathrm{GeV})$ will produce sufficiently long tracks $(\gtrsim 700 \mathrm{~cm}$ ) always having their reconstructed track centers well within the tank wall and pass the BDT fiducial volume cut, while the reconstructed track vertices used for the TBL cut are homogeneously distributed inside the tank, with approximately $75 \%$ of them lying more than $\sim 50 \mathrm{~cm}$ away from the PMT faces, beyond which only a few contained event vertices are reconstructed ${ }^{20}$.

\subsubsection{The $\nu_{\mu}$ CCQE sample}

The $\nu_{\mu}$ CCQE sample used in this thesis is described in [191], and was isolated by requiring the detection of the primary muon and the associated decay electron $\left(\nu_{\mu}+n \rightarrow \mu^{-}+p, \quad \mu^{-} \rightarrow e^{-}+\nu_{\mu}+\bar{\nu}_{e}\right)$. Timing information from the PMTs allows one to separate the light produced by the muon in the initial neutrino interaction (first "sub-event") from the light produced by the decay electron (second "sub-event"). The position and momentum of the primary particles in each sub-event are reconstructed with the S-Fitter algorithm. We require that the first sub-event (the neutrino interaction) satisfy the S-Fitter based cut $R_{T C}<500 \mathrm{~cm}$, fire $<6$ veto-PMTs to ensure its containment, and > 200 tank-PMTs to avoid electrons from cosmic ray muon

\footnotetext{
${ }^{20}$ The sphere tangent to the PMT surfaces has radius of $550 \mathrm{~cm}$, therefore: $(500 \mathrm{~cm} / 550 \mathrm{~cm})^{3} \sim$ 0.75
} 
decays. The second sub-event (the $\mu^{-}$decay electron) must fire $<6$ veto-PMTs and $<200$ tank-PMTs. Subsequent cuts specifically select $\nu_{\mu}$ CCQE events, discriminating against neutrino induced single pion $\left(\mathrm{CC} 1 \pi^{+}\right)$backgrounds. First, events must contain exactly two sub-events. Second, the distance between the electron vertex and the muon track endpoint must be less than $100 \mathrm{~cm}$, to ensure that the decay electron is associated with the muon track.

The previous selection yields a total of 193,709 events from the $5.58 \times 10^{20}$ protons on target (POT) accumulated in neutrino mode (positive horn polarity). These cuts are estimated to be $35 \%$ efficient and to have a CCQE purity of $74 \%$.

The total number of $\nu_{\mu}$ CCQE events in the data was found to be $1.22 \pm 0.27$ times higher than that in the simulation This normalization correction is well within the total uncertainties and assumed to be due to a normalization error on the number of $\pi^{+}$decaying into neutrinos in the detector acceptance. The effect of this normalization correction was propagated to the simulation by scaling all events originating from decaying $\pi^{+}$by this factor. 


\section{Chapter 4}

\section{Oscillation analysis with two $\nu_{e}$ candidate samples}

The primary goal of the experiment is to test the scenario of 2-neutrino oscillations of the type $\nu_{\mu} \rightarrow \nu_{e}$, and specifically, those described by the parameters allowed by the LSND result. MiniBooNE can make use of the large flux of $\nu_{\mu}$ 's to normalize its predicted number of background events to this signal, and use this information to constrain the magnitude of the uncertainties affecting the prediction of $\nu_{e}$ events. The approach followed in this thesis is similar to that presented in [181], but has been extended to deal with two input $\nu_{e}$ event samples plus one $\nu_{\mu}$ sample, and restricted to a $\nu_{e}$ appearance search. The procedure has been applied to the actual MiniBooNE data set for $(5.58 \pm 0.12) \times 10^{20}$ POT collected between the years 2002 and 2006 .

\subsection{Analysis overview}

In its first publication [190] the MiniBooNE collaboration reported on the agreement of the observed number of $\nu_{e}$-induced events with background expectations in the 
absence of $\nu_{\mu} \rightarrow \nu_{e}$ appearance-only oscillations of the LSND [37, 38, 39, 40] type in the range from $475 \mathrm{MeV}$ to $3000 \mathrm{MeV}$ of reconstructed neutrino energy. The $\nu_{e}$ sample used in that measurement was isolated using the particle identification (PID) method based on likelihood ratios described in Section 3.5.1. Following the nomenclature of Section 3.5.3, we refer to this result as the track-based likelihood (TBL) analysis.

The TBL analysis in the first MiniBooNE publication [190] used a high statistics sample of $\nu_{\mu}$ charged-current quasi-elastic (CCQE) events to correct the number of expected background events to the $\nu_{\mu} \rightarrow \nu_{e}$ oscillation search, and to reduce the magnitude of the systematic uncertainties associated with these predictions. The corrected predictions and reduced errors were then used in a fit of the reconstructed neutrino energy distribution under the two-neutrino appearance-only oscillations hypothesis ${ }^{1}$. In the same publication the result of a cross-check analysis supporting the conclusion of the TBL analysis was also presented. This is the BDT oscillation analysis, whose $\nu_{e}$ selection criteria was also described in Section 3.5.3. The BDT analysis, in contrast, used the technique of introducing the $\nu_{\mu}$ sample into the $\chi^{2}$ minimization of the oscillations fit. The reduction of systematic uncertainties is accomplished by the presence of a penalty coming from the correlations between the reconstructed neutrino energy bins of the $\nu_{\mu}$ and $\nu_{e}$ samples. The two analyses make use of distinct but complementary $\nu_{e}$ candidate samples. While there is large overlap of events, there are significant differences that make attractive their use in a combined analysis, which is the purpose of this thesis.

In Section 4.2, we describe the combined $\nu_{\mu} / \nu_{e}$ fitting technique, the estimation of systematic errors, and its implementation to calculate the limit to oscillations from the BDT analysis appearing in Ref.[190]. The BDT analysis results are reviewed in Section 4.3. In Section 4.4 we show that the combined fit technique used with the

\footnotetext{
${ }^{1}$ Details of the constraining procedure have been given elsewhere by Patterson [180].
} 
TBL selection yields an equivalent systematic error constraint as that achieved by the alternative technique used in Ref.[190], leading to a comparable sensitivity and limit to oscillations. In Section 4.5 we extend the combined fit technique to use the three samples (BDT $\nu_{e}$, TBL $\nu_{e}$ and $\nu_{\mu}$ CCQE) together while taking care of the overlap of events between the two $\nu_{e}$ samples and the effect of the correlations between them in the estimate of systematic uncertainties. We show that the sensitivity to oscillations so obtained is increased. The final results are presented in chapter 5 .

We would like to emphasize that, as in Ref.[190], the present analysis of the MiniBooNE data is performed within a two-neutrino appearance-only $\nu_{\mu} \rightarrow \nu_{e}$ model, and that no other effects beyond the standard model are assumed.

\section{Energy distributions}

Each of the two analyses performs a fit to the reconstructed neutrino energy distribution of $\nu_{e}$ candidate events calculated under the hypothesis of a CCQE interaction with a neutron at rest, in which the energy and scattering angle of the outgoing particle track fully determine the neutrino energy when its incoming direction is known. As mentioned in Section 3.5.3 the neutrino energy so obtained is denoted $E_{\nu}^{Q E}$-BDT and $E_{\nu}^{Q E}-\mathrm{TBL}$ in each case. The two energy estimators will in general be different even for events that are present in both $\nu_{e}$ candidate samples.

The reconstructed neutrino energy distributions of the $\nu_{\mu}$ CCQE sample and the two $\nu_{e}$ candidate samples (BDT and TBL cuts), are shown in Figure 4.1. The Monte Carlo prediction has been adjusted by weighting the contribution of all events from a decaying $\pi^{+}$by a factor of 1.22 (see Section 3.5.4). We emphasize that the $\nu_{\mu}$ CCQE sample in the top plot in Figure 4.1 used the energy estimator based on the S-Fitter and the $\nu_{\mu}$ CCQE calibration of Appendix $\mathrm{A}^{2}$. The composition of the samples as

\footnotetext{
${ }^{2}$ The $\nu_{\mu}$ CCQE sample used in Ref.[190] had slightly different selection criteria and an energy
} 

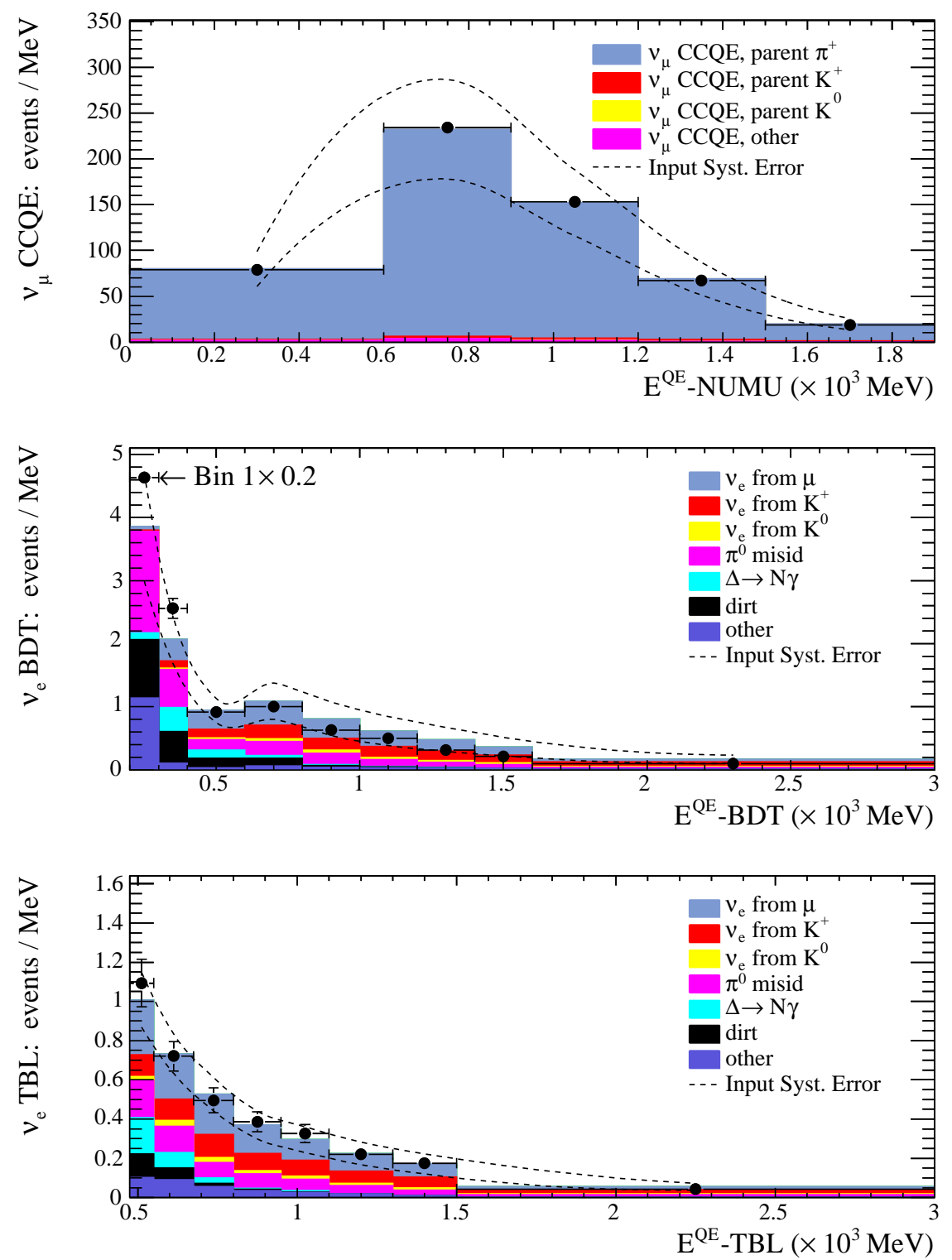

Figure 4.1: Reconstructed neutrino energy distributions for the $\nu_{\mu}$ CCQE sample (top), the BDT $\nu_{e}$ candidate sample (middle), and the TBL $\nu_{e}$ candidate sample (bottom). The dashed curves represent the unconstrained total systematic uncertainties as calculated from the procedure described in the text. For display purposes, the first bin in the BDT distribution has been scaled to $20 \%$ of its value. 


\begin{tabular}{lcc}
\hline \hline Process & Number of events \pm unconstrained uncertainty \\
\hline & BDT & TBL \\
$E_{\nu}^{Q E}$ range $(\mathrm{MeV})$ & {$[300,1600]$} & {$[475,1250]$} \\
\hline $\mathrm{NC} \pi^{0}$ & $223 \pm 89 \pm 20$ \\
$\mathrm{NC} \Delta \rightarrow N \gamma$ & $78 \pm 20$ & $29 \pm 6$ \\
Dirt & $115 \pm 18$ & $19 \pm 3$ \\
$\nu_{e}$ from $\mu$ decay & $342 \pm 119$ & $127 \pm 34$ \\
$\nu_{e}$ from $K^{+}$decay & $207 \pm 79$ & $71 \pm 21$ \\
$\nu_{e}$ from $K_{L}^{0}$ decay & $44 \pm 24$ & $14 \pm 5$ \\
Other & $54 \pm 32$ & $38 \pm 18$ \\
Total Background & $1066 \pm 266$ & $368 \pm 52$ \\
Osc [sin $\left.2 \theta=0.004, \Delta m^{2}=1 \mathrm{eV}^{2}\right]$ & $302 \pm 93$ & $133 \pm 32$ \\
\hline \hline
\end{tabular}

Table 4.1: The expected number of events passing the $\nu_{e}$ candidate selection cuts in both analyses. The uncertainties correspond to the total unconstrained error estimates consistent with the error bands in Figure 4.1. The last row shows the expected number of events for an LSND type $\nu_{\mu} \rightarrow \nu_{e}$ oscillation with the parameters shown.

predicted by the Monte Carlo is displayed in the figure. The dashed lines represent the total systematic uncertainty expected on the predicted number of events, and was calculated with the procedure described in the next section. The number of events in these distributions correspond to the full POT in the neutrino mode run. Note that the $\nu_{\mu} \mathrm{CCQE}$ distribution is truncated at $1.9 \mathrm{GeV}$, the total number of $\nu_{\mu} \mathrm{CCQE}$ events from the data in this range is 190,917.

The numbers of events passing the two separate $\nu_{e}$ candidate selection cuts are estimator based on the P-Fitter reconstruction For details on this sample see [180]. 
shown in Table 4.1. The uncertainties in the table will be reduced by using the high statistics of the observed $\nu_{\mu}$ CCQE sample. There is significant overlap in the events passing both $\nu_{e}$ selection criteria. If we want to benefit from the total number of events present in both samples it is necessary to take into account the statistical correlations that the overlap creates.

\subsection{The combined $\nu_{\mu} / \nu_{e}$ fit technique used in the BDT analysis.}

The result from the BDT analysis in Ref.[190] makes use of a sample of candidate $\nu_{e}$ events selected with the energy dependent cut in Figure 3.22, as well as the high statistics sample of $\nu_{\mu}$ CCQE events described earlier. The $\nu_{e}$ candidate and $\nu_{\mu}$ CCQE samples are used together in the definition of a $\chi^{2}$ statistic that is used to fit a two-neutrino oscillations hypothesis to the data. In the fit only the number of events in the $\nu_{e}$ sample is affected by the oscillation parameters $\sin ^{2} 2 \theta$ and $\Delta m^{2}$. We used a two-dimensional (2D) $\chi^{2}$ minimization technique to find the best fit oscillation parameters describing the data. A one-dimensional (1D) raster-scan fit for $\sin ^{2} 2 \theta$ at each $\Delta m^{2}$ value was also performed and shown to find the same best fit point as the 2D fit. We used the results from the 1D method to report limits on oscillations.

\subsubsection{Definition of $\chi^{2}$}

A $\chi^{2}$ statistic is calculated comparing the observed energy distributions for the $\nu_{e}$ and $\nu_{\mu}$ samples with the predictions for a given point in the oscillations parameter 
space. The details of the $\chi^{2}$ definition are given in Eq.(4.1) below.

$$
\begin{gathered}
\chi^{2}\left(\Delta m^{2}, \sin ^{2} 2 \theta\right)=\sum_{i, j}^{N_{e}+N_{\mu}}\left(m_{i}-t_{i}\right) M_{i j}^{-1}\left(m_{j}-t_{j}\right) \\
\nu_{e} \text { candidate bins: } i=1, \ldots, N_{e} \\
\nu_{\mu} \text { candidate bins: } i=N_{e}+1, \ldots, N_{e}+N_{\mu} \\
t_{i}\left(\sin ^{2} 2 \theta, \Delta m^{2} ; f_{\pi}, f_{K}\right) \\
m_{i}=\text { Number of observed data events in bin } i, \\
t_{i}=\text { Number of predicted events in bin } i, \\
M_{i j}^{-1}=\text { Inverse of the covariance matrix, } \\
f_{\pi}\left(f_{K}\right)=\text { pion (kaon) normalization factor. }
\end{gathered}
$$

The predicted number if events $t_{i}$ depends on the oscillation parameters $\sin ^{2} 2 \theta$ and $\Delta m^{2}$, as well as in two normalization factors $f_{\pi}$ and $f_{K}$, which are fixed during the minimization $^{3}$. The indices $i, j$ run over the bins of both distributions $\left(N_{e} \nu_{e}\right.$ bins and $N_{\mu} \nu_{\mu}$ bins) and the number of predicted events for the $\nu_{\mu}$ CCQE sample ( $t_{i}$ for $\left.i=N_{e}+1, \ldots, N_{e}+N_{\mu}\right)$ does not vary with the oscillation parameters. The matrix $M_{i j}$ is the total covariance matrix including all sources of uncertainty, statistical and systematic, and contains the correlations between the $\nu_{e}$ and $\nu_{\mu}$ bins.

\section{Calculating the predicted number of events $t_{i}$}

The predicted number of events $t_{i}$ is a combination of background and signal event estimates from various sources. The event estimates are obtained from the MiniBooNE Monte Carlo separately for events from pion and kaon parent, and using a sample of $100 \% \nu_{\mu} \rightarrow \nu_{e}$ transmutated events ${ }^{4}$ modulated by the oscillation probability Eq.(1.12) for the signal component. Events from external interactions (section

\footnotetext{
${ }^{3}$ We set $N_{\pi}=1.22$ to account for the normalization difference described in Section 3.5.4 and $N_{K}=1.0$.

${ }^{4}$ These are $\nu_{e}$ events with a $\nu_{\mu}$ flux.
} 
3.3), and $\mathrm{NC} \pi^{0}$ interactions (section 3.2.2) are not affected by the normalization factors $f_{\pi}$ and $f_{K}$, since their contributions are set from the observed rates of these events. The various contributions to the predicted number of events in $\nu_{e}$ and $\nu_{\mu}$ bins is shown in

$$
\begin{aligned}
t_{i}^{\nu_{e}} & =f_{\pi} \times \mathrm{MC}\left[\nu_{e} \text { cuts }- \text { no } \pi^{0}\right]_{i}^{\pi^{+}} \text {parent }+f_{K} \times \mathrm{MC}\left[\nu_{e} \text { cuts }- \text { no } \pi^{0}\right]_{i}^{K^{+}} \text {parent } \\
& +\mathrm{NC} \pi^{0}\left[\nu_{e} \text { cuts }\right]_{i}+\operatorname{Dirt}\left[\nu_{e} \text { cuts }\right]_{i}+\text { Other }\left[\nu_{e} \text { cuts }\right]_{i} \\
& +P\left(\sin ^{2} 2 \theta, \Delta m^{2}\right) \times\left(f_{\pi} \times \mathrm{FO}\left[\nu_{e} \text { cuts }\right]_{i}^{\pi^{+}} \text {parent }+f_{K} \times \mathrm{FO}\left[\nu_{e} \text { cuts }\right]_{i}^{K^{+}} \text {parent }\right) \\
t_{i}^{\nu_{\mu}} & =f_{\pi} \times \mathrm{MC}\left[\nu_{\mu} \text { cuts }- \text { no } \pi^{0}\right]_{i}^{\pi^{+}} \text {parent }+f_{K} \times \mathrm{MC}\left[\nu_{\mu} \text { cuts }- \text { no } \pi^{0}\right]_{i}^{K^{+}} \text {parent } \\
& +\mathrm{NC} \pi^{0}\left[\nu_{\mu} \text { cuts }\right]_{i}+\operatorname{Dirt}\left[\nu_{\mu} \text { cuts }\right]_{i}+\operatorname{Other}\left[\nu_{\mu} \text { cuts }\right]_{i}
\end{aligned}
$$

where $P\left(\sin ^{2} 2 \theta, \Delta m^{2}\right)$ is the oscillation probability Eq.(1.12), and the other quantities are explained below:

$$
\begin{aligned}
& \mathrm{MC}\left[\nu_{e, \mu} \text { cuts }- \text { no } \pi^{0}\right]_{i}^{\pi^{+}} \text {parent }=\text { MC events from } \pi^{+} \text {decay passing the } \nu_{e, \mu} \text { cuts } \\
& \text { excluding NC } \pi^{0} \text { events, in bin } i \\
& \mathrm{MC}\left[\nu_{e, \mu} \text { cuts-no } \pi^{0}\right]_{i}^{K^{+}} \text {parent }=\text { MC events from } K^{+} \text {decay passing the } \nu_{e, \mu} \text { cuts } \\
& \text { excluding NC } \pi^{0} \text { events, in bin } i \text {. } \\
& \mathrm{FO}\left[\nu_{e, \mu} \text { cuts }- \text { no } \pi^{0}\right]_{i}^{\pi^{+}} \text {parent }=100 \%\left(\nu_{\mu} \rightarrow \nu_{e}\right)(\text { Fully Oscillated }) \text { events } \\
& \text { from } \pi^{+} \text {decay passing the } \nu_{e, \mu} \text { cuts, in bin } i \\
& \mathrm{FO}\left[\nu_{e, \mu} \text { cuts }- \text { no } \pi^{0}\right]_{i}^{K^{+}} \text {parent }=100 \%\left(\nu_{\mu} \rightarrow \nu_{e}\right)(\text { Fully Oscillated }) \text { events } \\
& \text { from } K^{+} \text {decay passing the } \nu_{e, \mu} \text { cuts, in bin } i \text {. } \\
& \mathrm{NC} \pi^{0}\left[\nu_{e, \mu} \text { cuts }\right]=\mathrm{NC} \pi^{0} \text { events passing the } \nu_{e, \mu} \text { cuts, in bin } i \\
& \operatorname{Dirt}\left[\nu_{e, \mu} \text { cuts }\right]=\text { Events from external interactions passing } \\
& \text { the } \nu_{e, \mu} \text { cuts, in bin } i \\
& \text { Other }\left[\nu_{e, \mu} \text { cuts }\right]=\text { Events from any other source passing } \\
& \text { the } \nu_{e, \mu} \text { cuts, in bin } i
\end{aligned}
$$




\subsubsection{Systematic Uncertainties}

The systematic errors associated with the neutrino flux prediction, modeling of the detector, and neutrino cross sections were estimated as described in [190]. Flux uncertainties are determined from the uncertainties of particle production measurements described in Section 3.1. The neutrino cross Section systematic uncertainties, described in section 3.2, are determined from MiniBooNE data as well as from external sources, both experimental and theoretical. Finally, the detector model systematic uncertainties described in section 3.3 are determined also from MiniBooNE data.

The covariance matrix $M_{i j}$ in Eq.(4.1) is a sum of matrices from each of the uncertainty sources:

$$
\begin{aligned}
M_{i j}= & M^{\pi^{+} \text {flux }}+M^{\pi^{-} \text {flux }}+M^{K^{+} \text {flux }}+M^{K_{L}^{0} \text { flux }}+ \\
& M^{\text {beam }}+M^{\mathrm{Xsec}}+M^{\mathrm{NC} \pi^{0} \text { yield }}+M^{\text {Dirt }}+M^{\text {O.M. }}
\end{aligned}
$$

which is an $\left(N_{e}+N_{\mu}\right) \times\left(N_{e}+N_{\mu}\right)$ matrix. Each component in Eq.(4.4) is associated with the covariance matrix of a set of underlying simulation parameters, and is obtained by sampling the multivariate Gaussian parameter space implied by this covariance. We will refer to the simulations obtained from such sampling of underlying parameters as multisims.

Whenever possible, multisims were constructed with an event-by-event reweighting mechanism to reduce the needed computation. However, sources of uncertainty such as the modeling of the optical properties of the detecting medium and some electronics processes were not suited to be treated by reweighting of events.

For example, the uncertainties in $M^{\pi^{+}}$flux , associated with the Sanford-Wang parameterization of the $\pi^{+}$production of Section 3.1.1, are obtained by calculating the covariance of the bin contents of $\alpha=1, \ldots, 1000$ simulated distributions (multisims), each obtained by reweighting each event $k$, whose parent meson has momentum and 
scattering angle $\left(p_{k}, \theta_{k}\right)$, with the weight:

$$
w_{k}^{\alpha}=\frac{S W\left(c_{1}^{\alpha}, \ldots, c_{8}^{\alpha} ; p_{k}^{\pi}, \theta_{k}^{\pi}\right)}{S W\left(c_{1}^{0}, \ldots, c_{8}^{0} ; p_{k}^{\pi}, \theta_{k}^{\pi}\right)}
$$

where: $\mathrm{SW}=$ Sanford-Wang function for $p_{k}^{\pi}, \theta_{k}^{\pi}$ with given $c^{\prime}$ s

$$
\begin{aligned}
& c_{n}^{\alpha}=\text { SW parameter } n \text { for multisim } \alpha \\
& c_{n}^{0}=\text { SW parameter } n \text { for standard Monte Carlo. }
\end{aligned}
$$

The optical model (O.M.) errors $M_{i j}^{O . M .}$ are an example where such reweighting cannot be used to determine the effect of a change in underlying parameters on the energy distributions of samples after selection cuts. In this case, OM multisims were produced by generating 66 fully reconstructed hit-level simulations which where then passed through the selection cuts for the various samples. The statistics of the O.M. multisims was chosen to match the normalization of the $\nu_{\mu}$ CCQE data set for the $5.58 \times 10^{20}$ POT. Energy scale errors are contained in the O.M. component.

In our error estimates we have included the effect of electronics modeling of the discriminator threshold levels and the charge-time corrections in the photoelectric response of the PMTs. However, such components can only be studied in discrete variations. We took single excursions and estimated the associated error by the difference between the excursion and the default settings. We term such sources of simulation unisims.

\section{Error matrix calculation}

In practice, we construct an expanded version of the covariance matrices with dimensions:

$$
\left(N_{e}^{\mathrm{FO}}+N_{e}^{\mathrm{Bkgd}}+N_{\mu}\right) \times\left(N_{e}^{\mathrm{FO}}+N_{e}^{\mathrm{Bkgd}}+N_{\mu}\right)
$$


where FO refers to the fully oscillated events introduced in Eq.(4.3) which are used to calculate the predicted oscillation signal events, and we distinguish them from the background ( $B G$ events). Note that $N_{e}^{\mathrm{FO}}=N_{e}^{\mathrm{Bkgd}}=N_{e}$. A schematic representation of this extended error matrix is shown in Figure 4.2.

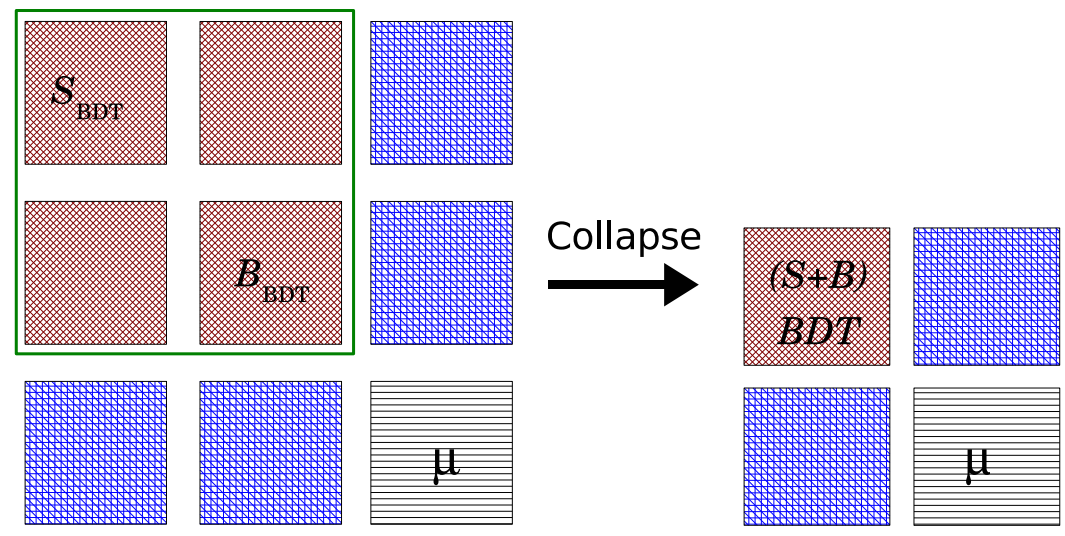

Expanded matrix

Figure 4.2: Illustration of the extended error matrix used in the BDT analysis. The matrix on the left is composed of a $\nu_{e}$ signal block (S), a $\nu_{e}$ background block (B), and a $\nu_{\mu} \mathrm{CCQE}$ block $(\mu)$, and their correlations. The matrix on the right has combined the $S$ and $B$ components and their correlations with the $\mu$ component.

In Figure 4.2 we illustrate the procedure to obtain from such an extended matrix, the error matrix with the signal and background $(S+B)$ contributions combined. This is simply done by adding together the $S$ and $B$ blocks of the extended matrix (red blocks) and the blocks containing the $S / \mu$ and $B / \mu$ correlations (blue blocks). Note that the $S$ and $B$ blocks have the same dimension $N_{e} \times N_{e}$.

The extended matrix is constructed by forming the histogram of the reconstructed neutrino energy $\left(E_{\nu}^{Q E}\right)$ for both, the $\nu_{\mu}$-CCQE candidates $\left(N_{\mu}\right.$ bins $)$ and $\nu_{e}$ candidates ( $N_{e}$ bins), and calculate the covariance matrix for the contents of the combined $N_{e}+N_{\mu}$ bin histogram using the following procedure. 
Covariance of the $E_{\nu}^{Q E}$ bin contents:

For each uncertainty source, points in its underlying parameter space are drawn from an assumed multivariate Gaussian distribution. Each chosen point will induce a distortion of the distributions of any observable in both the $\nu_{\mu}$ and $\nu_{e}$ samples.

Consider the distribution of a variable $X$ in $N_{b}$ bins. Let $x_{i}$ be the bin contents of bin $i$ of the distribution. For the purposes of this analysis, $X=E_{\nu}^{Q E}(-\mathrm{BDT}$, or -TBL.

Suppose there are $N_{\text {sys }}$ sources of systematic error, each associated with a parameter $p_{j}, j=1, \ldots, N_{\text {sys }}$, and suppose that the $N_{\text {sys }} \times N_{\text {sys }}$ covariance matrix $\mathcal{M}_{p}$ of the systematic error parameters is known. We use this matrix to sample the $N_{\text {sys }}{ }^{-}$ dimensional space of the parameters $p_{j}$ from a multivariate distribution assumed to be Gaussian. Each point $\mathbf{p}=\left(p_{1}, \ldots, p_{N_{s y s}}\right)$ sampled from this space according to the covariance matrix, will produce a slightly different distribution of variable $X$ from the one obtained from any other point. Suppose we sample $N_{\text {multi }}$ of such points.

We calculate the covariance between the number of events in each bin of the distribution of $X$ with respect to the distribution defined by the default values of the parameters as follows:

$$
\left\langle\sigma_{i} \sigma_{j}\right\rangle=\frac{1}{N_{\text {multi }}-1} \sum_{\alpha=1}^{N_{\text {multi }}}\left(x_{i}^{0}-x_{i}^{\alpha}\right)\left(x_{j}^{0}-x_{j}^{\alpha}\right)
$$

where $x_{i}^{0}$ is the value of the bin contents when the systematic parameters take their mean values $p_{j}^{0}, j=1, \ldots, N_{\text {sys }}$, and define the Central Value (CV) MC simulation. The values $x_{i}^{\alpha}, \alpha=1, \ldots, N_{\text {multi }}$, represent the distributions obtained for multisim $\alpha$ after varying the systematic parameters away from their means $p_{j}^{0}, j=1, \ldots, N_{\text {sys }}$ :

$$
\text { multisim } \alpha: \mathbf{p}^{\alpha}=\left(p_{1}^{0}+\Delta p_{1}^{\alpha}, \ldots, p_{N_{s y s}}^{0}+\Delta p_{N_{s y s}}^{\alpha}\right)
$$


In the case of uncertainties estimated from single excursions or unisims as defined earlier, the covariance matrix in bins of variable $X$ is calculated from the first derivative matrix $F_{i l}$ defined as:

$$
F_{i l} \equiv\left(\frac{\Delta x_{j}}{\Delta p_{l}}\right) \sigma_{p_{l}} \quad i=1, \ldots, N_{b} \quad l=1, \ldots, N_{s y s}
$$

where $N_{\text {uni }}$ is the number of uncertainty sources estimated in this way $\left(N_{\text {uni }}=2\right.$ in the present analysis). It is assumed that the change in $\Delta x_{i}$ caused by the unisim excursion corresponds to a $1 \sigma$ variation, and therefore $\sigma_{p_{l}} / \Delta p_{l} \approx 1$, leaving $F_{i l} \approx \Delta x_{i}$. The covariance in bins of the quantity $X$ is then given by

$$
\left\langle\sigma_{i} \sigma_{j}\right\rangle=\sum_{l, m}^{N_{\mathrm{uni}}} F_{i l} \times \delta_{l m} \times F_{j m}
$$

where the sources of uncertainty associated with the unisim excursions have been assumed to be uncorrelated. The expression in Eq.(4.9) assumes that the distribution of variable $X$ is a linear function of the systematic parameters that define the unisim excursion, and that the parameters are all linearly correlated.

If one uses a multisim approach to calculate the covariance between the contents of the bins of $X$, then one assumes that the correlations between the parameters $p_{j}$ are linear, while taking care of the nonlinear dependence of the distribution of $X$ on the parameters $p_{j}$. For details on this point see Ref.[166].

The covariance matrices constructed in this way are then expressed as fractional error matrices by dividing $\left\langle\sigma_{i} \sigma_{j}\right\rangle$ by the contents in the $\mathrm{CV}$ simulation that was used to produce them $\left(x_{i}^{0}\right)$. In this way, We obtain a collection of fractional error matrices $M_{i j}^{\prime}$ which can be weighted to the appropriate number of signal and background events by multiplying the fractional matrices by the predicted number of signal and background events in each bin $t_{i}^{\text {sig }}, t_{i}^{\text {bgd }}$ :

$$
M_{i j}^{S}=t_{i}^{\mathrm{sig}, \mathrm{bgd}} \times M^{S^{\prime}} \times t_{j}^{\mathrm{sig}, \mathrm{bgd}}
$$


where $S$ is any of the 9 sources of systematic error, $M^{S^{\prime}}{ }_{i j}$ is the fractional error matrix for source $S$, and $t_{i}^{\mathrm{sig}}\left(t_{i}^{\mathrm{bgd}}\right)$ is the predicted number of signal (background) events that contribute to the total predicted number of events $t_{i}$ in Eq.(4.2). This method allows to calculate the error matrix for any value of the oscillation parameters and scaling factors $f_{\pi}$ and $f_{K}$.

The contribution of the various systematic uncertainty sources as extracted from this method are shown in Table 4.2 and Table 4.3 for the $\nu_{e}$ signal and background contributions in the BDT analysis, in Table 4.5 and Table 4.6 for the $\nu_{e}$ signal and background contributions in the TBL analysis, and in Table 4.4, for the $\nu_{\mu}$ CCQE sample. The uncertainties on these tables are expressed in percent of the total number of events in the energy range of $300-1600 \mathrm{MeV}$ for the BDT analysis and from 475$3000 \mathrm{MeV}$ for the TBL analysis. The error estimates in these tables include all the correlations between the different contributions to the total error.

For example, the total error of $30.6 \%$ shown in the last column of Table 4.2 for the fully oscillated $\nu_{\mu} \rightarrow \nu_{e}$ events passing the BDT cuts is made up predominantly from the $32.1 \%$ uncertainty affecting the CCQE events (90.2\% of the total), and the $44.4 \%$ uncertainty affecting the $\mathrm{CC} 1 \pi$ events $(8.1 \%$ of the total). A significant positive correlation $\rho_{\mathrm{QE}, 1 \pi} \approx 0.41$ between these two contributions ${ }^{5}$ makes the total error smaller than the two independent contributions:

$$
\sigma_{\mathrm{Tot}}^{2} \approx\left(\sigma_{Q E}\right)^{2}+2 \times\left(\rho_{\mathrm{QE}, 1 \pi} \times \sigma_{Q E} \times \sigma_{1 \pi}\right)+\left(\sigma_{1 \pi}\right)^{2}=(30.6 \%)^{2}
$$

The other tables show similar effects coming from the correlations of the tabulated components.

\footnotetext{
${ }^{5}$ The numbers $\rho_{Q E, 1 \pi} \approx 0.41, \sigma_{Q E}=0.902 \times 32.1 \%=28.95 \%$, and $\sigma_{1 \pi}=0.081 \times 44.4 \%=3.6 \%$, in Eq.(4.11) can be determined directly from the information in Table 4.2.
} 
Table 4.2: Fractional systematic uncertainties (in percent) on the number of $100 \%$ full $\nu_{\mu} \rightarrow \nu_{e}$ transmutation events in the reconstructed neutrino energy range of $300 \mathrm{MeV}<$ $E_{\nu}^{Q E}$-BDT $<1600 \mathrm{MeV}$. The top row lists the two main types of interactions composing the sample (CCQE and $\mathrm{CC} 1 \pi$ ) with their contributions shown in the percentage in parenthesis. The various sources of systematic uncertainties are listed in the left column, and the total uncertainty associated with each source is listed in the right-most column. The bottom row shows the total systematic uncertainty of the component indicated in the top row.

\begin{tabular}{|r|c|c|c|}
\hline BDT 100\% $\left(\nu_{\mu} \rightarrow \nu_{e}\right)$ & $\nu_{e}\left(\bar{\nu}_{e}\right) \mathrm{CCQE}$ & $\nu_{e}\left(\bar{\nu}_{e}\right) \mathrm{CC} 1 \pi$ and other interactions & Total \\
& $(90.2 \%)$ & $(8.1 \%)$ & $100 \%$ \\
\hline$\pi^{+}$prod & 15.9 & 15.9 & 15.5 \\
$\pi^{-}$prod & 0.1 & 0.2 & 0.1 \\
$K^{+}$prod & - & - & 0.2 \\
$K_{L}^{0}$ prod & - & - & 0 \\
Beam Model & 3.9 & 6.5 & 4.1 \\
Cross Sections & 19 & 25.4 & 17.5 \\
NC $\pi^{0}$ yield & - & - & 0 \\
O.M. (multisims $)$ & 17.4 & 29.5 & 17.8 \\
O.M. (unisims $)$ & 10 & 12.9 & 7.5 \\
\hline Total & 32.1 & 44.4 & $\mathbf{3 0 . 6}$ \\
\hline \hline
\end{tabular}




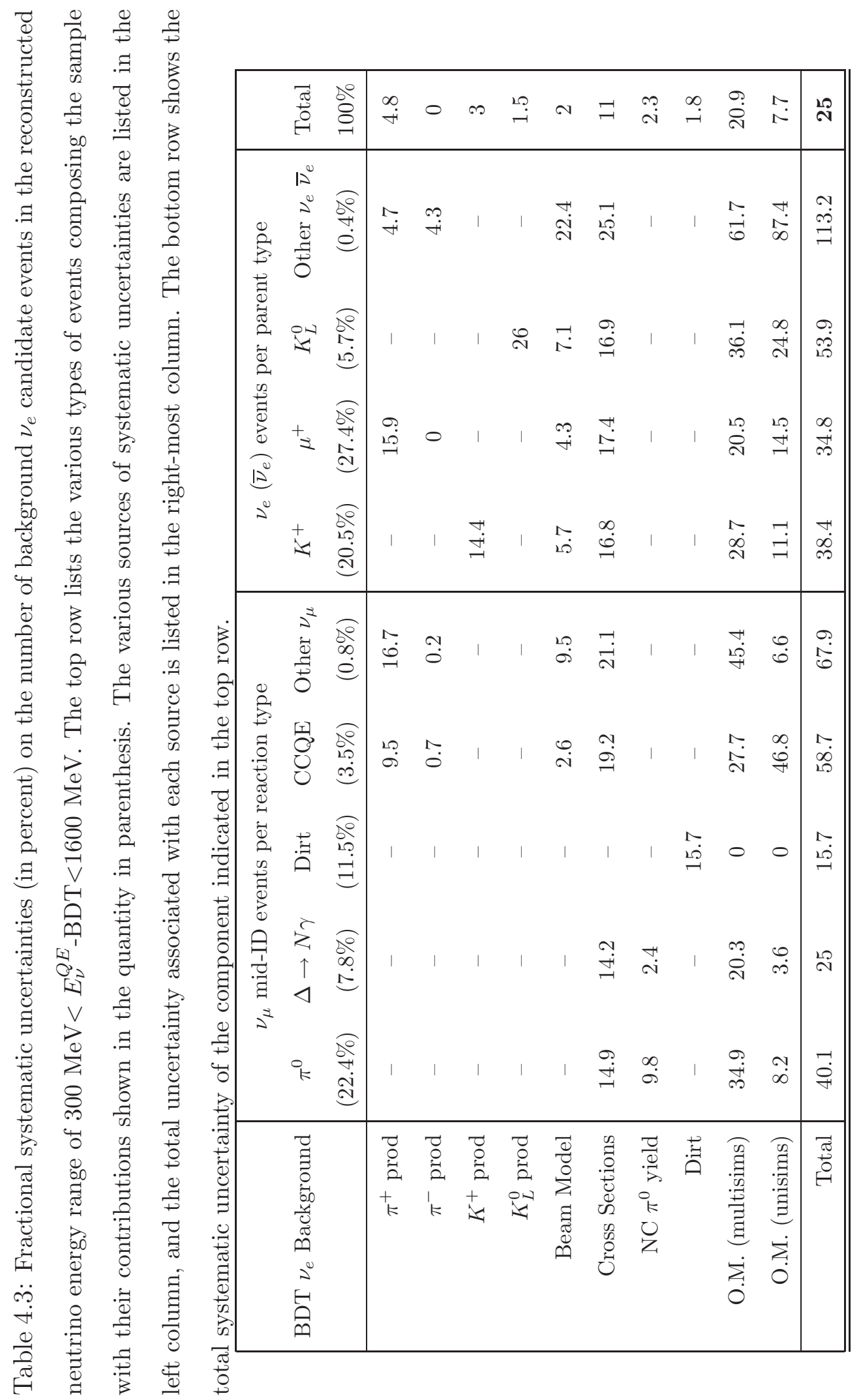




\subsection{3 $\quad \chi^{2}$ minimization procedure}

As mentioned in Section 4.2.2, depending on the region of the oscillation parameter space that is being sampled, a non-negligible number of signal events in the $\nu_{e}$ distribution can introduce additional contributions to the systematic error component of $M_{i j}$. We introduce the dependence of the error matrix on the oscillation parameters $\Delta m^{2}$ and $\sin ^{2} 2 \theta$ into our $\chi^{2}$ minimization with the iterative scheme described below.

\section{Grid search global scan}

The $\chi^{2}$ minimization is carried out over a grid of $190 \times 190$ points in the oscillation parameter space chosen to be uniform in logarithmic scale and to span the region of interest $^{6}$. The error matrix is first assumed to contain no signal contribution and is kept fixed while the predicted number of events $M C_{i}$ is varied over the grid to find the pair that gives the minimum $\chi_{1}^{2}$. The error matrix is then updated to contain the amount of signal found in the previous step and kept fixed again while the predicted number of events is varied in a second minimization that yields a value $\chi_{2}^{2}<\chi_{1}^{2}$. This iterative procedure is continued until the difference $\chi_{i}^{2}-\chi_{i-1}^{2}<10^{-3}$. Convergence is typically achieved after three iterations. Studies with fits to fake signals hove shown that typically the best fit point found in the first iteration moves by as much as one grid point in the second iteration, remaining fixed after the third. For fake fits to zero signal distributions one iteration of the error matrix proved enough. Failure to converge after a few iterations $(<6)$ was observed only for fake input signals exceeding $\sin ^{2} 2 \theta \gtrsim 10^{-2}$ and $\Delta m^{2} \gtrsim 10$.

\footnotetext{
${ }^{6}$ The interval $4 \times 10^{-4}<\sin ^{2} 2 \theta<1$, and $0.01 \mathrm{eV}^{2}<\Delta m^{2}<100 \mathrm{eV}^{2}$ was used for all calculations in this thesis.
} 
Table 4.4: Fractional systematic uncertainties (in percent) on the number of $\nu_{\mu}$ CCQE events in the reconstructed neutrino energy range of $0 \mathrm{MeV}<E_{\nu}^{Q E}-\mathrm{BDT}<1900 \mathrm{MeV}$. The top row lists the two main types of interactions composing the sample (CCQE and $\mathrm{CC} 1 \pi$ ) with their contributions shown in the percentage in parenthesis. The various sources of systematic uncertainties are listed in the left column, and the total uncertainty associated with each source is listed in the right-most column. The bottom row shows the total systematic uncertainty of the component indicated in the top row.

\begin{tabular}{|r|cc|c|}
\hline$\nu_{\mu}$ CCQE candidates & $\begin{array}{c}\nu_{\mu} \mathrm{CCQE} \\
(72.6 \%)\end{array}$ & $\nu_{\mu} \mathrm{CC} 1 \pi$ and other interactions & Total \\
& 16.2 & $(27.5 \%)$ & $100 \%$ \\
\hline$\pi^{+}$prod & 0.1 & 15.9 & 15.8 \\
$\pi^{-}$prod & - & 0.2 & 0.1 \\
$K^{+}$prod & - & - & 0.2 \\
$K_{L}^{0}$ prod & 3.7 & - & 0 \\
Beam Model & 19.3 & 5.3 & 4 \\
Cross Sections & - & 23.8 & 15.5 \\
NC $\pi^{0}$ yield & - & - & 0 \\
Dirt & 1.9 & - & 0 \\
O.M. (multisims) & 2.9 & 9.4 & 3.4 \\
O.M. (unisims) & 25.7 & 3.9 & $\mathbf{2 3}$ \\
\hline Total & \multicolumn{2}{|}{} \\
\hline \hline
\end{tabular}

\section{The raster scan method}

In this Section we describe the procedure that was used to construct the 90\% C.L. limits to oscillations for the BDT analysis in [190] and throughout the rest of this work. The raster scan method is a common way to display the result of null experiments and was chosen in order to compare with older results, and it attempts to answer the specific question of what range of $\sin ^{2} 2 \theta$ values are allowed for a given assumption of the true value of $\Delta m^{2}$. 
For each $\Delta m_{i}^{2}$ value, $i=1, \ldots, N_{\Delta m^{2}}$ :

1. Loop over the $\sin ^{2} 2 \theta$ values to find the $\sin ^{2} 2 \theta_{i}^{\text {bf }}$ that minimizes $\chi^{2}$ using an error matrix with no signal.

2. Update the error matrix to contain the found value $\sin ^{2} 2 \theta_{i}^{\text {bf }}$ and the current $\Delta m_{i}^{2}$ value. This defines a matrix $M_{i}^{\mathrm{bf}}$.

3. Loop over the $\sin ^{2} 2 \theta$ values again and calculate $\Delta \chi_{i}^{2}$ for the current $\Delta m_{i}^{2}$ value as follows:

$$
\Delta \chi_{i}^{2}(x)=\chi^{2}\left(M_{i}^{\mathrm{bf}}, x\right)-\chi^{2}\left(M_{i}^{\mathrm{bf}}, \sin ^{2} 2 \theta_{i}^{\mathrm{bf}}\right)
$$

where the first argument of $\chi 2$ is the matrix used and the second is the $\sin ^{2} 2 \theta$ value. Both use the current $\Delta m_{i}^{2}$ to determine the amount of signal present.

The set of curves $\Delta \chi_{i}^{2}(x)$ for all $i$, are functions of $\sin ^{2} 2 \theta$ and define a surface that is then used to determine confidence level limits by moving away from $\sin ^{2} 2 \theta_{i}^{\text {bf }}$ to reach a specified $\Delta \chi_{i}^{2}$ (e.g. $\Delta \chi_{i}^{2}=1.64$ for one-sided $90 \%$ C.L. limits).

Several thousand fits to fake experiments with zero and non zero input oscillation signals have been performed to test the robustness of the minimization procedures. The results have the expected behavior giving $\Delta \chi^{2}$ distributions with $\sim 2$ dof in most of the parameter space. Failure to converge in less than 2 iterations occurred only for input signals around $\Delta m^{2}>10 \mathrm{eV}^{2}$ and $\sin ^{2} 2 \theta>0.01$, but behaving well in all other cases. Examples of fits to fake data sets can be seen in Appendix F. A frequentist calculation using the 2D fitting technique was performed and it was found that the limits obtained by requiring a $\Delta \chi^{2}$ change of 4.61 (called global-scan for 2 dof Gaussian assumption) are close to the frequentist result and disagree slightly only in the region of $\Delta m^{2}>1 \mathrm{eV}^{2}$. This study is described in Appendix C. 


\subsection{4 $\nu_{\mu}$-constrained number of $\nu_{e}$ 's}

Besides constraining the magnitude of the uncertainties in the number of $\nu_{e}$ events, the observed $\nu_{\mu}$ energy distribution and the correlations between the two samples provide a means to correct the shape and normalization of the predicted $\nu_{e}$ distribution.

Although no correction ${ }^{7}$ is applied to the $\nu_{e}$ distribution, the presence of the offdiagonal terms in the $\chi^{2}$ Eq.(4.1) Will allow solutions in which the $\nu_{e}$ and $\nu_{\mu}$ bins display similar discrepancies with respect to the data ${ }^{8}$ while giving an acceptable $\chi^{2}$ value. As a result an excess of events in the $\nu_{e}$ distribution will only be ascribed to an oscillation signal if it is larger than the differences that can be accounted for through the $\nu_{\mu} / \nu_{e}$ correlations. An analytical example showing how this occurs in the case of $1 \nu_{e}$ bin and $1 \nu_{\mu}$ bin is given in Appendix B.

To explicitly calculate what the equivalent correction is, one can attempt to solve for the number of events in the $\nu_{e}$ and $\nu_{\mu}$ energy distributions that minimize the following $\chi^{2}$ function:

$$
\begin{gathered}
\chi^{2}=\sum_{i j}^{n_{\nu_{e}}+n_{\nu_{\mu}}} \Delta_{i} M_{i j}^{-1} \Delta_{j}+\sum_{k}^{n_{\nu_{\mu}}} \frac{\left(N_{k}^{f i t}-N_{k}^{\text {data }}\right)^{2}}{N_{k}^{\text {data }}} \\
\frac{\partial \chi^{2}}{\partial N_{i}^{f i t}}=0
\end{gathered}
$$

where $\Delta_{i}=N_{i}^{f i t}-N_{i}^{M C}$ is the difference between the fitted number of events and the Monte Carlo prediction. The set of equations that need to be solved is:

$$
\frac{\partial \chi^{2}}{\partial N_{i}^{f i t}}=2 \sum_{j=1}^{n_{\nu_{e}}+n_{\nu_{\mu}}} M_{i j}^{-1} \Delta_{j}+2 \frac{\left(N_{i}^{f i t}-N_{i}^{\text {data }}\right) \delta_{i\left(\nu_{\mu} \text { bin }\right)}}{N_{i}^{\text {data }}}=0
$$

where $\delta_{i\left(\nu_{\mu} \text { bin }\right)}$ is equal to 1 when $i$ is a $\nu_{\mu}$ bin, and zero otherwise. Defining the matrix

\footnotetext{
7 Other than the $f_{\pi}=1.22$ scaling in the predicted number of events

8 For example, an overall residual normalization difference larger than the error bars.
} 
$B_{i j}^{-1}$ as follows:

$$
B_{i j}^{-1}=\left\{\begin{array}{l}
M_{i j}^{-1} \text { for } i \leq n_{\nu_{e}} \text { or } j \leq n_{\nu_{e}} \\
M_{i j}^{-1}+\frac{1}{N_{i}^{\text {data }}} \text { for } i>n_{\nu_{e}} \text { and } j>n_{\nu_{e}}
\end{array}\right.
$$

we can write Eq.(4.13) in the following form:

$$
\sum_{i j} B_{i j}^{-1} N_{j}^{f i t}=\sum_{j} M_{i j}^{-1} N_{j}^{M C}+\frac{N_{i}^{\text {data }} \delta_{i\left(\nu_{\mu} \mathrm{bin}\right)}}{N_{i}^{\text {data }}}
$$

The solution of this set of equations is

$$
N_{i}^{f i t}=\sum_{k} B_{i k}\left(\sum_{j} M_{k j}^{-1} N_{j}^{M C}+\delta_{k\left(\nu_{\mu} \mathrm{bin}\right)}\right)
$$

and the covariance matrix for the $N_{i}^{f i t}$ values is given by

$$
\left\langle\delta N_{i}^{f i t} \delta N_{j}^{f i t}\right\rangle=B_{i j}
$$

The errors contained in the matrix $B_{i j}$ have been effectively constrained by the high number of events in the $\nu_{\mu}$ bins.

The transformations in Eq.(4.14) and Eq.(4.16) are used throughout this thesis only to display the results of the fitted distributions and constrained error bands, and are not part of the fitting machinery.

\subsection{Oscillations results with the BDT analysis}

In Section 4.1 we showed the comparison of the energy distributions of the BDT $\nu_{e}$ candidate events in data and Monte Carlo before the fit is performed (middle plot in Figure 4.1). The uncertainties shown there are those obtained directly from the multisim procedure, and correspond to a suitable combination of the numbers in Table 4.2 and Table 4.3 for the $\nu_{e}$ sample, and Table 4.4 for the $\nu_{\mu}$ sample. 

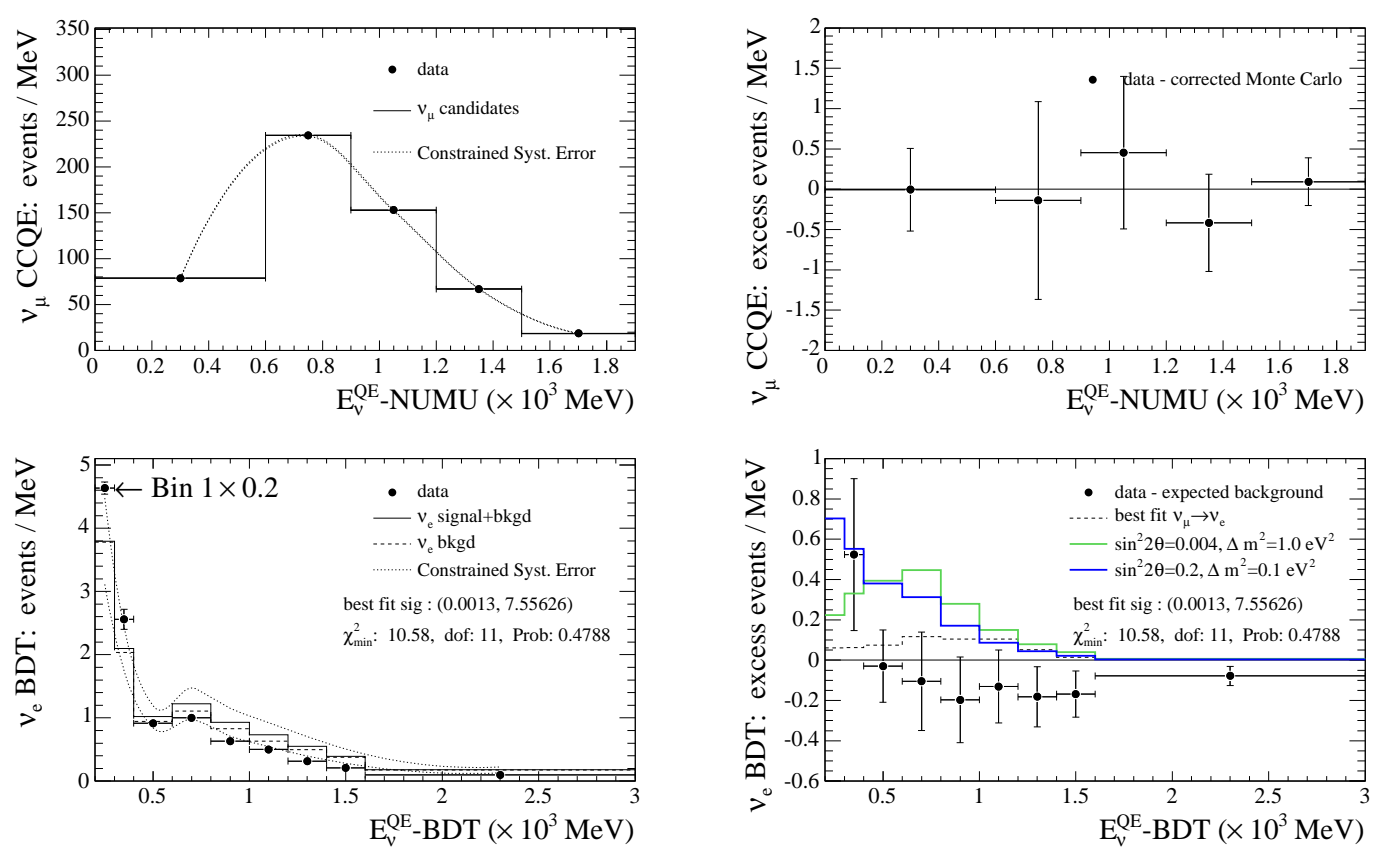

(a) After Fit, Constrained errors.

(b) After Fit, background subtraction.

Figure 4.3: The reconstructed neutrino energy distributions $\left(E_{\nu}^{Q E}-\mathrm{BDT}\right)$ for $\nu_{\mu} \mathrm{CCQE}$ and $\nu_{e}$ candidate samples in the BDT analysis after constraints and corrections. The best fit parameters $\left(\sin ^{2} 2 \theta, \Delta m^{2}\right)$ and goodness of fit values are shown in the figures. In the background subtracted distributions in (b) the points have total errors. 


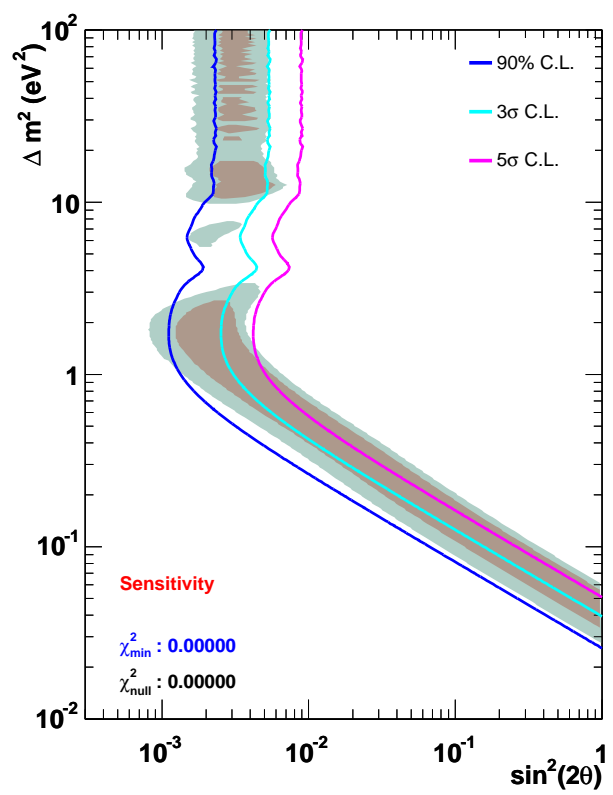

(a) BDT sensitivity to oscillations.

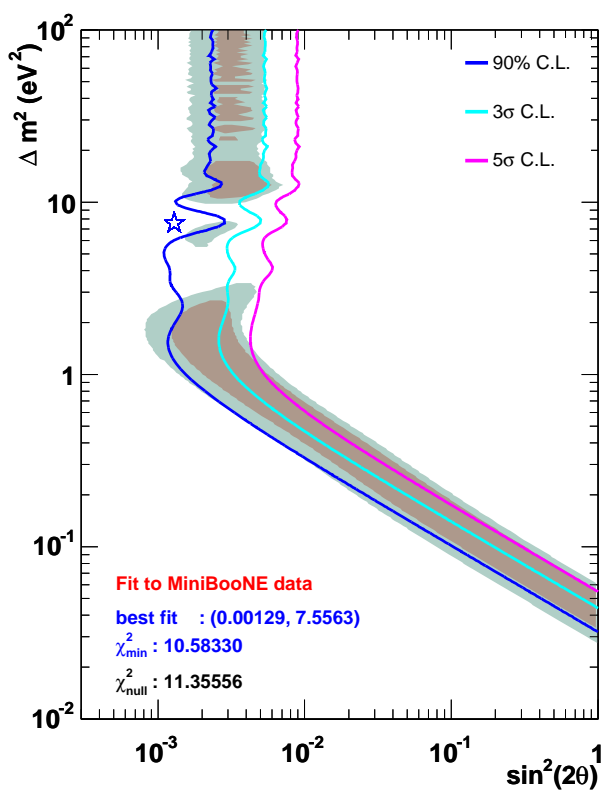

(b) BDT analysis limit on open data.

Figure 4.4: The 90\% (blue), $3 \sigma$ (cyan), and $5 \sigma$ (magenta) sensitivity curves and limits to oscillations of the BDT analysis. The 90\% C.L. limit shown in (b) reproduces the one appearing in [190]. 
Figure 4.3 compares the energy distributions after the fit is performed on the open data in the full energy range from $300 \mathrm{MeV}$ to $3000 \mathrm{MeV}$. The fit yields a $\chi^{2}$ probability of $41 \%$ for the null hypothesis $\left(\chi_{n u l l}^{2} / n d f=11.36 / 11\right)$, and $48 \%$ for the best fit point $\left(\chi_{b f}^{2} / n d f=10.58 / 11\right)$ at $\Delta m^{2}=5.56 \mathrm{eV}^{2}$, and $\sin ^{2} 2 \theta=0.0013$. We stress that the number of degrees of freedom was reduced by one by virtue of the scaling by a factor of 1.22 applied to correct the $\nu_{\mu}$ CCQE normalization as described at the end of Section 3.5.3. The top plot in Figure 4.3(a) shows the reconstructed neutrino energy distribution $E_{\nu}^{Q E}$-BDT of the $\nu_{\mu}$ CCQE events in data (points), and Monte Carlo after the correction of Eq.(4.16), which has become nearly identical to the data distribution. The fine dotted lines represent the constrained systematic error band, which has become of the size of the statistical error in the sample after applying the transformation $\mathrm{Eq}(4.14)$. The residual differences of data minus simulation are shown in the top plot of Figure 4.3(b).

The solid line in bottom plot in Figure 4.3(a) shows the predicted number of background $\nu_{e}$ candidate events after the correction of Eq.(4.16), and the dashed line is the background plus fitted signal. The dotted lines represent the constrained errors on the fitted total number of events in each bin as calculated with Eq.(4.14). The points are the distribution from the data, which agrees with the prediction within uncertainties. The background subtracted distribution of events in the bottom plot of Figure 4.3(b) shows that there is a net deficit of data events with respect of the expected number. the dashed line in the figure shows the result of the fit. For comparison, two LSND like signals are shown in the red and blue curves.

We stress that the Monte Carlo distributions in Figure 4.3 have been slightly modified from the original ones in Figure 4.1 by using the transformation in Eq.(4.16) to represent the constraining effect of the $\nu_{\mu}$ CCQE sample during the fitting procedure. Any remaining differences in the $\nu_{\mu} \mathrm{CCQE}$ distribution after the normalization scal- 
ing of 1.22 mentioned in Section 3.5.3 are further reduced with this procedure. In this transformation also the number of $\nu_{\mu}$ CCQE events is modified to be as close as possible to the data distribution, The details of the correction applied to the $\nu_{\mu}$ CCQE distribution depend also on the $\nu_{e}$ distribution and the total error matrix.

Figure 4.4(a) shows the expected $90 \%, 3 \sigma$, and $5 \sigma$ oscillation sensitivity curves that are expected for the BDT analysis using this technique. These limits are calculated by fitting a fake data sample identical to the background prediction (no signal) with the procedure described in Section 4.2.3. Figure 4.4(b) shows the 90\%, 3o, and $5 \sigma$ C.L. limits in the oscillation parameter space obtained from fitting the MiniBooNE open data set. The fit is consistent with no oscillations. The resulting exclusion region in the oscillation parameter space shown in Figure 4.4(b) reproduces the result in $[190]$. 
Table 4.5: Fractional systematic uncertainties (in percent) on the number of $100 \%$ full $\nu_{\mu} \rightarrow \nu_{e}$ transmutation events in the reconstructed neutrino energy range of $475 \mathrm{MeV}<$ $E_{\nu}^{Q E}-\mathrm{TBL}<1250 \mathrm{MeV}$. The top row lists the two main types of interactions composing the sample (CCQE and $\mathrm{CC} 1 \pi$ ) with their contributions shown in the percentage in parenthesis. The various sources of systematic uncertainties are listed in the left column, and the total uncertainty associated with each source is listed in the right-most column. The bottom row shows the total systematic uncertainty of the component indicated in the top row.

\begin{tabular}{|r|c|c|c|}
\hline TBL $100 \%\left(\nu_{\mu} \rightarrow \nu_{e}\right)$ & $\begin{array}{c}\nu_{e}\left(\bar{\nu}_{e}\right) \mathrm{CCQE} \\
(84.5 \%)\end{array}$ & $\begin{array}{c}\nu_{e}\left(\bar{\nu}_{e}\right) \mathrm{CC} 1 \pi \text { and other interactions } \\
(14.0 \%)\end{array}$ & $\begin{array}{c}\text { Total } \\
\pi^{+} \text {prod }\end{array}$ \\
$\pi^{-}$prod & 16 & 15.8 & 15.7 \\
$K^{+}$prod & -.1 & 0.3 & 0.1 \\
$K_{L}^{0}$ prod & - & - & 0.1 \\
Beam Model & 3.5 & - & 0 \\
Cross Sections & 19.3 & 4.8 & 3.6 \\
NC $\pi^{0}$ yield & - & 21.8 & 16.8 \\
O.M. (multisims) & 4.1 & - & 0 \\
O.M. (unisims) & 7.3 & 21 & 5 \\
\hline Total & 26.7 & 24.7 & 2.5 \\
\hline \hline
\end{tabular}




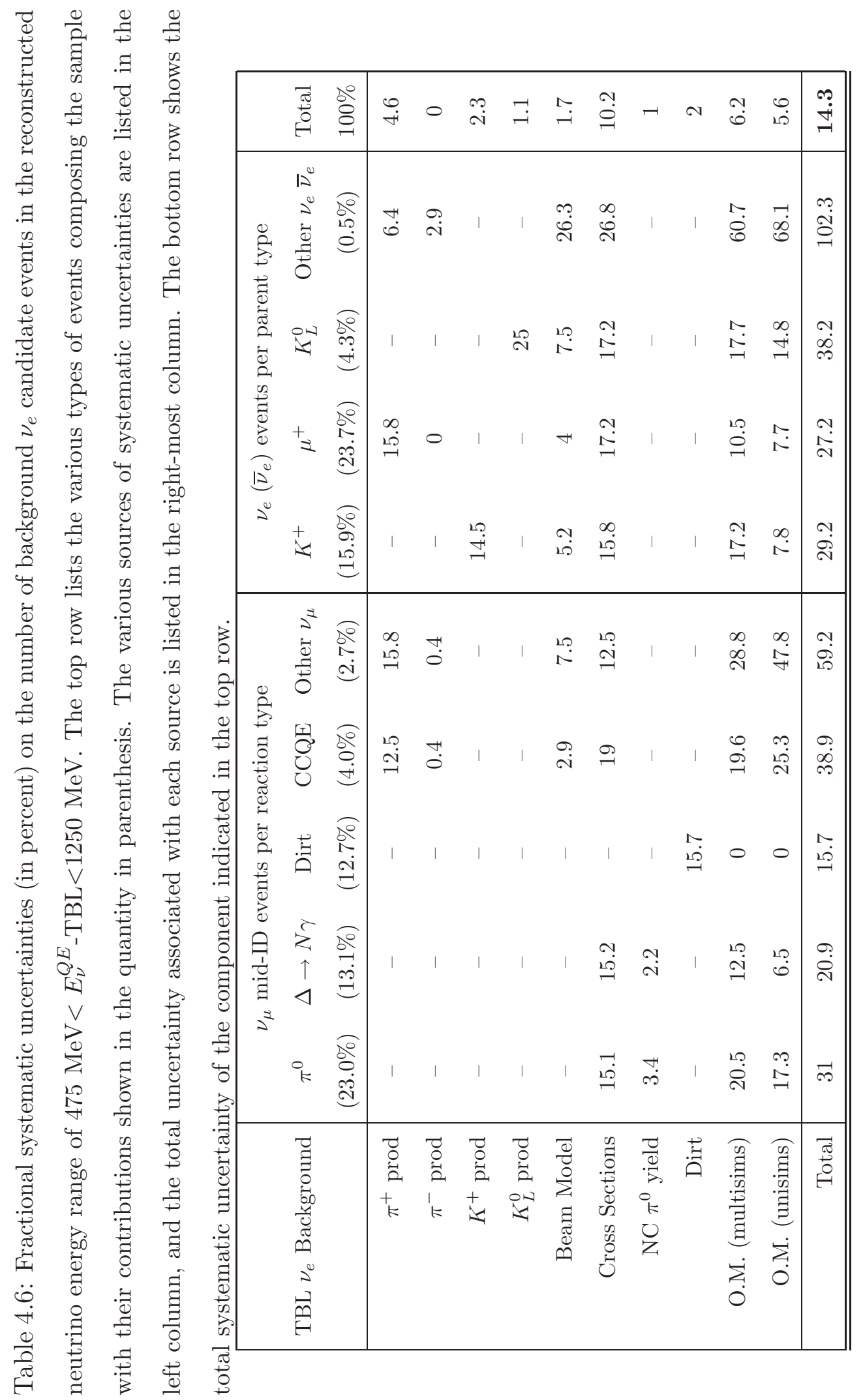




\subsection{Oscillations results with the TBL analysis}

The techniques used in the official TBL analysis are significantly different from those presented here, but they both aim to constrain the predicted $\nu_{e}$ distribution by using the high statistics of the $\nu_{\mu}$ CCQE sample and the correlations between them. As has been seen in the previous section, bringing the $\nu_{\mu}$ CCQE sample into the oscillations fit has the desired effect.

The TBL analysis in [190] performed corrections and constrained the systematic errors before making a fit for two neutrino oscillations. This technique, described elsewhere [180], ultimately uses the same information as the present fit technique, and therefore it is expected to yield comparable results.

In the present analysis we show that one can achieve a similar sensitivity to oscillations by applying the combined fit technique to the TBL $\nu_{e}$ candidate sample.

We use the TBL selection cuts and neutrino energy calculation to form the $\nu_{e}$ candidate sample, and construct the full covariance matrix of this distribution with the $\nu_{\mu}$ CCQE sample used for the BDT analysis. It is important to note that the $\nu_{\mu}$ CCQE sample used here is not the same as that used to constrain the $\nu_{e}$ prediction in the TBL analysis in [190], and therefore some differences are expected.

The systematic uncertainties for the TBL analysis $\nu_{e}$ candidate sample were calculated as described in Section 4.2.2 and are shown in Table 4.5 for the signal component and in Table 4.6 for the background events.

\subsubsection{Treatment of Optical Model uncertainties}

An important difference between the TBL and BDT analyses is the estimation of the detector optical model (O.M.) uncertainties. The estimation of this particular source 

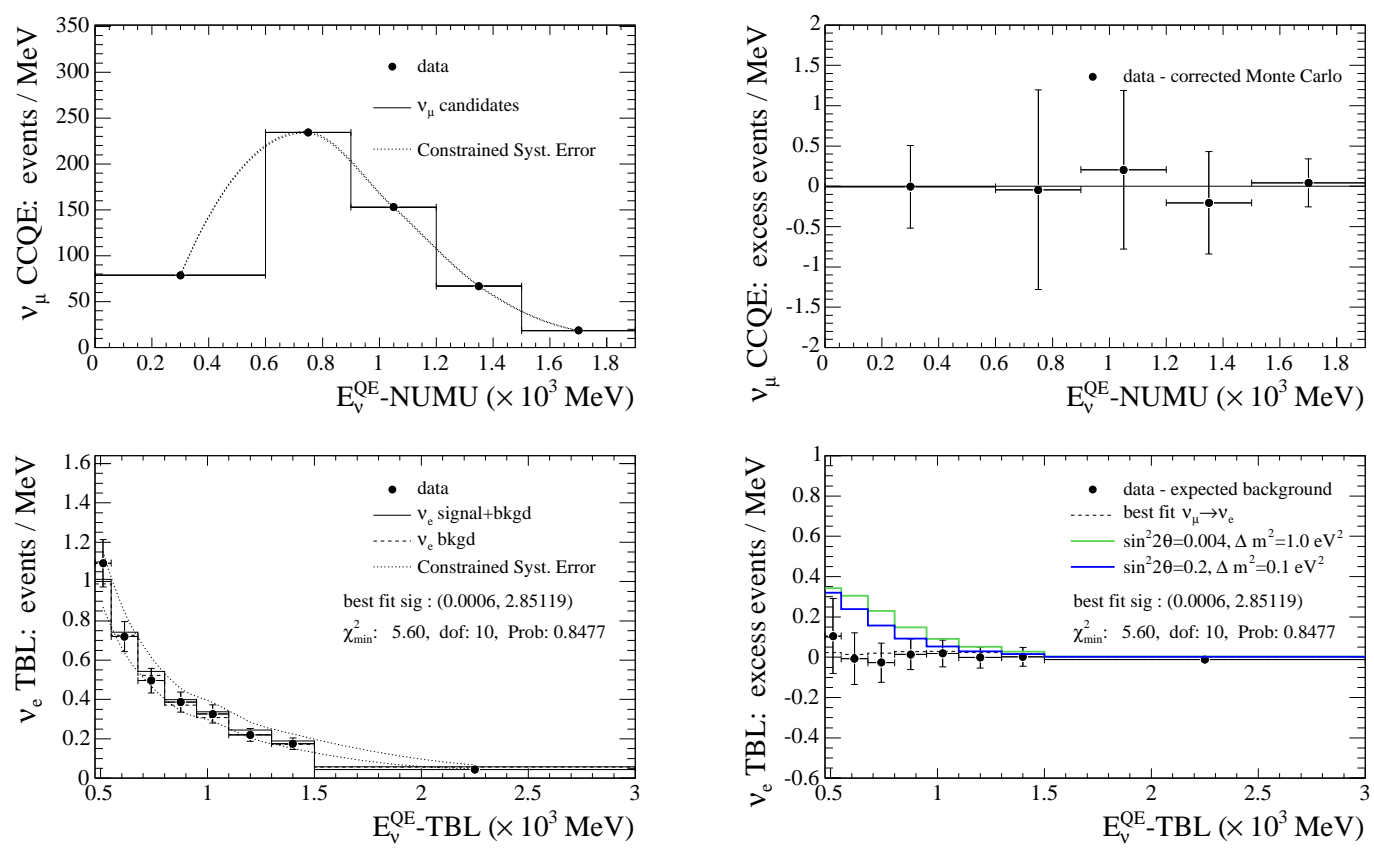

(a) After Fit, Constrained errors.

(b) After Fit, background subtraction.

Figure 4.5: Energy distributions for the TBL analysis result with the present technique. The best fit parameters $\left(\sin ^{2} 2 \theta, \Delta m^{2}\right)$ and goodness of fit values are shown in the figures. In the background subtracted distributions in (b) the points have total errors. 


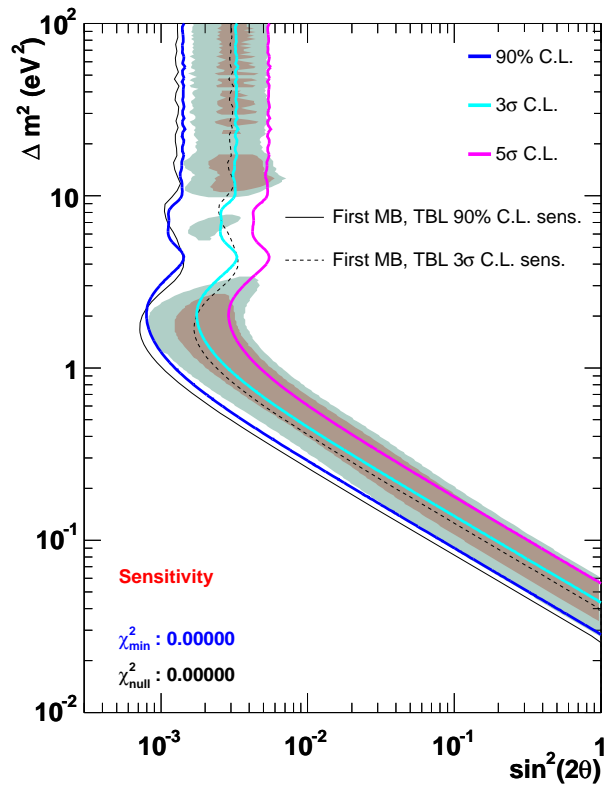

(a) TBL sensitivity to oscillations.

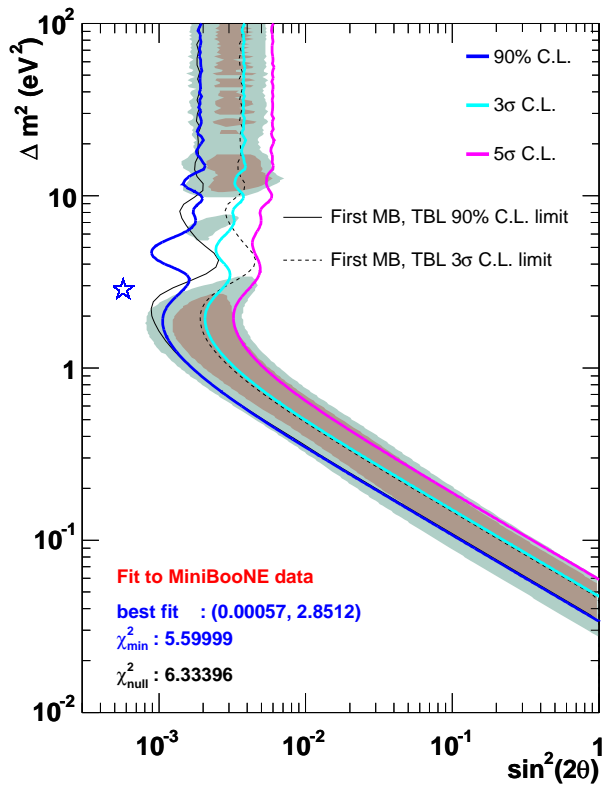

(b) TBL analysis limit on open data.

Figure 4.6: The $90 \%$ (blue), $3 \sigma$ (cyan), and $5 \sigma$ (magenta) sensitivity curves (a) and limits to oscillations (b) obtained with the present technique using the TBL selection cuts for the $\nu_{e}$ candidate sample. For comparison, the solid and dashed black lines show the result from the technique used in [190]. 
of error used 66 hit-level simulations of the experiment ${ }^{9}$ with varying optical model parameters drawn from the estimated covariance ellipsoid to construct a reliable error matrix. The method used to estimate the underlying covariance of the 35 optical model parameters was described in Section 3.3.1. Each hit-level simulation has the statistics of the data sample (corresponding to $5.7 \times 10^{20}$ P.O.T.) and therefore already contains the statistical uncertainty of the data.

The BDT analysis used an alternative procedure to remove the contribution of statistical uncertainty from the O.M. variations. A $5^{\text {th }}$ degree polynomial was fit to the distribution $x_{i}^{0}-x_{i}^{\alpha}, i=1, \ldots, N_{b}$ in Eq.(4.7) before calculating the covariance matrix. This smoothing procedure can potentially incorrectly transform statistical fluctuations into part of the systematic error that is being estimated, and therefore represents an upper bound to the desired uncertainty.

It was observed that for the BDT analysis the unsmoothed O.M. error matrix induced a pathological behavior in the optimization code used to determine the BDT score cuts as a function of $E_{\nu}^{Q E}$-BDT, and on this basis it was decided to use the slightly overestimated O.M. uncertainties obtained with the smoothing procedure, with which the optimization code behaved properly.

It is clear from this discussion that in order to compare the results from the present analysis using the TBL selection cuts with the results in [190] we need to use the unsmoothed O.M. error matrix.

The results using the unsmoothed OM matrices and the TBL selection cuts with the present fitting technique are shown in Figure 4.5, and the corresponding sensitivity and limit to oscillations are shown in Figure 4.6.

The fit over the $475 \mathrm{MeV}<E_{\nu}^{Q E}-\mathrm{TBL}<3000 \mathrm{MeV}$ energy range yields a $\chi^{2}$ probability of $79 \%$ for the null hypothesis $\left(\chi_{\text {null }}^{2} / n d f=6.33 / 10\right)$, and $85 \%$ for the best fit

\footnotetext{
${ }^{9}$ In the notation of Section 4.2.2 $N_{\text {multi }}=66$ for the O.M. errors
} 
point $\left(\chi_{b f}^{2} / n d f=5.60 / 10\right)$ at $\Delta m^{2}=2.58 \mathrm{eV}^{2}$, and $\sin ^{2} 2 \theta=0.0006$. The number of degrees of freedom is again reduced by one to account for the scaling factor of 1.22 applied to correct the $\nu_{\mu}$ CCQE normalization as explained near the end of Section 3.5.3.

Figure 4.5(a) shows the reconstructed energy distributions of the $\nu_{\mu}$ CCQE sample $\left(E_{\nu}^{Q E}-\mathrm{BDT}\right)$ and the TBL $\nu_{e}$ candidate sample $\left(E_{\nu}^{Q E}-\mathrm{TBL}\right)$ after the fit. The best fit oscillation parameters $\left(\sin ^{2} 2 \theta, \Delta m^{2}\right)=0.0006,2.851$, give a goodness of the fit $\chi^{2} / d o f=5.60 / 10$.

The top plot in Figure 4.5(b) shows the difference in data minus Monte Carlo for the $\nu_{\mu} \mathrm{CCQE}$ distributions, and the bottom plot the background-subtracted $\nu_{e}$ candidate distribution after the fit. The backgrounds have been corrected correction of Eq.(4.16), and the error bars have been constrained using Eq.(4.14).

Note that although we used the same $\nu_{\mu}$ CCQE sample, the top plot of Figure 4.5(b) is slightly different from the top plot in Figure 4.3(b). This is because the transformation Eq.(4.16) depends on the specific $\nu_{e}$ candidate distribution that is used, giving slightly different corrections to the Monte Carlo prediction of $\nu_{\mu} \mathrm{CCQE}$ events in the BDT and TBL cases.

It can be seen that the oscillations results have comparable statistical strength to those of Ref.[190], indicating that the two techniques used similar underlying information to constrain the $\nu_{e}$ sample. The differences in the detailed structure above $\Delta m^{2} \gtrsim 1 \mathrm{eV}^{2}$ are expected from our usage of a different $\nu_{\mu}$ CCQE sample in the construction of the $\nu_{\mu}-\nu_{e}$ correlations in the systematic error estimates. Although the same data and simulation were used for the $\nu_{e}$ candidate sample, we stress that the result in Ref.[190] used a different $\nu_{\mu}$ data set to determine the corrections to the $\nu_{e}$ prediction and to reduce the systematic uncertainties, and therefore, the detail in which the $\chi^{2}$ minimization responds to the fluctuations of the data in the 
determination of the C.L. contours is expected to be slightly different.

\subsubsection{TBL analysis with smoothed O.M. errors}

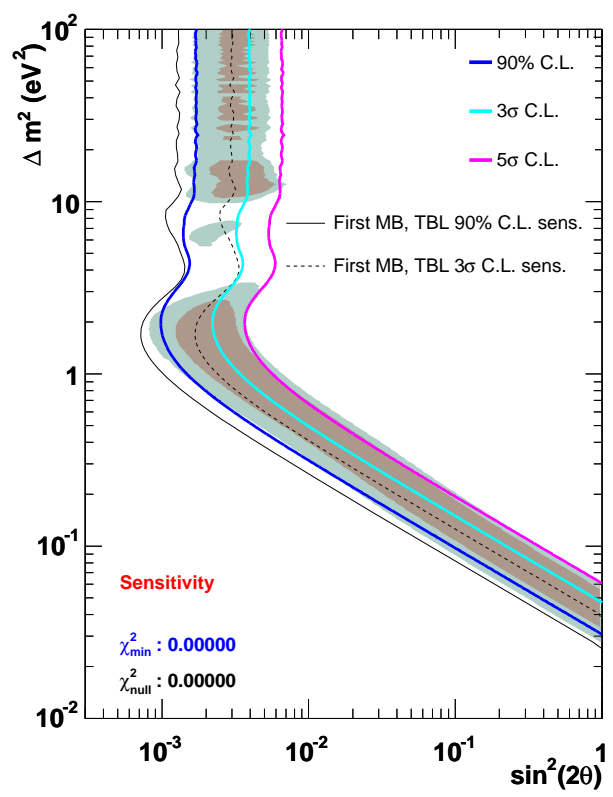

(a) TBL sensitivity to oscillations.

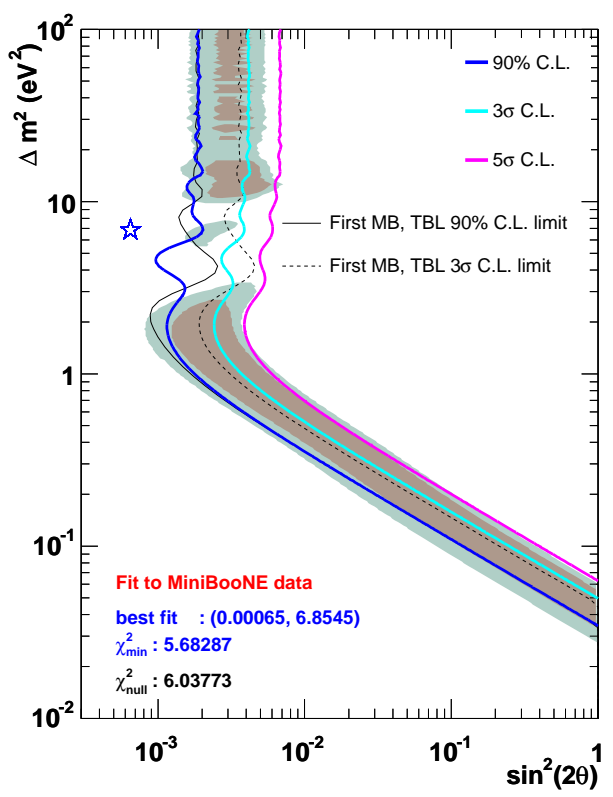

(b) TBL analysis limit on open data.

Figure 4.7: Results with the TBL selection using the smoothed O.M. errors I. The sensitivity (a) and limit (b) are slightly degraded with respect to the case of the unsmoothed O.M. errors (see text).

One can apply the combined fit technique to the TBL $\nu_{e}$ candidate sample using the O.M. errors estimated with the smoothing procedure described earlier. The result of this calculation is shown in Figure 4.7, showing that the sensitivity to oscillations becomes degraded, as does the result of fitting the open data, although the latter does so to a lesser extent.

The fit over the $475 \mathrm{MeV}<E_{\nu}^{Q E}-\mathrm{TBL}<3000 \mathrm{MeV}$ energy range yields a $\chi^{2}$ probability of $81 \%$ for the null hypothesis $\left(\chi_{\text {null }}^{2} / n d f=6.03 / 10\right)$, and $84 \%$ for the best fit point $\left(\chi_{b f}^{2} / n d f=5.68 / 10\right)$ at $\Delta m^{2}=0.010 \mathrm{eV}^{2}$, and $\sin ^{2} 2 \theta=0.0003$. 


\subsection{Combining the $\nu_{e} \mathrm{BDT}, \nu_{e} \mathrm{TBA}$, and $\nu_{\mu} \mathrm{CCQE}$ samples}

The method chosen to perform an oscillation analysis with both $\nu_{e}$ candidate samples was to extended the definition of the $\chi^{2}$ in Eq.(4.1) to include the bins of the BDT and TBL $\nu_{e}$ candidate samples together with the already used $\nu_{\mu}$ bins:

$$
\begin{aligned}
\chi^{2}\left(\Delta m^{2}, \sin ^{2} 2 \theta\right)=\sum_{i, j}^{N_{e}+N_{\mu}}\left(m_{i}-t_{i}\right) M_{i j}^{-1}\left(m_{j}-t_{j}\right) \\
\nu_{e} \text { BDT candidate bins: } i=1, \ldots, N_{e}^{\mathrm{BDT}} \\
\nu_{e} \text { TBL candidate bins: } i=N_{e}^{\mathrm{BDT}}+1, \ldots, N_{e}^{\mathrm{BDT}}+N_{e}^{\mathrm{TBL}} \\
\nu_{\mu} \text { candidate bins: } i=N_{e}^{\mathrm{BDT}}+N_{e}^{\mathrm{TBL}}+1, \ldots, N_{e}^{\mathrm{BDT}}+N_{e}^{\mathrm{TBL}}+N_{\mu}
\end{aligned}
$$

with similar nomenclature as in Eq.(4.1). From this expression we should expect an increase in the power of constraining the systematic uncertainties coming from the newly introduced correlations in the matrix $M_{i j}$, which include: (a) the correlations between the BDT and TBL signal events, (b) the correlations between the BDT and TBL background events, (c) correlations between the BDT signal (background) events and TBL background (signal) events, (d) the correlations of all of the $\nu_{\mu} C C Q E$ events with the BDT signal, BDT backgrounds, and with the TBL signal and background events.

This leads to a matrix with $5 \times 5$ blocks (as opposed to the $3 \times 3$ block structure used so far), which is illustrated in Figure 4.8. As was done before, this $5 \times 5$ matrix is formed by adding the contributions of all uncertainty sources, taking into account the contribution from a possible oscillation signal. The color of the blocks in the arrays shown in Figure 4.8 indicates the portions that need to be added together to combine the corresponding signal and background components on the left into a collapsed matrix on the right. 


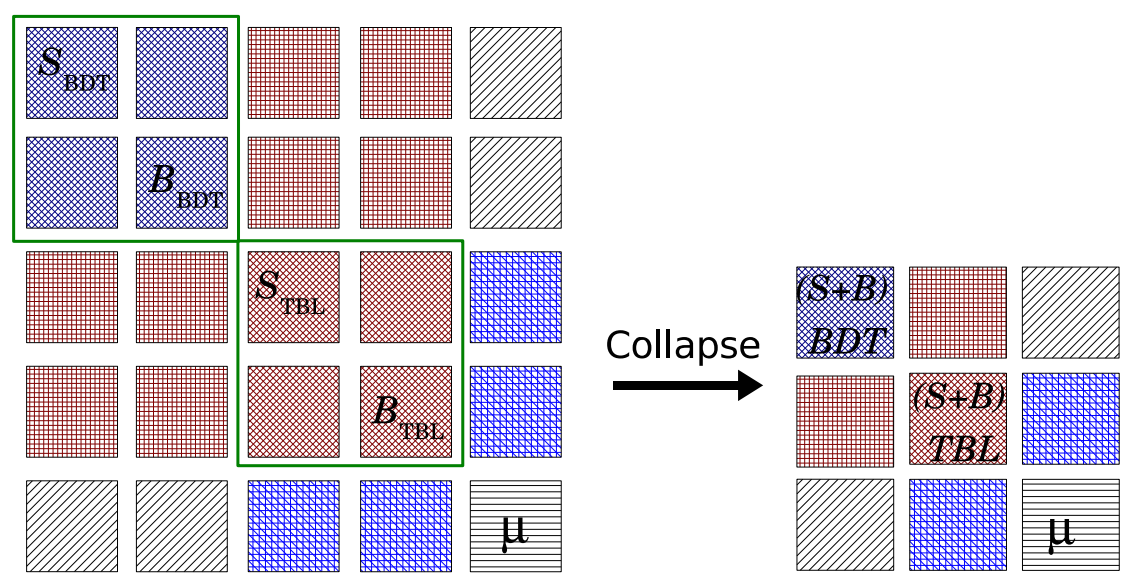

Extended error matrix

Figure 4.8: Illustration of the extended error matrix used in the combined BDT+TBL analysis. The matrix on the left is composed of a $\nu_{e}$ signal block $\left(S_{\mathrm{BDT}} / S_{\mathrm{TBL}}\right)$ and a $\nu_{e}$ background block $\left(B_{\mathrm{BDT}} / B_{\mathrm{TBL}}\right)$ for each of the two $\nu_{e}$ candidate samples, a $\nu_{\mu}$ CCQE block $(\mu)$, and their correlations.

As was mentioned before the BDT and TBL selection cuts form two largely independent sets of $\nu_{e}$ candidates with an important overlap between them. Inclusion of the shared events in the two samples into a combined analysis requires knowledge of the statistical correlations that are induced by the overlap in the energy distributions in the two samples. These correlations cause the appearance of off-diagonal elements to the statistical component of the error matrix $M_{i j}^{\text {stat }}$, which in the absence of such overlap would be diagonal.

Figure 4.9(b) shows a graphical representation of the part of the statistical error matrix that comes from data events appearing in both the BDT and the TBL energy distributions (overlapping events) and Figure 4.9(a) shows the remaining contribution from the events that only appear in one of them (non-overlapping events). The two analyses used different energy estimators, hence the spread shown in the blocks containing the correlations in Figure 4.9(b). The array resulting from the sum of the 


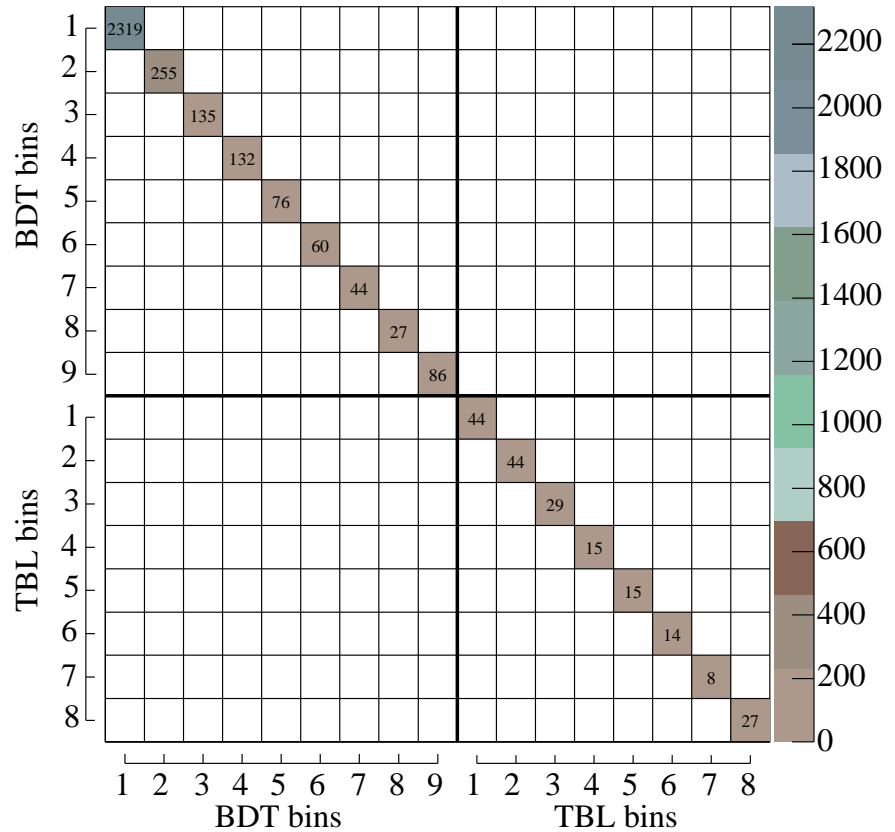

(a) Non-overlapping events..

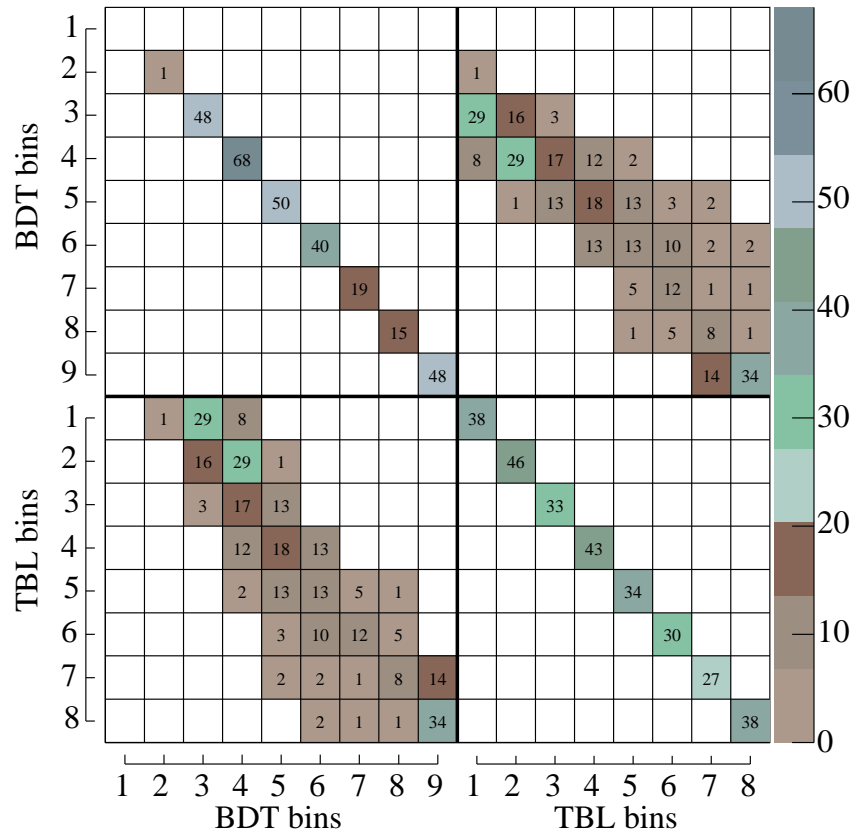

(b) Overlapping events.

Figure 4.9: The non-overlapping (a) and overlapping (b) $\nu_{e}$ candidate events from the data distributed over the reconstructed energy bins of the BDT and TBL analyses. Events overlap if they satisfy both selection criteria and have reconstructed energy within the boundaries of both analyses. 
arrays (a) and (b) in Figure 4.9 is the statistical error matrix that takes into account the overlap of events.

The handling of the statistical correlations was tested by performing a fit in which the same $\nu_{e}$ candidate sample (either BDT or TBL) was input twice together with the $\nu_{\mu}$ CCQE sample. The result of such experiments yielded identical results to that of using the $\nu_{e}$ sample once. This is shown in Figure 4.10 (4.11) for the BDT $(\mathrm{TBL}) \nu_{e}$ candidate distributions in a fit to a fake positive signal using only statistical uncertainties in the fit. The effect of ignoring the statistical correlations is equivalent to assume that we have twice the amount of data available, reducing the statistical uncertainty by a factor $\sim \sqrt{2}$. This incorrect assumption is visually obvious by comparing Figure 4.10(a) to Figure 4.10(b), where the latter gives smaller are contours, corresponding to a reduced total error. Once the statistical correlations are included, we recover the correct level of statistical uncertainty, which is manifest in the fact that Figure 4.10(c) yields identical regions as Figure 4.10(a). The corresponding comparisons apply for Figures 4.11(a), 4.11(b), and 4.11(c). Similar tests were performed in fits including the total errors and are shown in Appendix F.

As a second test, we performed fits in which the BDT and TBL $\nu_{e}$ candidate samples were input into the fit but the statistical correlations were turned off, resulting in an even stronger exclusion region which is expected from treating the two $\nu_{e}$ samples as independent increasing the statistics of the fit. The statistical correlations reduce this fictitious gain by incorporating the fact that only a fraction of the added events from the second sample are adding new information. 


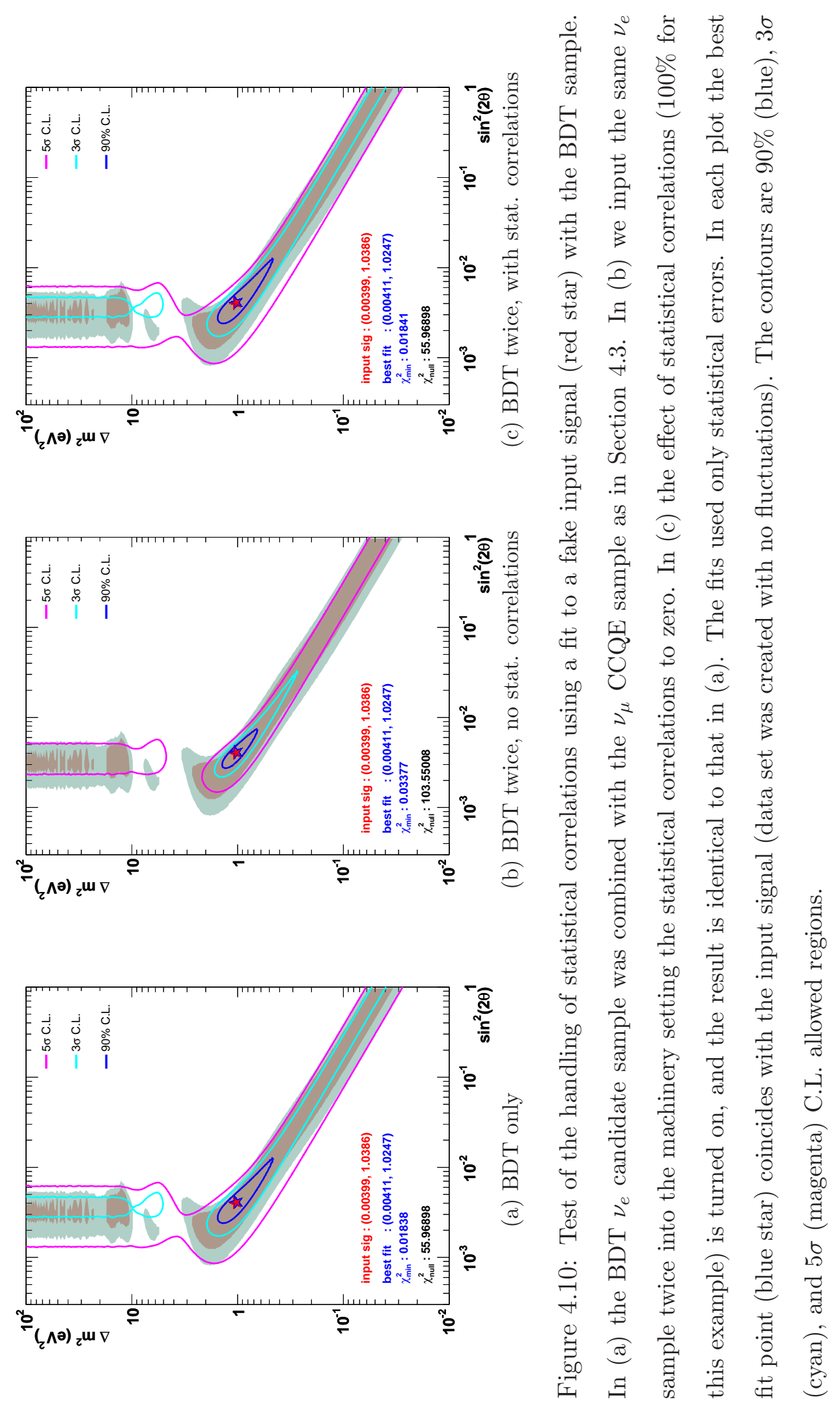




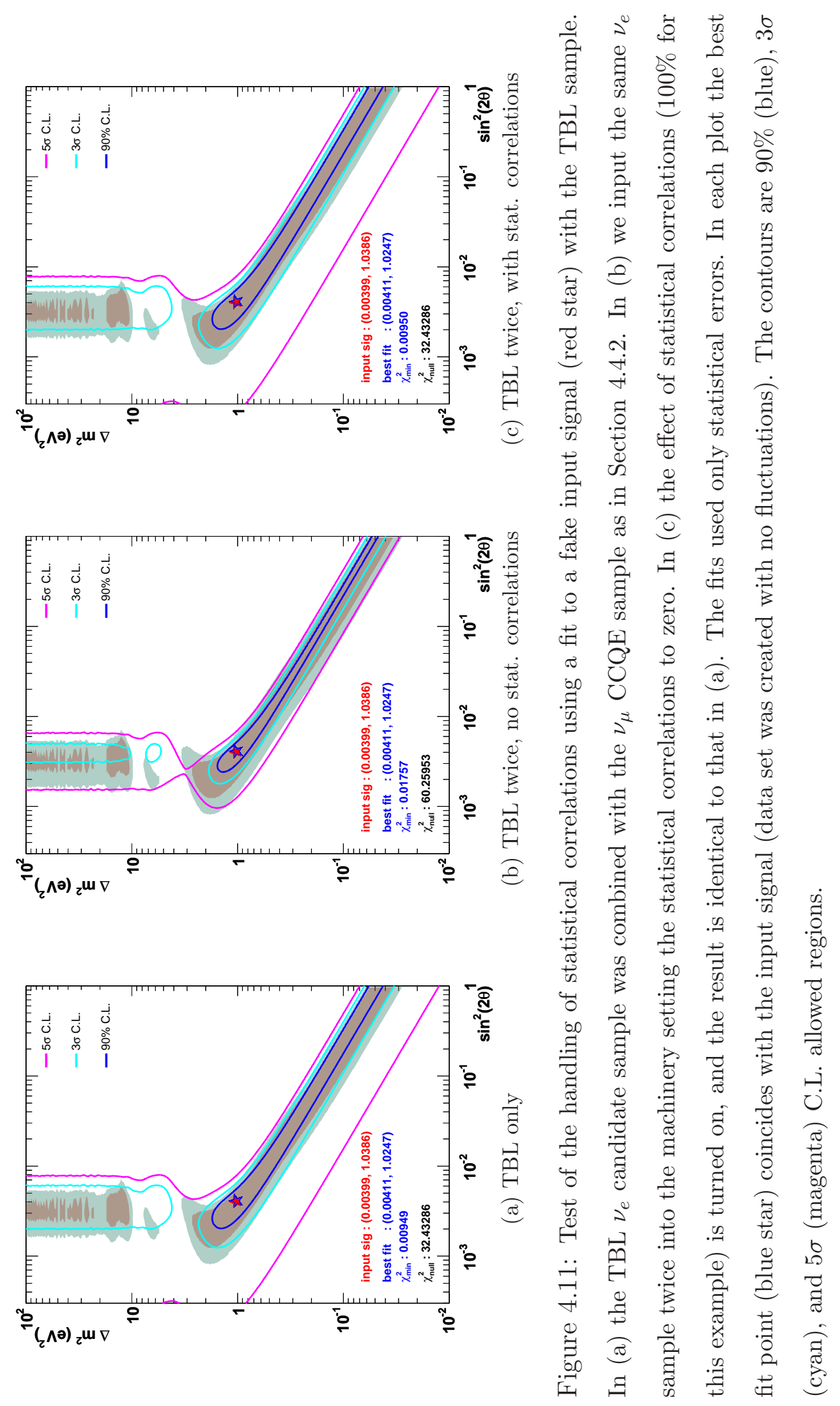




\section{Chapter 5}

\section{Results and Conclusions}

\section{$5.1 \quad$ Results}

To combine the BDT and TBL analyses we calculated the covariance of the bin contents of the three samples as described in 4.2.2, but with the bin index running freely across the three energy distributions: $\nu_{e} \mathrm{BDT}$ (signal and background), $\nu_{e} \mathrm{TBL}$ (signal and background), and $\nu_{\mu} \mathrm{CCQE}$, forming the $5 \times 5$-block matrix illustrated in Figure 4.8. The $3 \times 3$-block matrix resulting from the collapsing procedure described in Section 4.5 is then used to minimize the $\chi^{2}$ defined in Eq.(4.18) using the same minimization procedure as in Section 4.2.3. We used the smoothed O.M error estimate for this case in order to avoid introducing the pathologies observed in the BDT case.

In Figure 5.1(a) we compare the 90\% C.L. sensitivity to oscillations between the combined $\nu_{e} \mathrm{BDT}+\nu_{\mu} \mathrm{CCQE}$ fit of Section 4.3, the combined $\nu_{e} \mathrm{TBL}+\nu_{\mu} \mathrm{CCQE}$ fit of Section 4.4, and the fit using the three samples together. The blue (cyan) curve shows the result of combining the BDT $(\mathrm{TBA}) \nu_{e}$ candidate sample with the $\nu_{\mu}$ CCQE sample as was done in section 4.3 (4.4). The red curve shows the result of the present technique combining the two $\nu_{e}$ candidate samples with the $\nu_{\mu} \mathrm{CCQE}$ 
sample, which yields a significantly higher sensitivity to oscillations.

Figure 5.1(b) shows the corresponding comparison for the $90 \%$ C.L. limits to oscillations when the three types of constrained fits are performed on the MiniBooNE open data set. The combination of the two $\nu_{e}$ candidate samples gives a more powerful result for almost every value of $\Delta m^{2}$, although the improvement is less apparent in this case. Note that the specific fluctuations in the three data sets cause the limit obtained with the $\nu_{e} \mathrm{BDT}+\nu_{e} \mathrm{TBL}+\nu_{\mu} \mathrm{CCQE}$ fit to be worse than the $\nu_{e} \mathrm{BDT}+$ $\nu_{\mu} \mathrm{CCQE}$ or $\nu_{e} \mathrm{BDT}+\nu_{\mu} \mathrm{CCQE}$ limits for some values of $\Delta m^{2}$. While not ideal, this is a perfectly possible scenario, since the total data set used in each fit is different, adding new fluctuations that will affect the details of the final result in each case.

Figure 5.2 shows the energy distributions for the three samples used in the present fit. The corrections to the Monte Carlo prediction and the error bars are calculated using the transformations in Eq.(4.16), and Eq.(4.14), which are slightly different than those obtained for the two individual cases treated earlier.

Finally in Figure 5.3(b) we compare the result in Ref.[190] with the present fit combining the BDT and TBL $\nu_{e}$ candidate samples with the $\nu_{\mu}$ CCQE sample. The details of the oscillating portion of the limit are determined by how the fit responds to the fluctuations in the $\nu_{\mu}$ and $\nu_{e}$ data distributions ${ }^{1}$, and in this case the analysis does not improve the limit at the highest $\Delta m^{2}$ values. However, an increase of $\sim 10 \%-30 \%$ depending on the $\Delta m^{2}$ value, in the coverage of the region below $\Delta m^{2} \lesssim 1 \mathrm{eV}^{2}$ is achieved, which is a significant gain over the first publication.

Table 5.1 summarizes the results of a counting experiment combining the two samples. The number of events from the data in the reconstructed energy range used for each individual analysis is compared to the prediction and the significance of the

\footnotetext{
${ }^{1}$ The analysis in Ref.[190] used a different $\nu_{\mu}$ CCQE sample.
} 
Table 5.1: Comparison of the observed and predicted number of events in the energy range $300 \mathrm{MeV}<E_{\nu}^{Q E}-\mathrm{BDT}<1600 \mathrm{MeV}$ for the BDT analysis, and $475 \mathrm{MeV}<E_{\nu}^{Q E}-\mathrm{TBL}<1250$ $\mathrm{MeV}$ for the TBL analysis. The significances of the differences shown in parenthesis are calculated with the errors of Table 4.1 for the unconstrained case, and with the uncertainties resulting from the present technique for the constrained case. The overlap of the observed and expected events is shown in the last two rows.

\begin{tabular}{c|ccc}
\hline \hline & Observed & Unconstrained Prediction & Constrained Prediction \\
\hline \hline BDT & $970 \pm 31$ & $1066 \pm 266(-0.36 \sigma)$ & $1066 \pm 245,(-0.39 \sigma)$ \\
TBL & $378 \pm 19$ & $368 \pm 52(0.18 \sigma)$ & $368 \pm 41,(0.22 \sigma)$ \\
\hline \hline
\end{tabular}

Events distributed in both samples [overlap fraction in \%]:

\begin{tabular}{c|ccc|cc}
\hline \hline & BDT only & TBL only & BDT \& TBL & BDT Total & TBL Total \\
\hline Observed & 748 & 156 & 222 & $970[22.9 \%$ ov. $]$ & $378[58.7 \%$ ov.] \\
Expected & 853 & 155 & 213 & $1066[20.0 \%$ ov. $]$ & $368[57.9 \%$ ov.] \\
\hline \hline
\end{tabular}

deviation is calculated as

$$
N_{\sigma}=\frac{N_{\mathrm{data}}-N_{\mathrm{pred}}}{\sqrt{\sigma_{\mathrm{stat}}^{2}+\sigma_{\mathrm{sys}}^{2}}}
$$

Notice that the total systematic uncertainty in each case is reduced with the combined technique, and the difference in the total number of events is within one standard deviation after the constraint. 


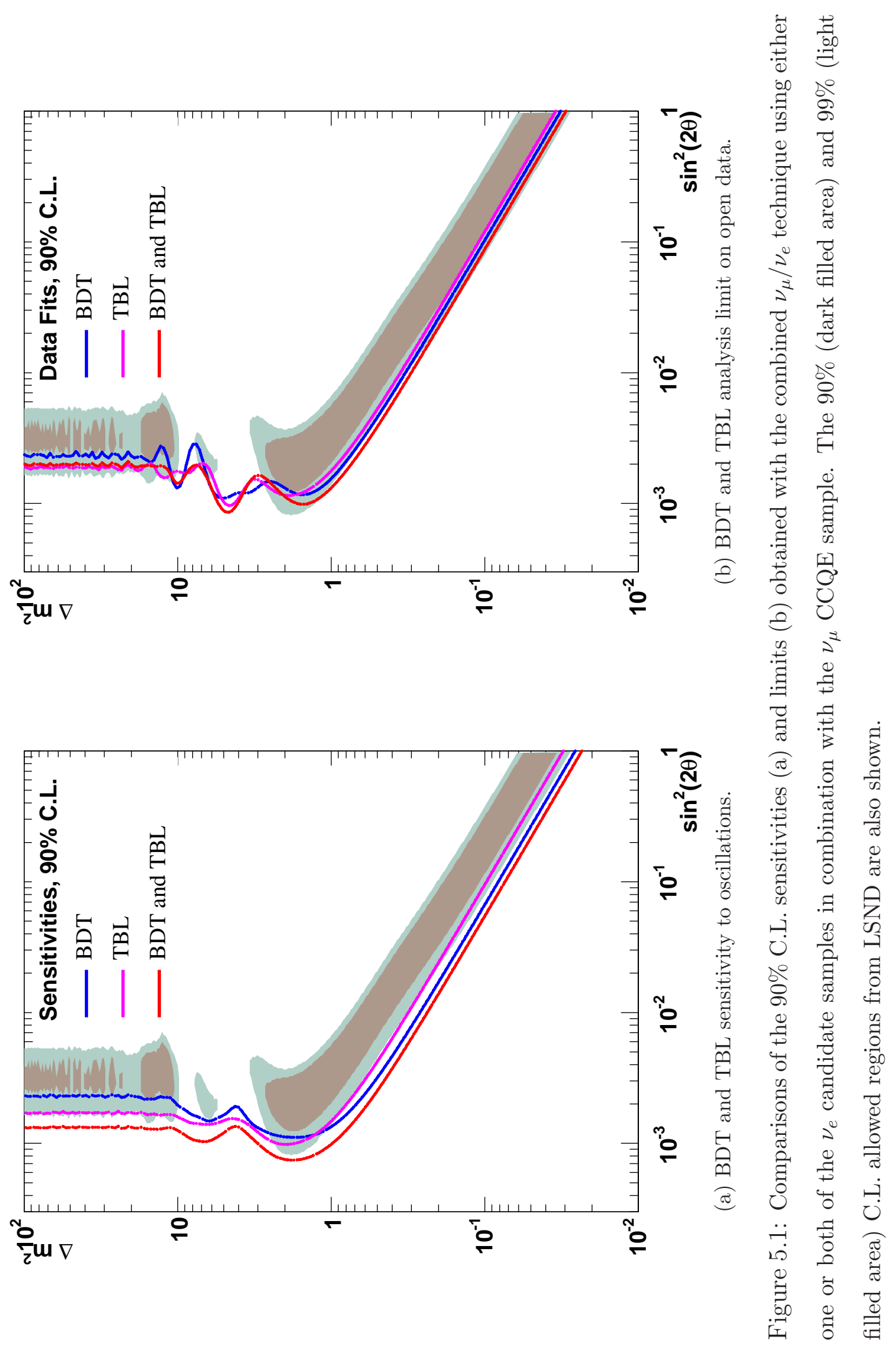




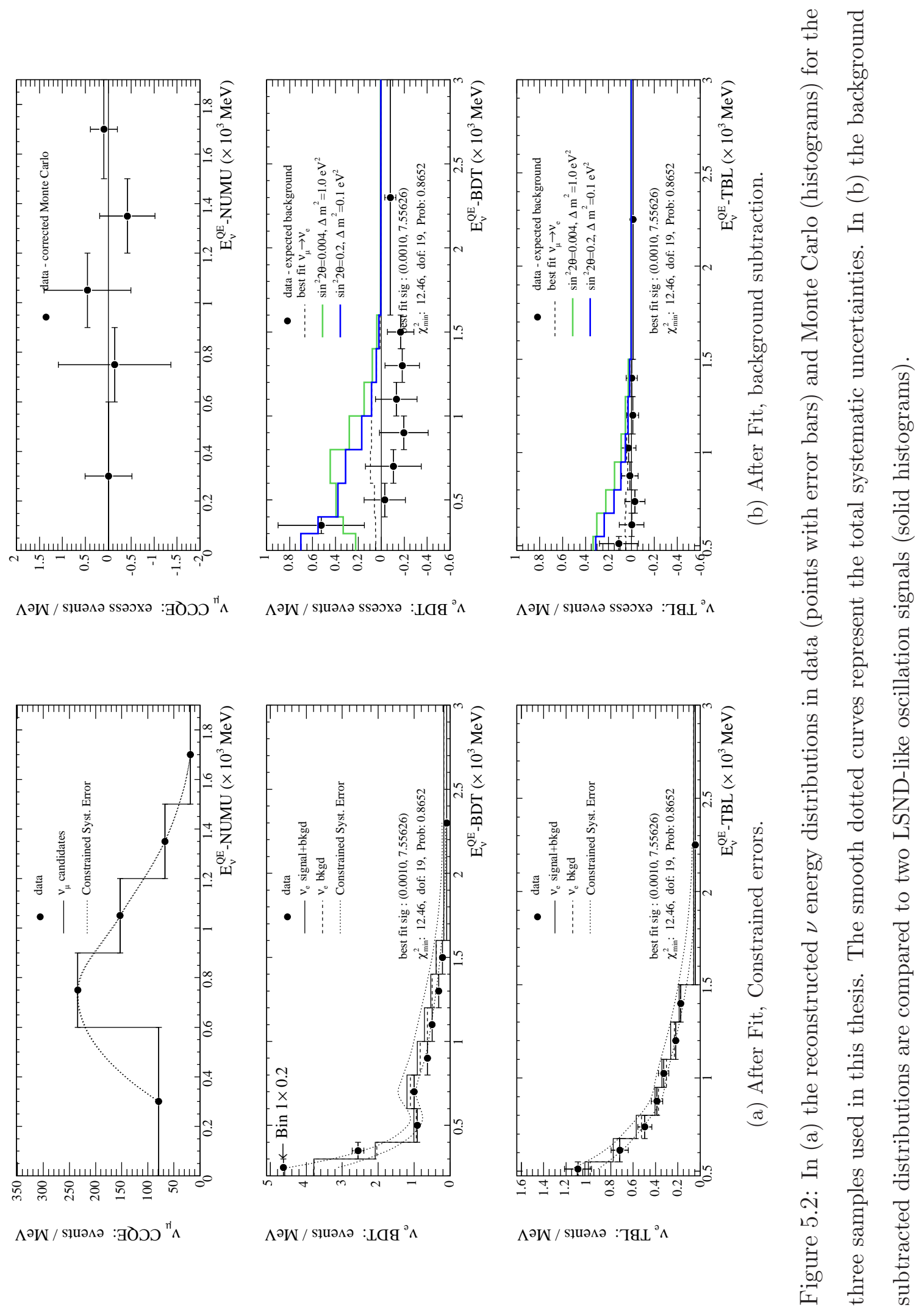




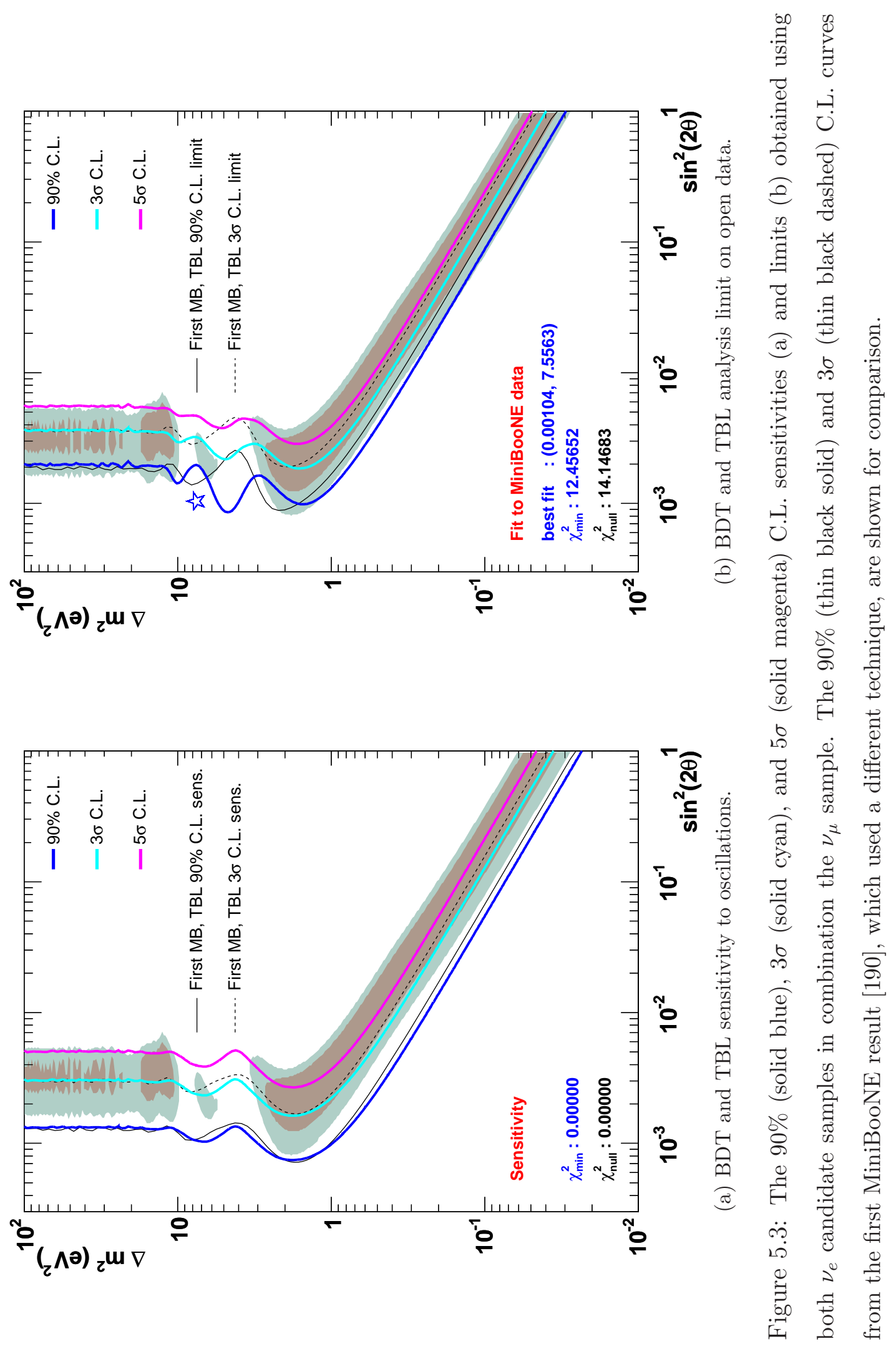




\subsection{Conclusions}

Non-zero neutrino masses and mixings have been established by observations of solar, atmospheric, reactor, and accelerator-based neutrino oscillations experiments over the last two decades (see Section 1.4). These observations seem to be consistent with the three-neutrino picture of the Standard Model of elementary particles, except for the result of the LSND experiment at Los Alamos. If due to oscillations, the LSND observation would have important implications for the Standard Model, requiring the existence of at least one sterile neutrino.

First results from the MiniBooNE experiment [190] at Fermilab, have ruled out the possibility that the LSND observation be due to a simple two-neutrino oscillations model as was previously claimed. MiniBooNE used two oscillations analyses which differed in reconstruction algorithms, sample selection cuts, methods to constrain systematic errors, and the oscillations fit technique. Both analyses gave a consistent answer.

In this thesis we have described the technique used to obtain the result of the cross check analysis appearing in the first MiniBooNE publication [190] (the BDT ${ }^{2}$ analysis), in which a high statistics sample of $\nu_{\mu}$ events is introduced in the definition of a $\chi^{2}$ statistic minimized to fit a $2 \nu$ oscillations model with the aim to constrain the systematic uncertainties on the predicted number of $\nu_{e}$ events. This $\chi^{2}$ uses the reconstructed energy distributions of the $\nu_{\mu}$ and $\nu_{e}$ events simultaneously, with only the $\nu_{e}$ events being affected by the oscillations model parameters: $\Delta m^{2}$ and $\sin ^{2} 2 \theta$. All sources of uncertainty on the underlying simulation parameters were propagated to calculate the covariance matrix of the contents of the $\nu_{\mu}$ and $\nu_{e}$ reconstructed energy bins. The final covariance matrix contains all systematic and statistical errors

${ }^{2}$ Boosted Decision Tree. From the particle identification technique used to isolate the sample. Described in Section 3.5.2. 
as well as the correlations between the various $\nu_{\mu}$ and $\nu_{e}$ reconstructed energy bins.

The approach used in this thesis is significantly different from the one used for the main result in Ref.[190], nonetheless, we obtain comparable results to those presented therein when we use the $\nu_{e}$ sample from the $\mathrm{TBL}^{3}$ analysis cuts in our combined $\nu_{\mu} / \nu_{e}$ fit technique, and treat the systematic errors consistently with Ref.[190].

An extension of the combined $\nu_{\mu} / \nu_{e}$ fit technique was developed to include a second sample of $\nu_{e}$ events in the definition of the $\chi^{2}$, and was used in a combined analysis of the $\nu_{e}$ sample from the first MiniBooNE publication (the TBL $\nu_{e}$ sample), the BDT $\nu_{e}$ sample, and the high statistics $\nu_{\mu}$ sample. This extension required appropriate handling of the non-negligible event overlap between the two $\nu_{e}$ samples, which induces additional correlations between their reconstructed energy bins. These correlations were included in the definition of the statistical component of the total error matrix used in the $\chi^{2}$ and appear as off-diagonal elements connecting the energy bins of both $\nu_{e}$ samples $^{4}$. This allowed us to make use of all the available events passing both $\nu_{e}$ selection criteria.

We compared the sensitivities to $2 \nu$ oscillations in the three scenarios studied in this work:

$$
\text { 1) } \nu_{e} \mathrm{BDT}+\nu_{\mu}, \text { 2) } \nu_{e} \mathrm{TBL}+\nu_{\mu}, \text { 3) } \nu_{e} \mathrm{BDT}+\nu_{e} \mathrm{TBL}+\nu_{\mu}
$$

and found that the combination of both $\nu_{e}$ candidate samples with the $\nu_{\mu}$ sample (number 3 above) yields stronger sensitivities than the use of either one of them separately. The same is true in general for the limits to oscillations extracted from fits to the MiniBooNE data, although specific fluctuations of the three data sets cause the limit in scenario number 3 above to be worse than those in scenarios 1 or 2 for

\footnotetext{
${ }^{3}$ Track Based Likelihood particle identification technique described in Section 3.5.1.

${ }^{4}$ In the absence of event overlap the statistical error matrix is diagonal with the number of events in each bin along the diagonal.
} 
some values of $\Delta m^{2}$. When compared to the result in Ref.[190] the present technique yields a stronger limit to oscillations in the region of $\Delta m^{2} \lesssim 1 \mathrm{eV}^{2}(\sim 10 \%-30 \%$ increase in coverage depending on $\Delta m^{2}$ ).

This result strengthens the statement that the MiniBooNE data rules out the interpretation of the LSND result as due to $\bar{\nu}_{\mu} \rightarrow \bar{\nu}_{e}$ oscillations described under the standard $L / E$ dependence of oscillations and the usual CP and CPT invariance assumptions. 


\section{Bibliography}

[1] W. Pauli, Unpublished letter to the Physical Society of Tubingen, dated December 4, 1930; reproduced in L. .M. Brown, Physics Today, 31, No. 9, 23 (1978).

[2] Proc. Roy. Soc., A136,692 (1932).

[3] E. Fermi, Z. Physik, 88, 161 (1934).

[4] C. L. Cowan Jr., F. Reines, F. B. Harrison, H. K. Krusse, A. D. McGuire, Science, 124, 103 (1956).

[5] G. Danby, J-M. Gaillard, K. Goulianos, L. M. Lederman, N. Mistry, M. Schwartz, and J. Steinberger Phys. Rev. Lett. 9, 36 (1962).

[6] M. L. Perl et al., Phys. Rev. Lett. 35, 1489 (1975).

[7] F. Abe et al., Phys. Rev. Lett. 74, 2626 (1995)

S. Abachi et al., Phys. Rev. Lett. 74, 2632 (1995).

[8] B. Pontecorvo, Zh. Eksp. Teor. Fiz. 34 247, (1957) [Sov. Phys. JETP 7172 , 1958].

[9] T. D. Lee, R. Oehme, and C. N. Yang, Phys. Rev. 106, 340 (1957);

T. D. Lee and C. S. Wu, Ann. Rev. Nucl. Sci. B 247511 (1966); 
J. Lowe, B. Bassalleck, H. Burkhardt, T. Goldman, A. Rusek and G. J. Stephenson, Jr., Phys. Lett. B384, 288 (1996).

[10] Z. Maki,M. Nakagawa and S. Sakata, Prog. Theor. Phys. 28 870, (1962).

[11] W.-M. Yao et al., Journal of Physics G 33, 1 (2006), [http://pdg.1bl.gov/].

[12] B. Kayser, Phys Rev. D 24, 110 (1981);

F. Bohem and P. Vogel, Physics of Massive Neutrinos (Cambridge University Press, Cambridge, 1987), pp. 87;

C. Giunti, C. W. Kim, and U. Lee, Phys. Rev. D 44, 3635 (1991);

J. Rich, Phys. Rev. D 48, 4318 (1993);

H. Lipkin, Phys. Lett. B348, 604 (1995)

W. Grimus and P. Stockinger, Phys. Rev. D 54, 3414, (1996)

T. Goldman, hep-ph/9604357

Y. Grossman and H. Lipkin, Phys. Rev. D 55, 2760 (1997);

W. Grimus, S. Mohanty, and P. Stockinger, in Proc. of the 17th Int. Workshop on Weak Interactions and Neutrinos, eds. C. Dominguez and R. Violler (World Scientific, Singapore, 2000), pp. 355.

[13] C. Giunti, C. W. Kim, Found. Phys. Lett. 14, 213-229 (2001).

[14] D. E. Groom et al., Eur. Phys. J. C15, 1 (2000), WWW page: [http://pdg.1bl.gov].

[15] Y. Farzan and A. Y. Smirnov, Phys. Lett. B557, 224 (2003).

[16] F. T. Avignone, III, S. R. Elliott, J. Engel,. LA-UR-07-3577, arXiv:0708.1033 [nucl-ex] (2007).

[17] A. Barabash, Czech. J. Phys. 56437 (2006). 
[18] V. Rodin and A. Faessler, Prog. Part.Nucl. Phys. 57, 226 (2006),

C. E. Aalseth, et al., Phys. Rev. D. 65, 092007 (2002).

H. Klapdor-Kleingrothaus, et al., Eur. Phys. J. A 12, 147 (2001).

[19] C. Quigg, Gauge Theories of the Strong, Weak, and Electromacnetic Interactions, The Benjamin/Cummings Publishing Company (1983).

[20] F. Bohem and P. Vogel, Physics of Massive Neutrinos, second edition, Cambridge University Press (1992).

[21] B. Kayser, F. Gibrart-Debu, and F. Perrier, The Physics of Massive Neutrinos, World Scientific (1992).

[22] C.W. Kim and A. Pevsner, Neutrinos in Physics and Astrophysics, Harwood Academic Publishers (1993).

[23] R. Mohapatra and P. Pal, Massive Neutrinos in Physics and Astrophysics, Third Ed., World Scientific (2004).

[24] P. Renton, Electroweak Interactions, Cambridge University Press (1990).

[25] J. Bonn et al., Nucl. Phys. Proc. Suppl. 91 (2001) 273.

[26] V. M. Lobashev et al., Nucl. Phys. Proc. Suppl. 91, 280 (2001).

[27] A. Osipowicz et al. [KATRIN Collaboration], arXiv:hep-ex/0109033.

[28] O. Host, O. Lahav, F. B. Abdalla, K. Eitel, Submitted to Phys.Rev.D, e-Print: arXiv:0709.1317 [hep-ph].

[29] T. Bowles and R. G. Hamish, Tritium Beta Decay and the search for Neutrino Mass, Los Alamos Science, 25 (1997).

[30] N. Angelov et al., nucl-ex/0605002 (2006). 
[31] K. Assamagan et al., Phys. Rev. D53, 6065-6077 (1996).

[32] K. Assamagan et al., Phys. Lett. B434, 158-162 (1998).

[33] R. Barate et al. (ALEPH), Eur. Phys. J. C2, 395-406 (1998).

[34] J. Orloff, A. Rozanov, C. Santoni, Phys. Lett. B550, 8-15 (2002).

[35] P. Moller, J. R. Nix, W. D. Myers, and W. J. Swiatecki, Atomic Data Nucl. Data Tables 59, 185-381 (1995), LA-UR-93-3083, Nuclear Ground-State Masses and Deformations [http://t2.lanl.gov/data/astro/molnix96/molnix.html].

[36] G. Audi et al., Nucl. Phys. A729, 337-676 (2003) .

[37] C. Athanasopoulos et al., Phys. Rev. Lett. 75, 2650 (1995).

[38] C. Athanasopoulos et al., Phys. Rev. C. 54, 2685 (1996);

C. Athanasopoulos et al., Phys. Lett. 77, 3082 (1996).

[39] C. Athanasopoulos et al., Phys. Rev. Lett. 81, 1774 (1998);

C. Athanasopoulos et al., Phys. Rev. C. 58, 2489 (1998).

[40] A. Aguilar et al. [LSND Collaboration], Phys. Rev. D 64, 112007 (2001) [arXiv:hep-ex/0104049].

[41] M. C. Gonzalez-Garcia, arXiv:0704.1800 [hep-ph] (2007).

[42] J. N. Bahcall, A. M. Serenelli and S. Basu, Astrophys. J. 621 (2005) L85 [arXiv:astro-ph/0412440].

[43] R. J. Davis, D. S. Harmer and K. C. Hoffman, Phys. Rev. Lett. 201205 (1968).

[44] B. T. Cleveland et al., Astrophys. J. 496505 (1998). 
[45] J. N. Abdurashitov et al. [SAGE Collaboration], J. Exp. Theor. Phys. 95181 (2002) [Zh. Eksp. Teor. Fiz. 122211 (2002)] [arXiv:astro-ph/0204245].

[46] W. Hampel et al. [GALLEX Collaboration], Phys. Lett. B 447127 (1999).

[47] M. Altmann et al. [GNO Collaboration], Phys. Lett. B 616174 (2005). [arXiv:hep-ex/0504037].

[48] Y.!Fukuda et al. [Kamiokande Collaboration], Phys. Rev. Lett. 771683 (1996).

[49] M. B. Smy et al. [Super-Kamiokande Collaboration], Phys. Rev. D 69011104 (2004) [arXiv:hep-ex/0309011].

[50] J. N. Bahcall, N. A. Bahcall and G. Shaviv, Phys. Rev. Lett. 20 (1968) 1209. J .N. Bahcall and R. Davis, Science 191 (1976) 264.

[51] Q. R. Ahmad et al. [SNO Collaboration], Phys. Rev. Lett. 87 (2001) 071301 [arXiv:nucl-ex/0106015].

[52] Q. R. Ahmad et al. [SNO Collaboration], Phys. Rev. Lett. 89, 011301 (2002) [arXiv:nucl-ex/0204008].

[53] Q. R. Ahmad et al. [SNO Collaboration], Phys. Rev. Lett. 89, 011302 (2002) [arXiv:nucl-ex/0204009].

[54] S. N. Ahmed et al. [SNO Collaboration], Phys. Rev. Lett. 92 (2004) 181301 [arXiv:nucl-ex/0309004].

[55] B. Aharmim et al. [SNO Collaboration], Phys. Rev. C 72 (2005) 055502 [arXiv:nucl-ex/0502021].

[56] L. Wolfenstein, Phys. Rev. D 17 (1978) 2369.

S. P. Mikheev and A. Y. Smirnov, Sov. J. Nucl. Phys. 42, 913 (1985) [Yad.Fiz. 42, $1441(1985)]$. 
[57] C. Arpesella et al. [Borexino Collaboration], arXiv:0708.225[astro-ph]

[58] F. Reines et al., Phys. Rev. Lett. 15 (1965) 429.

[59] C. V. Achar et al., Phys. Lett. 18 (1965) 196.

[60] K. Daum et al. [Frejus Collaboration.], Z. Phys. C 66 (1995) 417.

[61] M. Aglietta et al. [NUSEX Collaboration], Europhys. Lett. 8 (1989) 611.

[62] R. Becker-Szendy et al., Phys. Rev. D 46 (1992) 3720.

[63] Y. Fukuda et al. [Kamiokande Collaboration], Phys. Lett. B 335 (1994) 237.

[64] G. D. Barr, T. K. Gaisser, P. Lipari, S. Robbins and T. Stanev, Phys. Rev. D 70 (2004) 023006 [arXiv:astro-ph/0403630].

M. Honda, T. Kajita, K. Kasahara and S. Midorikawa, Phys. Rev. D 70 (2004) 043008 [arXiv:astro-ph/0404457].

G. Battistoni, A. Ferrari, T. Montaruli and P. R. Sala, Astropart. Phys. 19 (2003) 269 [Erratum-ibid. 19 (2003) 291] [arXiv:hep-ph/0207035].

Y. Liu, L. Derome and M. Buenerd, Phys. Rev. D 67 (2003) 073022 [arXiv:astro$\mathrm{ph} / 0211632]$.

J. Wentz, I. M. Brancus, A. Bercuci, D. Heck, J. Oehlschlager, H. Rebel and B. Vulpescu, Phys. Rev. D 67 (2003) 073020 [arXiv:hep-ph/0301199].

[65] Y. Fukuda et al. [Super-Kamiokande Collaboration], Phys. Rev. Lett. 81 (1998) 1562 [arXiv:hep-ex/9807003].

[66] Y. Ashie et al. [Super-Kamiokande Collaboration], Phys. Rev. D 71 (2005) 112005 [arXiv:hep-ex/0501064].

[67] J. Hosaka et al. [Super-Kamiokande Collaboration], Phys. Rev. D 74 (2006) 032002 [arXiv:hep-ex/0604011]. 
[68] Talk by P.J. Litchfield at The XXII International Conference on Neutrino Physics, Santa Fe, New Mexico, June 13-19 (2006).

[69] M. C. Sanchez et al. [Soudan 2 Collaboration], Phys. Rev. D 68, 113004 (2003) [arXiv:hep-ex/0307069].

[70] M. Ambrosio et al. [MACRO Collaboration], Phys. Lett. B 517, 59 (2001) [arXiv:hep-ex/0106049].

[71] S. Fukuda et al. [Super-Kamiokande Collaboration], Phys. Rev. Lett. 85, 3999 (2000) [arXiv:hep-ex/0009001].

[72] K. Abe et al. [Super-Kamiokande Collaboration], Phys. Rev. Lett. 97, 171801 (2006) [arXiv:hep-ex/0607059].

[73] G. Zacek et al. [CALTECH-SIN-TUM Collaboration], Phys. Rev. D 34, 2621 (1986).

[74] G. S. Vidyakin et al., JETP Lett. 59 (1994) 390 [Pisma Zh. Eksp. Teor. Fiz. 59, $364(1994)]$.

[75] Y. Declais et al., Nucl. Phys. B 434, 503 (1995).

[76] M. Apollonio et al. [CHOOZ Collaboration], Phys. Lett. B 466 (1999) 415 [arXiv:hep-ex/9907037].

[77] A. Piepke et al. [Palo Verde Collaboration], Prog. Part. Nucl. Phys. 48, 113 (2002) .

[78] A. Piepke et al. [KamLAND Collaboration], Nucl. Phys. Proc. Suppl. 91, 99 (2001) . 
[79] K. Eguchi et al. [KamLAND Collaboration], Phys. Rev. Lett. 90, 021802 (2003) [arXiv:hep-ex/0212021].

[80] T. Araki et al. [KamLAND Collaboration], Phys. Rev. Lett. 94, 081801 (2005) [arXiv:hep-ex/0406035].

[81] J. Shirai [for the KamLAND collaboration], Prepared for Workshop on Neutrino Oscillation Physics (NOW 2006), Otranto, Lecce, Italy, 9-16 Sep 2006, Nucl.Phys.Proc.Suppl.168:77-83 (2007)

[82] S. Kopp, Phys. Rep. 439, 102-159 (2006).

[83] K. Nishikawa, Nucl. Phys. Proc. Suppl. 59, 289 (1997) .

[84] E. Ables et al. [MINOS Collaboration], FERMILAB-PROPOSAL-P-875 (1995).

[85] S. H. Ahn et al. [K2K Collaboration], Phys. Lett. B 511, 178 (2001) [arXiv:hepex/0103001].

[86] E. Aliu et al. [K2K Collaboration], Phys. Rev. Lett. 94, 081802 (2005) [arXiv:hep-ex/0411038].

[87] D. G. Michael et al. [MINOS Collaboration], Phys. Rev. Lett. 97, 191801 (2006) [arXiv:hep-ex/0607088].

[88] The MINOS Collaboration, FERMILAB-PUB-07-413, 13pp (2007); arXiv:0708.1495[hep-ex].

[89] A. G. Cocco [OPERA Collaboration], Nucl. Phys. Proc. Suppl. 85, 125 (2000).

[90] J. Marteau et al. [OPERA Collaboration], [arXiv:hep-ex/07061699 (2007) R. Acquafredda et al. [OPERA Collaboration], New J. Phys. 8, 303 (2006) [arXiv:hep-ex/0611023]. 
[91] F. Dydak et al. [CDHS Collaboration], Phys. Lett. B 134, 281 (1984).

[92] L. Borodovsky et al. [E776 Collaboration], Phys. Rev. Lett. 68, 274 (1992).

[93] L. A. Ahrens et al. [E734 Collaboration], Phys. Rev. D 36, 702 (1987).

[94] B. Armbruster et al. [KARMEN Collaboration], Phys. Rev. D 65, 112001 (2002) [arXiv:hep-ex/0203021].

[95] N. Ushida et al. [E531 Collaboration], Phys. Rev. Lett. 57, 2897 (1986).

[96] A. Romosan et al. [CCFR/NuTeV Collaboration], Phys. Rev. Lett. 78, 2912 (1997) [arXiv:hep-ex/9611013].

[97] D. Naples et al. [CCFR/NuTeV Collaboration], Phys. Rev. D 59, 0311014 (1999) [arXiv:hep-ex/9809023].

[98] K. S. McFarland et al., Phys. Rev. Lett. 75, 3993 (1995) [arXiv:hepex/9506007].

[99] E. Eskut et al. [CHORUS Collaboration], Phys. Lett. B497, 8 (2001).

[100] P. Astier et al. [NOMAD Collaboration], Nucl. Phys. B611, 3 (2001) [arXiv:hep-ex/0106102].

[101] E. D. Church, K. Eitel, G. B. Mills, and M. Steidl, Phys. Rev. D 66, 013001 (2002)

[102] Includes contributions from $\bar{\nu}_{\mu} C \rightarrow \mu^{+} n X$ and $\nu_{\mu} C \rightarrow \mu^{-} n X$

[103] (Marry Bishai, Private Communication.)

[104] 1 mill $=0.00254 \mathrm{~cm}=1 / 1000 \mathrm{in}$.

[105] L. Bugel, M. Sorel, MiniBooNE Technical Note \#34. 
[106] J. A. Green, Documentation of the MiniBoonE Analysis Framework, Latency Filter. http://www-boone.fnal.gov/software_and_analysis/ current_framework/doc/index.html

[107] D. Finley, "Relative Timing of Neutrinos from the Fermilab Debuncher and the MiniBooNE Beam", MiniBooNE Memo \#4.

[108] Ludox ${ }^{\circledR}$ Colloidal Silica, is a registered trademark of Grace Davison. http://www.gracedavison.com/products/ludox/overview.htm

[109] R. Imlay et al., NIM, 2006, in preparation; M. O. Wascko, private communication.

[110] R. Imlay, B. Metcalf, S. Ouedraogo, M. Sung, and M. Wascko, MiniBooNE Technical Note \#106.

[111] I. Stancu, MiniBooNE Technical Note \#63.

[112] B. Rossi, High-Energy Particles, Prentice Hall Series (1965).

[113] I. Stancu. MiniBooNE Technical Note \#21.

[114] Y. Liu and I. Stancu, MiniBooNE Technical Note \#126.

[115] M. Tzanov, MiniBooNE Technical Note \#204.

[116] I. Stancu it et al., Technical Design Report for the 8 GeV Beam, http://www-boone.fnal.gov/publicpages/8gevtdr_2.0.ps.gz (2001).

[117] I. Stancu it et al., The MiniBooNE Detector Technical Design Report, http://www-boone.fnal.gov/publicpages/detector_tdr.ps.gz (2001).

[118] E. D. Zimmerman et al., LMC Technical Design Report. 
[119] T. Kobilarcik, J. Devaoy, and C. Moore, Automatic Beam Correction, proceedings of the 2003 Particle Accelerator Conference (2003).

[120] P. Lipari, M. Lusignoli, and F. Sartogo, Phys. Rev. D 74, 4384 (1995).

[121] J. R. Sanford and C. L. Wang, BNL AGS internal reports BNL11299 and BNL11479 (1967).

[122] C. L. Wang, Phys. Rev. Lett. 251068 (1970).

[123] S. Agostinelli et al. [GEANT4 Collaboration], Nucl. Instrum. Meth. A506 250 $(2003)$.

[124] http://wwasd.web.cern.ch/geant4/G4UsersDocuments/Overview/html

[125] N. V. Mokhov, FERMILAB-FN0628 (1995).

[126] N. V. Mokhov et al., FERMILAB-CONF-04-053-AD, (2004); Radiat. Prot. Dosim. 116, 99 (2005); e-Print: nucl-th/0404084.

[127] R. P. Feynman, Phys. Rev. Lett. 23, 1415 (1969).

[128] I. Chemakin et al. [E910 Collaboration] Submitted to Phys. Rev. C, arXiv:0707.2375[nucl-ex].

[129] M. G. Catanesi et al. [The HARP Collaboration], Nucl. Phys. B 732, 1-45 (2006).

[130] D. Schmitz, private comunication.

[131] M. H. Shaevitz, MiniBooNE Technical Note \#209.

[132] M. H. Shaevitz, MiniBooNE Technical Note \#188.

[133] T. Abbott et al., Phys. Rev. D45, 3906 (1992). 
[134] J. V. Allaby et al., CERN 70-12Nuclear Physics Division April 23, 1970

[135] D. Dekkers et al., Phys. Rev. 137, B962 (1965).

[136] R. A. Lundy et al., Phys. Rev. Lett. 14, 504 (1965).

[137] G. J. Marmer et al., Phys. Rev.; 179, No. 5; (1969).

[138] P. A. Piroue et al., Phys. Rev.; 148, No. 6; (1966).

[139] T. Eichten et al., Nucl. Phys. B44,333-343 (1972).

[140] Aleshin et al., ITEP-77-80 (1977).

[141] Vorontsov et al., ITEP-88-11 (1988).

[142] Analysis of the E910 data by J. Link. For some details of the experiment see: I. Chemakin et al. [E910 Collaboration], Nucl. Phys. A 639, 407 (1998).

[143] F. Abe et al., Phys. Rev. D 36, 1302 (1987).

[144] H. A. Tanaka, MiniBooNE Technical Note \#186.

[145] H. A. Tanaka and M. Wilking, MiniBooNE Technical Note \#206.

[146] R. J. Glauber, in Lectures in Theoretical Physics, edited by W. E. Britten et al. (Intersceince, New York, 1959), Volume I.

[147] A. Fasso, A. Ferrari, J. Ranft, and P.R. Sala, CERN-2005-10 (2005), INFN/TC_05/11, SLAC-R-773.

[148] D. Casper, Nucl. Inst. Proc. Suppl. 112, 161 (2002); [arXiv:hep-ph/0208030].

[149] Z. Djurcic, J. M. Link, J. L. Raaf, H. A. Tanaka, R. B. Patterson, G. P. Zeller, MiniBooNE Technical Note \#194. 
[150] D. Rein and L. M. Sehgal, Annals Phys. 133, 79 (1981).

[151] E. A. Hawker, Nucl. Phys. Proc. Suppl. 139, 250 (2005); W. Krenz et al., Nucl. Phys. B135, 45 (1978).

[152] S. Nakayama et.al, Phys. Lett. B619, 255 (2005).

[153] H. Faissner et al., Phys. Lett B125, 230 (1983).

[154] R. Brun et al., GEANT3, CERN-DD/EE/84-1.

[155] H. O. Meyer, MiniBooNE Technical Note \#90.

[156] P. Meyers, MiniBooNE Technical Note \#135.

[157] C. Zeitnitz and T. A. Gabriel, Nucl. Instrum. Meth. A349 106 (1994).

[158] D. Toptygin, JHU Fluorescence Reports 1-7. B .C. Brown et al., IEEE Nucl. Sci. Symp. Conf. Rec. 1, 652 (2004).

[159] B. C. Brown, S. Maza, adn A. Pla-Dalmau, MiniBooNE Technical Note \#145.

[160] H.-J. Yang, MiniBooNE Technical Note \# 203.

[161] Kendall Mahn, Private communication.

[162] "Non Uniform Random Variate Generation", Luc Devroye Springer Verlag, NY, 1986, p.p. 563-567.

[163] C.M. Mauer, Ph.D. thesis, SUNY at Stony Brook, 2002; S. Nakayama, Nucl. Phys. B, Proc. Suppl. 139, 41 (2005).

[164] S. K. Singh, et al., Phys. Rev. Lett, 96, 24181 (2006).

[165] V. Nguyen, Talk presented at the Ninth International Workshop of Neutrino Factories, SuperBeams and BetaBeams, Okayama Japan, Aug 6-11 (2007). 
[166] A. Aguilar-Arevalo, "Multisims and Linearity Assumptions", BooNE Technical Note \#200.

[167] A. Aguilar-Arevalo, S. Brice, and M. H. Shaevitz, BooNE Technical Note \#212.

[168] E. M. Laird, R. B. Patterson, H. A. Tanaka, BooNE Technical Note \#222.

[169] G. J. Feldman and R. D. Cousins, Phys. Rev. D 57, 3873 (1988).

[170] T. Schwetz, arXiv:hep-ph/0612223v1 (2006).

[171] These numbers were calculated using the CERNLIB routine CHISIN.

[172] NIST/SEMATECH, e-Handbook of Statistical Methods, http://www.itl.nist.gov/div898/handbook.

[173] As defined in the Handbook of Mathematical Functions by Abramowitz and Stegun, formula 6.5.1 on page 260 .

[174] A. Aguilar-Arevalo, BooNE Technical Note \#223, and Adenda 1 and 2.

[175] A. A. Aguilar-Arevalo, M. Wilking, MiniBooNE Technical Note \#224.

[176] Documentation for the gnumi simulation software can be found at: http://www.hep.utexas. edu/ zarko/wwwgnumi.

[177] "Neutrinos from the NuMI beamline in the MiniBooNE detector", Alexis A. Aguilar-Arevalo; Prepared for Particles and Nuclei International Conference (PANIC 05), Santa Fe, New Mexico, 2428 Oct 2005. Published in AIP Conf.Proc.842:834836,200.

[178] Z. Djurcic and A. Aguilar-Arevalo, BooNE Technical Note \#238.

[179] A. Aguilar-Arevalo, MiniBooNE Technical Note \#97. 
[180] R. B. Patterson, FERMILAB-THESIS-2007-19, 251pp. Nov 2007.

[181] J. R. Monroe, FERMILAB-THESIS-2006-44, Jul 2006.

[182] M. Sorel, FERMILAB-THESIS-2005-07, UMI-31-74896, 2005.

[183] J. L. Raaf, FERMILAB-THESIS-2005-20, UMI-31-90981, 2005.

[184] D. C. Cox, Proceedings of the Fifth International Workshop on NeutrinoNucleus Interactions in the Few-GeV Region (NuInt'07), Fermilab, May 30-June $3(2007)$.

[185] C. H. Llewellyn Smith, Phys. Rept. 3C, 261 (1972).

[186] R. A. Smith and E. J. Moniz, Nucl. Phys. B43, 605 (1972), Erratum: R. A. Smith and E. J. Moniz, Nucl. Phys. B101, 547 (1975).

[187] N. J. Baker et al., Phys. Rev. D 23, 2449 (1981).

[188] V. Bernard et al., J. Phys., G28, R1 (2002).

[189] M. Gell-Mann and M. Levy, Nuovo Cimento 16, 705 (1960).

[190] A. Aguilar-Arevalo et al. [MiniBooNE Collaboration], Phys. Rev. Lett 98, $231801(2007)$.

[191] A. A. Aguilar-Arevalo et al. [MiniBooNE Collaboration], arXiv:0706.0926 [hepex]. Accepted for publication in Phys. Rev. Lett.

[192] B.P. Roe et al., Nucl. Instrum. \& Meth. A543, 577 (2005). [physics/0408124] H.J. Yang et al., Nucl. Instrum. \& Meth. A555, 370 (2005). [physics/0508045] H.J. Yang et al., Nucl. Instrum. \& Meth. A574, 342 (2007). [physics/0610276] Y. Liu et al., Nucl. Instrum. \& Meth. A578, 315 (2007). [physics/0611267] 
[193] Y. Freund, R. E. Schapire, Experiments with a new boosting algorithm, in: Proceedings of COLT, ACM Press, New York, pp. 209-217 (1996).

[194] Y. Liu, I. Stancu, Nuclear Inst., Meth., A578, 315, (2007).

[195] MINUIT, CERN program Library entry D506, available at: http://wwwasdoc.web.cern.ch/wwwasdoc/minuit/minmain.html.

[196] See the MiniBooNE algorithms group page at: http://www-boone.fnal.gov/ Main developer of the BDT input variable selection procedure: Dr. Heather Ray, University of Florida [hray@fnal.gov].

[197] L. Bartoszek, http://www. bartoszekeng.com, Bartoszek Engineering.

[198] GEANT Detector and Simulation Tool, CERN Program Library Long Writeup W5013 (1993).

[199] Hamamatsu, http://www.hamamatsu.com.

[200] B. T. Fleming, FERMILAB-THESIS-2001-02 (2001). 


\section{Appendices}




\section{Appendix A}

\section{Neutrino Energy Calibration}

\section{A.1 Selecting well reconstructed QE events}

A simple set of cuts was applied to try to isolate those QE events with a reasonably good reconstruction:

1. NUANCE Channel $=\mathrm{CCQE} \quad$ 3. $N_{\text {veto }}<6$ and $N_{\text {tank }}>100$

2. 2 Sub-Events for $\nu_{\mu}\left(1\right.$ for $\left.\nu_{e}\right) \quad$ 4. Reconstructed radius $<500 \mathrm{~cm}$

\section{A.2 Calibration Procedure}

The procedure to calculate the neutrino energy with good resolution was developed by studying the specific case of $\nu_{\mu}$ CCQE interactions. The method was seen to work reasonably well for $\nu_{e}$ CCQE interactions as well, although with slightly reduced quality. The procedure can be summarized as follows:

1. Correct the reconstructed energy of the lepton $\ell=\mu, e$, towards its generated true kinetic energy. The true lepton kinetic energy $\left(T_{\ell}\right)$ is calculated from the 
information of the generated momentum of the particle: $T_{\ell}=\sqrt{p_{\ell}^{2}+m_{\ell}^{2}}-m_{\ell}$. The correction equation is found by fitting a straight line to the profile histogram $T_{\ell}$ vs $E_{r e c}{ }^{1}$ :

\begin{tabular}{ccc}
\multicolumn{3}{c}{$T_{\ell}^{c o r r}=a T_{\ell}+b$} \\
\hline$\ell$ & $a$ & $b(\mathrm{GeV})$ \\
\hline$\mu$ & 0.8867 & 0.0927 \\
$e$ & 0.9942 & 0.0113 \\
\hline
\end{tabular}

The calibrated constants are shown in the above table for $\nu_{\mu}$ and $\nu_{e}$ CCQE interactions. The total lepton energy is calculated as $E_{\ell}^{c o r r}=T_{\ell}^{c o r r}+m_{\ell}$. where $m_{\ell}$ is the lepton mass. The comparison of the true and estimated lepton kinetic energies is shown in Figure A.1. There is a small shift from the identity line, however, the philosophy of this calibration procedure was not to fine tune the reconstructed lepton energy, but to get the best reconstructed neutrino energy.

2. Use the Quasi-Elastic formula assuming neutrons at rest to compute the neutrino energy with the corrected lepton total energy and reconstructed angle. Note that the reconstructed angle was not subject to a correction given that a very good correlation with the generated angle was observed.

$$
E_{\nu}^{Q E}=\frac{1}{2} \frac{2(M+B) E_{\ell}-\left(2 M B+B^{2}+m_{\ell}^{2}+\Delta M_{n p}^{2}\right)}{(M+B)-E_{\ell}+\sqrt{E_{\ell}^{2}-m_{\ell}^{2}} \cos \theta_{\ell}}
$$

Here $\theta_{\ell}$ is the reconstructed lepton scattering angle, $p_{\ell}=\sqrt{E_{\ell}^{2}-m_{\ell}^{2}}$ is the lepton momentum, $M=M_{p}=0.9387 \mathrm{GeV}$ is the proton mass, and $\Delta M_{n p}=$ $M_{n}^{2}-M_{p}^{2}=0.0024 \mathrm{GeV}^{2}$ is the difference in the squared masses of the neutron and proton. A binding energy of $B=-25 \mathrm{MeV}$ which differs from the value assumed in the NUANCE generator $(34 \mathrm{MeV})$ was used at the time the calibration was done, however this has a negligible effect since the smearing arising from Fermi motion of the nucleons inside the nuclei is dominant.

\footnotetext{
${ }^{1}$ In this calibration $E_{r e c}$ is the StancuFull $\mathrm{E}$ reconstructed track energy.
} 
In this step, also calculate the momentum transferred to the lepton in the interaction using the quantities calculated so far, and the formula:

$$
Q_{r e c}^{2}=2 E_{\nu}^{Q E} E_{\ell}\left(1-\beta_{\ell} \cos \theta_{\ell}\right)-m_{\ell}^{2}
$$

Here $\beta_{\ell}$ is the lepton velocity, given by $\beta_{\ell}=p_{\ell} / E_{\ell}$, and $p_{\ell}, E_{\ell}$ are obtained from the corrected muon kinetic energy of step 1.

3. Look at the profile plot of the distribution $\left(E_{\nu}^{Q E}-E_{\nu}^{G e n}\right)$ vs. $Q_{r e c}^{2}$ as calculated in the previous step. This profile can be fitted by a third degree polynomial, giving the following relation between the generated neutrino energy and reconstructed quantities $E_{\nu}^{Q E}$ and $Q^{2}$ :

$$
E_{\nu}^{G e n}=E_{\nu}^{Q E}-\left[a_{0}+a_{1}\left(Q^{2}\right)+a_{2}\left(Q^{2}\right)^{2}+a_{3}\left(Q^{2}\right)^{3}\right]
$$

In a given $Q_{r e c}^{2}$ bin, the spread of $\left(E_{\nu}^{Q E}-E_{\nu}^{G e n}\right)$ values is approximately symmetric with respect to their mean. Therefore, with this $Q_{r e c}^{2}$ dependent correction the differences $\left(E_{\nu}^{Q E}-E_{\nu}^{G e n}\right)$ become more evenly distributed around zero in all the $Q_{r e c}^{2}$ bins.

4. After making the $Q_{r e c}^{2}$ correction to $E_{\nu}^{Q E}$, the profile plot of the distribution $\left(E_{\nu}^{Q E}-E_{\nu}^{G e n}\right)$ vs. $E_{\nu}^{G e n}$ is fitted with a quadratic polynomial in the interval from 0.2 to $2.0 \mathrm{GeV}$ :

$$
\left(E_{\nu}^{Q E}-E_{\nu}^{G e n}\right)=b_{0}+b_{1}\left(E_{\nu}^{G e n}\right)+b_{2}\left(E_{\nu}^{G e n}\right)^{2}
$$

giving the following correction equation:

$$
E_{\nu}^{G e n}=\frac{\sqrt{(1+b 1)^{2}+4 b_{2}\left(E_{\nu}^{Q E}-b_{0}\right)}-(1+b 1)}{2 b_{2}}
$$


To avoid negative values in the square root, the last correction is not applied for events with $E_{\nu}^{Q E}<b_{0}$. This is a small effect, since almost all events passing the CCQE cuts result on neutrino energies grater than $200 \mathrm{MeV}$.

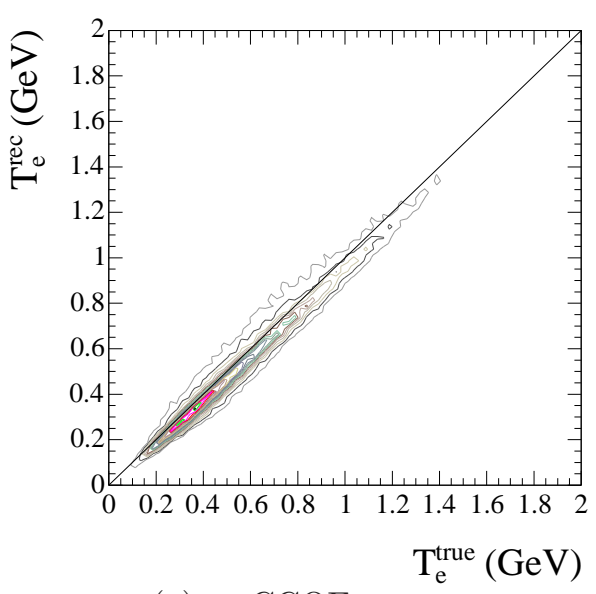

(a) $\nu_{e}$ CCQE events.

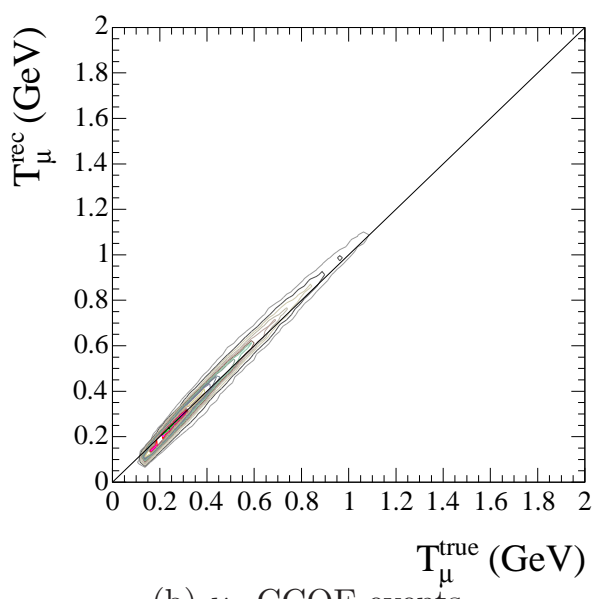

(b) $\nu_{\mu}$ CCQE events.

Figure A.1: Reconstructed vs. true kinetic energy of electrons (a), and muons (b) produced in CCQE interactions, after the corrections of step 1 of the calibration procedure. The calibration procedure aims to correct the neutrino energy and does not focus on the estimate of the lepton kinetic energy.

\section{Neutrino Energy Resolution}

The energy resolution is estimated by plotting the distribution of $E_{\nu}^{Q E}-E_{\nu}^{\text {true }} v s$. $E_{\nu}^{\text {true }}$ in $100 \mathrm{MeV}$ slices of true $E_{\nu}^{\text {true }}$, shown in Figure A.4(a) for $\nu_{\mu}$ interactions and Figure A.4(b) for $\nu_{e}$ interactions. Each resulting distribution is fit to Gaussian and the fitted widths are plotted as a function of the center of the $E_{\nu}^{\text {true interval for each }}$ slice. The Neutrino energy resolution curves so obtained are shown in Figures A.5(a) and A.5(b). 

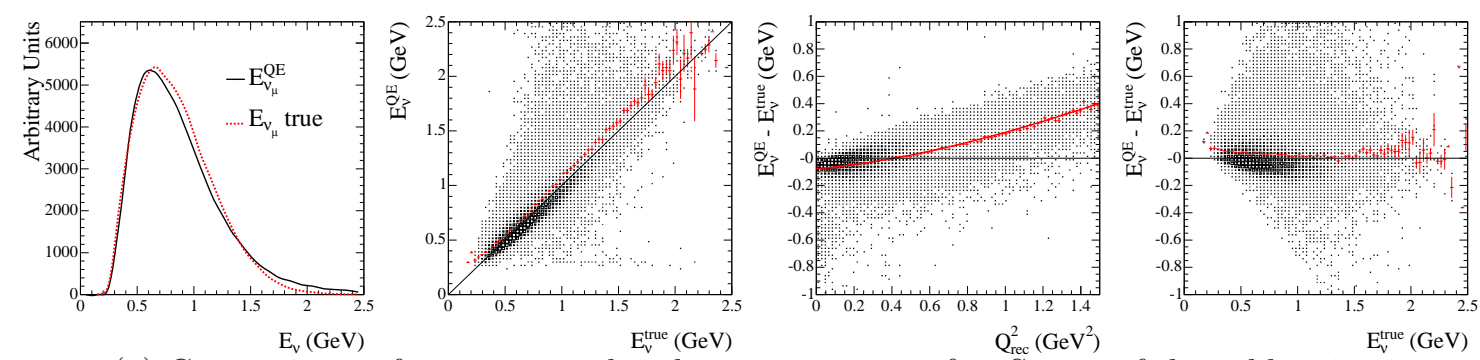

(a) Comparisons of reconstructed and true $\nu_{\mu}$ energy after Step 2 of the calibration.
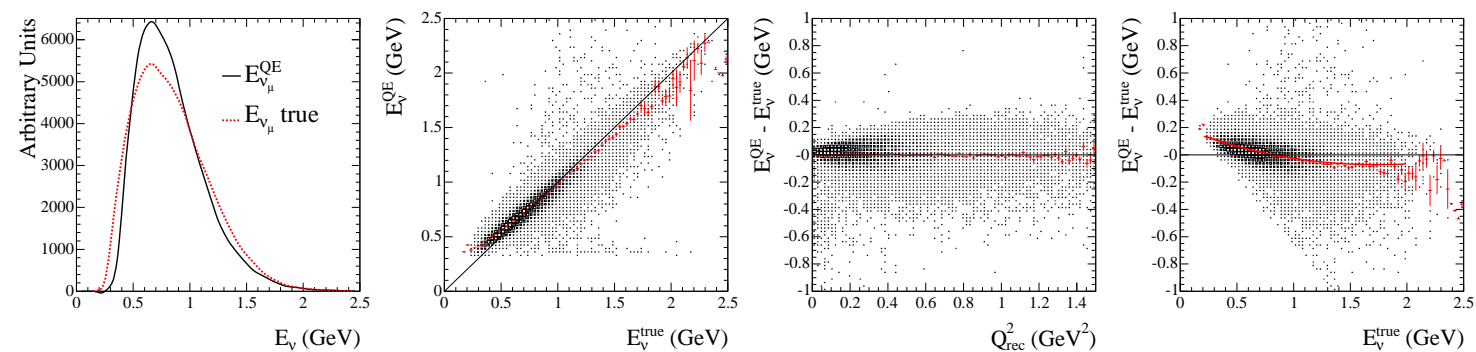

(b) Comparisons of reconstructed and true $\nu_{\mu}$ energy after Step 3 of the calibration.
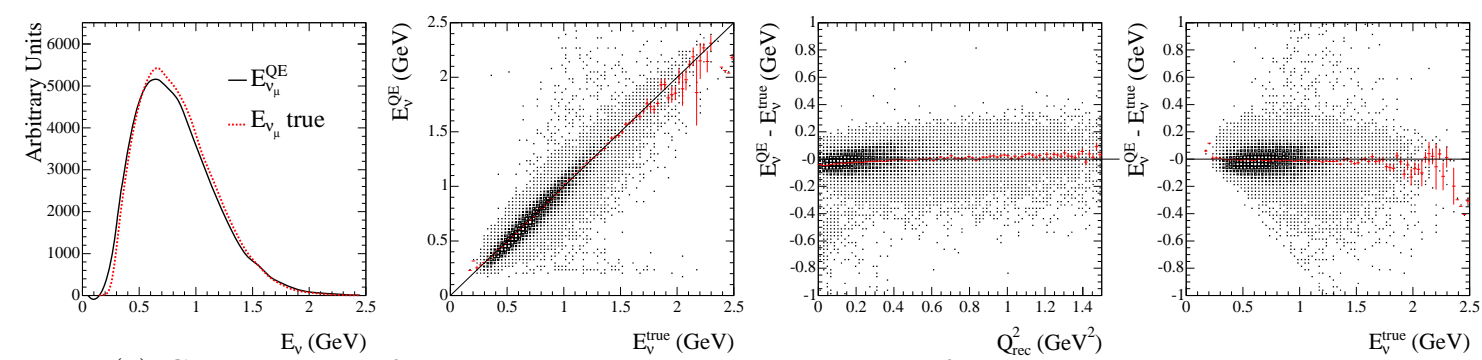

(c) Comparisons of reconstructed and true $\nu_{\mu}$ energy after all corrections are applied

Figure A.2: Reconstruction of $\nu_{\mu}$ CCQE kinematics after the second (a), third (b), and final (c) calibration steps. From left to right: Comparison of true and reconstructed $\nu_{\mu}$ energy spectra; Scatter plot and profile of reconstructed vs. true $\nu_{\mu}$ energy $E_{\nu}^{Q E}$ vs. $E_{\nu}^{\text {true }}$ ; Scatter plot and profile of residuals vs. reconstructed $Q^{2}$; Scatter plot and profile of residuals vs. true $\nu_{\mu}$ energy $E_{\nu}^{Q E}-E_{\nu}^{\text {true }}$ vs. $E_{\nu}^{\text {true }}$. 

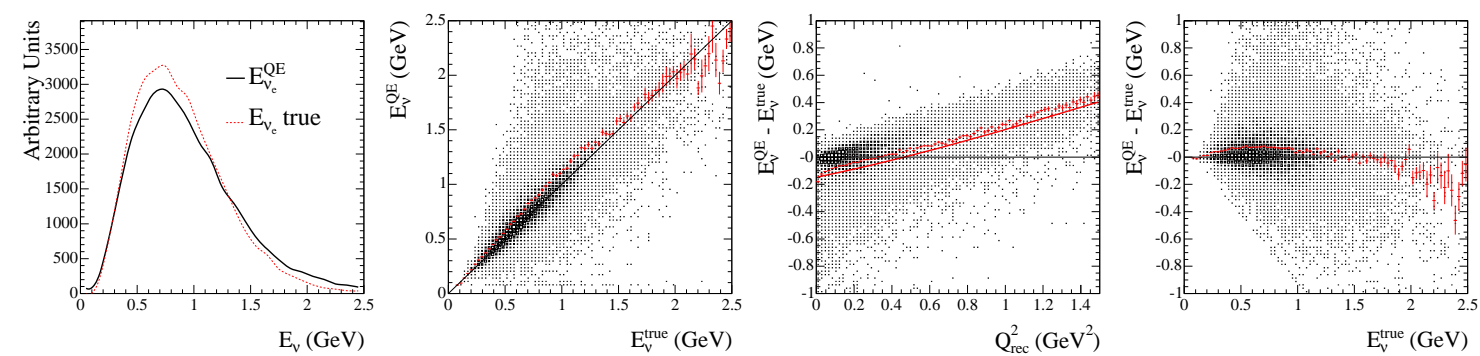

(a) Comparisons of reconstructed and true $\nu_{e}$ energy after Step 2 of the calibration.
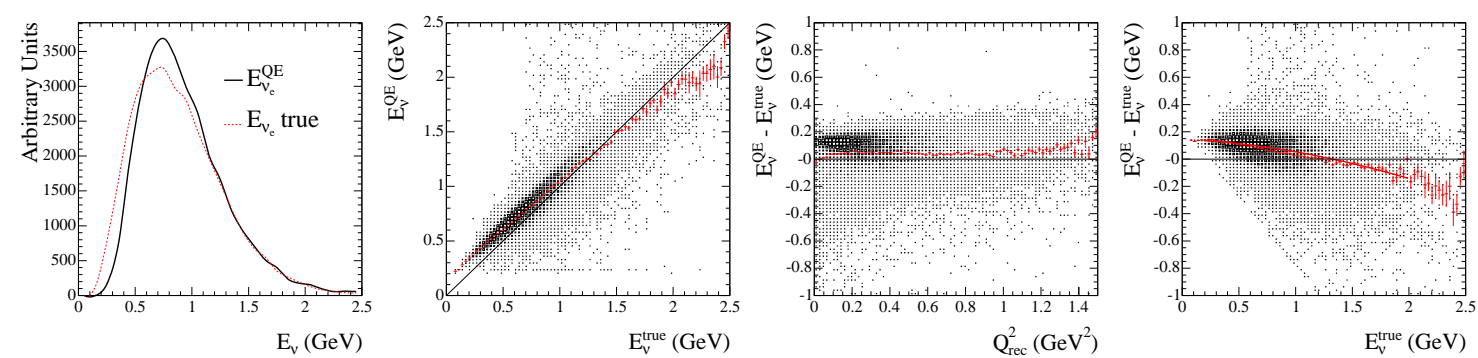

(b) Comparisons of reconstructed and true $\nu_{e}$ energy after Step 3 of the calibration.
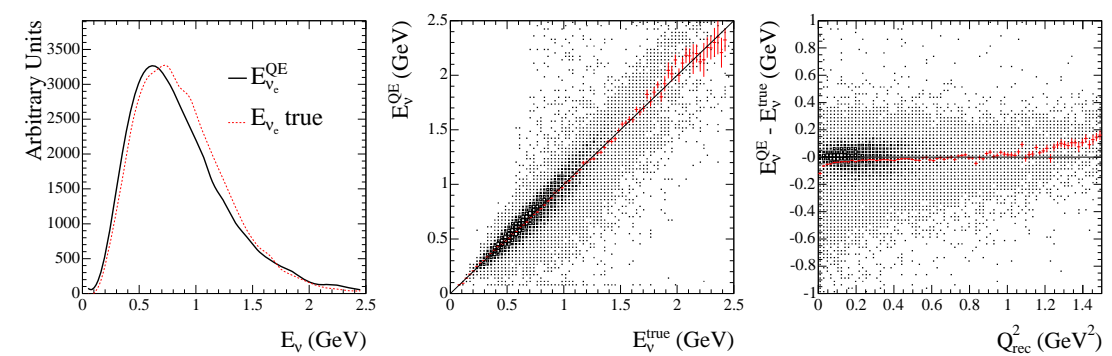

(c) Comparisons of reconstructed and true $\nu_{e}$ energy after all corrections are applied

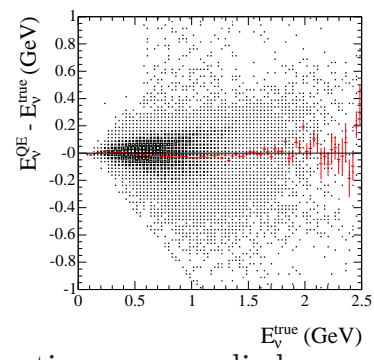

Figure A.3: Reconstruction of $\nu_{e}$ CCQE kinematics after the second (a), third (b), and final (c) calibration steps. From left to right: Comparison of true and reconstructed $\nu_{e}$ energy spectra; Scatter plot and profile of reconstructed vs. true $\nu_{e}$ energy $E_{\nu}^{Q E}$ vs. $E_{\nu}^{\text {true }}$; Scatter plot and profile of residuals vs. reconstructed $Q^{2}$; Scatter plot and profile of residuals vs. true $\nu_{e}$ energy $E_{\nu}^{Q E}-E_{\nu}^{\text {true }}$ vs. $E_{\nu}^{\text {true }}$. 

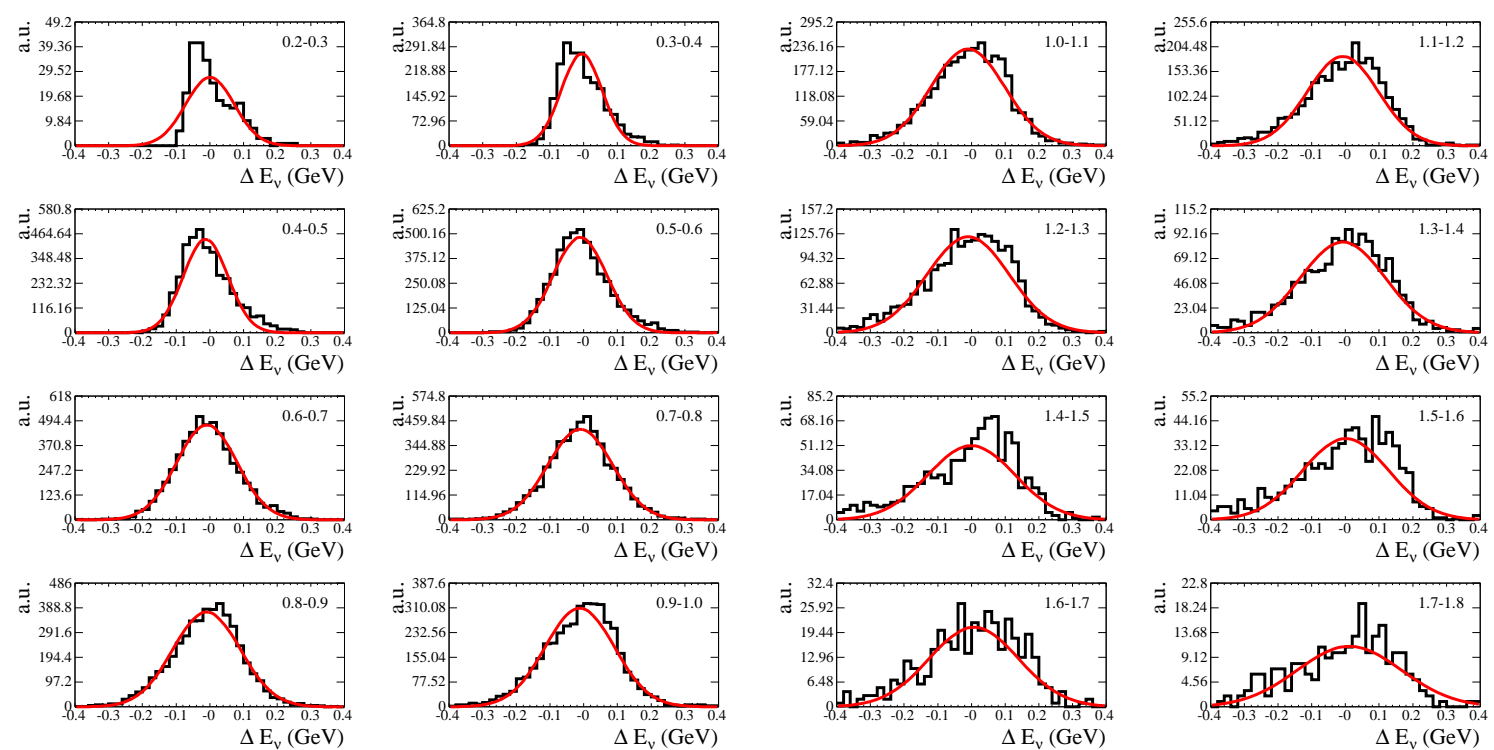

(a) $\nu_{\mu}$ CCQE events.
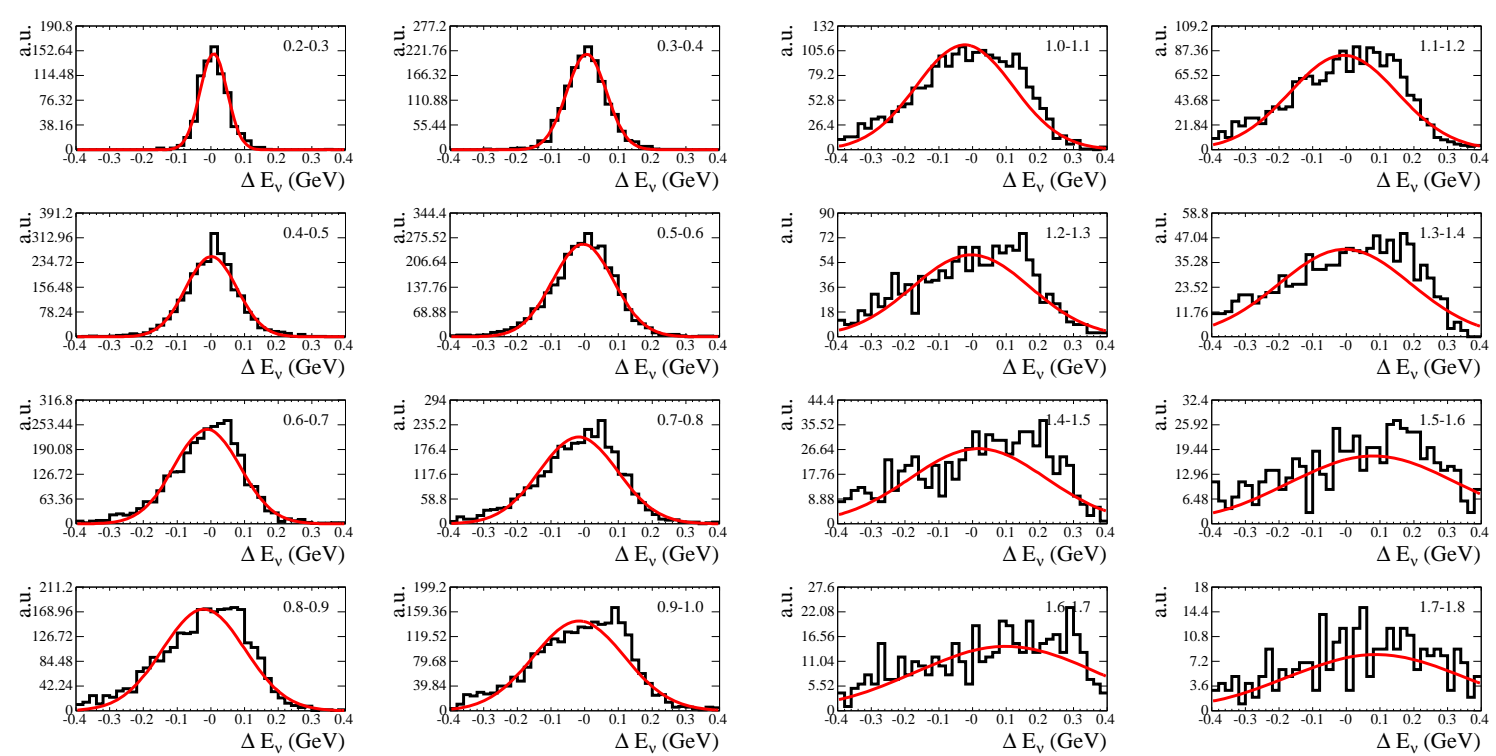

(b) $\nu_{e}$ CCQE events.

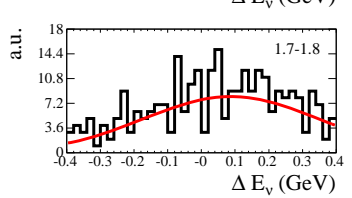

Figure A.4: Distribution of $\Delta_{E_{\nu}}$ reconstructed $E_{\nu}^{Q E}$-generated $E_{\nu}^{\text {true }}$ in $100 \mathrm{MeV}$ slices of . $E_{\nu}^{\text {true }}$.The curve is a Gaussian fit to each distribution. The true energy interval is indicated at the top-right corner of each plot in $\mathrm{GeV}$. 


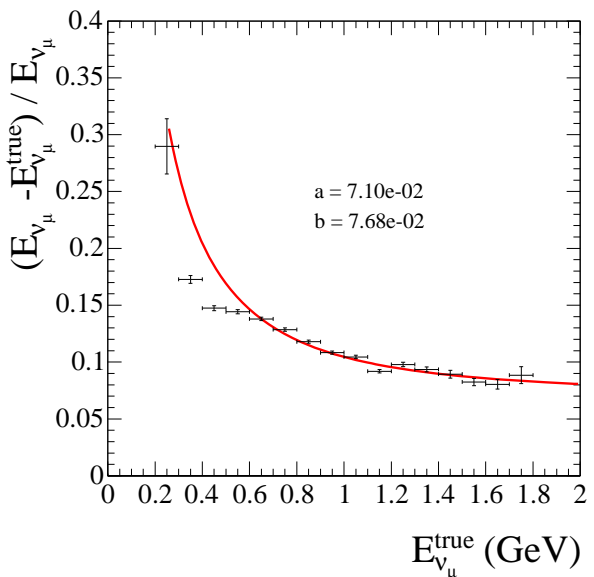

(a) $\nu_{\mu}$ CCQE events.

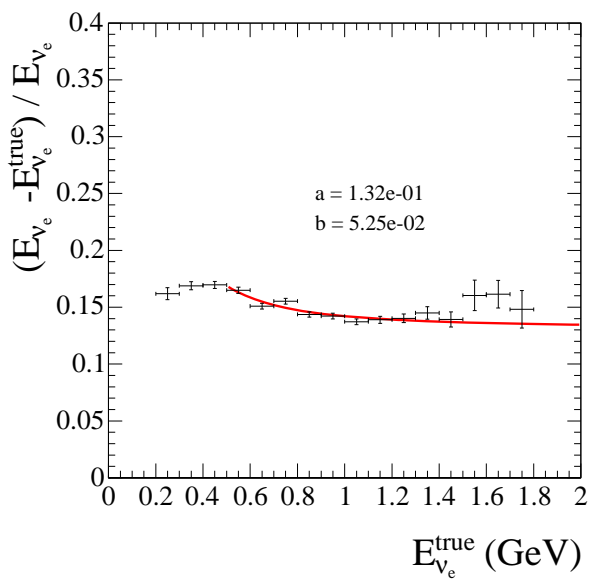

(b) $\nu_{e}$ CCQE events.

Figure A.5: Neutrino energy resolution for CCQE events as a function of generated $E_{\nu}^{\text {true }}$ in $100 \mathrm{MeV}$ bins. 


\section{Appendix B}

\section{Understanding the $\nu_{\mu}$ constraint}

\section{B.1 Example: $1-\nu_{e}$ bin $+1-\nu_{\mu}$ bin}

In order too see how the $\nu_{\mu}$ events constraint the $\nu_{e}$ events we look at the simplest possible case of a single $\nu_{e}$ bin and a single $\nu_{\mu}$ bin.

\section{Setting up the problem}

We start by defining an error matrix. If

$$
\begin{aligned}
& \sigma_{e}, \sigma_{\mu} \\
& \text { are the statistical uncertainties on the numbers of events in } \\
& \text { the } \nu_{e} \text { and } \nu_{\mu} \text { samples respectively. } \\
& \delta_{e}, \delta_{\mu} \\
& \text { are systematic uncertainties on the numbers of events in the } \\
& \nu_{e} \text { and } \nu_{\mu} \text { samples respectively. } \\
& \text { is the correlation between the } \nu_{e} \text { systematic and the } \nu_{\mu} \text { sys- } \\
& \text { tematic uncertainty. }
\end{aligned}
$$


then the error matrix for the system can be written as

$$
\mathbf{E}_{2 \times 2}=\left(\begin{array}{cc}
\sigma_{e}^{2}+\delta_{e}^{2} & \rho \delta_{e} \delta_{\mu} \\
\rho \delta_{e} \delta_{\mu} & \sigma_{\mu}^{2}+\delta_{\mu}^{2}
\end{array}\right)
$$

If we define $\alpha_{e}=\sigma_{e} / \delta_{e}$, and $\alpha_{\mu}=\sigma_{\mu} / \delta_{\mu}$ then the inverse error matrix is

$$
\mathbf{E}_{2 \times 2}^{-1}=\frac{1}{\left(\alpha_{e}^{2}+1\right)\left(\alpha_{\mu}^{2}+1\right)-\rho^{2}}\left(\begin{array}{cc}
\frac{\alpha_{\mu}^{2}+1}{\delta_{e}^{2}} & \frac{-\rho}{\delta_{e} \delta_{\mu}} \\
\frac{-\rho}{\delta_{e} \delta_{\mu}} & \frac{\alpha_{e}^{2}+1}{\delta_{\mu}^{2}}
\end{array}\right)
$$

Now turn to the $\chi^{2}$. If

$s \quad$ is the number of oscillation events in the $\nu_{e}$ sample,

$\Delta_{e}$ is the difference between the number of events in the $\nu_{e}$ sample and the number of predicted $\nu_{e}$ background events.

$\Delta_{\mu}$ is the difference between the number of events in the $\nu_{\mu}$ sample and the number of predicted $\nu_{\mu}$ events.

then the $\chi^{2}$ for the combined $\nu_{e}$ and $\nu_{\mu}$ counting experiment can be written in vector form as

$$
\chi^{2}=\left(\begin{array}{ll}
\Delta_{e}-s & \Delta_{\mu}
\end{array}\right) \mathbf{E}_{\mathbf{2} \times \mathbf{2}}^{-1}\left(\begin{array}{c}
\Delta_{e}-s \\
\Delta_{\mu}
\end{array}\right)
$$

from where

$$
\begin{aligned}
\chi^{2}= & \frac{1}{\left(\alpha_{e}^{2}+1\right)\left(\alpha_{\mu}^{2}+1\right)-\rho^{2}} \times \\
& {\left[\left(\alpha_{\mu}^{2}+1\right) \frac{\left(\Delta_{e}-s\right)^{2}}{\delta_{e}^{2}}-2 \rho \frac{\Delta_{\mu}\left(\Delta_{e}-s\right)}{\delta_{e} \delta_{\mu}}+\left(\alpha_{e}^{2}+1\right) \frac{\Delta_{\mu}^{2}}{\delta_{\mu}^{2}}\right] }
\end{aligned}
$$


Differentiating this last expression with respect to $s$ and equating to zero produces the best fit signal events:

$$
s=\Delta_{e}\left[1-\frac{\rho}{\alpha_{\mu}^{2}+1} \frac{\Delta_{\mu} / \delta_{\mu}}{\Delta_{e} / \delta_{e}}\right]
$$

and taking twice the inverse of the second derivative yields the uncertainty on the number of signal events

$$
\delta_{s}^{2}=\sigma_{e}^{2}+\left[1-\frac{\rho^{2}}{\alpha_{\mu}^{2}+1}\right] \delta_{e}^{2}
$$

1. If there is no correlation between the $\nu_{e}$ and $\nu_{\mu}$ bins $(\rho=0)$, or if the $\nu_{\mu}$ sample has large stat. uncertainty $\left(\alpha_{\mu} \longrightarrow \infty\right)$ then the extracted signal and its uncertainty are $\Delta_{e} \pm \sqrt{\left(\sigma_{e}^{2}+\delta_{e}^{2}\right)}$.

2. If the $\nu_{e}$ 's and $\nu_{\mu}$ 's are fully correlated $(\rho=1)$ and the $\nu_{\mu}$ sample has negligible statistical uncertainty $\left(\alpha_{\mu}=0\right)$, the extracted signal and its uncertainty are $\Delta_{e}\left(1-\frac{\Delta_{\mu} / \delta_{\mu}}{\Delta_{e} / \delta_{e}}\right) \pm \sigma_{e}$

Note that if we substitute $s=P N_{\mu}^{\text {pred }}$, where $P$ is an oscillation probability and $N_{\text {fullosc }}^{\text {pred }}$ is the predicted number of fullosc events, then the extracted oscillation probability has no tie to the observed $\nu_{\mu}$ data, and would be wrong. This is addressed in the following section.

\section{IMPORTANT NOTE:}

The previous derivation the elements of the error matrix $\mathbf{E}_{\mathbf{2} \times \mathbf{2}}$ are assumed independent of $s$ 


\section{Introducing the signal component error}

Start from the $3 \times 3$ matrix:

$$
\mathbf{E}_{3 \times 3}=\left(\begin{array}{ccc}
\sigma_{s}^{2}+\delta_{s}^{2} & \rho_{s b} \delta_{s} \delta_{b} & \rho_{s \mu} \delta_{s} \delta_{\mu} \\
\rho_{s b} \delta_{s} \delta_{b} & \sigma_{b}^{2}+\delta_{b}^{2} & \rho_{b \mu} \delta_{b} \delta_{\mu} \\
\rho_{s \mu} \delta_{s} \delta_{\mu} & \rho_{b \mu} \delta_{b} \delta_{\mu} & \sigma_{\mu}^{2}+\delta_{\mu}^{2}
\end{array}\right) ; \quad \mathbf{x}=\left(\begin{array}{c}
s \\
b \\
\mu
\end{array}\right)
$$

where we introduce analogous quantities to those defined earlier:

$\sigma_{s} \quad$ is the statistical uncertainty on the number of signal events $s$

$\delta_{s} \quad$ is the systematic uncertainty on the number of signal events $s$

$\delta_{b}$ is the systematic uncertainty on the number of $\nu_{e}$ background events $b$.

are the correlations between the systematic uncertainties of $\rho_{s b}, \rho_{s \mu} \quad$ the signal and $\nu_{e}$ background events, and between the signal and $\nu_{\mu}$ events. respectively.

is the correlation between the systematic uncertainties of the $\rho_{s b}$ $\nu_{e}$ and $\nu_{\mu}$ events.

\section{Error matrix propagation}

Propagation of this covariance $\left(\mathbf{E}_{3 \times 3}=\operatorname{cov}\left(x_{l}, x_{m}\right)\right)$ matrix through the linear transformation $\mathbf{f}(\mathbf{x})$ whose Jacobian matrix is show below:

$$
\mathbf{f}(\mathbf{x})=\left(\begin{array}{c}
s+b \\
\mu \\
0
\end{array}\right), \quad \mathcal{J}=\left(\begin{array}{ccc}
1 & 1 & 0 \\
0 & 0 & 1 \\
0 & 0 & 0
\end{array}\right)
$$


will be accomplished by:

$$
\operatorname{cov}\left(f^{i}, f^{j}\right)=\sum_{l, m}^{\operatorname{Dim}(\mathbf{x})}\left(\frac{\partial f^{i}}{\partial x_{l}}\right)\left(\frac{\partial f^{j}}{\partial x_{m}}\right) \operatorname{cov}\left(x_{l}, x_{m}\right)
$$

Or simply:

$$
\mathbf{E}_{3 \times 3}^{\prime}=\mathcal{J} \times \mathbf{E}_{3 \times 3} \times \mathcal{J}^{\mathrm{T}}
$$

The $2 \times 2$ matrix defined by the non-zero elements of $\mathbf{E}_{3 \times 3}^{\prime}$ is our covariance matrix in $e$ and $\mu$ bins that includes the correlations between $s$ and $b$ :

$$
\mathbf{E}_{2 \times 2}=\left(\begin{array}{ccc|c}
\sigma_{s}^{2}+\delta_{s}^{2} & & \rho_{s b} \delta_{s} \delta_{b} & \rho_{s \mu} \delta_{s} \delta_{\mu} \\
& + & & + \\
\rho_{s b} \delta_{s} \delta_{b} & & \sigma_{s}^{2}+\delta_{s}^{2} & \rho_{b \mu} \delta_{b} \delta_{\mu} \\
\hline & & \\
\rho_{s \mu} \delta_{s} \delta_{\mu} & +\rho_{b \mu} \delta_{b} \delta_{\mu} & \sigma_{\mu}^{2}+\delta_{\mu}^{2}
\end{array}\right) ; \quad \mathbf{x}=\left(\begin{array}{c}
s+b \\
\mu
\end{array}\right)
$$

Making the following definitions:

$$
\begin{aligned}
\sigma_{e}^{2} & =\sigma_{s}^{2}+\sigma_{b}^{2} \\
\delta_{e}^{2} & =\delta_{s}^{2}+\delta_{b}^{2}+2 \rho_{s b} \delta_{s} \delta_{b} \\
\rho & =\left(\rho_{s \mu} \delta_{s}+\rho_{b \mu} \delta_{b}\right) / \delta_{e}
\end{aligned}
$$

we write the matrix $\mathbf{E}_{2 \times 2}$ in the notation of the first section:

$$
\mathbf{E}_{2 \times 2}=\left(\begin{array}{cc}
\sigma_{e}^{2}+\delta_{e}^{2} & \rho \delta_{e} \delta_{\mu} \\
\rho \delta_{e} \delta_{\mu} & \sigma_{\mu}^{2}+\delta_{\mu}^{2}
\end{array}\right)
$$

Solving first the problem where there is no signal contribution to the error matrix $\mathbf{E}_{2 \times 2}$ arriving at expressions (B.1), and (B.1) reproduced here:

$$
\begin{gathered}
s=\Delta_{e}\left[1-\frac{\rho}{\alpha_{\mu}^{2}+1} \frac{\Delta_{\mu} / \delta_{\mu}}{\Delta_{e} / \delta_{e}}\right] \\
\delta_{s}^{2}=\sigma_{e}^{2}+\left[1-\frac{\rho^{2}}{\alpha_{\mu}^{2}+1}\right] \delta_{e}^{2}
\end{gathered}
$$




\section{Iterative approach}

We now allow the following quantities to be functions of $s$ :

$$
\begin{aligned}
\sigma_{e} & =\sigma_{e}(s) \\
\delta_{e} & =\delta_{e}(s) \\
\rho & =\rho(s)
\end{aligned}
$$

but neglect the dependence of the covariance matrix on $s$ to minimize the $\chi^{2}$ at each step. We then recalculate the matrix using the previously obtained best value of $s$, and minimize again to find the next iteration of $s$.

In this scheme, the value of $s$ that minimizes $\chi^{2}$ at iteration $n+1$ is given in terms of the matrix evaluated at iteration $n$ by:

$$
s^{(n+1)}=\Delta_{e}\left[1-\frac{\rho^{(n)}}{\alpha_{\mu}^{2}+1} \frac{\Delta_{\mu} / \delta_{\mu}}{\Delta_{e} / \delta_{e}^{(n)}}\right]
$$

and the error on the signal is:

$$
\left(\delta_{s}^{(n+1)}\right)^{2}=\left(\sigma_{e}^{(n)}\right)^{2}+\left[1-\frac{\left(\rho^{(n)}\right)^{2}}{\alpha_{\mu}^{2}+1}\right]\left(\delta_{e}^{(n)}\right)^{2}
$$

\section{Iterative solution for the signal:}

We first rewrite $s^{(n+1)}$ as follows:

$$
s^{(n+1)}=\Delta_{e}-\frac{\Delta_{\mu}}{\delta_{\mu}\left(\alpha_{\mu}^{2}+1\right)} \rho^{(n)} \delta_{e}^{(n)} .
$$

Using the definition in Eq.(B.12), the combination $\rho^{(n)} \delta_{e}^{(n)}$ is:

$$
\rho^{(n)} \delta_{e}^{(n)}=\rho_{s \mu}^{(n)} \delta_{s}^{(n)}+\rho_{b \mu} \delta_{b}
$$

We now write $\delta_{s}^{(n)}=\delta_{s}^{\text {frac }} \times s$, where $\delta_{s}^{\text {frac }}$ is the fractional systematic error on $s$, which is the same in all iterations. Note that also the correlation $\rho_{s \mu}^{(n)}$ between $s$ and 
$\mu$ is the same in all iterations, so we will drop the iteration index from it, and write:

$$
s^{(n+1)}=\Delta_{e}-\frac{\Delta_{\mu}}{\delta_{\mu}\left(\alpha_{\mu}^{2}+1\right)}\left[\rho_{s \mu} \delta_{s}^{f r a c} \times s^{(n)}+\rho_{b \mu} \delta_{b}\right]
$$

Defining the following constants:

$$
\begin{aligned}
A & =-\frac{\Delta_{\mu}}{\delta_{\mu}\left(\alpha_{\mu}^{2}+1\right)} \rho_{s \mu} \delta_{s}^{\text {frac }} \\
B & =\Delta_{e}-\frac{\Delta_{\mu}}{\delta_{\mu}\left(\alpha_{\mu}^{2}+1\right)} \rho_{b \mu} \delta_{b},
\end{aligned}
$$

we see that the recursion relation is simply expressed as:

$$
s^{(n+1)}=A s^{(n)}+B
$$

If we use the index $n=0$ to denote the starting values (for example $s^{(0)}=0$ ), we find that the recursion relation implies:

$$
s^{(n)}=(A)^{n} s^{(0)}+B \sum_{l=1}^{n}(A)^{l} .
$$

If $|A|<1$ the sequence will converge when $n \rightarrow \infty$ by the vanishing of the first term and the convergence of the geometric series in the second term:

$$
s^{(\infty)}=\frac{B}{1-A}
$$

or

$$
s=\frac{1}{\left(1+\frac{\Delta_{\mu}}{\delta_{\mu}\left(\alpha_{\mu}^{2}+1\right)} \rho_{s \mu} \delta_{s}^{\text {frac }}\right)}\left[\Delta_{e}-\frac{\Delta_{\mu}}{\delta_{\mu}\left(\alpha_{\mu}^{2}+1\right)} \rho_{b \mu} \delta_{b}\right]
$$

\section{Convergence of the iterative approach}

$|A|>1$ would require that the difference between the observed an predicted $\nu_{\mu}$ 's be larger than the number of predicted events. Such a fluctuation should be very rare. 


\section{Extracting an oscillation probability}

Rearranging the terms we write:

$$
s=\frac{1}{\left(1+\frac{\Delta_{\mu}}{\delta_{\mu}\left(\alpha_{\mu}^{2}+1\right)} \rho_{s \mu} \delta_{s}^{\text {frac }}\right)} \Delta_{e}\left[1-\frac{\rho_{b \mu}}{\alpha_{\mu}^{2}+1} \frac{\Delta_{\mu} / \delta_{\mu}}{\Delta_{e} / \delta_{b}}\right]
$$

If the signal $s$ is due to an oscillation, we can extract the oscillation probability by writing:

$$
s=P N_{\mu}^{\text {fullosc }} \quad P=P\left(\sin ^{2} 2 \theta, \Delta m^{2}\right)
$$

The quantity $P$ is the energy-averaged oscillation probability, and $N_{\mu}^{\text {fullosc }}$ is the predicted number of fully oscillated $\nu_{\mu}$ events that interacted as $\nu_{e}$ 's in the detector.

The extracted value of this oscillation probability is given by:

$$
P=\frac{1}{N_{\mu}^{\text {fullosc }}\left(1+\frac{\Delta_{\mu}}{\delta_{\mu}\left(\alpha_{\mu}^{2}+1\right)} \rho_{s \mu} \delta_{s}^{\text {frac }}\right)} \Delta_{e}\left[1-\frac{\rho_{b \mu}}{\alpha_{\mu}^{2}+1} \frac{\Delta_{\mu} / \delta_{\mu}}{\Delta_{e} / \delta_{b}}\right]
$$

Here we note that the factor in the denominator, which we will denote $f_{s \mu}$ :

$$
f_{s \mu}=N_{\mu}^{\text {fullosc }}\left(1+\frac{\Delta_{\mu}}{\delta_{\mu}\left(\alpha_{\mu}^{2}+1\right)} \rho_{s \mu} \delta_{s}^{\text {frac }}\right)
$$

represents a corrected number of fully oscillated $\nu_{\mu}$ 's interacting as $\nu_{e}$ 's.

It is easy to see that in the case when $s$ and $\mu$ are $100 \%$ correlated and the statistical error in $\mu$ is negligible (i.e. $\alpha_{\mu} \approx 0$ ), the correction factor is simply:

$$
f_{s \mu} \approx N_{\mu}^{\text {fullosc }} \times \frac{N_{\mu}^{\text {obs }}}{N_{\mu}^{\text {Pred }}}
$$


where we have taken $\Delta_{\mu}=N_{\mu}^{\text {obs }}-N_{\mu}^{\text {pred }}, \delta_{\mu}=\delta_{\mu}^{\text {frac }} N_{\mu}^{\text {Pred }}$, and have assumed that the fractional systematic errors satisfy $\delta_{\mu}^{\text {frac }} \approx \delta_{s}^{\text {frac }}$.

Therefore, the extraction of an oscillation probability with the combined sample has made use of the observed $\nu_{\mu}$ spectrum to correct the predicted number of fullosc events.

\section{Conclusion}

- The constraint from the $\nu_{\mu}$ 's on the systematic errors in the $\nu_{e}$ 's is clearly identified with the presence of correlations between the $\nu_{\mu}$ and $\nu_{e}$ uncertainties.

- When the signal component of the error matrix is introduced along with the correlations that it has with the $\nu_{\mu}$ sample, an automatic normalization correction is applied to the predicted fullosc events which we use to extract an oscillation probability from an excess of events.

- This simple calculation gives important insight into the behavior of the Combined oscillations fit. 


\section{Appendix C}

\section{A frequentist study with the BDT analysis.}

\section{C.1 Introduction}

The CombinedFit ${ }^{1}$ package produces as part of its output a plot of the confidence level intervals in the space of oscillation parameters $\Delta m^{2}$ and $\sin ^{2} 2 \theta$. In the past these contours have been calculated as constant level slices of the $\chi^{2}$ surface obtained by comparing the predicted distributions for different points of the oscillations parameter space with the distribution for a given data set, using the error matrix with signal component equal to the best fit value to the data. The levels of the slices have been taken to be the textbook values for a two degree of freedom (d.o.f.) fit.

Here we describe the method to obtain such regions based in a modification of the frequentist approach explained in the paper of 1998 by Feldman and Cousins [169].

\footnotetext{
${ }^{1}$ http://www-boone.fnal.gov/software_and_analysis/current_framework/doc/index.html.
} This study can also be found in Ref.[174] and its addenda. 


\section{C.2 Finding the best fit oscillation parameters}

Given a data set with an observed $E_{\nu}^{Q E}$ distribution for $\nu_{\mu}$ candidates and one for $\nu_{e}$ candidates, we compare them with corresponding Monte Carlo distributions for various signals across the oscillations parameter space and find the parameter set that minimizes a $\chi^{2}$ statistic of the form

$$
\chi^{2}=\sum_{i, j}\left[N_{i}^{\text {data }}-N_{i}^{M C}\left(\Delta m^{2}, \sin ^{2} 2 \theta\right)\right] M_{i j}^{-1}\left[N_{j}^{\text {data }}-N_{j}^{M C}\left(\Delta m^{2}, \sin ^{2} 2 \theta\right)\right]
$$

where the matrix $M_{i j}$ is the covariance matrix in bins of $E_{\nu}^{Q E}$. Notice that in fact the covariance matrix depends on the oscillations parameters $\left(\Delta m^{2}, \sin ^{2} 2 \theta\right)$ and therefore the straightforward minimization of this $\chi^{2}$ may yield undesired results, for it is a well known effect in this kind of procedure that one way to obtain a very small value of $\chi^{2}$ is to make the inverse matrix $M_{i j}^{-1}$ go to zero, no matter how large the difference between the data and Monte Carlo distributions are.

In this type of situation, a better method to find the best fit oscillations parameters is an iterative scheme in which the error matrix is first calculated assuming there is no signal and is kept constant while varying the oscillations parameters in the differences $\Delta_{i}\left(N_{i}^{\text {data }}-N_{i}^{M C}\right)$. Once the minimum has been found using the matrix with no signal, the matrix is recalculated with the parameters found to minimize the $\chi^{2}$ the first time. A new minimization is then performed, again, keeping the new matrix fixed throughout. This procedure is repeated until a desired convergence criterion is met. Typical number of iterations range from the mode of 2 iterations, to a number less than 6 in very extreme cases.

The result of such a procedure is a set of best fit parameters that correspond to the minimum of the $\chi^{2}$ surface defined between the data and all possible predictions, when the covariance matrix is fixed at the best fit parameters themselves. 


\section{C.3 Ordering Principle}

In order to determine confidence regions in the parameter space one can follow the procedure depicted in Figure C.1.

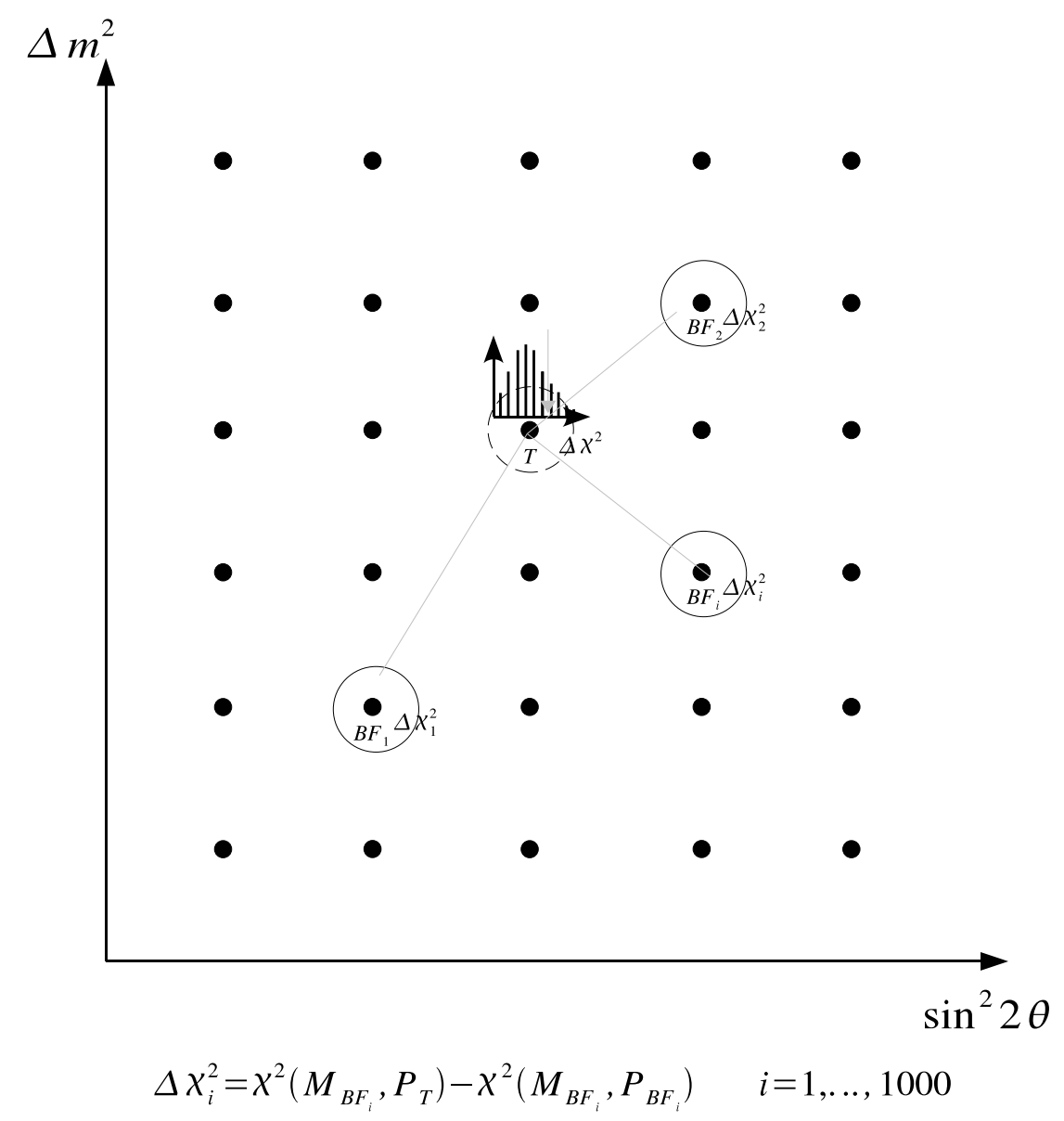

Figure C.1: Region ordering principle.

We pick one point in the parameter space and generate, say, 1000 fake data sets, 
all having the same true signal corresponding to the chosen point ( $T$ in Figure C.1). The data sets are fluctuated versions of the Monte Carlo prediction drawn from the error matrix at point $T$ in the parameter space.

Each of the 1000 data sets is run through the fitting code and we obtain for each one a best fit point (labeled $B F_{i}$ in Figure C.1), which may in general be different from the true signal point. At the same time we calculate for each of the data sets the $\chi^{2}$ value using the matrix at the best fit point, but evaluating the Monte Carlo predicted signal with the true parameters, and take the difference:

$$
\Delta \chi_{i}^{2}=\chi^{2}\left(M_{B F_{i}}, P_{T}\right)-\chi^{2}\left(M_{B F_{i}}, P_{B F_{i}}\right), \quad i=1, \ldots, 1000
$$

where $P_{T}$ represents that the signal distribution is calculated with the true signal parameters, and $P_{B F_{i}}$ represents that the signal distribution is calculated with the the best fit parameters obtained for the $i^{\text {th }}$ fake data set.

We now form the distribution of $\Delta \chi_{i}^{2}$ values for the $i=1, \ldots, 1000$ fake experiments and find for this true signal point a value $\Delta \chi_{c}^{2}(T)$ that satisfies

$$
\operatorname{Prob}\left(\Delta \chi^{2}<\Delta \chi_{c}^{2}(T)\right)=0.9
$$

to find, say, the $90 \%$ C.L. region. This cut value in the distribution of $\Delta \chi_{i}^{2}$ values tells us that if the true parameters are given by the point $P_{T}$, there is a $90 \%$ probability to find a best fit point $P_{B F_{i}}$ for which the value $\Delta \chi_{i}^{2}$ in Eq.(C.2) is smaller than $\Delta \chi_{c}^{2}(T)$.

Moreover, since the $\chi^{2}$ values are all calculated using the matrix at the best fit point for each experiment, the $\Delta \chi_{i}^{2}$ values represent likelihood ratios measuring how likely it is that the true parameter lies a certain distance away from the best fit point that was found.

Proceeding in this way for all the points in a grid of the parameter space we will form a surface of $\Delta \chi_{c}^{2}$ cut values representing the likelihood that the best fit will be found with a certain confidence (eg. $90 \%$ ), at a given distance away from each point. 
To form the desired confidence region in the parameter space we now turn to the actual data and find the best fit point for it, which we will call $P_{B F_{D}}$, and calculate, for all points $P$ in the space the surface:

$$
\chi^{2}\left(M_{B F_{D}}, P\right)=\sum_{i, j}\left[N_{i}^{d a t a}-N_{i}^{M C}(P)\right] M_{i j}^{-1}\left[N_{j}^{d a t a}-N_{j}^{M C}(P)\right]
$$

where it is understood that the error matrix is calculated at the best fit point, while the differences $\left[N_{j}^{\text {data }}-N_{j}^{M C}(P)\right]$ vary from point to point in the space. This surface contains the information about comparing the data distribution with all the points of the parameter space, while assuming that the error matrix is given by the best fit parameters.

Note that the data distribution can be thought of as just another one of the fake experiments. However, it is the only tie to reality that the experiment will have. Therefore, using the surface so defined seems appropriate.

We will define the acceptance region for the confidence level that gave us the cut values $\Delta \chi_{c}^{2}$ before, as the collection of points $P$ on the parameter space satisfying

$$
\chi^{2}\left(M_{B F_{D}}, P\right)-\chi^{2}\left(M_{B F_{D}}, P_{B F_{D}}\right)<\Delta \chi_{c}^{2}(P)
$$

this is, the intersection of the surface of cut values $\Delta \chi_{c}^{2}(P)$ with the surface

$$
\Delta \chi^{2}\left(M_{B F_{D}}, P\right)=\chi^{2}\left(M_{B F_{D}}, P\right)-\chi^{2}\left(M_{B F_{D}}, P_{B F_{D}}\right)
$$

\section{C.4 Alternative possibilities}

One may define the ordering principle in four different ways similar to Eq.(C.2):

A) $\quad \Delta \chi_{i}^{2}=\chi^{2}\left(M_{T}, P_{T}\right)-\chi^{2}\left(M_{T}, P_{B F_{i}}\right), \quad i=1, \ldots, 1000$ 


$$
\begin{gathered}
\Delta \chi_{i}^{2}=\chi^{2}\left(M_{T}, P_{T}\right)-\chi^{2}\left(M_{B F_{i}}, P_{B F_{i}}\right), \quad i=1, \ldots, 1000 \\
\text { C) } \Delta \chi_{i}^{2}=\chi^{2}\left(M_{B F_{i}}, P_{T}\right)-\chi^{2}\left(M_{T}, P_{B F_{i}}\right), \quad i=1, \ldots, 1000 \\
\text { D) } \Delta \chi_{i}^{2}=\chi^{2}\left(M_{B F_{i}}, P_{T}\right)-\chi^{2}\left(M_{B F_{i}}, P_{B F_{i}}\right), \quad i=1, \ldots, 1000
\end{gathered}
$$

We recognize $D$ ) as the method we discussed earlier. What distinguishes these 4 possibilities is which matrix is being used to calculate the $\chi^{2}$ values at the true point versus the best fit point.

Given our iterative minimization procedure, we immediately deem methods $A$ ) and $C$ ) as impractical because the best fit point is not guaranteed to be a minimum over a $\chi^{2}$ surface that has not been calculated using the matrix at the best fit point, allowing for negative values of $\Delta \chi_{i}^{2}$.

With respect to method $B$ ), it is clear that it will give non negative values of $\Delta \chi_{i}^{2}$ when $P_{T}$ is a null signal point, for by construction of the minimization algorithm one has that

$$
\chi^{2}\left(M_{B F_{i}}, P_{B F_{i}}\right) \leq \chi^{2}\left(M_{\text {null }}, P_{\text {null }}\right)
$$

However, it is not guaranteed that this will be true for all points in the space. Therefore, we must choose method $D$, which is guaranteed to yield a positive value of the ordering statistic $\Delta \chi_{i}^{2}$ by the iterative minimization method.

\section{C.5 Example with a $6 \times 6$ grid}

A test calculation was run defining a $6 \times 6$ grid in the parameter space and running 300 fake experiments for each of the 36 chosen points. Figure C.3 (Figure C.5) shows the distribution of $\Delta \chi_{i}^{2}$ values from which the cut $\Delta \chi_{c}^{2}$ that defines the $90 \%$ C.L. limit for each point in the grid is extracted. In this figure only statistical errors (all 
errors $)^{2}$ are included. The surfaces defined by these cut values are shown in the top plot of Figure C.4 (Figure C.6). The implementation of the method to actually find the contour by applying the criterion in Eq.(C.5) is shown in the bottom plot of Figure C.4 (Figure C.6) for a data set with background identical to the prediction and no signal.

It is clear that a good determination of these contours will require a larger number of experiments than the 300 used for the purposes of this study. A much finer grid is also desirable.

In [169] the authors report that one "... might naively expect that $\Delta \chi_{c}^{2}=4.61$, the $90 \%$ C.L. value for a $\chi^{2}$ distribution with two degrees of freedom...". Their model used statistical errors only and they found that "...it actually varies from about 2.4 to 6.6 across the $\sin ^{2}(2 \theta)-\Delta m^{2}$ plane. "It is interesting to compare these numbers with the $\Delta \chi_{c}^{2}$ values found for each distribution in Figure C.3 which range from 3.15 to $6.45^{3}$.

Table C.1: $\chi^{2}$ values that define the various central confidence intervals for different numbers of dof [171].

\begin{tabular}{|c|c|c|c|c|c|c|c|c|}
\hline Interval & Prob & 1 & 2 & 3 & 4 & 5 & 6 & $\leftarrow d o f$ \\
\hline $1 \sigma$ & 0.3173 & 1.00 & 2.30 & 3.53 & 4.72 & 5.89 & 7.04 & \\
\hline $2 \sigma$ & $0.4550 \mathrm{E}-01$ & 4.00 & 6.18 & 8.03 & 9.72 & 11.31 & 12.85 & \\
\hline $3 \sigma$ & $0.2700 \mathrm{E}-02$ & 9.00 & 11.83 & 14.15 & 16.25 & 18.20 & 20.06 & \\
\hline $4 \sigma$ & $0.6300 \mathrm{E}-04$ & 16.01 & 19.34 & 22.06 & 24.51 & 26.78 & 28.92 & \\
\hline $5 \sigma$ & $0.5700 \mathrm{E}-06$ & 24.92 & 28.67 & 31.15 & 34.20 & 26.88 & 39.33 & \\
\hline 90\%C.L. & 0.1000 & 2.71 & 4.61 & 6.25 & 7.78 & 9.24 & 10.64 & \\
\hline
\end{tabular}

${ }^{2}$ For this calculation the overestimated errors labeled elbg-uz-smoothed were used.

${ }^{3}$ Studies done with other selection cuts suggest that adding more statistics will take the values near the NULL signal point closer to the 1 dof value of 2.71 . 
When the systematic errors are introduced, regions in which $\sin ^{2}(2 \theta)$ and $\Delta m^{2}$ are strongly correlated, and therefore used to behave as having only $\sim 1$ effective dof in the statistics only case, will begin to act more as having a larger effective number of $d o f$. An example of this behavior can be seen in Figure C.2

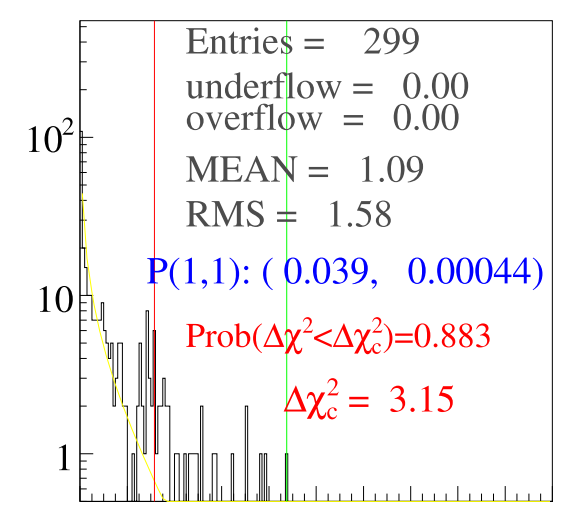

$\begin{array}{llllllll}0 & 2 & 4 & 6 & 8 & 101214161820\end{array}$

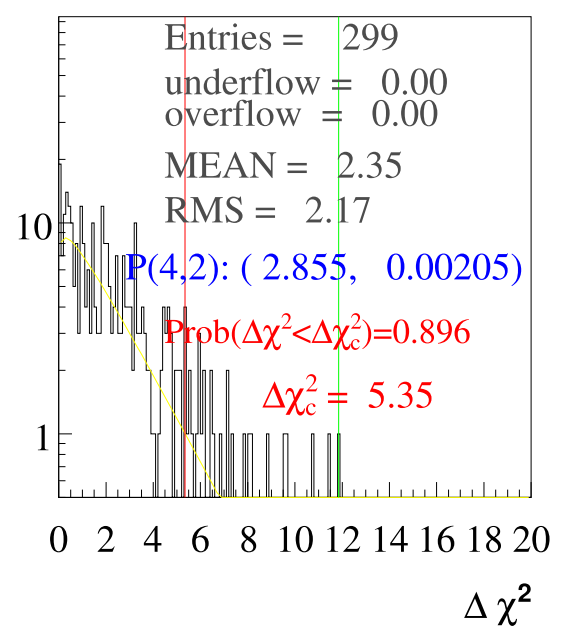

(b) Statistical errors, grid point $\left(\Delta m^{2}, \sin ^{2} 2 \theta\right)=(2.855,0.00205)$

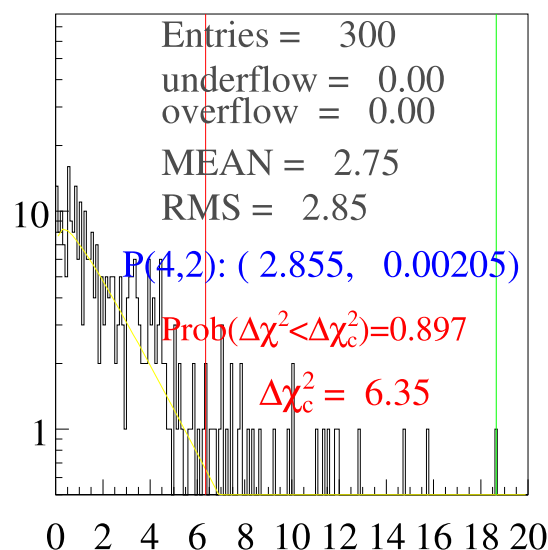

$\Delta \chi^{2}$

(c) Stat+Systematic errors, grid point (d) Stat+Systematic errors, grid point $\left(\Delta m^{2}, \sin ^{2} 2 \theta\right)=(0.039,0.00044) \quad\left(\Delta m^{2}, \sin ^{2} 2 \theta\right)=(2.855,0.00205)$

Figure C.2: $\Delta \chi_{c}^{2}$ distributions for two points in the grid.

For comparison, Table C.1 lists the values expected for central confidence intervals 
for various numbers of $d o f$.
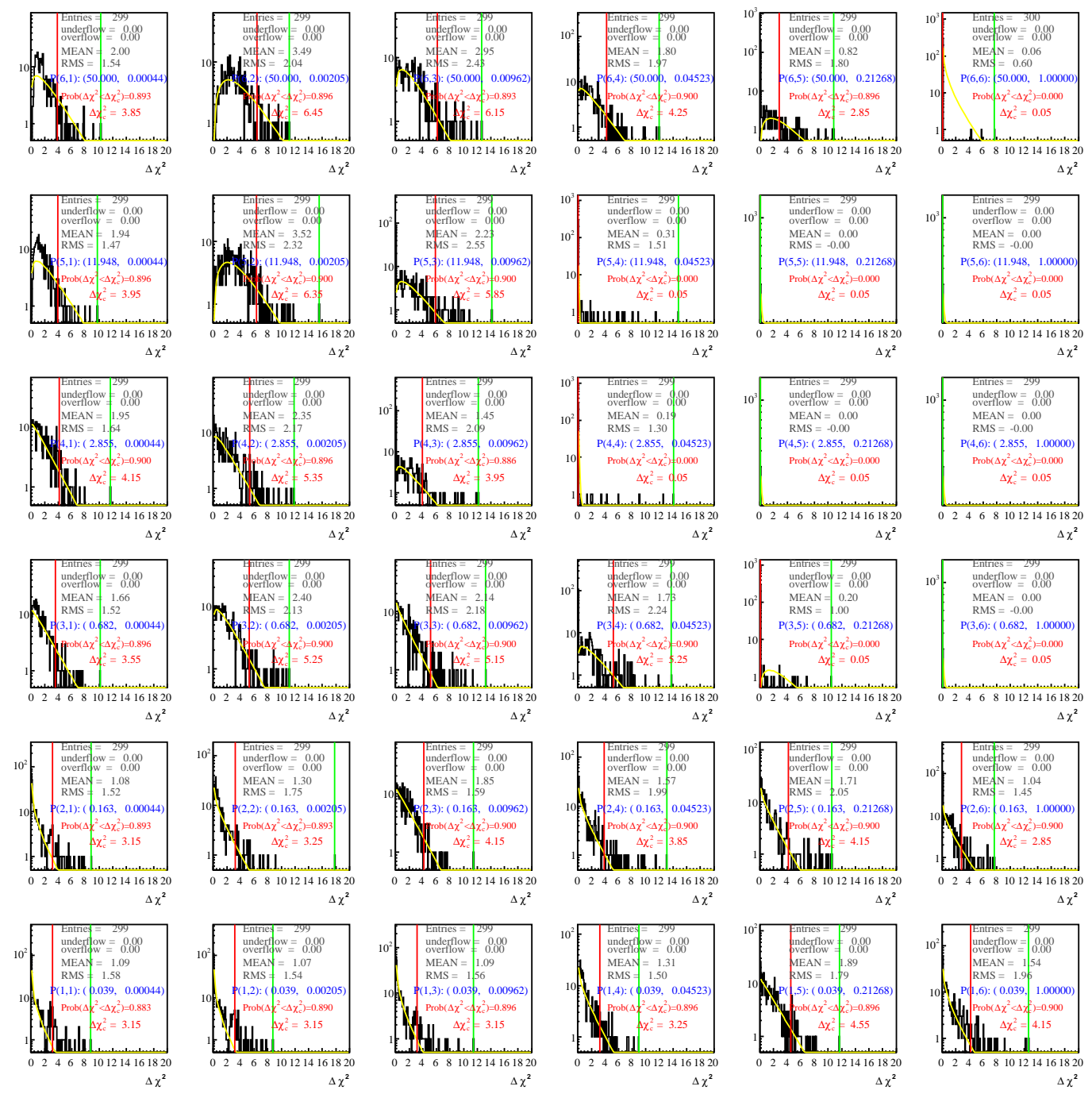

Figure C.3: Statistical errors only: $\Delta \chi^{2}$ distributions for a grid of $6 \times 6$ points in the oscillations parameter space. 


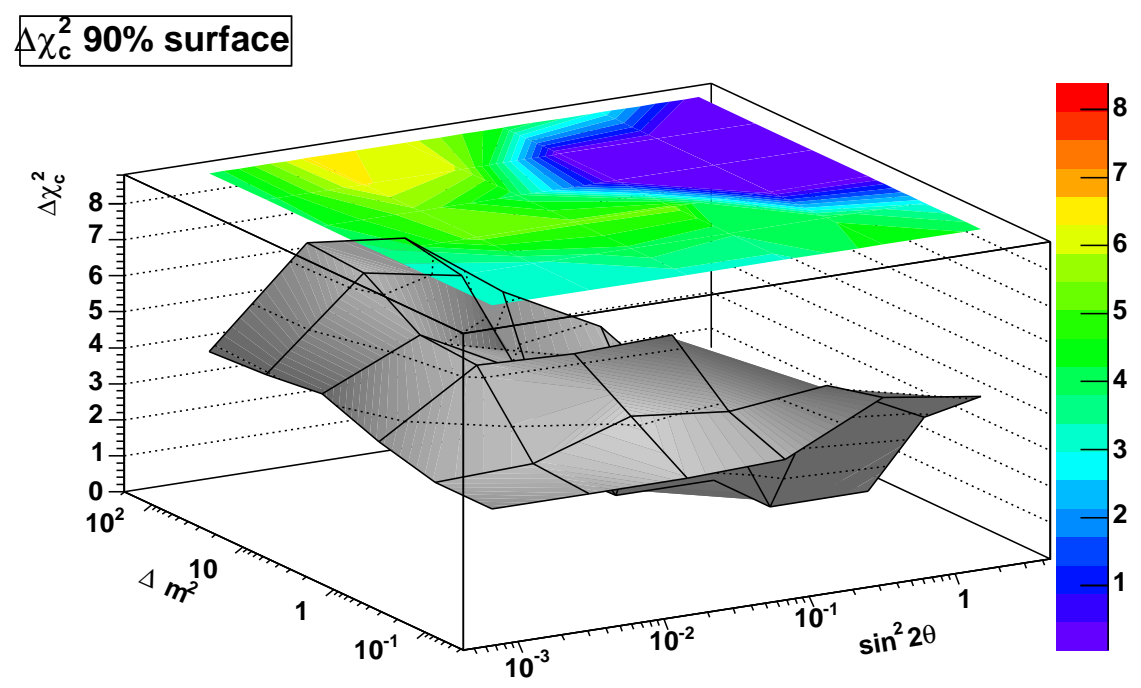

\section{$\chi^{\wedge} 2$ Distribution (grid)}

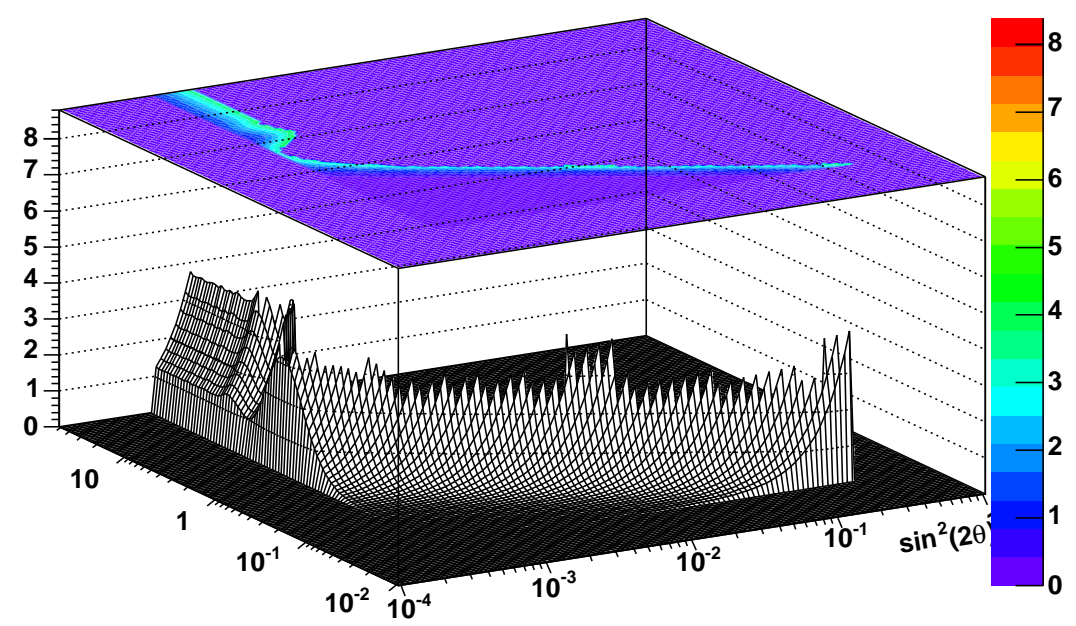

Figure C.4: Statistical errors only: Surface of the $\Delta \chi_{c}^{2}(90 \%)$ cut values that define the $90 \%$ C.L at each point in the parameter space. At the bottom, the intersection of the $\Delta \chi^{2}$ surface defined in Eq.(C.6) with a data set with no signal is shown yielding the $90 \%$ contour limit. 

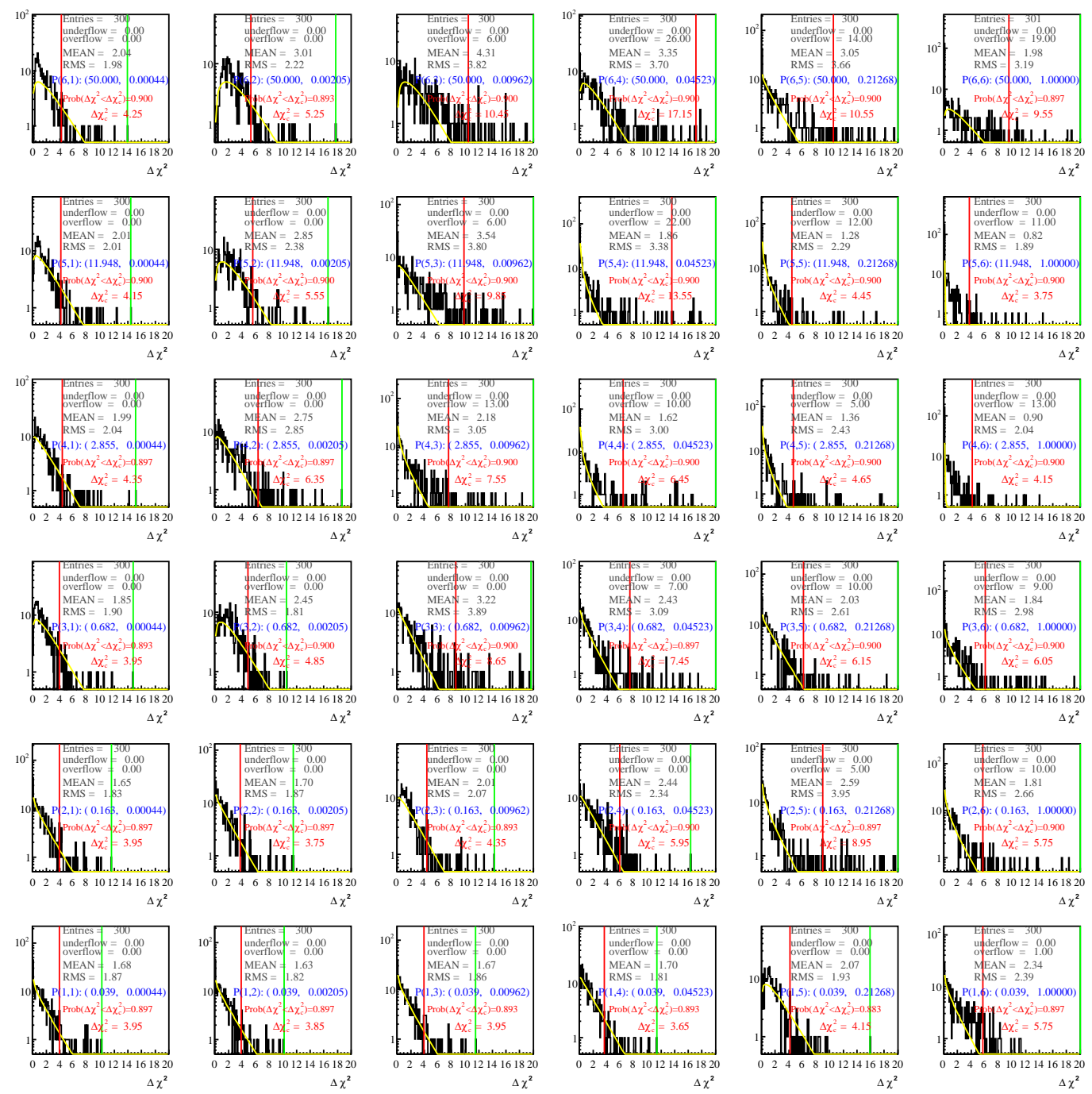

Figure C.5: Stat+Systematic errors: $\Delta \chi^{2}$ distributions for a grid of $6 \times 6$ points in the oscillations parameter space. 

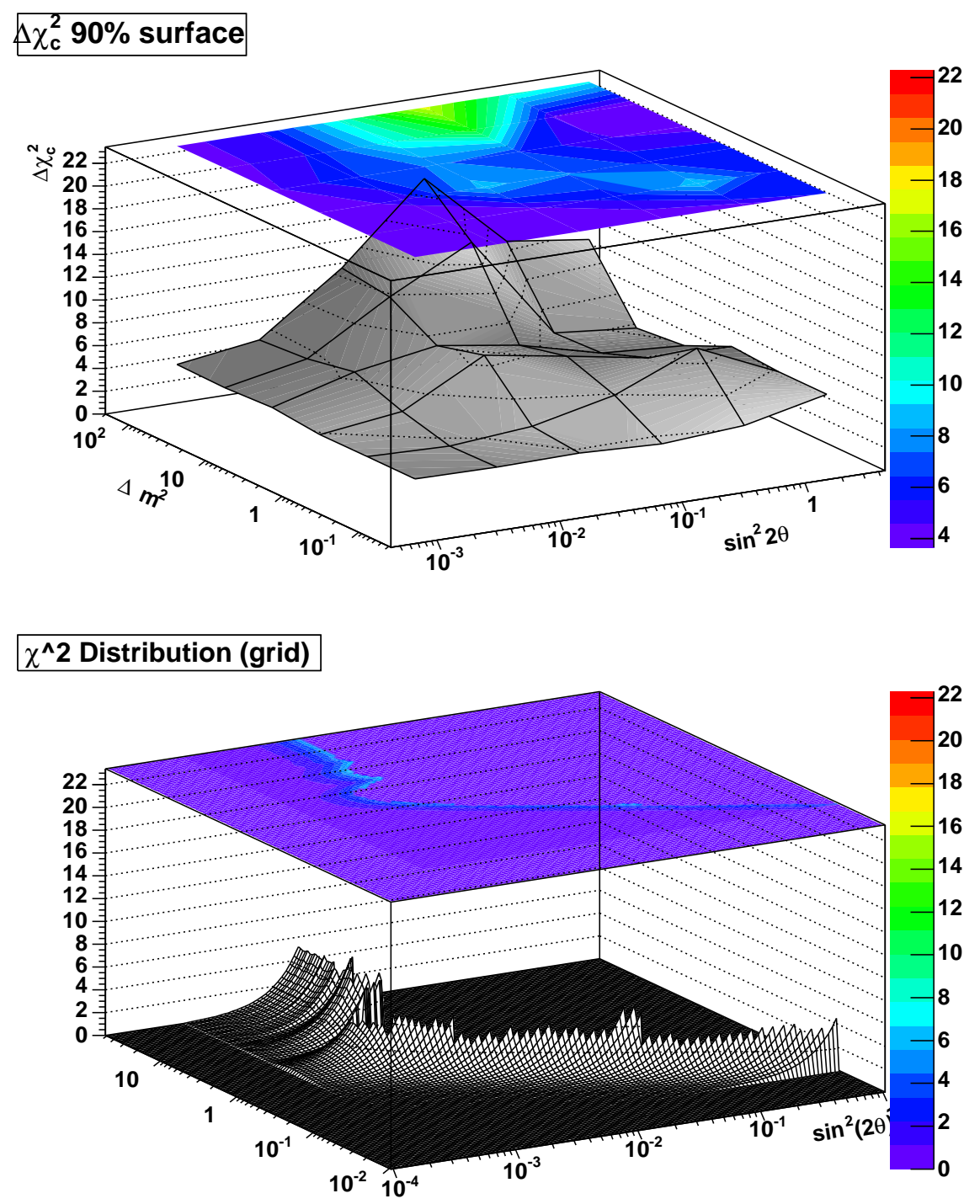

Figure C.6: Stat+Systematic errors: Surface of the $\Delta \chi_{c}^{2}(90 \%)$ cut values that define the $90 \%$ C.L at each point in the parameter space. At the bottom, the intersection of the $\Delta \chi^{2}$ surface defined in Eq.(C.6) with a data set with no signal is shown yielding the $90 \%$ contour limit. 


\section{C.6 Generalized Frequentist Sensitivity}

Schwetz [170] proposes that to obtain the sensitivity of an experiment such as MiniBooNE following a frequentist approach one could pose the following question:

Given a true point $\left(\sin ^{2} 2 \theta, \Delta m^{2}\right)$ in the parameter space, what is the probability that the null oscillations hypothesis results excluded at a certain confidence level?

Following [170] we will define the generalized sensitivity of the experiment as the set of all points $\left(\sin ^{2} 2 \theta, \Delta m^{2}\right)^{\text {true }}$ in the parameter space for which there is a $50 \%$ probability to exclude the null hypothesis $\left(\sin ^{2} 2 \theta=0\right)$ at a given C.L.

To answer this question we first find the value $\Delta \chi_{c}^{2}(n u l l ; \alpha)$ at which we keep $(1-\alpha) \times 100 \%(\alpha$ determines the C.L. we are interested on $)$ of the fake experiments whose true parameters are those of the null hypothesis. This value is given by the position of the lines in the lower left plot in Figure C.3 (Figure C.5) for some typical C.L.' ${ }^{4}$.

For each of the points in the grid and for each fake experiment generated at that point, we form the statistic:

$$
\left.D^{\prime}\right) \Delta \chi_{\text {null } i}^{2}=\chi^{2}\left(M_{B F_{i}}, P_{\text {null }}\right)-\chi^{2}\left(M_{B F_{i}}, P_{B F_{i}}\right), \quad i=1, \ldots, 1000
$$

this is, the difference in $\chi^{2}$ between the null hypothesis and the best fit point, using the matrix at the best fit point in both. Notice we have labeled this scheme $D^{\prime}$ ), for it is a variation of method the $D$ ) stated earlier.

\footnotetext{
4 To a good approximation. Note that the chosen grid does not include the null hypothesis points at $\sin ^{2} 2 \theta=0$, however, the smallest signal point in it is the one in the lower left corner.
} 
By counting the fraction of experiments in each grid point $P_{T}$ for which we obtain value of $\Delta \chi_{\text {null } i}^{2}$ larger than $\Delta \chi_{c}^{2}(n u l l ; \alpha)$, we calculate the probability

$$
\operatorname{Prob}\left[\Delta \chi_{\text {null } i}^{2}>\Delta \chi_{c}^{2}(\text { null } ; \alpha) \mid P_{T}\right]
$$

The 2D plot of these probabilities over all points $P_{T}$ on the oscillations parameter space contains all the information about how likely it is that the experiment will reject the null hypothesis at the $(1-\alpha) \times 100 \%$ C.L.

The generalized frequentist sensitivity curve is then obtained by taking the slice of this distribution at the value

$$
\operatorname{Prob}\left[\Delta \chi_{\text {null } i}^{2}>\Delta \chi_{c}^{2}(\text { null } ; \alpha) \mid P_{T}\right]=50 \%
$$

Using a $6 \times 6$ grid on the parameter space we formed the distributions of $\Delta \chi_{n u l l}^{2} i$ values for $i=1, \ldots, 300$ experiments at each point. These distributions are shown in Figure C.7 (Figure C.9) for the case of only statistical errors (statistical plus systematic errors). In each plot, the cut in $\Delta \chi_{\text {null c }}^{2}$ to keep $90 \%$ of the fake experiments when they are generated with the NULL signal is indicated with a red line, and is the same in all the plots in Figure C.7 (Figure C.9). It can be seen that when the fake data sets are generated with larger signals, it becomes more and more probable to exclude the NULL hypothesis at the given C.L. The probability to reject the NULL hypothesis at the $90 \%$ C.L. with only statistical errors (statistical plus systematic errors) is shown in Figure C.8(a) (C.10(a)) as a function of $\Delta m^{2}$ and $\sin ^{2} 2 \theta$.

The generalized definition of sensitivity advises us to take the slice at $50 \%$ probability and is shown in Figures C.8(b) and C.10(b).

It should be noted that the coarse grid used for this study is forcing us to make a very crude linear interpolation between the regions below and above $50 \%$ rejection probability. It is expected that the transition from low to high rejection probability 
will be very rapidly rising across this boundary, therefore the linear interpolation with so few grid points will give us the poor estimates shown here.

The comparison of our implementation of the generalized sensitivity method described in [170] with the result of the modified Feldman-Cousins technique is shown in Figure C.11 for both, statistical errors and statistical+systematic errors. The result of slicing the $\Delta \chi^{2}$ surface for the data with a constant plane at the level of 4.61 is shown for comparison. All three methods agree reasonably well, given the poor resolution of the grid that was used in the study. 


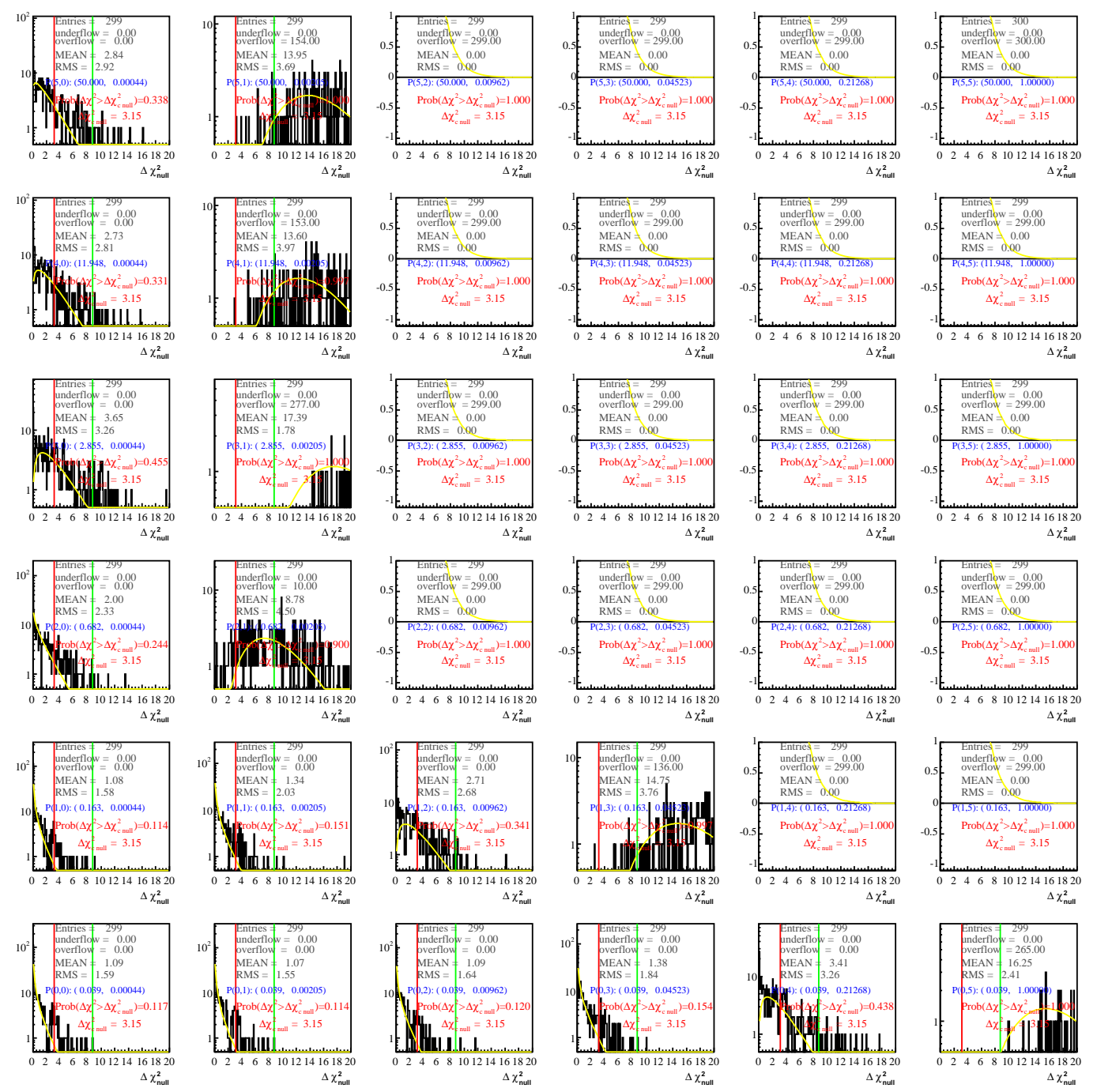

Figure C.7: Statistical errors: $\Delta \chi_{\text {null }}^{2}$ distributions for a grid of $6 \times 6$ points in the oscillations parameter space. The cut to keep $90 \%$ of the experiments when generated with the NULL signal point is indicated by a red line at $\Delta \chi_{\text {null }}^{2}=3.15$ in all the plots. As the signal becomes large, the probability to reject the NULL hypothesis grows. In the empty plots all the experiments have $\Delta \chi_{\text {null }}^{2}$ values larger than the displayed range. 


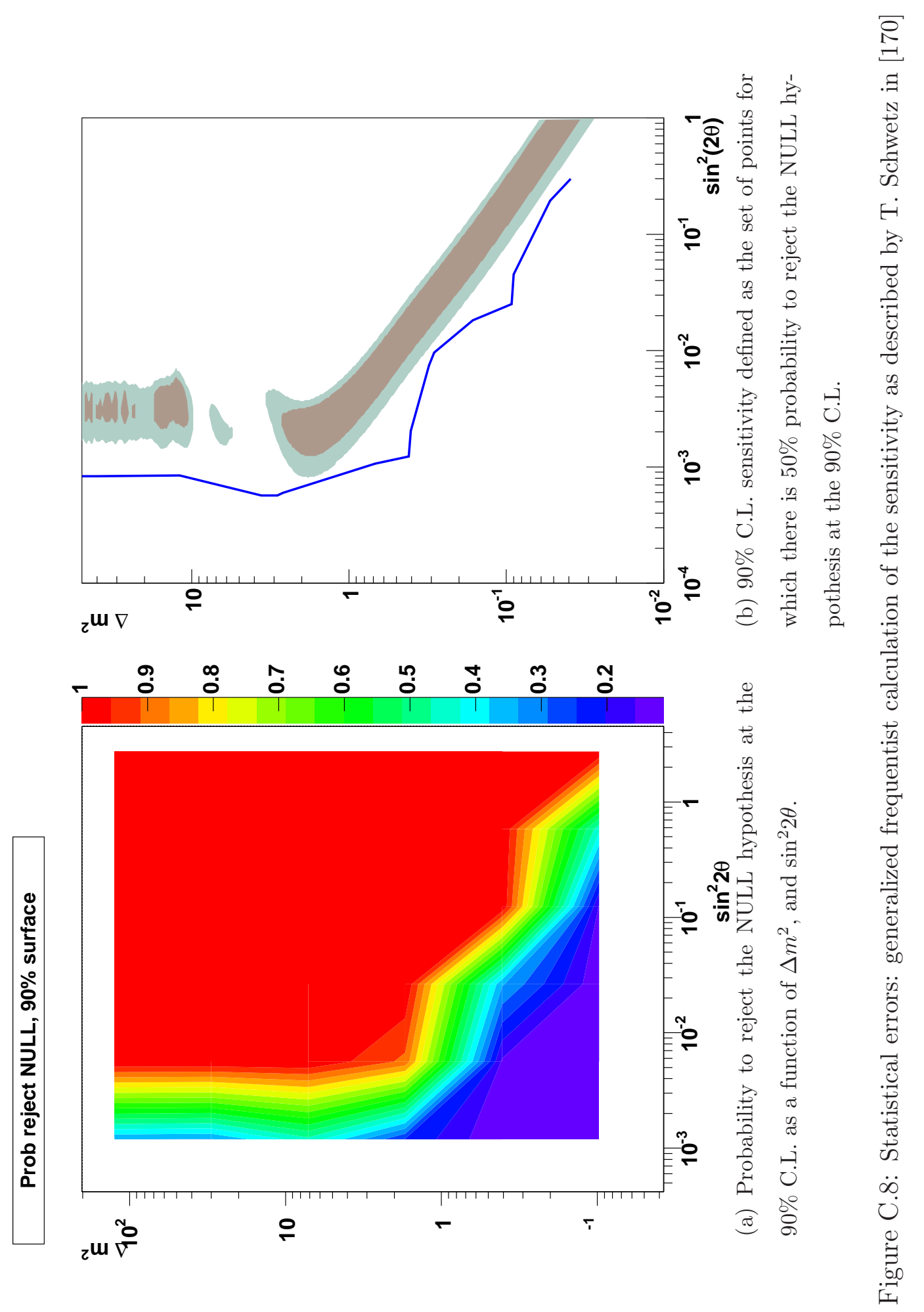



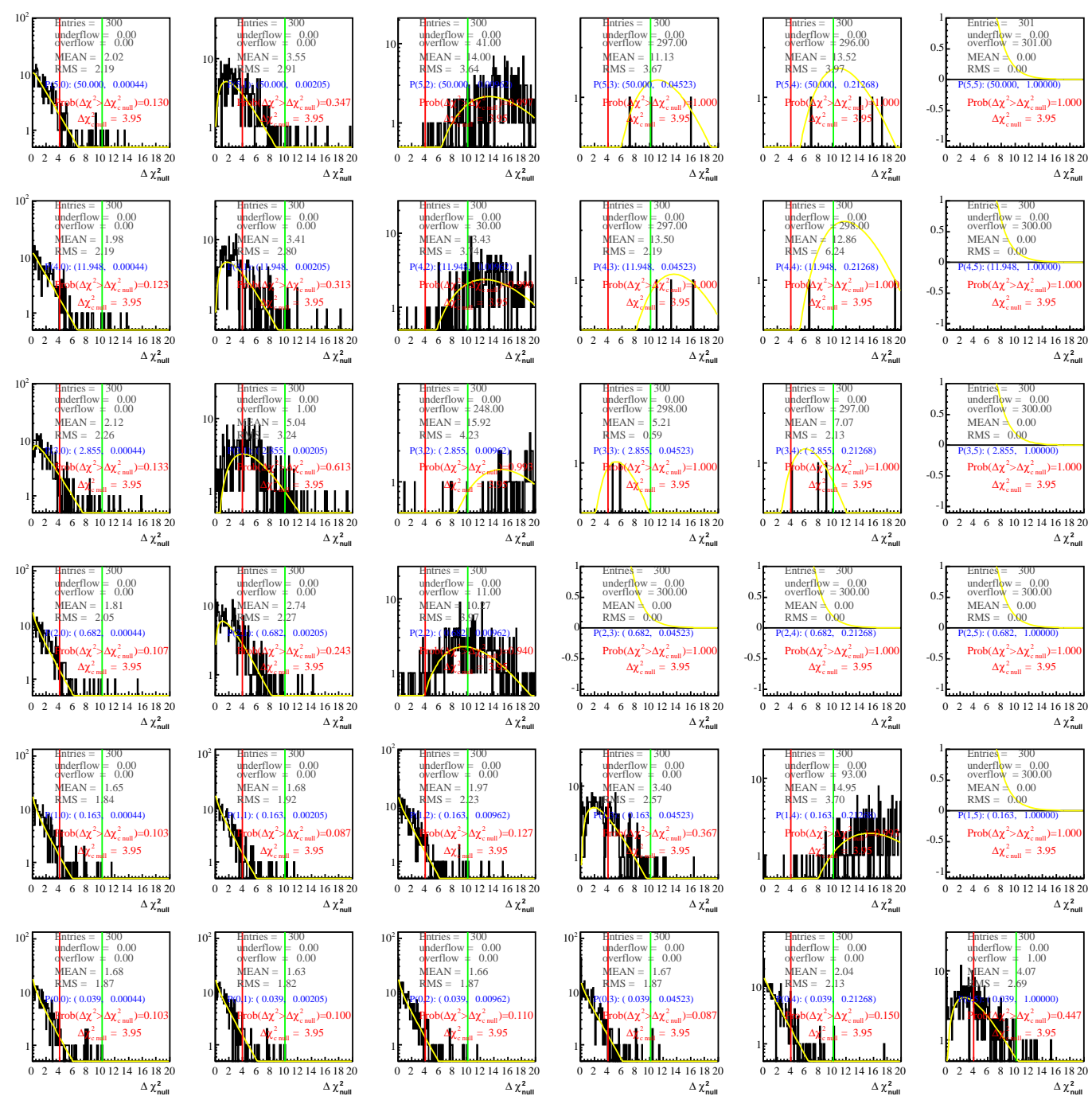

Figure C.9: Stat+Systematic errors: $\Delta \chi_{\text {null }}^{2}$ distributions for a grid of $6 \times 6$ points in the oscillations parameter space. The cut to keep $90 \%$ of the experiments when generated with the NULL signal point is indicated by a red line at $\Delta \chi_{\text {null }}^{2}=3.95$ in all the plots. As the signal becomes large, the probability to reject the NULL hypothesis grows. In the empty plots all the experiments have $\Delta \chi_{\text {null }}^{2}$ values larger than the displayed range. 


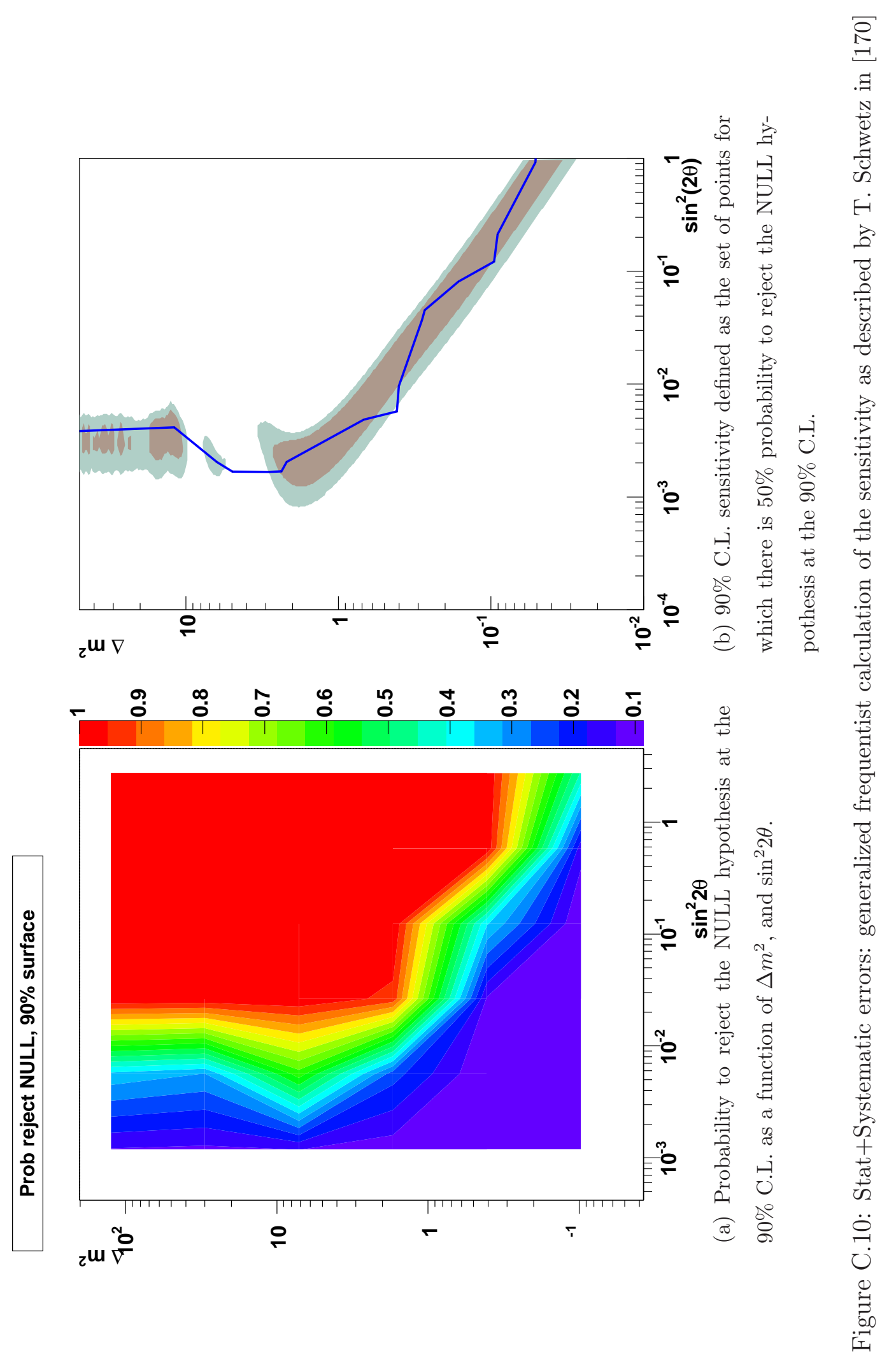




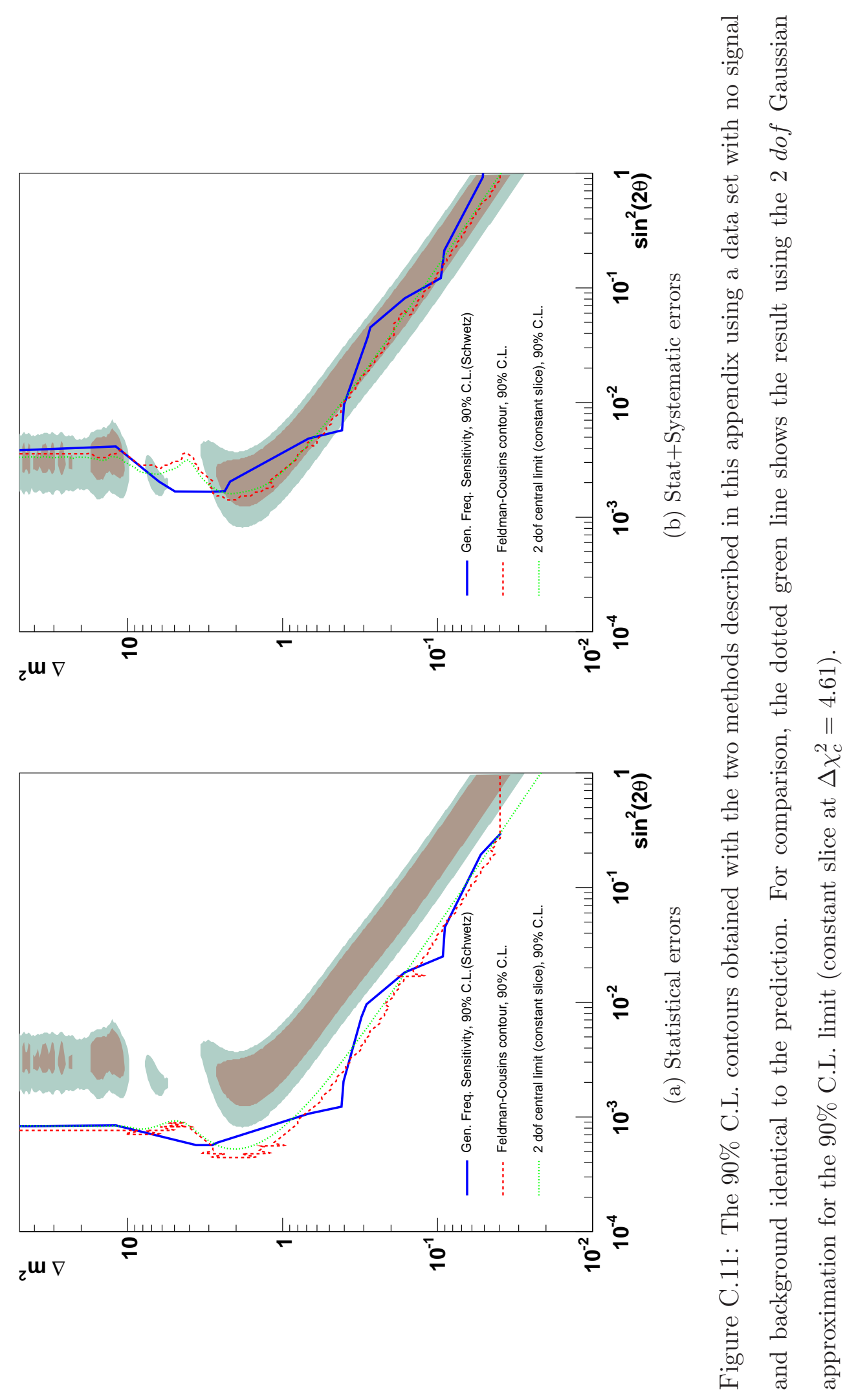




\section{Appendix D}

\section{Frequentist $3 \sigma$ and $5 \sigma$ C.L.}

\section{contours with limited numbers of fake experiments.}

\section{D.1 Introduction}

In Appendix $\mathrm{C}$ we used the CombinedFit package to produce multiple fake experiments with signals given by a set of selected points in the oscillation parameter space. The outcomes of such experiments as produced by the fitter code were used to construct the probability distribution of the $\Delta \chi^{2}$ statistic appropriate for each point, and then determine the cut in this statistic that would yield proper 90\% C.L. coverage. It is clear that in order to determine higher confidence intervals the number of fake experiments had to be much larger than those used in the study. The purpose of this appendix is to explore a method to estimate those higher confidence intervals based on the determination of the effective degrees of freedom for each true signal point, which can be extracted from the $90 \%$ C.L.'s, for which a smaller number of 
fake experiments is needed.

We will work under the assumption that the $\Delta \chi^{2}$ distributions obtained by running the CombinedFit package can be reasonably approximated by a Gamma Distribution, which can be looked at as a generalization of the familiar chisquare distribution allowing fractional degrees of freedom.

\section{D.2 The Gamma Distribution and the Incomplete Gamma Function}

We are interested in evaluating integrals of the Standard Gamma Distribution [172],

$$
f(x)=\frac{x^{\gamma-1} e^{-x}}{\Gamma(\gamma)} \quad x>0, \gamma>0
$$

where $\gamma$ is the real effective number of dof divided by 2 , and $\Gamma$ is the (complete) gamma function ${ }^{1}$. The cumulative distribution function (CDF) $F(x)$ of this $p d f$ is given in terms of the upper incomplete gamma function ${ }^{2}$ :

$$
F(x)=\Gamma_{x}(\gamma) / \Gamma(\gamma)
$$

ROOT v4.00/08 has a built in implementation of the incomplete gamma function [173] in the method TMath: : Gamma $(a, b)$ which, with the aid of a simple change of variables $(\gamma=2 a x=2 b)$ yields the cumulative gamma distribution in Eq. (D.2). This was implemented as shown in Figure D.1.

Shown in Table D.1 are the values obtained with this routine for the $\chi^{2}$ values for which the complementary cumulative gamma distribution $1-F\left(\chi^{2}\right)$ takes the value

\footnotetext{
${ }^{1} \Gamma(\gamma)=\int_{0}^{\infty} t^{\gamma-1} e^{-t} d t$

${ }^{2} \Gamma_{x}(\gamma)=\int_{0}^{x} t^{\gamma-1} e^{-t} d t$
} 


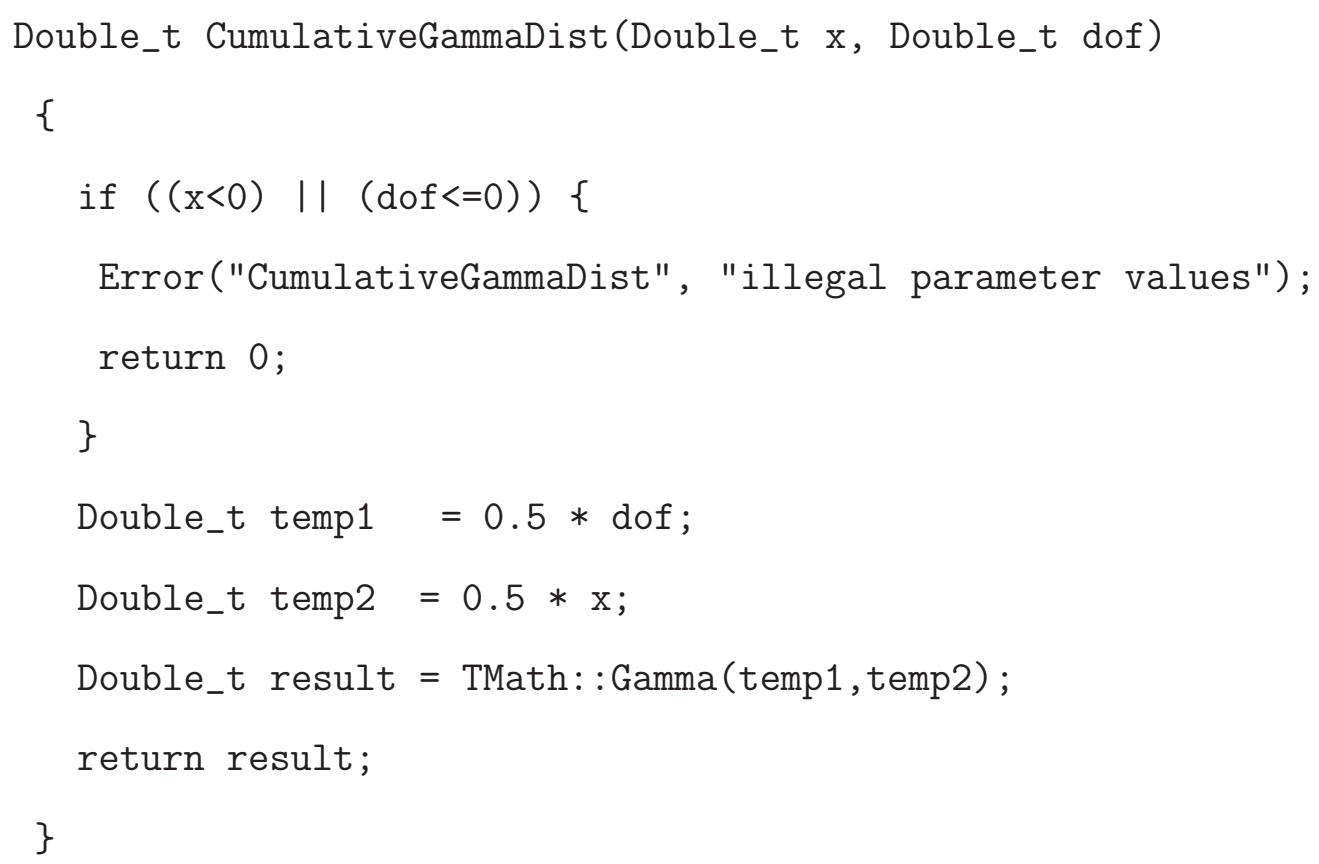

Figure D.1: Implementation of the change of variable to yield the CDF $F(x)$.

of some typically used probabilities. The table shows both, integer and fractional values for the parameter $\gamma$, which will be interpreted as the effective number of dof divided by 2 .

\section{D.3 Finding the effective number of $d o f$}

\section{D.3.1 Method I}

For each point in a grid of the oscillation parameter space and using the procedure outlined in Appendix $\mathrm{C}$, we form the distribution of $\Delta \chi^{2}$ values that the fitter returns. 
Table D.1: $\chi^{2}$ values defining the various central confidence intervals for different values of $\gamma=\operatorname{dof} / 2$ calculated with the above implementation of CumulativeGammaDist

\begin{tabular}{|c|c|c|c|c|c|c|c|c|c|}
\hline C.L. & $1-F\left(\chi^{2}\right)$ & 0.5 & 1 & 1.5 & 2 & 2.5 & 3 & 3.5 & $\leftarrow d o f$ \\
\hline $1 \sigma$ & 0.3173 & 0.334 & 1.00 & 1.658 & 2.296 & 2.917 & 3.527 & 4.127 & \\
\hline $2 \sigma$ & $4.5500 \mathrm{E}-02$ & 2.553 & 4.00 & 5.156 & 6.180 & 7.128 & 8.025 & 8.884 & \\
\hline $3 \sigma$ & $2.6998 \mathrm{E}-03$ & 7.041 & 9.00 & 10.51 & 11.83 & 13.03 & 14.16 & 15.23 & \\
\hline $4 \sigma$ & $6.3342 \mathrm{E}-05$ & 13.69 & 16.00 & 17.78 & 19.33 & 20.74 & 22.06 & 23.31 & \\
\hline $5 \sigma$ & 5.7330E-07 & 22.42 & 25.00 & 27.00 & 28.74 & 30.33 & 31.81 & 33.21 & \\
\hline $90 \%$ & 0.10000 & 1.500 & 2.706 & 3.704 & 4.605 & 5.448 & 6.251 & 7.026 & \\
\hline
\end{tabular}

We now fit to each of the distributions a function of the form ${ }^{3}$

$$
g(x)=p_{1} \times \frac{1}{2} x^{\left(p_{2} / 2-1\right)} e^{-x / 2}
$$

where the fit parameter $p_{1}$ takes care of the normalization and $p_{2} / 2$ corresponds to $\gamma$ in Eq.(D.1). Naively one would integrate the fitted function from 0 to $\Delta \chi_{c}^{2}$ such that the integral yields $0.9973(3 \sigma)$, or $0.99999943(5 \sigma)$. To avoid numerical integration problems one instead resorts to the equivalent method of using the cumulative gamma distribution as described in Section D.2, with dof $=p_{2}$.

The $3 \sigma$ and $5 \sigma$ contours will be determined by finding the appropriate cut values $\Delta \chi_{c}^{2}(\alpha)$, for $(1-\alpha) \times 100 \%$ C.L., such that the cumulative gamma distribution satisfies:

$$
\begin{array}{rc}
F\left(\Delta \chi_{c}^{2}(1.0000 E-01)\right)=0.9 & 90 \% \text { C.L. } \\
F\left(\Delta \chi_{c}^{2}(2.6998 E-03)\right)=0.9973 & 3 \sigma \text { C.L. } \\
F\left(\Delta \chi_{c}^{2}(5.7330 E-07)\right)=0.99999943 \quad 5 \sigma \text { C.L. }
\end{array}
$$

\footnotetext{
${ }^{3}$ Note that the factor of $1 / 2$ in the exponential is what introduced the need for the change of variable $x \longrightarrow 0.5 x$ in the implementation of CumulativeGammaDist of Figure D.1
} 
at each point in the grid.

\section{D.3.2 Method II}

One can also obtain the effective number of dof without performing a fit to a gamma distribution pdf at each grid point. Instead, we just find the cut for the 90\% C.L. directly from the $\Delta \chi^{2}$ distributions and ask the question

Given $\Delta \chi_{c}^{2}$ such that $90 \%$ of the experiments in a particular grid point have $\Delta \chi^{2}<\Delta \chi_{c}^{2}$, what is the value of $\gamma=$ dof $/ 2$ for which $F\left(\Delta \chi_{c}^{2}\right)=0.9$ ?

This will also make the assumption that the distributions of $\Delta \chi^{2}$ values are gamma distributions, but without the need of performing a fit.

Suppose that we only had of order $\sim 10^{3}$ experiments for each point at our disposal over a $20 \times 20 \operatorname{grid}^{4}$. Then one can obtain the $90 \%$ C.L. cut in the $\Delta \chi^{2}$ statistic with an accuracy of $\sim 10 \%$. This would be the level of precission with which our effective number of dof would be determined.

Once the effective number of $d o f$ is determined we proceed to find the $\Delta \chi_{c}^{2}$ values for the $3 \sigma$ and $5 \sigma$ as done in Method I.

\section{D.3.3 Comparison of Methods I and II}

Table D.2 shows the comparison of the effective number of dof found using methods I and II for the set of points defined by the $6 \times 6$ grid used in Appendix C. There were 400 experiments at each point, therefore the numbers for Method II are estimated to have an uncertainty in the number of $d o f$ of $\sim 15 \%$.

\footnotetext{
4 This corresponds to a total of $500 \times 20 \times 20=200$, 000 fits.
} 
Table D.2: Comparison of $d o f_{\text {eff }}$ obtained with Methods I and II. See Appendix C for the location of the points. Numbers from Method II have an uncertainty of $\sim 15 \%$

\begin{tabular}{|c|c|c|c|c|c|c|c|c|}
\hline Point & I & II & Point & I & II & Point & I & II \\
\hline$\left(\begin{array}{ll}1 & 1\end{array}\right)$ & 1.81 & 1.85 & $\left(\begin{array}{ll}3 & 1\end{array}\right)$ & 2.51 & 1.85 & $\left(\begin{array}{ll}5 & 1\end{array}\right)$ & 2.59 & 2.00 \\
\hline$\left(\begin{array}{ll}1 & 2\end{array}\right)$ & 1.78 & 1.85 & $\left(\begin{array}{ll}3 & 2\end{array}\right)$ & 2.85 & 2.45 & $\left(\begin{array}{l}5 \\
2\end{array}\right)$ & 2.99 & 2.60 \\
\hline$\left(\begin{array}{ll}1 & 3\end{array}\right)$ & 1.81 & 1.85 & $\left(\begin{array}{ll}3 & 3\end{array}\right)$ & 2.00 & 3.40 & $\left(\begin{array}{ll}5 & 3\end{array}\right)$ & 2.39 & 6.30 \\
\hline$\left(\begin{array}{ll}1 & 4\end{array}\right)$ & 2.07 & 1.85 & $\left(\begin{array}{ll}3 & 4\end{array}\right)$ & 1.78 & 3.40 & $(54)$ & 0.59 & 13.60 \\
\hline$(15)$ & 2.56 & 2.15 & (3 5) & 1.46 & 3.55 & $(55)$ & 0.36 & 2.60 \\
\hline$\left(\begin{array}{ll}1 & 6\end{array}\right)$ & 1.56 & 2.90 & $(36)$ & 0.86 & 2.30 & $(56)$ & 1.00 & 0.95 \\
\hline$\left(\begin{array}{ll}2 & 1\end{array}\right)$ & 1.78 & 1.70 & $\left(\begin{array}{ll}4 & 1\end{array}\right)$ & 2.20 & 2.00 & $\left(\begin{array}{ll}6 & 1\end{array}\right)$ & 2.88 & 2.00 \\
\hline$\left(\begin{array}{l}2 \\
2\end{array}\right)$ & 2.00 & 1.85 & $\left(\begin{array}{ll}4 & 2\end{array}\right)$ & 2.44 & 2.60 & $\left(\begin{array}{ll}6 & 2\end{array}\right)$ & 3.45 & 2.60 \\
\hline$\left(\begin{array}{ll}2 & 3\end{array}\right)$ & 2.42 & 2.15 & (4 3) & 1.27 & 5.40 & $\left(\begin{array}{lll}6 & 3\end{array}\right)$ & 3.43 & 6.85 \\
\hline$(24)$ & 2.02 & 2.75 & $\left(\begin{array}{ll}4 & 4\end{array}\right)$ & 0.56 & 4.40 & $\left(\begin{array}{ll}6 & 4\end{array}\right)$ & 2.41 & 15.45 \\
\hline$(25)$ & 1.38 & 2.30 & $(45)$ & 0.58 & 1.55 & $(65)$ & 1.87 & 8.00 \\
\hline$(26)$ & 1.23 & 2.90 & $(46)$ & 1.00 & 1.05 & $\left(\begin{array}{l}6 \\
6\end{array}\right)$ & 1.00 & 6.15 \\
\hline
\end{tabular}


It should be noted that points $(4,6)$ and $(6,6)$ in the table (at $\sin ^{2} 2 \theta=1.0$ ) were assigned $d o f=1.00$ by Method I solely because the fit to a gamma distribution failed. In most of the cases the effective number of dof is reasonably close. Examples of some fits are shown in Figure D.2.

The comparison of the numbers in Table D.2 is better appreciated in Figure D.3. Notice that in the regions where the sensitivity of the experiment is expected the two methods have very similar values of the effective number of dof. Only in the case of a strong signal with $\Delta m^{2} \sim 1 \mathrm{eV}^{2}$ or larger the contours obtained with the two methods are expected to disagree.

It is interesting to note that the region of large disagreement between the two methods is where strong non-linearities are expected in the oscillation parameter space due to the periodic nature of the oscillation probability that is more apparent at high values of $\Delta m^{2}$. These are regions where the $\Delta \chi^{2}$ distributions are not well described by the gamma distribution and have long tails, giving small dof values from the fits in Method I and large values from the counting of experiments in the tails of the distributions in Method II.

The Feldman-Cousins contours obtained with the two methods when applied to the final MiniBooNE data sample ${ }^{5}$ are shown in Figure D.4. Also shown are the contours obtained using the global scan (constant slice) method. A it is here, it is usually the case that higher confidence intervals are in better agreement between the global scan and Feldman-Cousins methods.

${ }^{5}$ In this appendix we used the BDT algorithm of Section 3.5.2 that was used for the first MiniBooNE result in Ref.[190], and all the corresponding systematic uncertainties. 


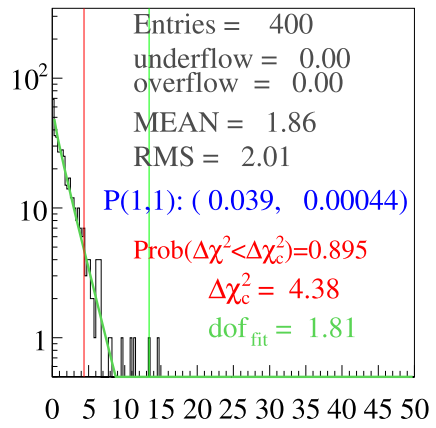

$\Delta \chi^{2}$

(a) Stat+Systematic,point $\left(\Delta m^{2}, \sin ^{2} 2 \theta\right)=(0.039,0.00044)$

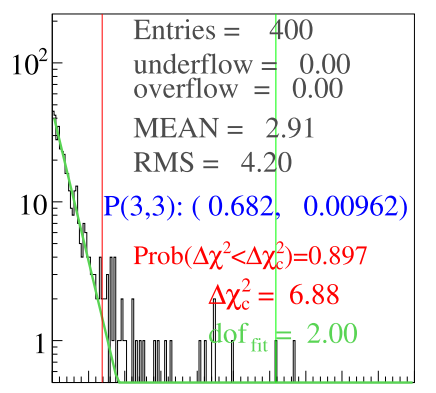

$0 \quad 5101520253035404550$

$\Delta \chi^{2}$

(d) Stat+Systematic,point

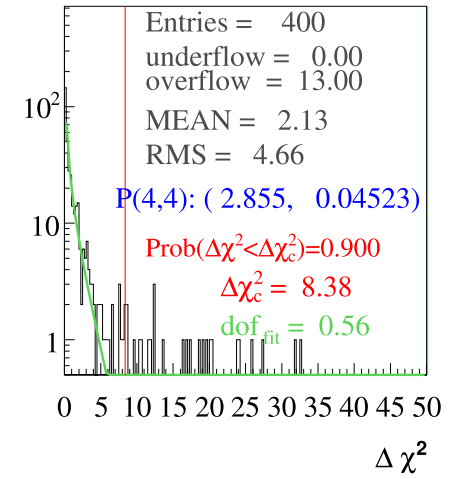

(b) Stat+Systematic,point

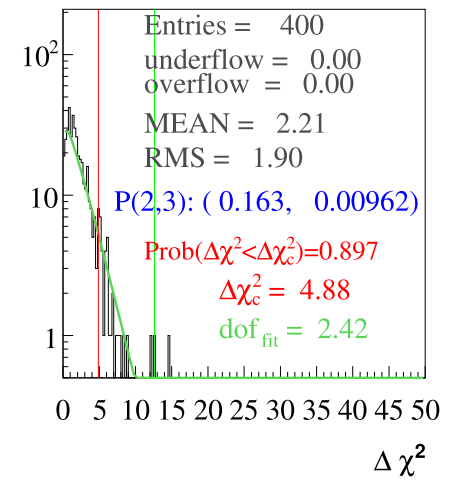

(c) Stat+Systematic,point

$\left(\Delta m^{2}, \sin ^{2} 2 \theta\right)=(2.855,0.04523)$

$\left(\Delta m^{2}, \sin ^{2} 2 \theta\right)=(0.163,0.00962)$
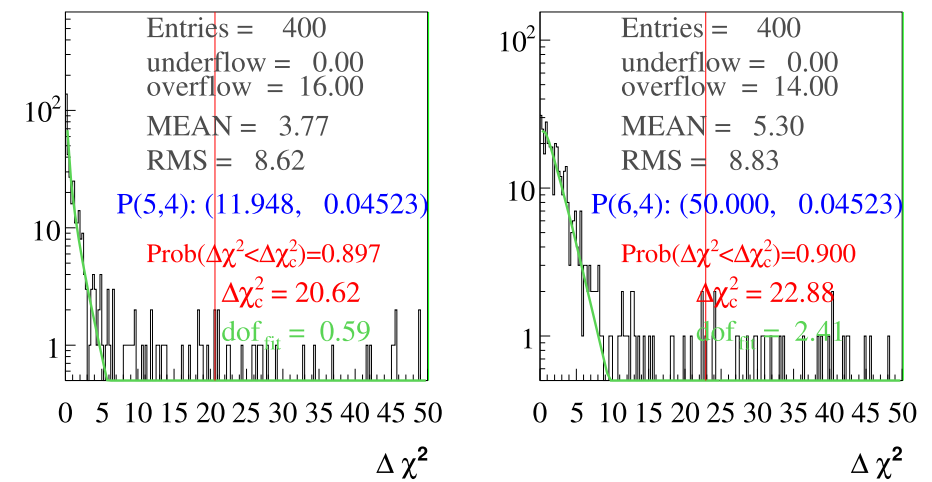

(e) Stat+Systematic,point

(f) Stat+Systematic,point

$\left(\Delta m^{2}, \sin ^{2} 2 \theta\right)=(0.682,0.00962)\left(\Delta m^{2}, \sin ^{2} 2 \theta\right)=(11.948,0.04523)\left(\Delta m^{2}, \sin ^{2} 2 \theta\right)=(50.0,0.04523)$

Figure D.2: $\Delta \chi^{2}$ distributions for six points in the grid used in Appendix C. The solid green line is the fit used in Method I. The quoted values of $\Delta \chi_{c}^{2}$ are for the $90 \%$ C.L. that is used in Method II. 


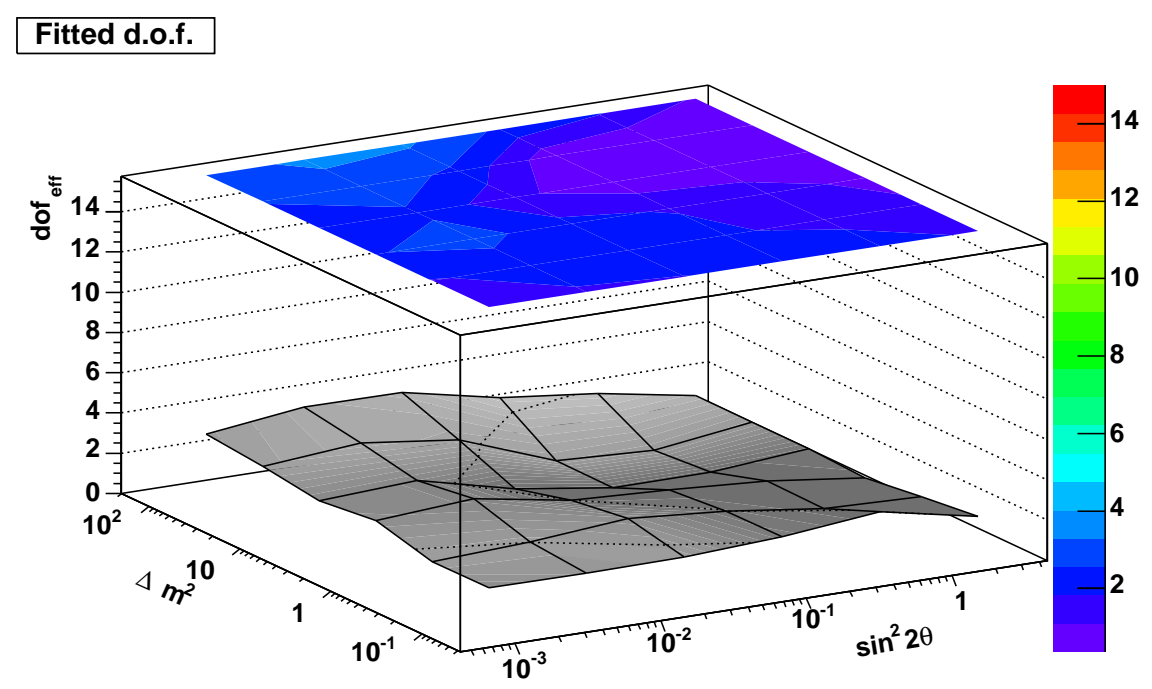

\section{Estimated d.o.f.}

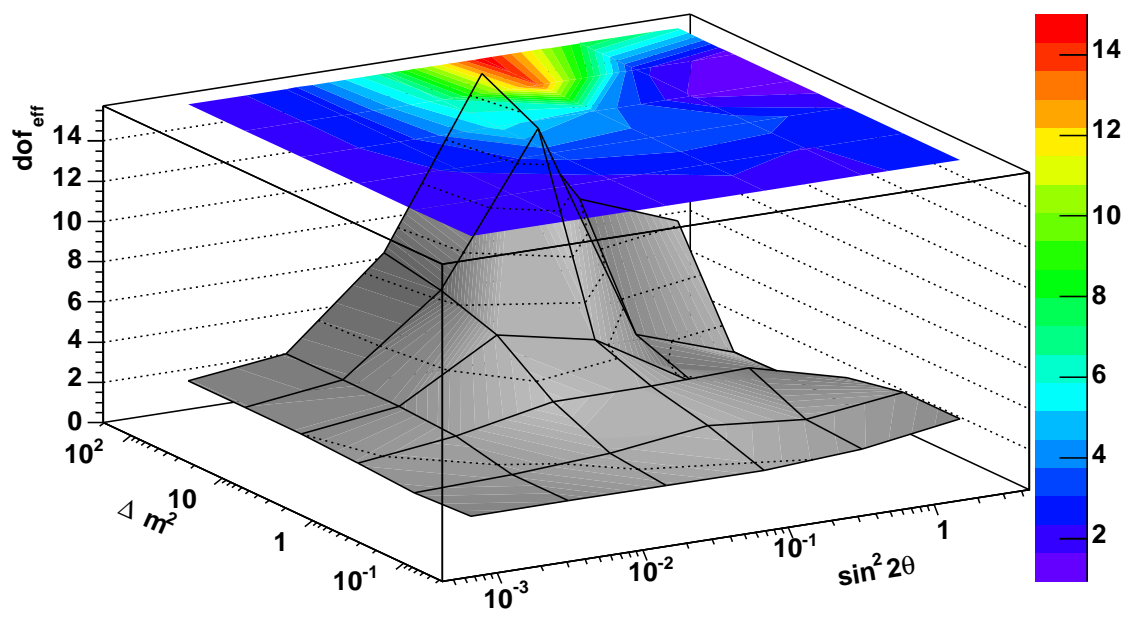

Figure D.3: Effective number of dof from Method I (top), and from method II (bottom) 


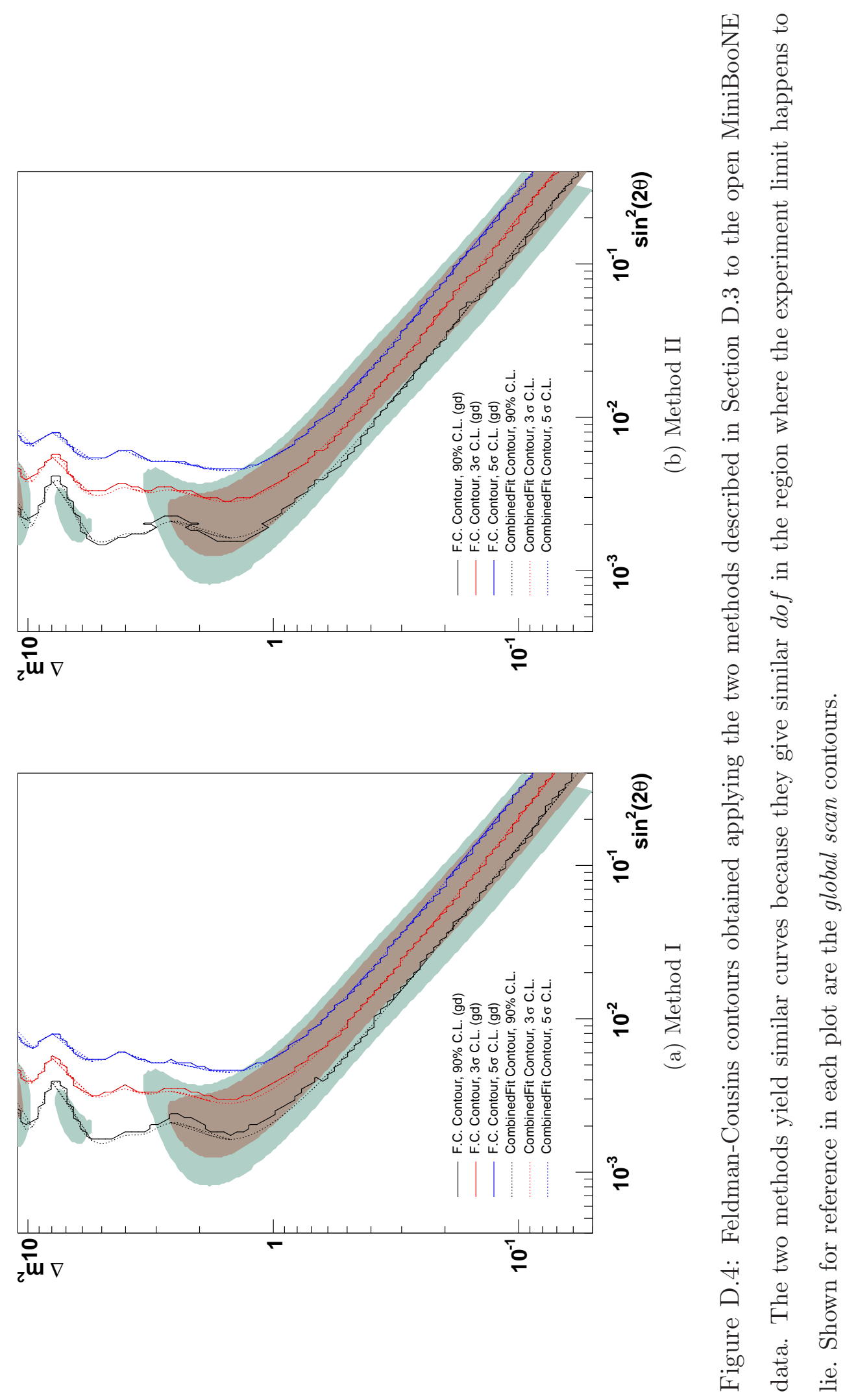




\section{D.4 Conclusions}

An approximate method to calculate $3 \sigma$ and $5 \sigma$ contours with low numbers of fake experiments using the unified approach of Feldman and Cousins based on a calculation of the effective number of dof has been implemented. Two alternative methods to calculate the effective number of dof have been used and shown to yield similar results in the region where the sensitivity of the experiment lies. 


\section{Appendix E}

\section{Frequentist contours for the MiniBooNE BDT analysis.}

\section{E.1 Introduction}

In this Appendix we show the results of the methods described in Appendix D to the BDT oscillation analysis.

A total of 1011 fake experiments were run for each of 400 points in a $20 \times 20$ grid in the oscillation parameter space ${ }^{1}$. The grid points are chosen to be equally spaced in logarithmic scale, and correspond to a subset of the $120 \times 120$ grid that was used to perform each fit using the CombinedFit package.

The curves for the Feldman-Cousins method shown here correspond to those obtained with Method I in Appendix D. The small differences with the result obtained with Method II in the region of interest are considered not to be a concern.

\footnotetext{
1 The 404,400 fits were run over a period of 9 days in the MiniBooNE Condor cluster, submitting groups of 400 fits in individual jobs (1011 Condor jobs). Each job took an average time of $\sim 4.5$ hrs. to complete and ran typically $\sim 20$ simultaneous jobs.
} 
The results presented here can be directly compared to those presented in Section 4.3 for the BDT analysis.

\section{E.2 Results}

Figure E.1 Shows the comparison of the contours obtained with the global scan method (constant slice), the unified approach of Feldman-Cousins, and the generalized frequentist sensitivity of T. Schwetz described in Appendices C and D. The Schwetz method gives a significantly different result for the $3 \sigma$ and $5 \sigma$ contours when compared to the other two. At this point this difference is not understood, but it is likely to improve with a more refined grid.

The effect of the size of the grid that is used to determine the surface of $\Delta \chi_{c}^{2}$ cut values in the Feldman-Cousins method is small because the gradients over this surface are not large in the regions of interest. It should be noted that it also uses the more refined information in the $\chi^{2}$ surface from the fit to the data (a $120 \times 120$ grid). Even with a much smaller grid as the $6 \times 6$ used in Appendix D reasonable results are obtained.

Figure E.2 shows the comparison of the Feldman-Cousins and global scan methods with the raster scan technique implemented in CombinedFit to make a 1-dimensional fit of $\sin ^{2} 2 \theta$ for each $\Delta m^{2}$ value. The $90 \%$ C.L. curve for this technique used a $\Delta \chi^{2}=1.64$ that corresponds to a 1 -sided Gaussian distribution $\left(\sin ^{2} 2 \theta\right.$ cannot be negative). The $3 \sigma$ and $5 \sigma$ contours used the corresponding values for a 2-sided Gaussian distribution $\left(\Delta \chi^{2}=9\right.$, and $\Delta \chi^{2}=25$ respectively). We also chose to show the 90\% C.L. region from the joint KARMEN-LSND analysis [101] here.

The $3 \sigma$ and $5 \sigma$ contours from the global scan (inherently 2-dimensional because it used $\Delta \chi^{2}=11.83$, and 28.67 respectively) are consistent with those from the 
raster scan (inherently 1 -dimensional, $\Delta \chi^{2}=9,25$ respectively) because the right $\Delta \chi^{2}$ value is chosen in each case. It can be inferred from the $90 \%$ C.L. curves that a $\Delta \chi^{2}=2.71$ (1-sided, $1-d o f$ for $90 \%$ C.L.) will be close to the global scan result as well.

\section{E.3 Effective number of $d o f$}

Figure E.3 shows the effective number of dof calculated using the two methods described in Appendix D. These surfaces are relevant to the discussion of how to approximate the $3 \sigma$ and $5 \sigma$ contours with a limited number of fake experiments, since the $90 \%$ C.L. can be safely estimated with $\sim \mathcal{O}(1000)$ experiments using the distributions of $\Delta \chi^{2}$ themselves. It can be seen that in the region where the MiniBooNE limits lie the two methods give similar effective numbers of $\operatorname{dof}(\sim 2)$. This is supported by the fact that the extracted contours agree well with the results from the global scan. 


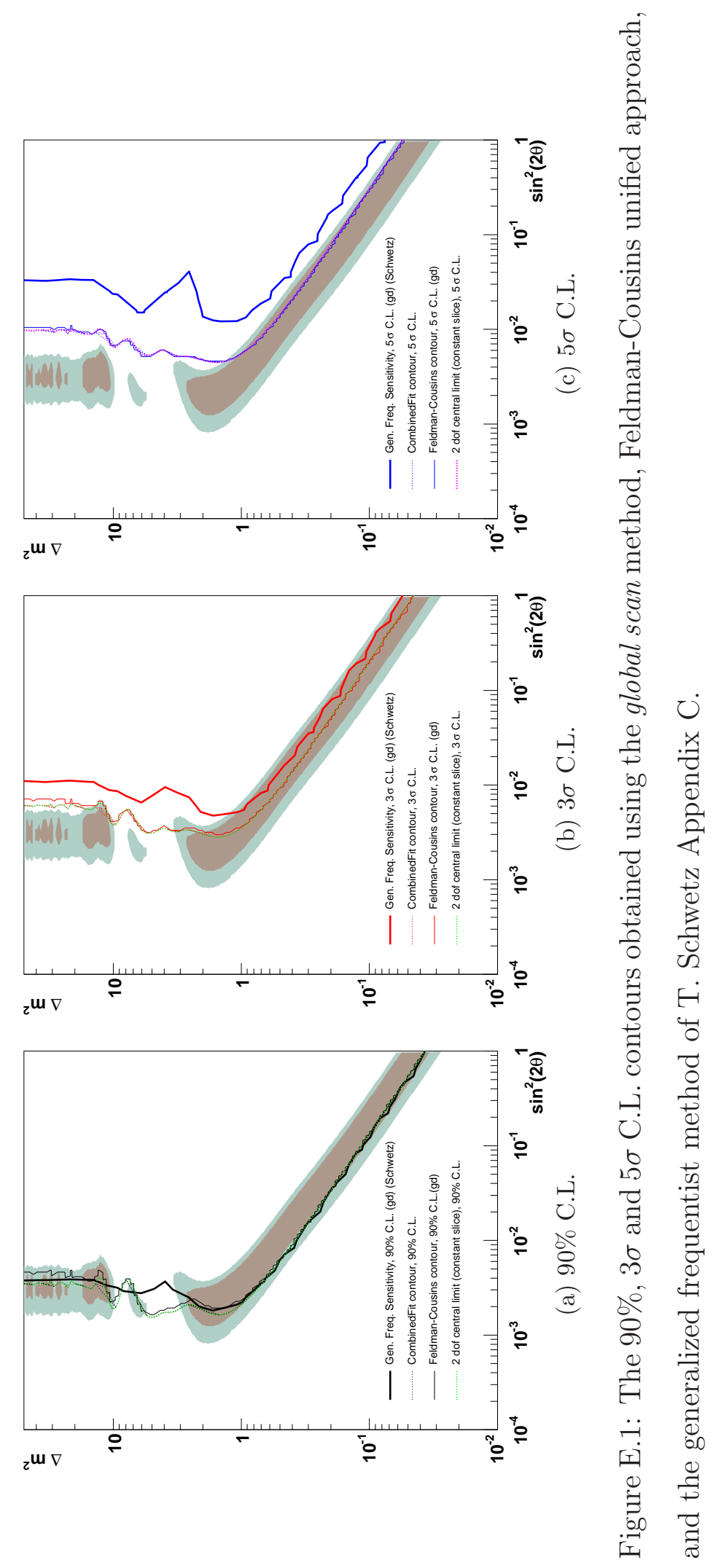




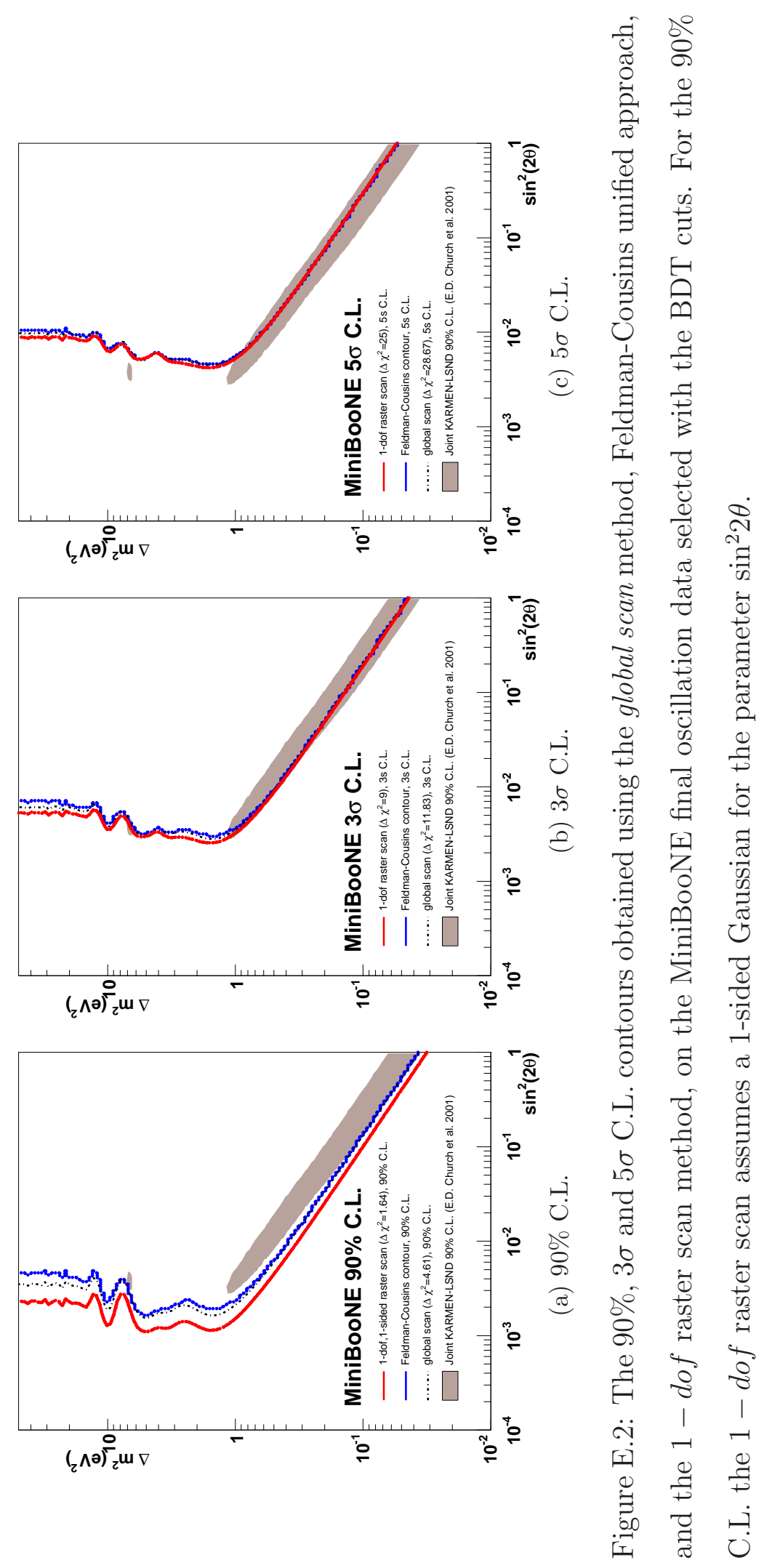




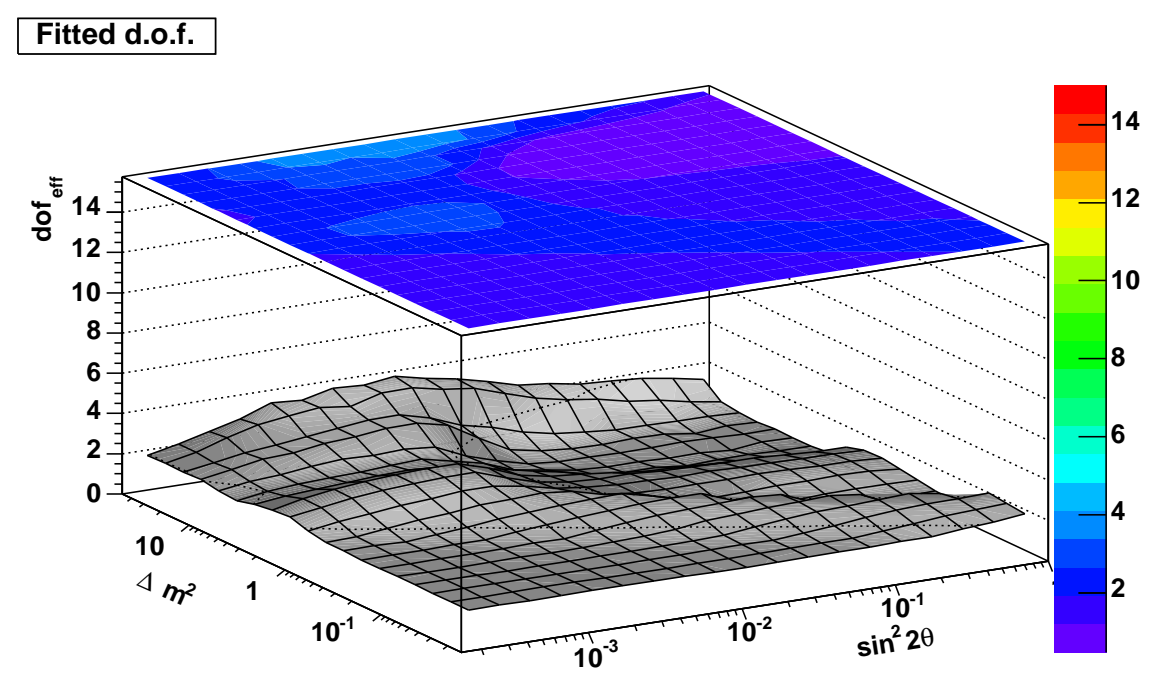

\section{\begin{tabular}{|l|}
\hline Estimated d.o.f. \\
\hline
\end{tabular}}

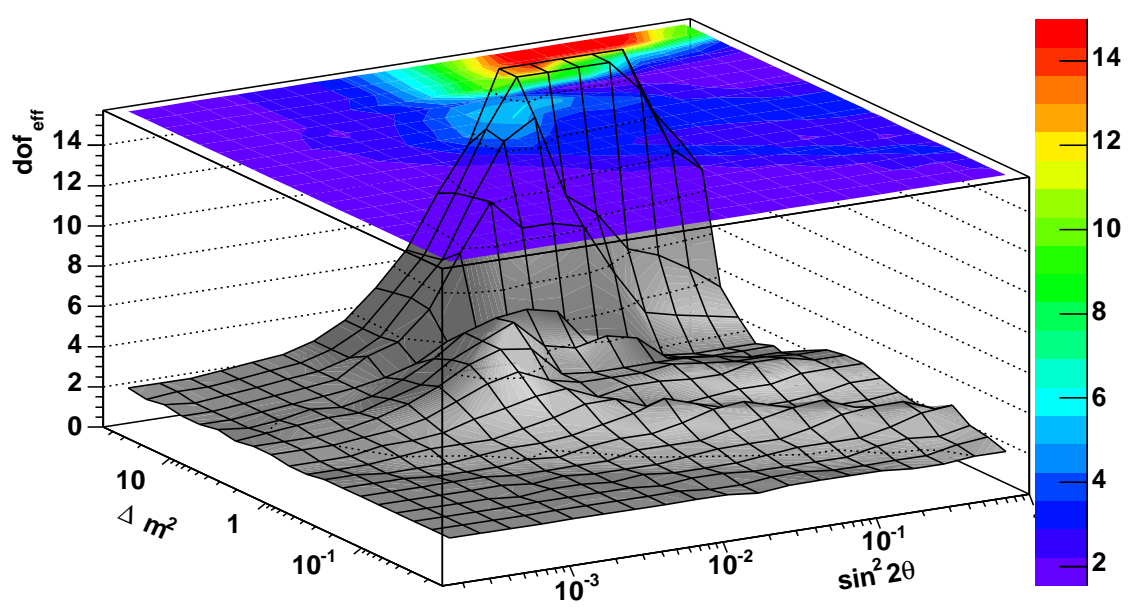

Figure E.3: Effective number of degrees of freedom calculated using Methods I (top) and II (bottom ) described in Appendix D with the $20 \times 20$ grid. The Feldman-Cousins contours in this note were calculated using Method I. Method II yields very similar results by virtue of the specific location of the MiniBooNE limits in this space. 


\section{Appendix F}

\section{Miscellaneous tests of the fits}

\section{F.1 Tests of statistical correlations with system- atic errors}

Here we show a collection of fits to a fake input signal used to test the handling of statistical correlations in the fitting code when all systematic uncertainties are included.

These tests consist of three steps:

I: Perform a $\nu_{e} / \nu_{\mu}$ combined fit.

II: Perform a $\nu_{e} / \nu_{e} / \nu_{\mu}$ combined fit in which the two electron samples are the same sample ignoring statistical correlations. II: Perform a $\nu_{e} / \nu_{e} / \nu_{\mu}$ combined fit in which the two electron samples are the same sample. Including the statistical correlations

The test is passed successfully if the results of I and III are the same.

We first consider the systematic uncertainties that include the smoothed O.M. 
errors and later the unsmoothed O.M. errors, both of which are described in described in 4.4.1.

The cautious reader will have realized that in these tests the total error matrix is a perfectly singular matrix, (the top half of the matrix is identical to its lower half). These tests, including the tests using only statistical errors in Section 4.5, were performed by forcing the total error matrices to be non-singular by adding a small ${ }^{1}$ number $\epsilon$ to the lower diagonal. It was observed that the agreement of steps I and III in the tests increased as $\epsilon$ became smaller.

The results are arranged as follows:

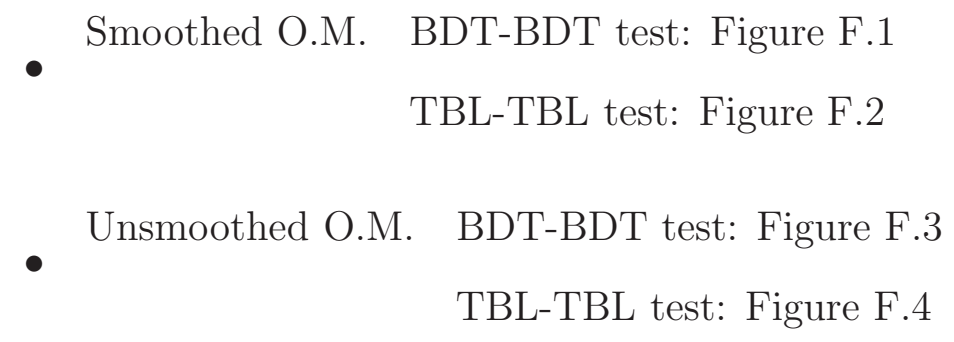

Note that in the case of unsmoothed O.M. errors it is not possible to ignore the statistical correlations, since they are expected to be contained in the O.M. error component. The tests in Figure F.3 and Figure F.4 indicate that the statistical correlations between the various bins in the unsmoothed O.M. matrix are not bad approximations for the TBL case, while they are not so good for the BDT case.

\footnotetext{
${ }^{1}$ Compared to the eigenvalues of the matrix.
} 

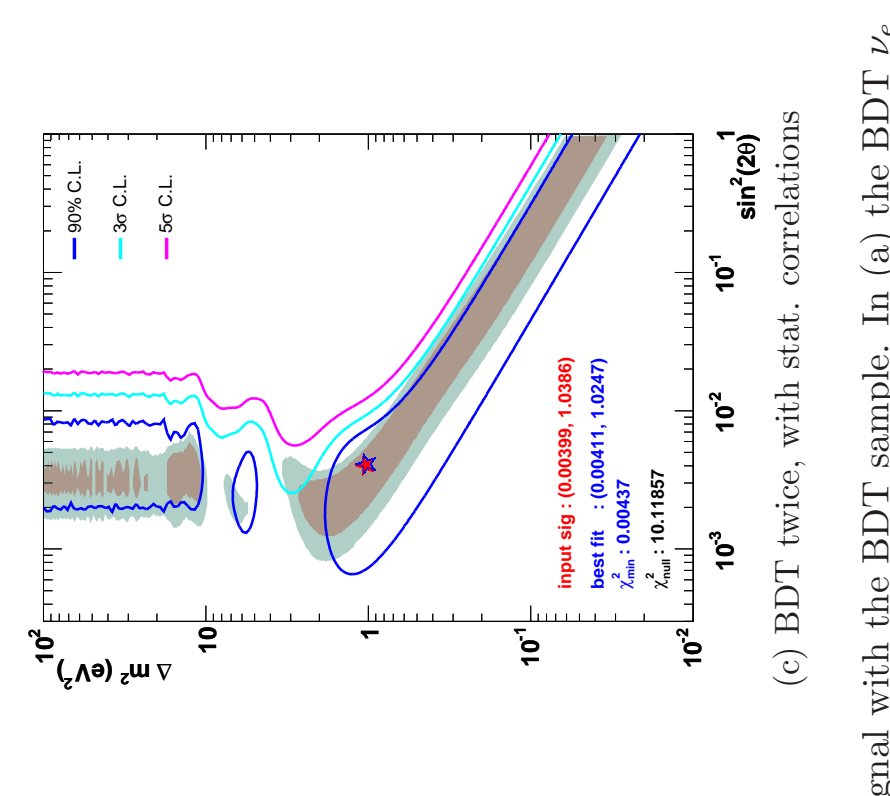

^

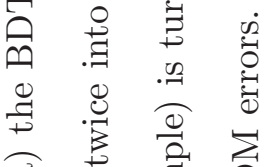

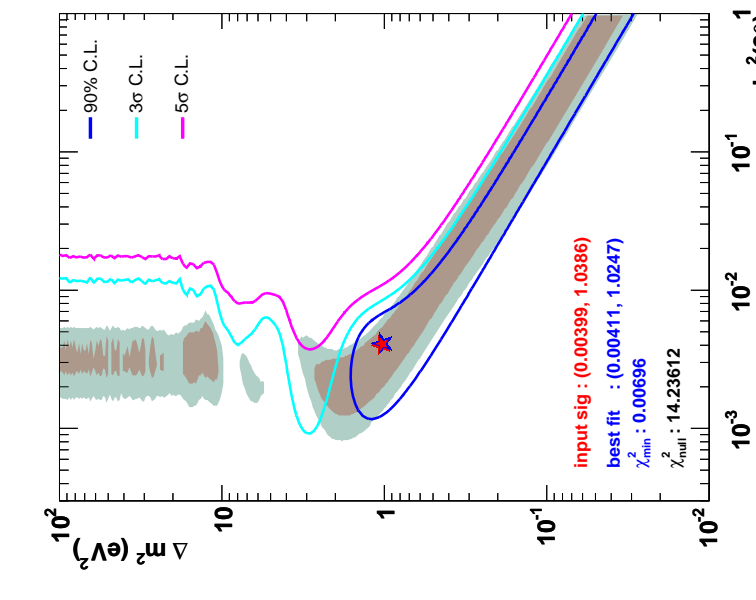

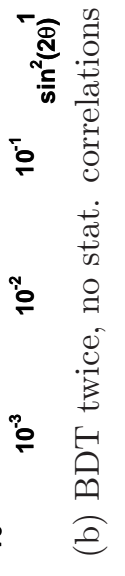

.

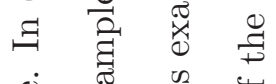

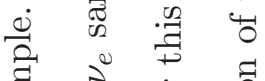

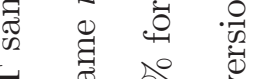

1 $\begin{array}{lll}\pi & 0 & 0 \\ 0 & 0 & 0\end{array}$

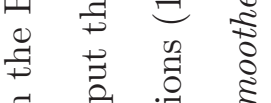

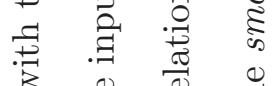

范

$\vec{n}=0.7$

क 1 .

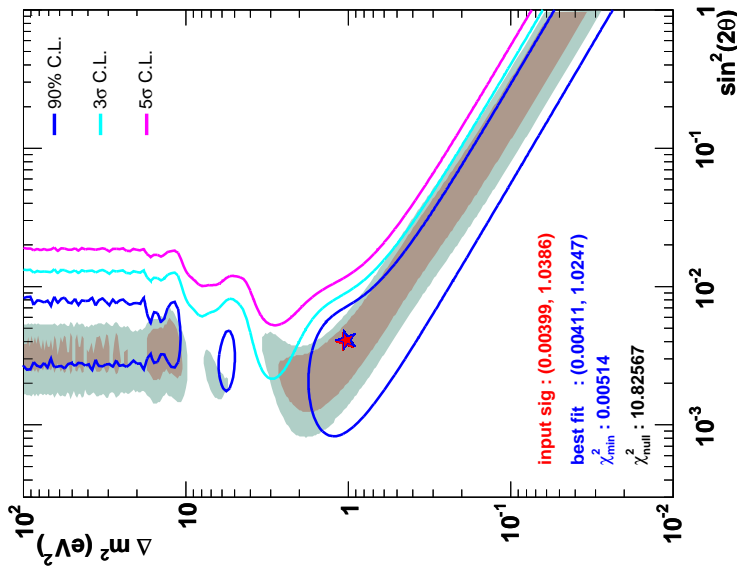

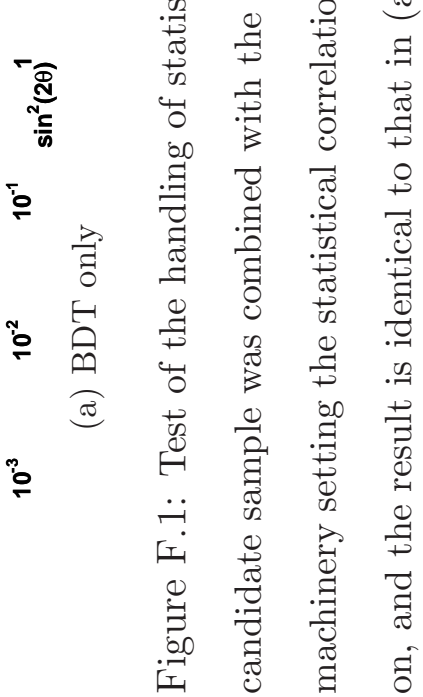




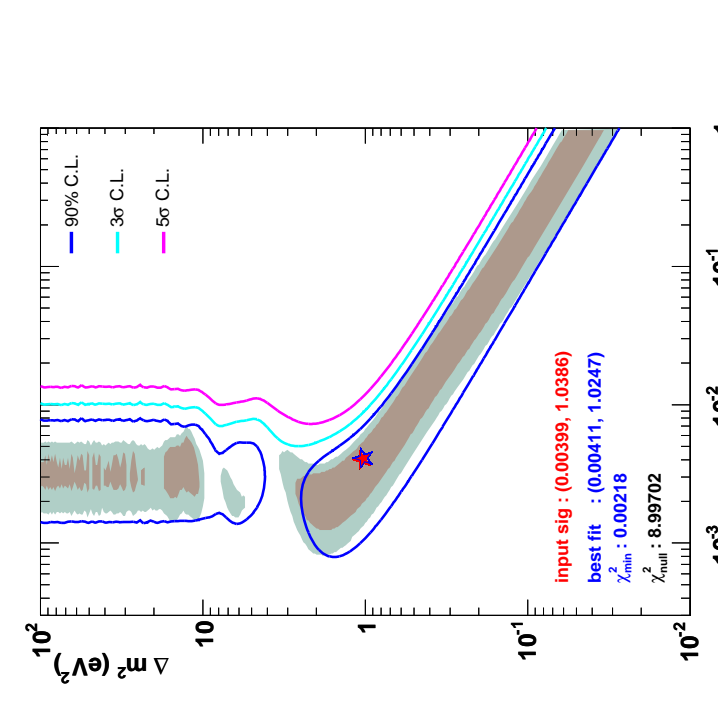

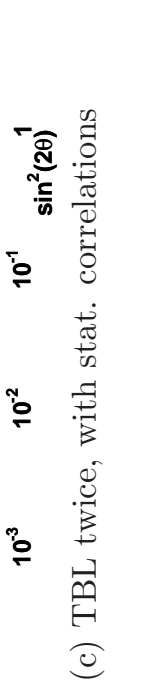

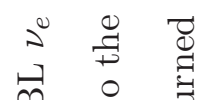

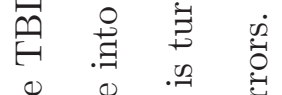

๙

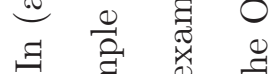

0.

द्वे गे

क छ्ञ

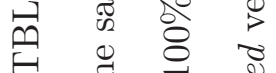

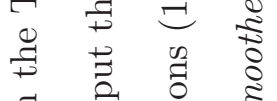

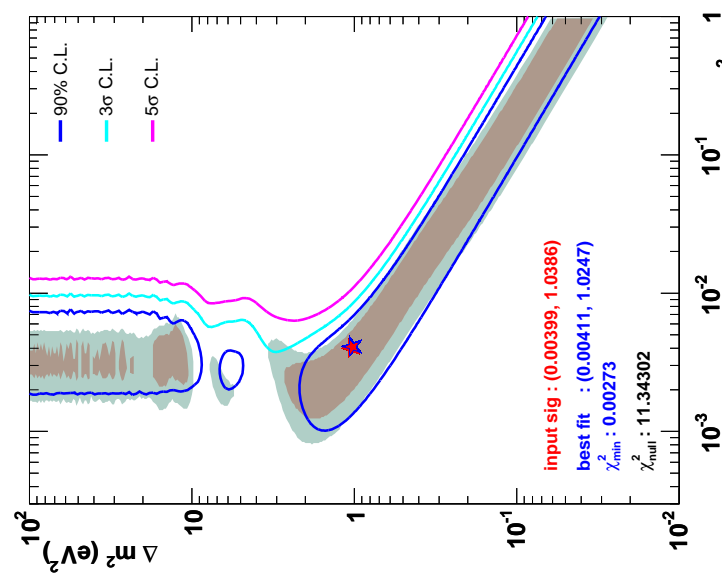

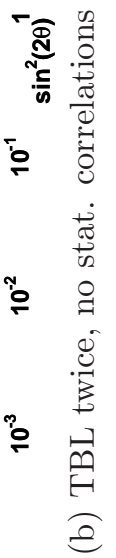

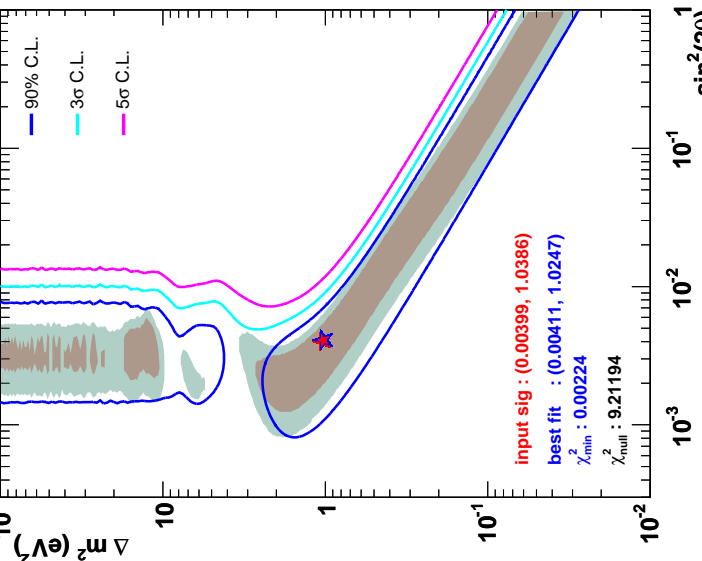

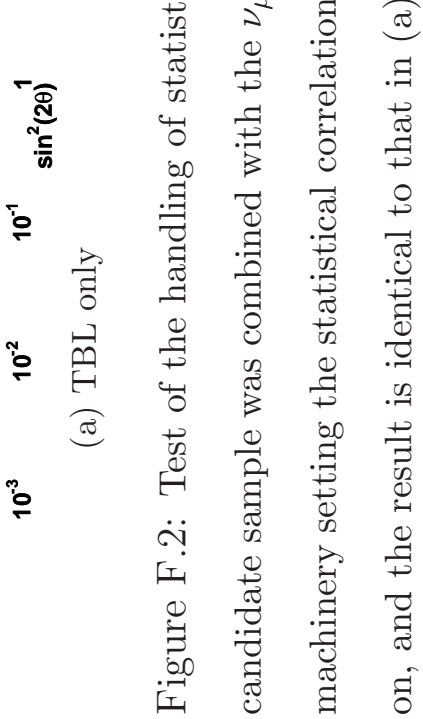




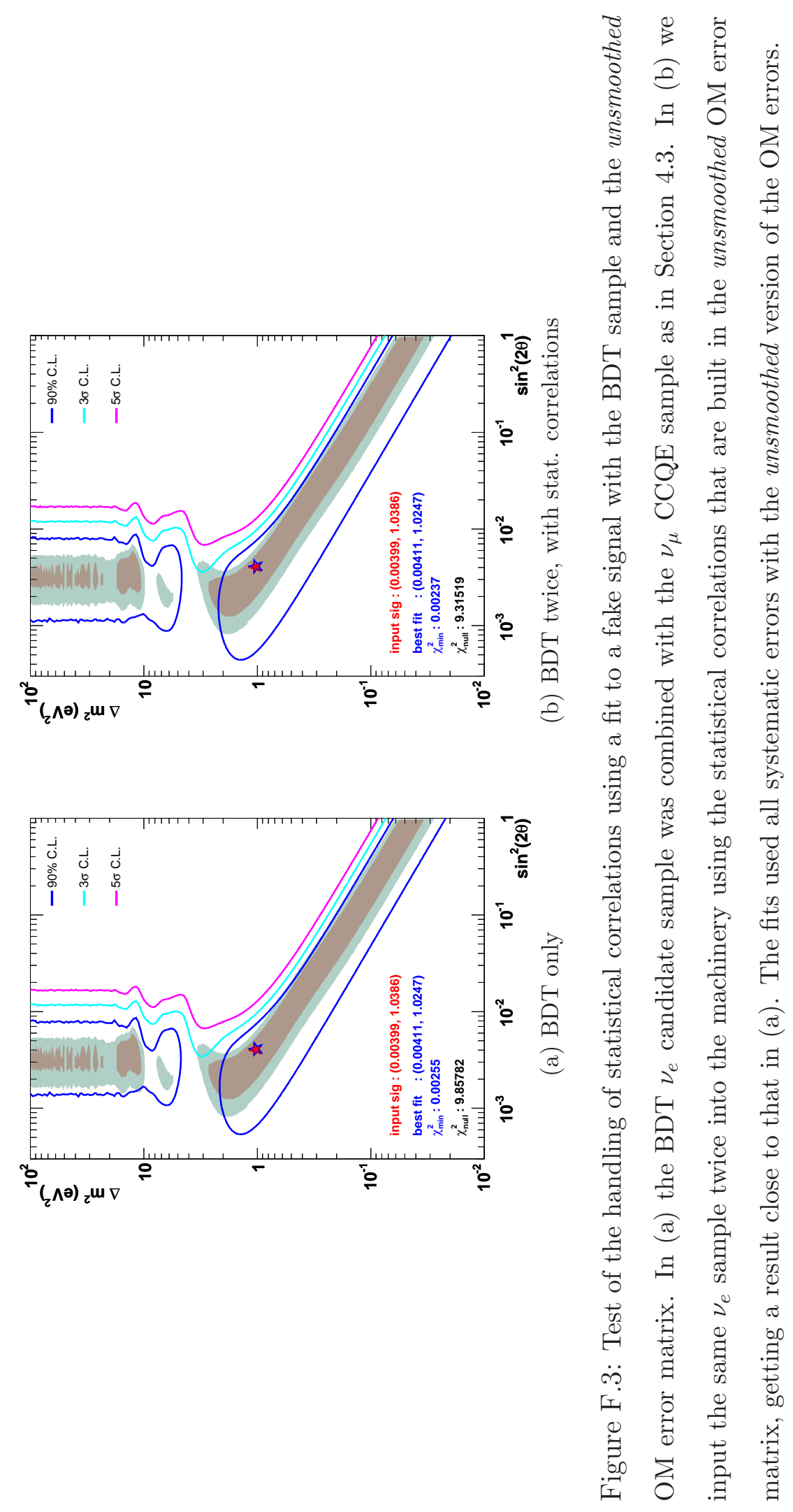




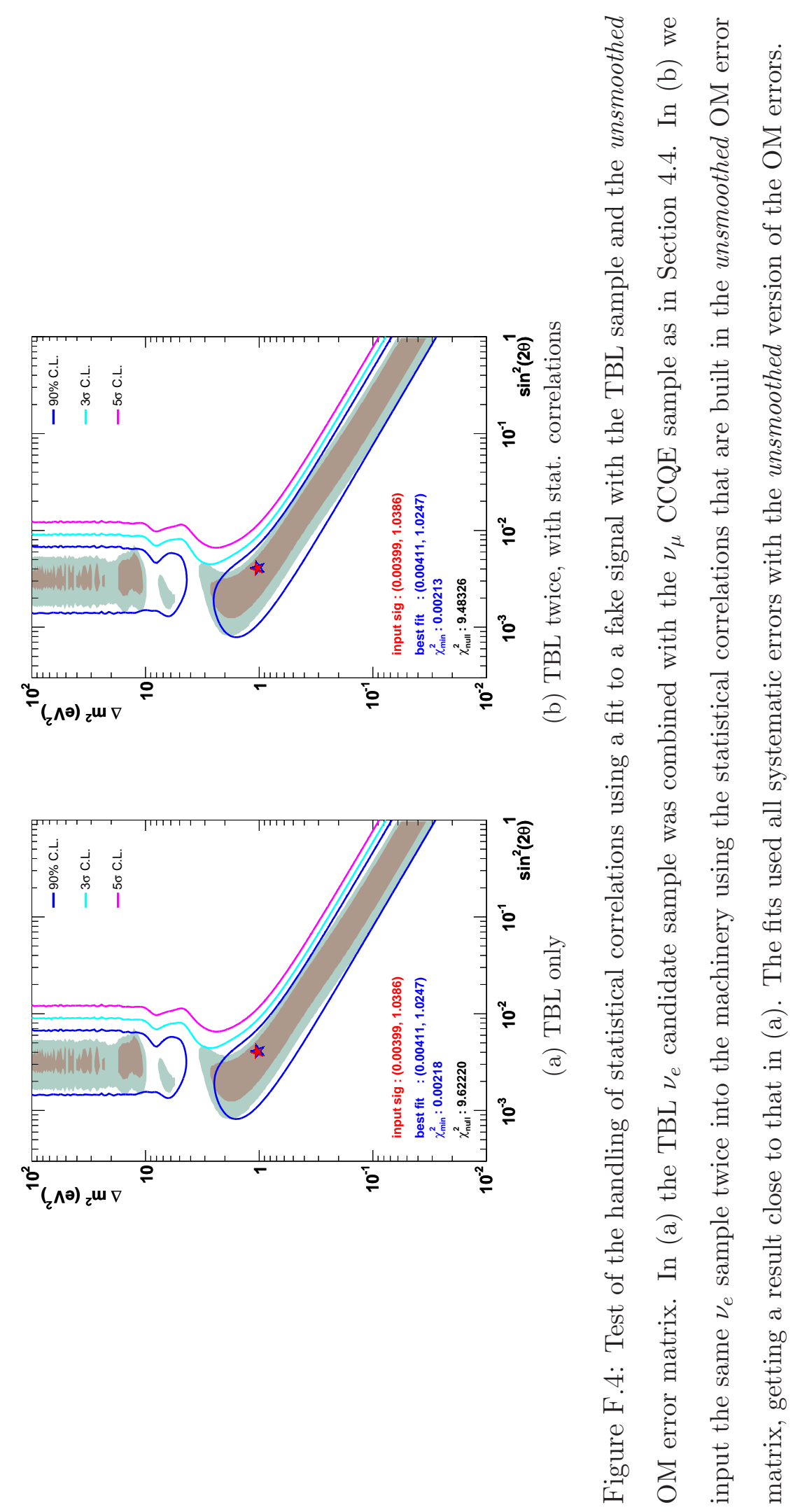




\section{F.2 Slices of the $\chi^{2}$ surface}

Slices of the $\Delta \chi^{2}$ surface defined in Section 4.2 .3 for a sensitivity calculation are shown in Figure F.5 for various values of $\Delta m^{2}$. The minimum $\sin ^{2} 2 \theta$ found for all $\Delta m^{2}$ values is by definition equal to zero for a sensitivity. The horizontal line at $\Delta \chi^{2}=1.64$ intersects the three curves (BDT in black, TBL in red, and BDT/TBL combined in green) at the $\sin ^{2} 2 \theta$ value where the $90 \%$ C.L. sensitivity curves of Figure 5.1(a) lie.

The corresponding comparison for the fits to the open data are shown in Figure F.6. In this case the fit finds a very small signal which is consistent with zero within the uncertainties. Again, the intercepts of the constant at $\Delta \chi^{2}=1.64$ with the curves correspond to the $\sin ^{2} 2 \theta$ values of the $90 \%$ C.L. limits calculated in Figure 5.3(b)

In both the sensitivity and limits, the addition of the two $\nu_{e}$ candidate samples makes the $\Delta \chi^{2}$ surface more steep as a function of $\sin ^{2} 2 \theta$, which causes the C.L. contours to become narrower around the minimum, which is always near $\sin ^{2} 2 \theta=0$.

\section{F.3 Example fits to fake data sets}

In this section we present the result of fits to fake data sets with a toy set of PID selection cuts. Except for the results shown in Figure F.9 and Figure F.10, the studies below do not correspond to the main results presented in this thesis and are shown only to demonstrate the workings of the CombinedFit package machinery.

\section{F.3.1 Constructing fake datasets}

Fake datasets are used to study the fitting procedure and to determine its statistical capabilities and shortcomings for addressing neutrino oscillations of the LSND type. 

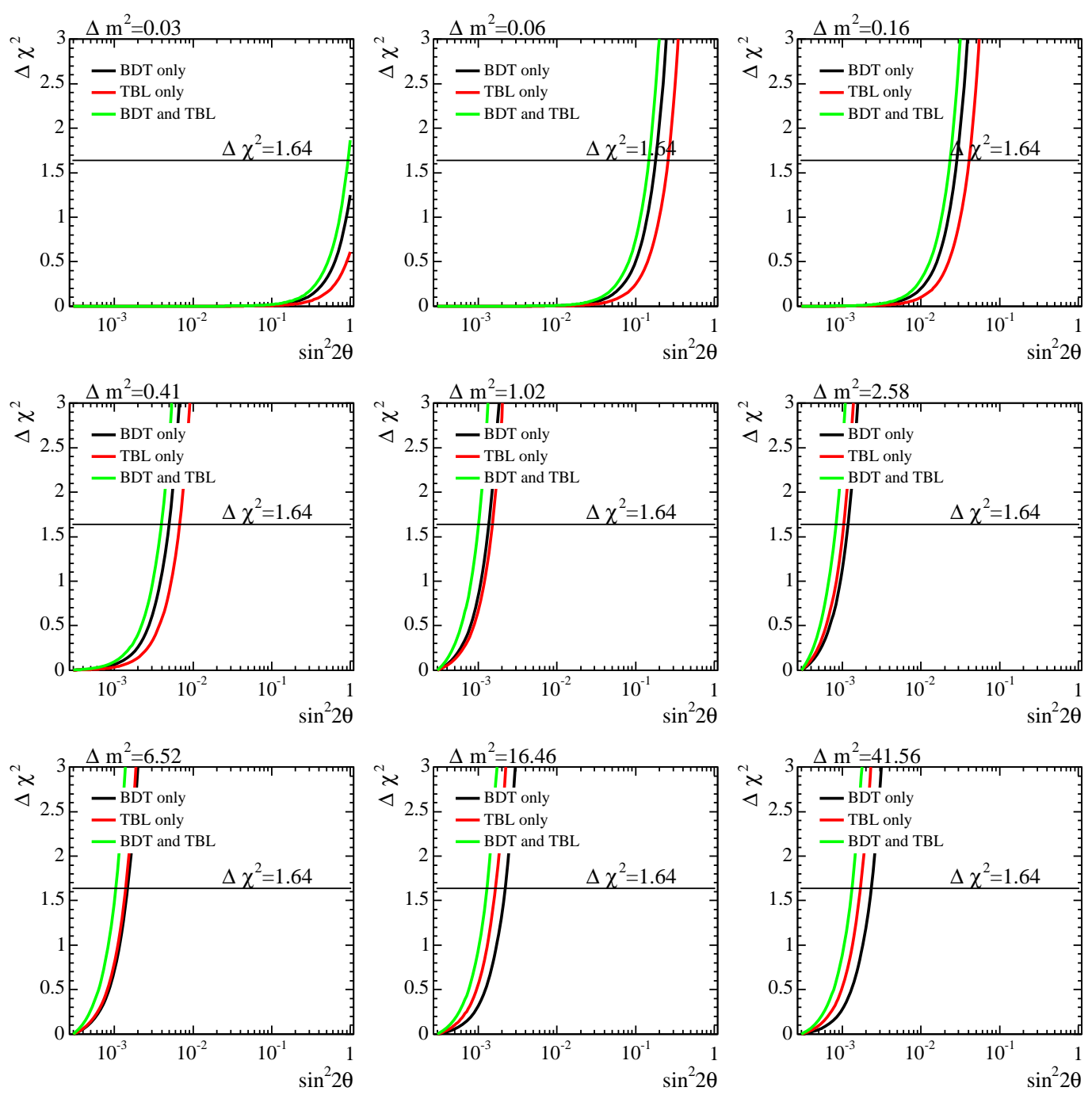

Figure F.5: Slices of the raster-scan $\Delta \chi^{2}$ surface defined in Section 4.2.3 for the sensitivity calculation using either one or both of the $\nu_{e}$ candidate samples combined with the $\nu_{\mu}$ sample. 

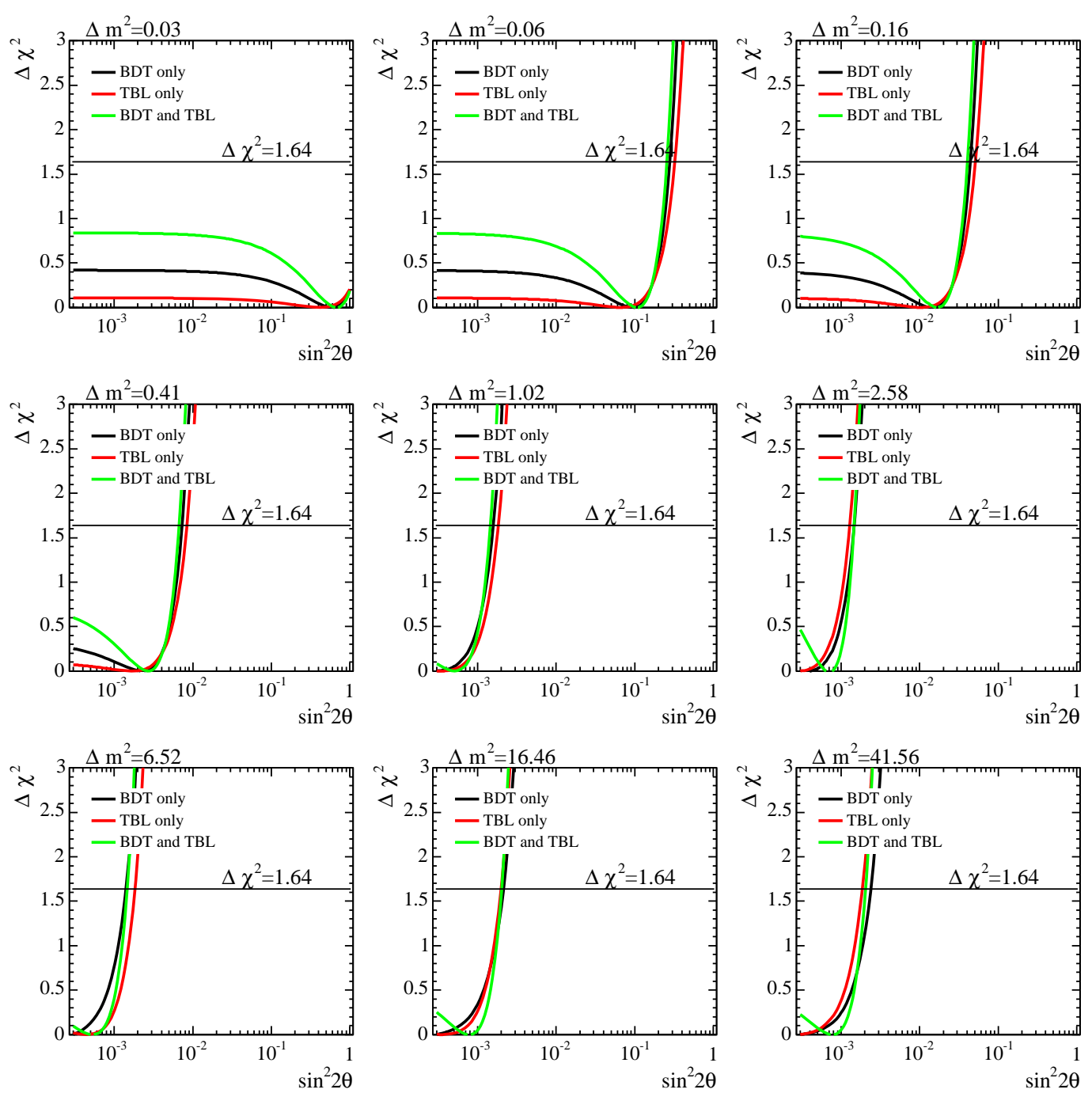

Figure F.6: Slices of the raster-scan $\Delta \chi^{2}$ surface defined in Section 4.2 .3 for the fits to the open data using either one or both of the $\nu_{e}$ candidate samples combined with the $\nu_{\mu}$ sample. 
Starting from the Monte Carlo prediction for the $\nu_{\mu}$-CCQE and $\nu_{e}$ distributions after the selection cuts have been applied, one can generate fluctuated distributions that are consistent with statistical and/or systematic errors.

\section{Statistical fluctuations}

If $n$ is the number of events in a given bin of the $E_{\nu}^{Q E}$ distribution is, a new number of events $x$ is randomly thrown from the Poisson probability distribution

$$
p(x, n)=\frac{e^{-n} n^{x}}{x !}, \quad x=0,1,2, \ldots,
$$

the new bin contents $x$ replaces the original number and the procedure is repeated for all the bins in both the $\nu_{\mu}$-CCQE and $\nu_{e}$ distributions. The fluctuated event numbers are all independent and the size of the fluctuation is determined by the statistical error $\sqrt{n}$ in the contents of each bin.

\section{Systematic fluctuations}

These are generated using the method of the lower triangular factorization of the error matrix described in [162]. In this method, a random $n$-dimensional column vector $\mathbf{Y}$ belonging to a population described by the multivariate Gaussian distribution with $n \times n$ covariance matrix $\mathbf{M}$ and mean $\langle\mathbf{Y}\rangle=\mathbf{0}$ is generated as follows:

1. Find a lower triangular matrix $\mathbf{H}$ such that $\mathbf{M}=\mathbf{H} \mathbf{H}^{T}$

2. Generate $n$ independent random numbers $X_{1}, \ldots X_{n}$ with zero mean and unit variance

\section{Return $\mathbf{Y}=\mathbf{H X}$}

If $\mathbf{N}$ is a $n$-dimensional array containing the number of events in a given distribution and the matrix $\mathbf{M}(n \times n)$ is the error matrix describing the correlations between 
the contents if the various bins of $\mathbf{N}$, then one can form the fluctuated distribution $\mathbf{N}^{\prime}$ as:

$$
\mathbf{N}^{\prime}=\mathbf{N}+\mathbf{Y}
$$

The distribution described by $\mathbf{N}^{\prime}$ will be a fluctuation of $\mathbf{N}$ consistent with the covariance matrix $\mathbf{M}$.

\section{Example of fits to fake data with strong/null LSND-like signals}

When an oscillations signal is present, signal and background $\nu_{e}$ events determine the total contents of each bin of the $\nu_{e}$ distribution, and the sum must be used to form a consistent statistical fluctuation. Note that statistical fluctuations of the $\nu_{\mu}$-CCQE dataset are very small due to the large numbers of events in that sample.

The result of the fit over a fake dataset with a strong (absent) signal using the $\mathrm{BDT}+\nu_{\mu}$ fit is shown in Figure F.7 (Figure F.8). We can compare the fake data distributions with the original Monte Carlo prediction in the stacked plot of Figure F.7(a) (Figure F.8(a)), which also shows the composition of the samples. The large error bars shown are the total errors as directly extracted from the multisim procedure described in Section 4.2.2. The second panel Figure F.7(b) (Figure F.8(b)) shows the effect that the high statistics $\nu_{\mu}$ sample has on the distributions when it is allowed to constrain the Monte Carlo predictions. In this case the $\nu_{\mu}$-CCQE distribution is made to be statistically identical to the fake $\nu_{\mu}$-CCQE distribution, and this reshaping and normalization affects the $\nu_{e}$-CCQE distribution by means of the terms connecting the bins of the two samples in the total error matrix. The error bars on the $\nu_{e}$ sample have also been reduced by the $\nu_{\mu}$-CCQE constraint. The third panel Figure F.7(c) (Figure F.8(c)) shows the fit result in terms of the event excess over the expected backgrounds and compares it with two sample LSND solutions at low 
and high values of $\Delta m^{2}$. In this plot the data points have total errors. Finally, the fourth panel, Figure F.7(d) (Figure F.8(d)) shows the fit result in terms of allowed regions in the oscillations parameter space for various levels of confidence.

It is important at this stage to notice that the result of the fit to the fake dataset with no signal produced a best fit point that is distant from the exactly zero-signal solution, however, the size of the total uncertainties is such that this best fit point is consistent with it. The particular point that is chosen by the fit is in the $\sim$ high $\Delta m^{2}$ region. This behavior is typical of fits to very small signals and it is due to finding a minimum $\chi^{2}$ when some of the statistical fluctuations near the tail of the data distribution can be mimicked by an oscillatory behavior typical of a large $\Delta m^{2}$. Given the size of the uncertainties, signals that are fit in this region of the space are indistinguishable from zero-signal solutions.

Examples of fits to fake signals with the extended machinery used for the result in this thesis are also shown in Figure F.9 for a strong LSND-like signal, and in Figure F.10. The fit in Figure F.9 provides a good example of how the fit takes care of an overall normalization fluctuation in the $\nu_{\mu}$ CCQE sample and correctly finds the true signal after this normalization has been propagated to the $\nu_{e}$ predictions through the $\nu_{\mu}-\nu_{e}$ correlations in the error matrix as described in Section 4.2.4. The fit in Figure F.9 shows a similar effect. The fitted signal in this case is indistinguishable from the true negligible signal. 

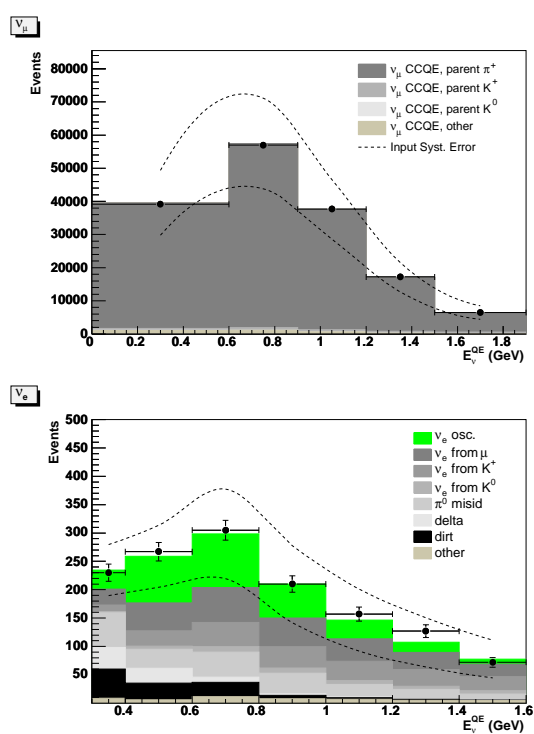

(a) Before fit

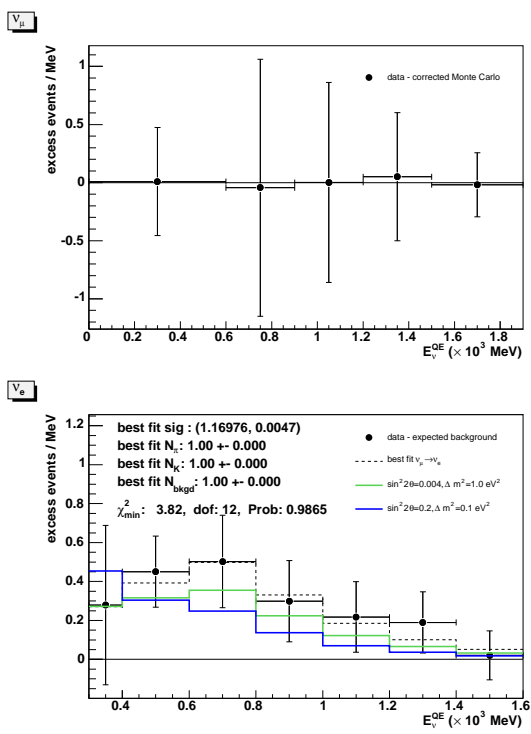

(c) Fit result (event excess)
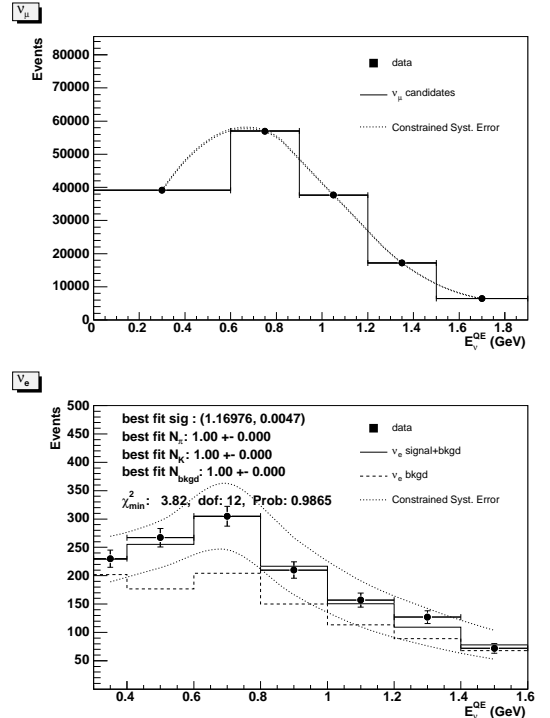

(b) Fit result

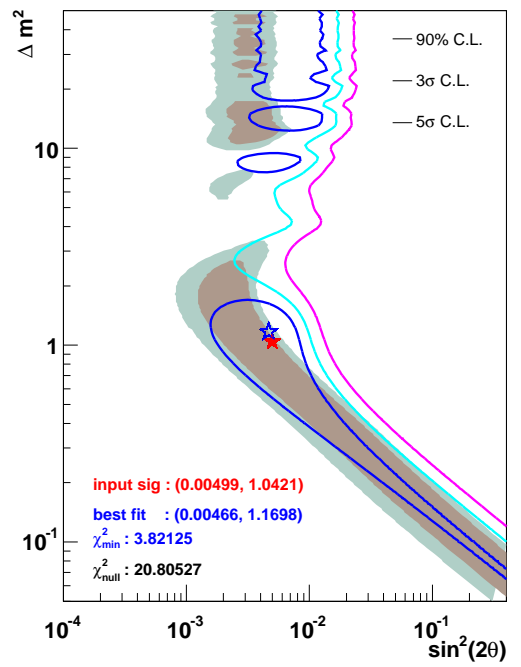

(d) Allowed regions

Figure F.7: Fitting a fake data set with a strong signal. In (a) the green component is the true oscillations signal, and the dotted curves represent the unconstrained systematic uncertainties. In (b) the dotted curves represent the constrained systematic uncertainties calculated as described in Section 4.2.4. In (d) the red star indicates the true signal and the blue star indicates the best fit. 

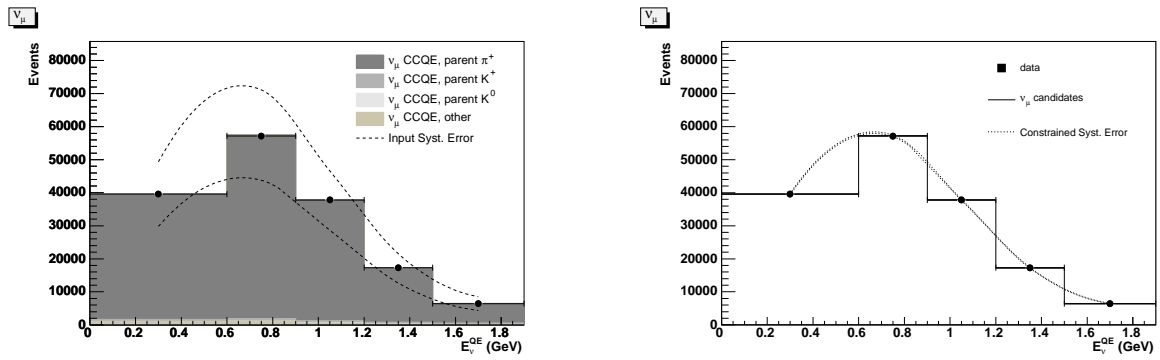

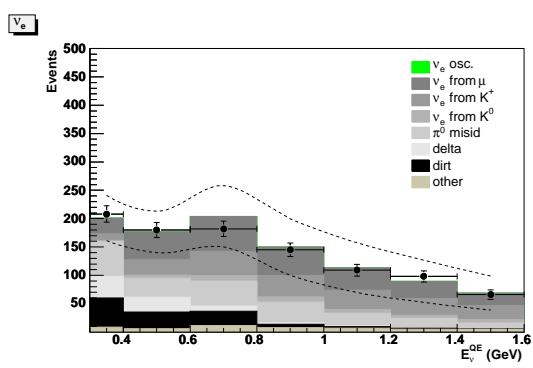

(a) Before fit

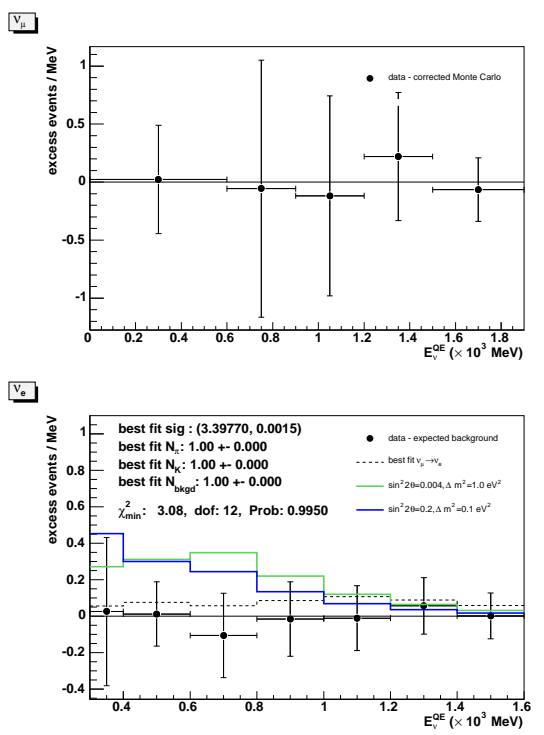

(c) Fit result (event excess) $v_{e}$

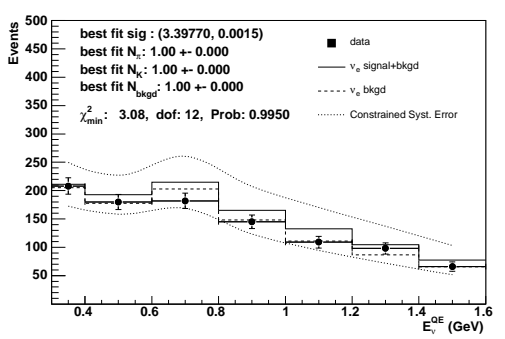

(b) Fit result

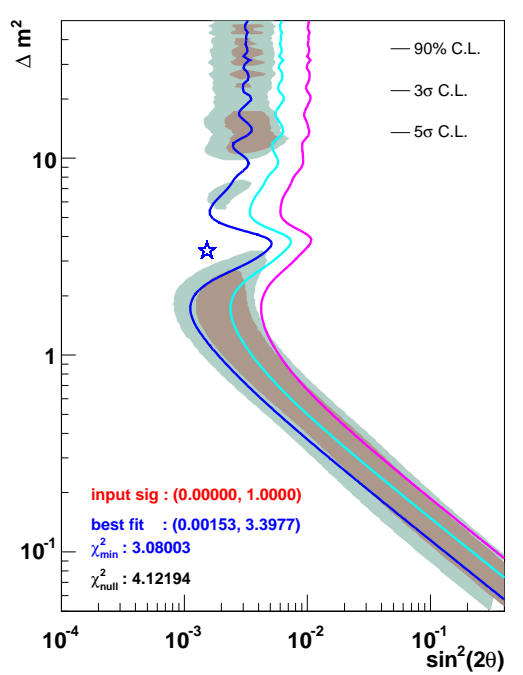

(d) Allowed regions

Figure F.8: Fitting a fake data set with no oscillations signal. In (a) the green component is the true oscillations signal, and the dotted curves represent the unconstrained systematic uncertainties. In (b) the dotted curves represent the constrained systematic uncertainties calculated as described in Section 4.2.4. In (d) the blue star indicates the best fit. 


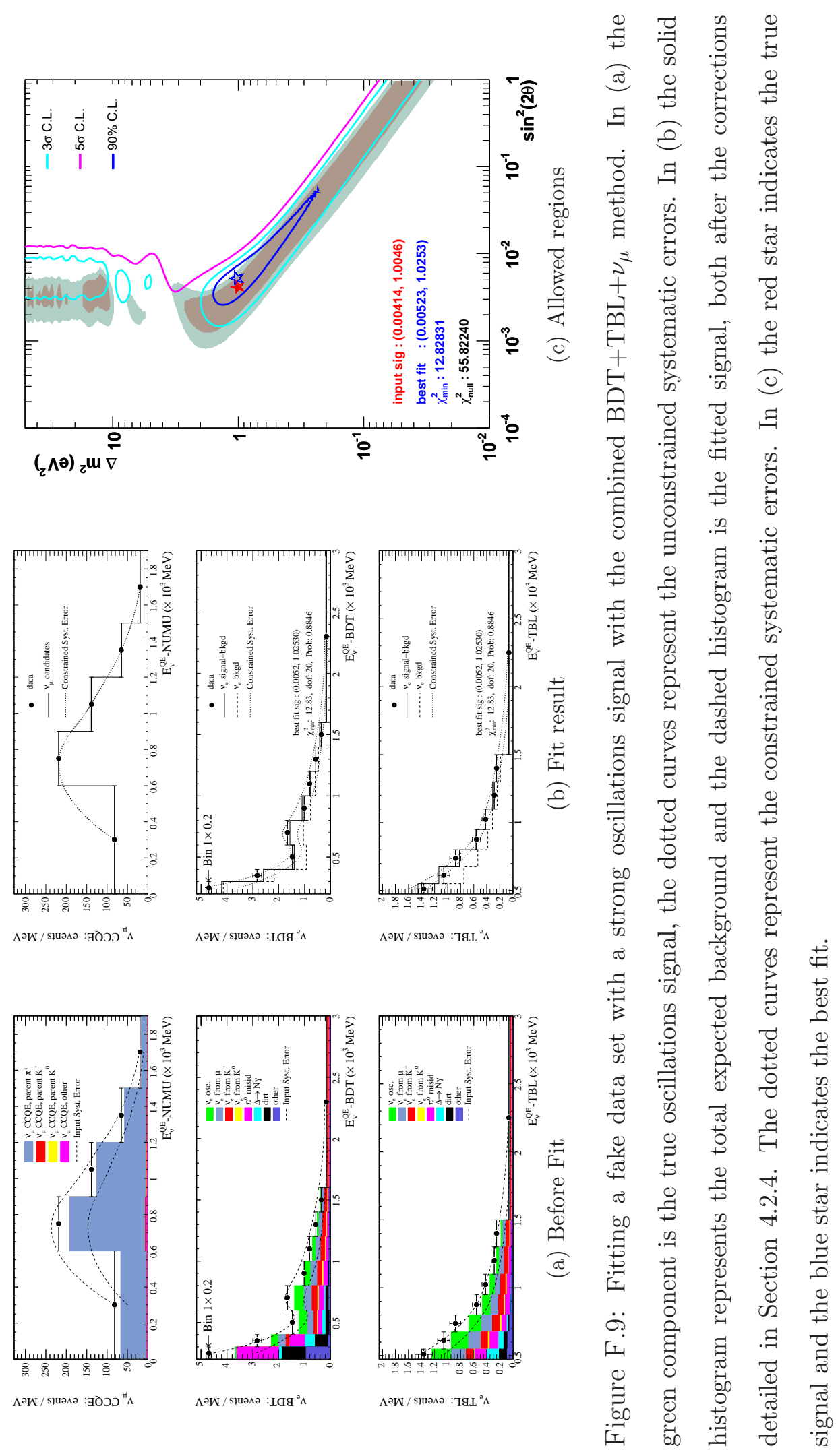




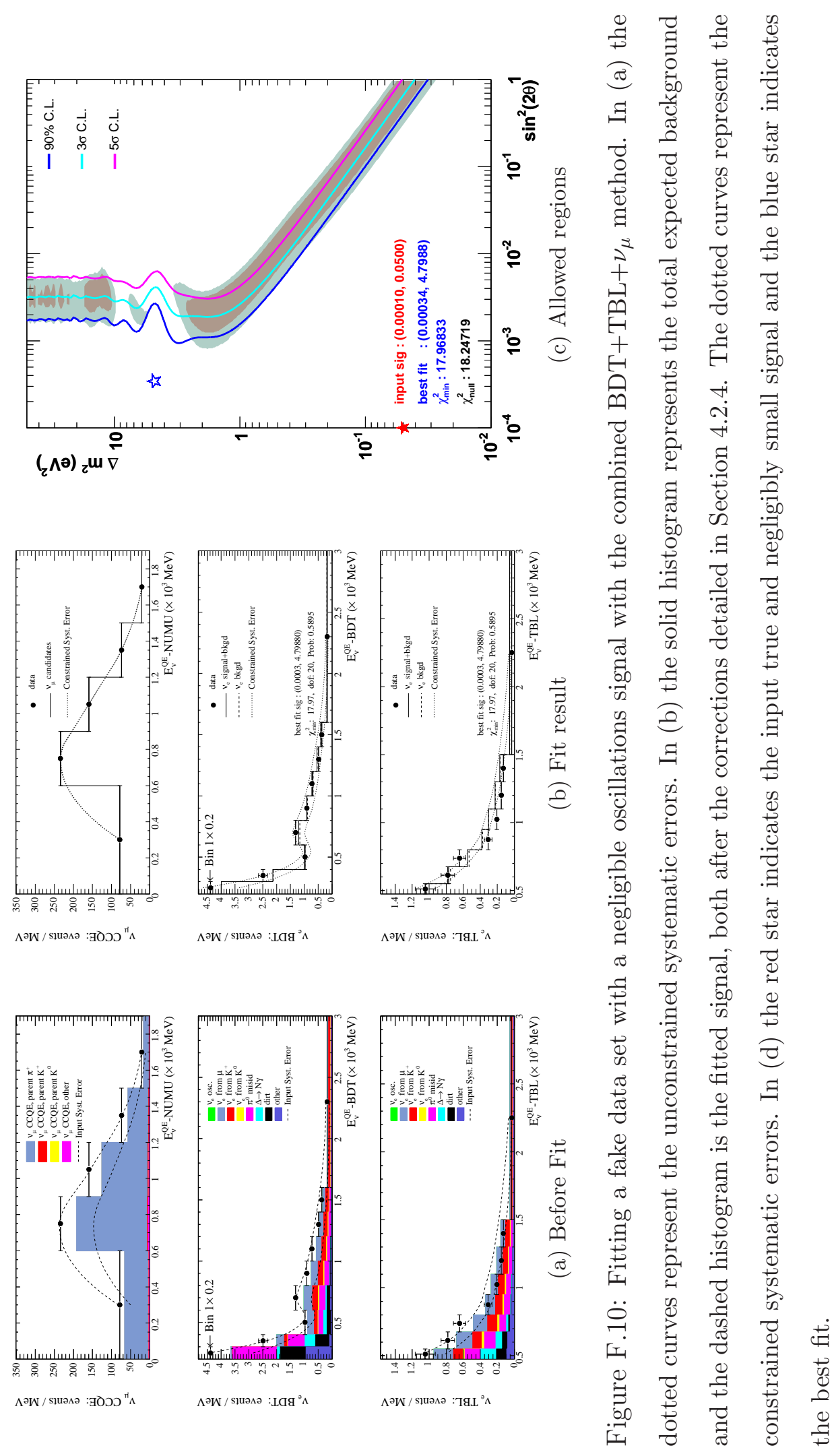




\section{Appendix G}

\section{Events from the NuMI Beamline}

The NuMI beamline in Fermilab delivers an intense neutrino beam to the MINOS [84] experiment. The neutrinos are produced by the interaction of $120 \mathrm{GeV}$ protons from the Fermilab Main Injector (MI) onto a 2 interaction length graphite target. Secondary particles are focused by two magnetic horns whose configuration can be adjusted to modify the energy spectrum of the neutrinos. The focused secondaries decay along a $\sim 600 \mathrm{~m}$ long decay tunnel which points towards the MINOS near detector located at about $740 \mathrm{~m}$ from the target. MiniBooNE subtends an angle of 111 mrad from the face of the first horn.

\section{G.1 The NuMI Trigger in MiniBooNE}

The signal to extract the proton beam from the MI to the NuMI beamline is called "Main Injector Beam Synchronization event (MIBS) \$74". The MIBS clock broadcasts a $\$ 74$ signal approximately every two seconds during normal operation conditions. This signal has a fixed time delay relative to the actual physical firing of the 
extraction magnets that take the beam from the MI into the NuMI beamline ${ }^{1}$.

Event $\$ \mathbf{7 4}$ originates at the location MI-60 (see Figure G.1) on the MI accelerator, which houses the NuMI kicker extraction magnets. A copy of $\$ 74$ is branched out to the Main Accelerator Controls (MAC) room where it triggers several reflected signals (called TCLK events), among which is the \$A9 signal that eventually reaches the MiniBooNE experimental hall and is used as trigger. TCLK events originate in the MAC room and are distributed over the accelerator complex trough utility tunnels and duct banks. The TCLK \$A9 follows a branch heading directly back to MI-60, where the original $\mathbf{\$ 7 4}$ event was produced. Once $\mathbf{\$} \mathbf{A} \mathbf{9}$ reaches MI-60, it is sent to the MI ring locations MI-60 North, MI-62, MI-65, MI-8, MI-10, MI-12, and finally to the MiniBooNE detector building following the path shown in Figure G.1. The time delay between MIBS $\$ 74$ and TCLK $\$$ A9 at MI-60 was measured to be $14.5 \pm 1 \mu \mathrm{s}^{2}$. This time is the sum of travel times of the $\$ \mathbf{7 4}$ from MI-60 to the MAC room $(\sim 6$ $\mu \mathrm{s})$ plus $\sim 2.8 \pm 1 \mu \mathrm{s}$ hardware decode/encode delay in the MAC room plus the travel time of the $\$ \mathbf{A 9}$ back out to MI-60 $(\sim 6 \mu \mathrm{s})$.

At the end of this $14.5 \mu$ s period after the $\$ 74$ was originated, the TCLK $\$$ A9 has just arrived at MI-60 on its way to MINOS (and the other locations in between), the $\$ 74$ is $14.5 \mu$ s downstream of it en route to the same points, and the timer on the NuMI kicker has counted down from $222.0005 \mu$ s to $207.5 \mu$ s. The $\$$ A9 starts its travel along the fiber optics path towards MINOS following the $\$ \mathbf{7 4}$, and will take $\sim 8 \mu$ s to get to the MiniBooNE vault.

The \$A9 signal is extracted from the Internet Relay Monitor (IRM) at the MiniBooNE detector vault adding a $\sim 1.6 \mu$ s decode time to its delay (the signal is taken from IRM channel 8). Taking into account a beam time of flight of $\sim 1.5 \mu \mathrm{s}$, the time

\footnotetext{
${ }^{1}$ This delay is set to $20.04932 \mathrm{MI}$ revolutions, with $1 \mathrm{MI}=11.07272 \mu \mathrm{s}$, this is $222.00050 \mu \mathrm{s}$.

${ }^{2}$ Measured by Greg Vogel, FNAL Accelerator Controls. The uncertainty is dominated by a $\sim 1$ $\mu \mathrm{s}$ encoding/decoding time jitter.
} 


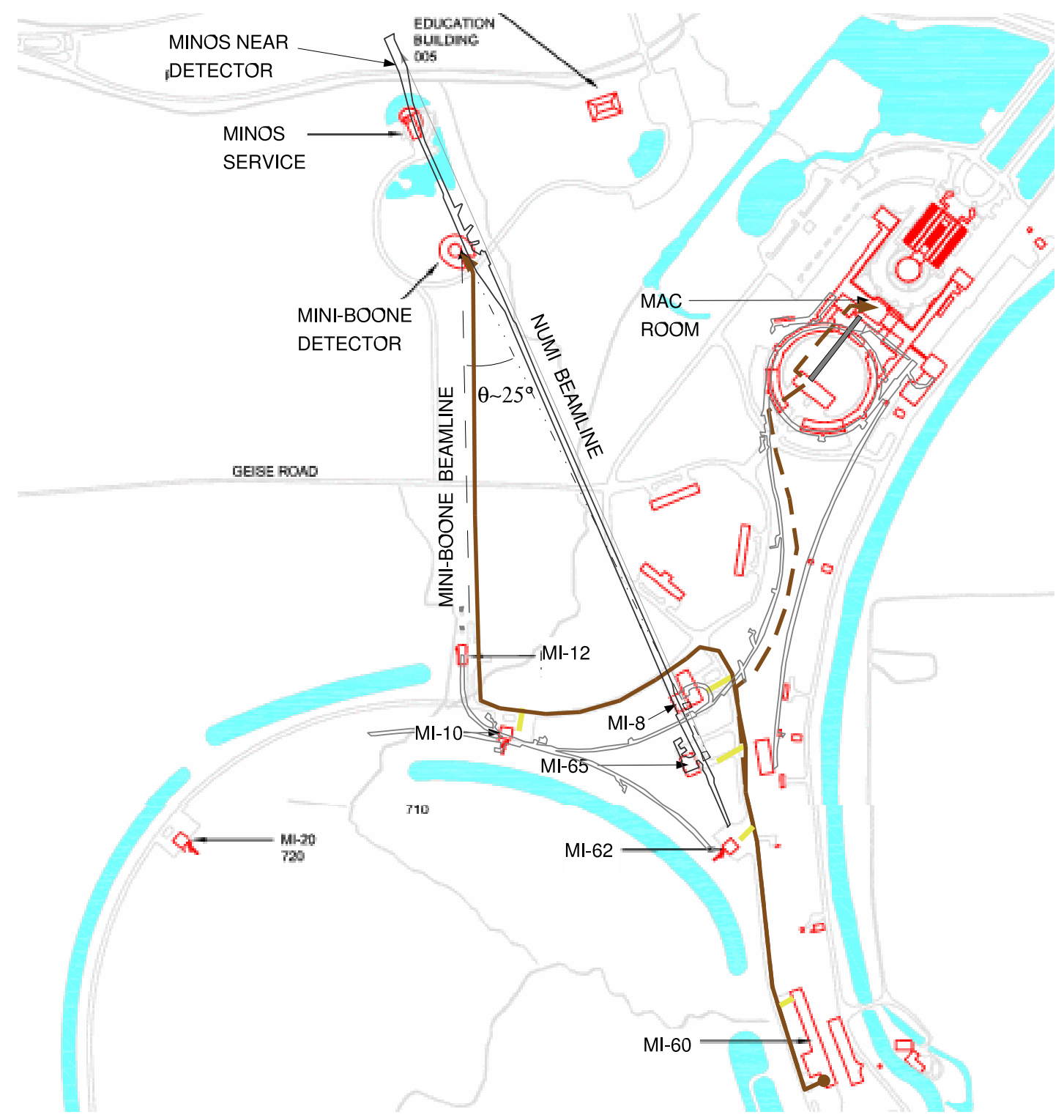

Figure G.1: Path of trigger signal along communications ducts. The MIBS \$74 event originates at MI-60 and travels along the dark solid path until MI-8 from where it continues along the dashed path to the MAC room (X-Gallery). A reflected TCLK event (\$A9) is sent back from MAC to MI-60 along the same path, and then follows the full length of the path marked by the solid line towards the MiniBooNE detector enclosure, where it is read out from an IRM and input into the MiniBooNE trigger crate. 
Table G.1: Relevant times for the determination of the time delay of the NuMI event trigger in MiniBooNE. The total delay shown is the setting of the ACNET parameter E:MBDT3. Only the dominant uncertainties are shown.

\begin{tabular}{lcr}
\hline Description & \pm & Time \\
\hline Delay between $\$ 74$ and kickers firing & $(+)$ & $222.0005 \mu \mathrm{s}$ \\
Beam time of flight from kickers to NuMI target & $(+)$ & $1.5 \mu \mathrm{s}$ \\
Delay between $\$ 74$ and $\$$ A9 arriving at MI-60 & $(-)$ & $14.5 \pm 1 \mu \mathrm{s}$ \\
\$A9 fiber optics delay from MI-60 to MB vault & $(-)$ & $\sim 7 \mu \mathrm{s}$ \\
Decode time of \$A9 at IRM in MB vault & $(-)$ & $\sim 0.6 \pm 0.5 \mu \mathrm{s}$ \\
Early opening of DAQ window (catch rising edge) & $(-)$ & $2 \mu \mathrm{s}$ \\
\hline Total delay & & $199.4 \pm 1.1 \mu \mathrm{s}$ \\
\hline
\end{tabular}

lapse between the decoding of the $\mathbf{\$ A 9}$ at the MiniBooNE vault and the beam hitting the tank was estimated to be $201.4 \pm 1 \mu \mathrm{s}$. An additional delay of $2 \mu \mathrm{s}$ was added in order to open the $19.2 \mu$ s window approximately $2 \mu$ s before the beam goes through. The relevant times used to decide the delay of the IRM signal \$A9 to be input to the trigger system are shown in Table G.1, where the final delay setting of $199.4 \pm 1.1$ $\mu \mathrm{s}$ is shown in the last row. The uncertainty of $\sim 1 \mu \mathrm{s}$ will smear the $1.6 \mu \mathrm{s}$ batch structure of the NuMI beam, as is shown in Figure G.2.

The NuMI trigger signal is input as an OR with the Debuncher, ZeroBias and Strobe triggers into the E2 bit of the DAQ electronics rack \#13. It is distinguished from the other E2 inputs by setting its pulse width to $750 \mu \mathrm{s}$, meaning that either 7 or 8 DAQ clock ticks will be asserted during this time interval after a $\$ \mathbf{A} 9$ is received. When a NuMI event trigger has been identified a look-back into the circular buffer is performed opening a window of $19.2 \mu \mathrm{s}$. 


\section{Observing NuMI events}

NuMI triggers are stored in the BigNu data stream in the MiniBooNE DAQ system. They are distinguished from other triggers also stored in that stream by being assigned the trigger event number \#19. Typically, a filter to select this trigger event number and a latency filter are applied to the BigNu stream to form NuMI event data sets suitable for subsequent analysis.

Events in the tank are easily observed by applying the usual neutrino candidate cuts on the first sub-event: THits $[0]>200$ and VHits $[0]<6$. As shown in Figure G.2 the neutrino events can be clearly seen in the NuMI beam window of $\sim 10 \mu$ s even before any cuts. The 5-6 batch structure of the NuMI beam is visible, although it is greatly smeared by the jitter in the triggering signal coming from the encoding and decoding time variations that affect the MIBS $\$ 74$ and $\$$ A9 accelerator signals described earlier. This smearing effect prevents the detector from resolving the fine timing structure of the spills.

Good proton spills from the beam are selected using the beam quality cuts detailed in Table G.2. Beam spill information was obtained from MINOS collaborators [103] in the form of beam summary ntuples containing the beam position and spread at the target, the horn current and the target position defining the beam configuration, and the UTC time stamp of each spill since the beginning of the run in May 20th 2005. The majority of the data acquired with the NuMI event trigger corresponds to the so called Low Energy-10 (LE10) configuration of the NuMI beam, which is tuned to have a softer energy spectrum at the MINOS far detector than other possible configurations. Data taken in the LE10 configuration was used in the first oscillations results from the MINOS experiment [87][88]. The defining characteristics of this beam configuration are a current flow across the two-horn system of -175 kA and a target displacement of $10 \mathrm{~cm}$ upstream of the nominal target position. Other configurations 


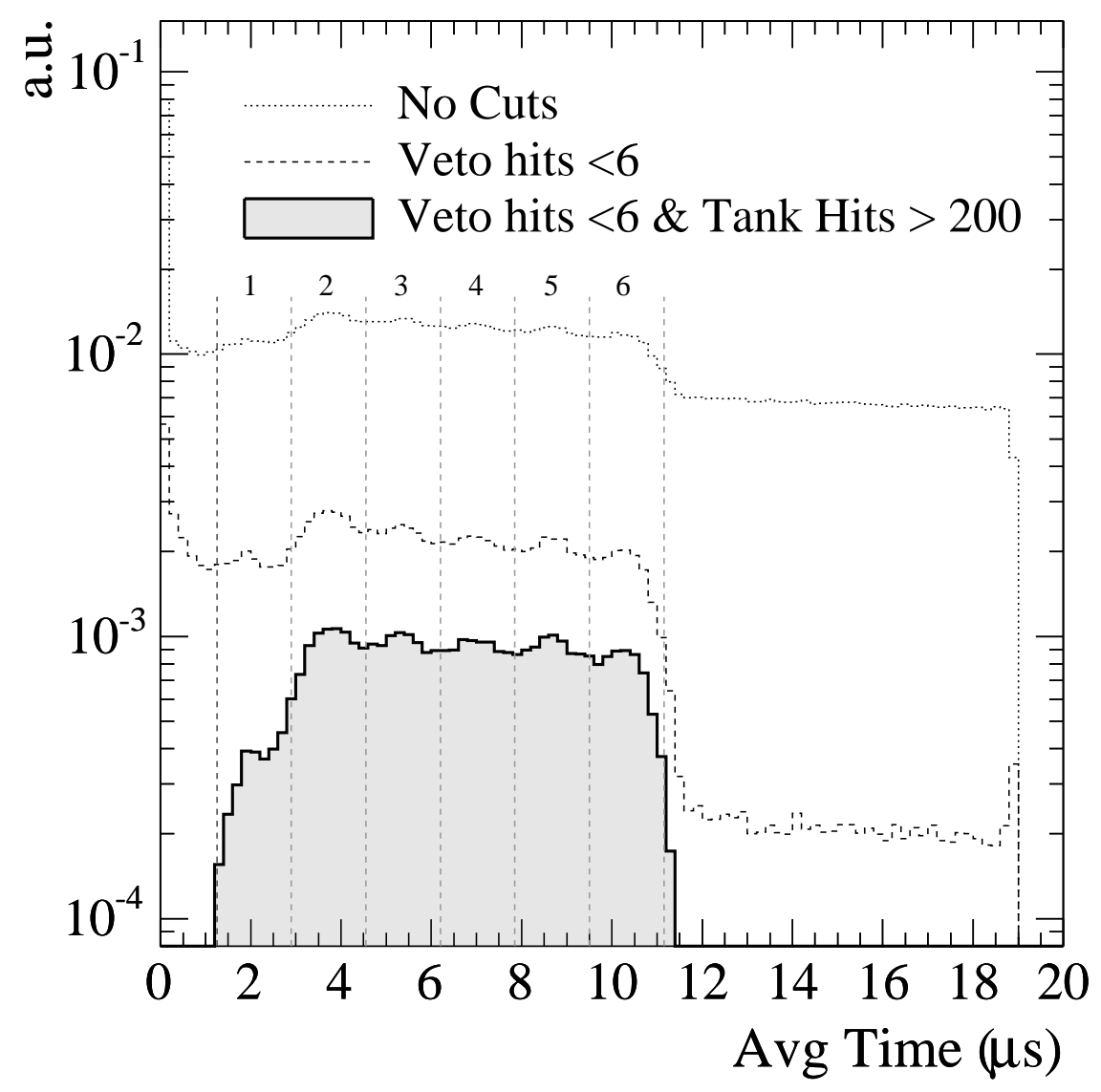

Figure G.2: Time distribution of neutrino candidates from the NuMI beam in the MiniBooNE DAQ window. The events are required to satisfy: no cuts (dotted line), negligible veto activity in the first sub-event (VHits $[0]<6$, dashed line), negligible veto activity and more than 200 tank PMT's in the first sub-event (VHits[0]<6 \& THits[0]>6, solid filled). The vertical lines indicate the 6-batch structure of the NuMI beam. The earliest batch is extracted from the Main Injector only $\sim 40 \%$ of the time. 
of the beam have been exercised by the NuMI-MINOS collaborators in order to study the systematics of the beam simulation. A detailed study of the NuMI beam and its systematics can be found elsewhere [82].

Table G.2: Quality cuts applied to select good spills from the NuMI beam. The selection of the horn current corresponds to the LE10 configuration of the NuMI beamline used for the first result from the MINOS experiment [87].

\begin{tabular}{lcl}
\hline \multicolumn{2}{c}{ NuMI beam quality cuts } & units \\
\hline \hline Beam Spill Intensity $\left(I_{B}\right)$ & $I_{B}>0.1 \times 10^{12}$ & protons/spill \\
Horizontal Beam Position at target $\left(X_{B}\right)$ & $-2.0<X_{B}<0.0$ & $\mathrm{~mm}$ \\
Vertical Beam Position at target $\left(Y_{B}\right)$ & $0<Y_{B}<2.0$ & $\mathrm{~mm}$ \\
Horizontal Beam Width at target $\left(\Delta X_{B}\right)$ & $<1.5$ & $\mathrm{~mm}$ \\
Vertical Beam Width at target $\left(\Delta Y_{B}\right)$ & $<2.0$ & $\mathrm{~mm}$ \\
Horn Peak Current, LE10 config. $\left(I_{H}\right)$ & $-185<I_{H}<-175$ & $\mathrm{kA}$ \\
Target $Z$ position, LE10 config. (Tarz) & $3850<$ Tar $z<4000$ & mills $[104]$ \\
\hline
\end{tabular}

The rate of NuMI neutrino candidates satisfying the simple VHits $[0]<6$ and THits $[0]>200$ selection cuts is measured to be:

$$
0.52 \pm 0.01 \nu / \text { P.O.T. }
$$

(NuMI $\nu$ Candidates per proton delivered to the NuMI Target)

Figure G.3 shows the stability of this rate as a function of time starting on May 20, 2005. The lines in the figure are a fit to a horizontal line and a line with a slope, both of which are consistent with a constant rate with very good goodness of fit. 


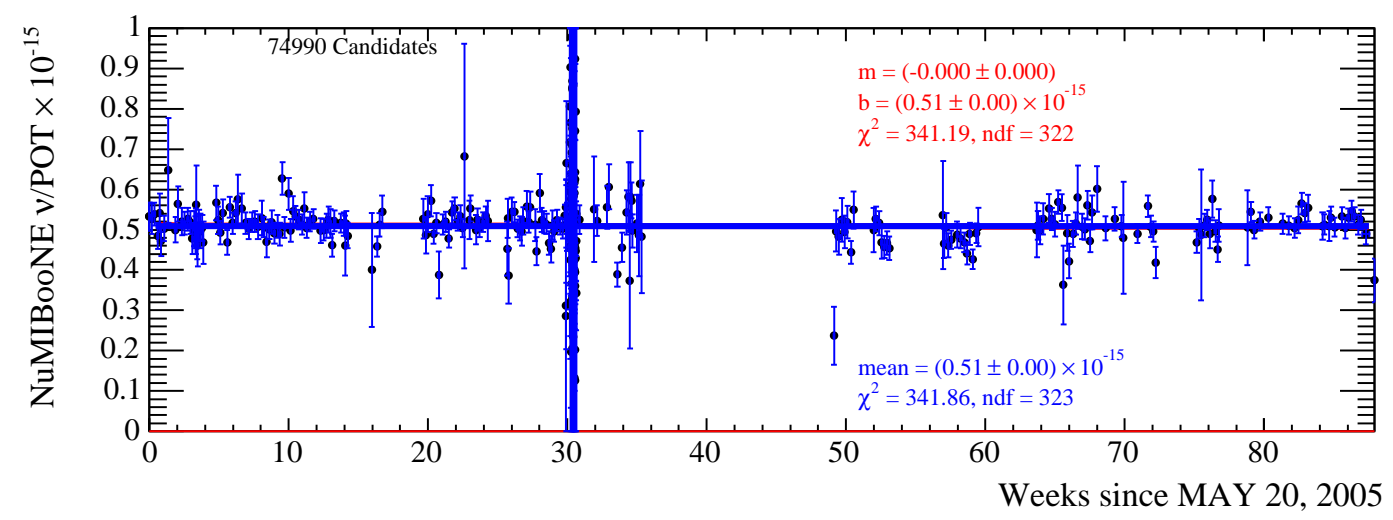

Figure G.3: Rate of NuMI neutrino candidates as a function of time. The uncertainties shown are statistical and include a $2 \%$ error in POT counting and calibration [103].

\section{Observing the NuMI beamline geometry with exiting events}

A sample of events with the highest energies is useful to try to observe the geometry of the NuMI beamline. It is expected that high energy neutrinos will produce tracks that in average point back to their parent meson decay position, and will punch through the detector veto on their way out while firing a cluster of veto PMT's that can be lined up with the reconstructed track direction. Using the cuts of Table G.3 a sample with these characteristics was accumulated.

Since most of the neutrinos seen by the detector at its off-axis position come from meson decays in the vicinity of the NuMI target, the coarse selection of exiting events in Table G.3 will predominantly come from that area, and in average their reconstructed tracks will point back to the NuMI target within a few meters. This is clearly seen in Figure G.4(d), which shows the distribution of the angle between the projection of the target production line and the MiniBooNE $\mathrm{Z}$ axis on the X-Z plane. The schematic drawing in Figure G.4(e) shows the angles between the beamlines in the plain and elevation views (X-Z and Y-Z projections) as determined from survey measurements of various beamline elements (See Appendix H). The angle that the 
Table G.3: Selection cuts for a sample of exiting NuMI events with a cluster of veto PMT's along the end of the reconstructed track and high energy deposition. Distributions for these events are shown in Figure G.4.

Selection cuts, NuMI exiting sample

\begin{tabular}{lc}
\hline \hline Loose beam window cut & $1 \mu \mathrm{s}<$ Avg. Time $<12 \mu \mathrm{s}$ \\
Punch-through Veto once & $6<$ VHits $<30$ \\
Single cluster present in veto & $\mathrm{NC}=1$ \\
Small angle between track and vertex-cluster line & $\cos \theta_{\text {cl }}^{\text {veto }}<0.95$ \\
High energy deposition & $E_{\text {vis }}>1.5 \mathrm{GeV}$ \\
\hline
\end{tabular}

target production line makes with the MiniBooNE beamline in the elevation view projection can be obtained from the distribution for $\cos \theta_{Y}$ in Figure G.4(c). Both angles as determined from the sample of exiting events are in excellent agreement with those derived from survey measurements, and are summarized in Table G.4.

Table G.4: Comparison of measurements of the vertical and horizontal inclinations of the target production line (as defined in Appendix $\mathrm{H}$ ). $\theta_{X Z}$ is the angle between the $\mathrm{XZ}$ projection of this line and the MiniBooNE $\mathrm{Z}$ axis. $\theta_{Y}$ is the angle of this line wrt. the vertical. Measurements are from Figure G.4.

\begin{tabular}{c|cc}
\hline Angle & From Survey Measurements & Angular distributions of exiting events \\
\hline$\theta_{Y}$ & $24.88^{\circ}$ & $(24.93 \pm 0.44)^{\circ}$ \\
$\theta_{X Z}$ & $87.50^{\circ}$ & $(87.25 \pm 0.41)^{\circ}$ \\
\hline
\end{tabular}




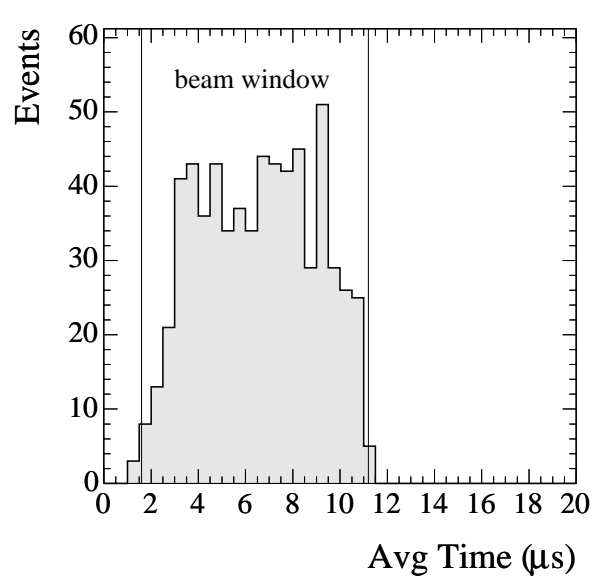

(a) Event time distribution

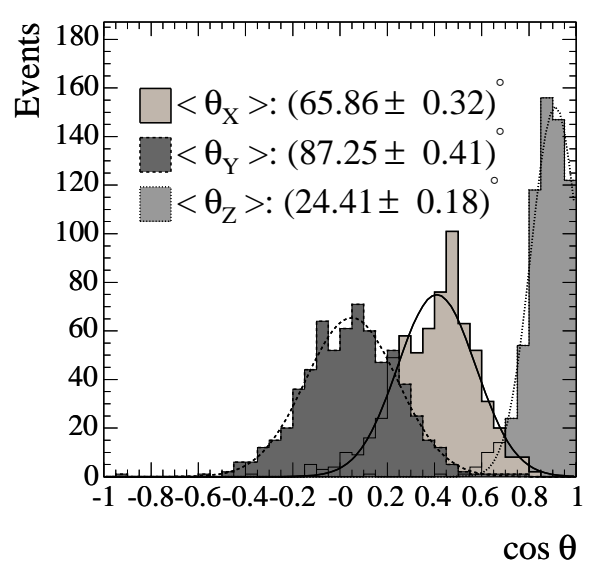

(c) Director cosines of reconstructed

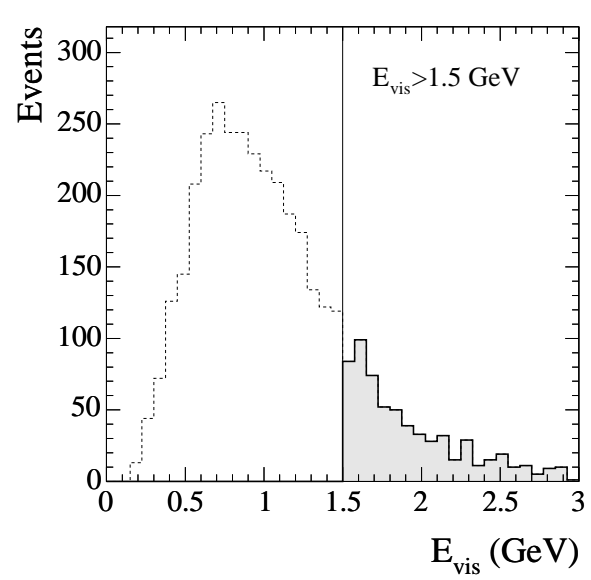

(b) Visible energy

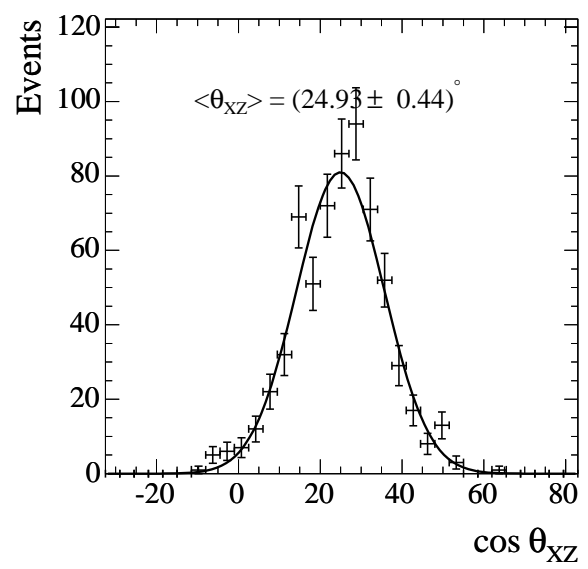

(d) Angle of X-Z projection of track.

tracks. The curves are Gaussian fits.
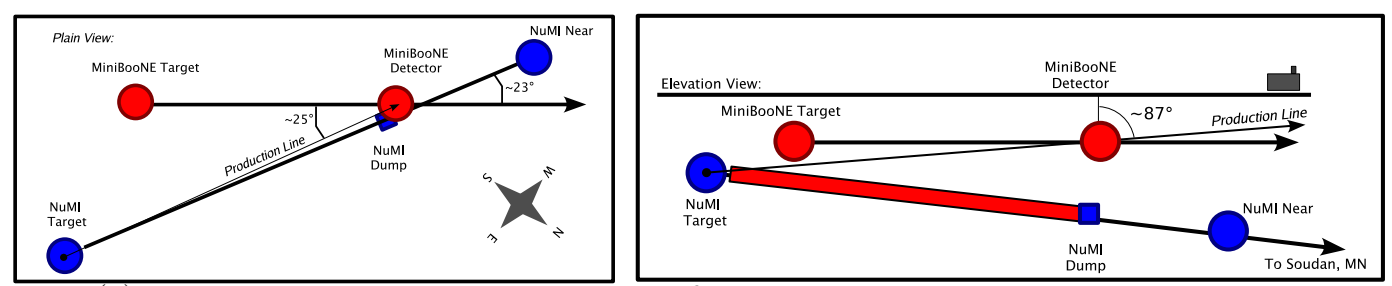

(e) Plain and elevation cartoon views of the NuMI and MiniBooNE beamlines.

Figure G.4: Distributions for events exiting the tank (See Table G.3). In average the tracks point in the direction of the target production line, defined in Appendix $\mathrm{H}$. The $\sim 25^{\circ}$ angle between the plane view projections of the MiniBooNE $Z$ axis and the target production line is visible in $(\mathrm{d})$. The $\sim 3^{\circ}$ upward inclination of the target production line is visible in the $\cos \theta_{\mathrm{Y}}$ distribution in $(\mathrm{c})$. 


\section{G.1.1 NuMI flux predictions and Monte Carlo simulation}

\section{Flux prediction}

A FLUKA[147] based simulation of the NuMI target produces a table of particles escaping the target surface which is passed to a GEANT3 based simulation of the beamline geometry (gnumi-v19 [176]) that includes the propagation in the magnetic field of the two-horn system and subsequent decay of mesons and muons in the decay pipe. Since neutrinos of lower energies than those typically observed in the MINOS detectors are be visible to MiniBooNE, a reduction of the kinematic threshold to track particles in the simulation was implemented.

In the FLUKA portion of the simulation this modification consisted in reducing the threshold on hadrons produced at the target from the standard $0.5 \mathrm{GeV}$ in MINOS, to $10 \mathrm{MeV}$, leaving the threshold for neutrons at $19.6 \mathrm{MeV}$ as indicated in the FLUKA manual pages ${ }^{3}$. The threshold reduction in the gnumi-v19 part of the simulation is implemented in the input card file definition and is set to cut hadrons with momenta lower than $1 \mathrm{eV}$.

MINOS collaborators ${ }^{4}$ produced a special beam simulation with the above modifications. The produced gnumi-v19 ntuples were translated into the format that is output by the MiniBooNE beam Monte Carlo programs BooNEG4Beam and BooBeamNTN.

The gnumi-v19 ntuples contain the momentum of the decayed meson in the laboratory frame and the energy of its daughter neutrino in the meson rest frame. A random neutrino direction is chosen by boosting into the parent meson rest frame and randomly throwing a neutrino vector uniformly in a spherical casquet large enough to

3 The modification occurs in the parameter PART-THR in the input definitions file target_2_.inp, F.Yumiceva private comm.

${ }^{4}$ S. Kopp and Z. Pavlovich, University of Texas, Austin. 


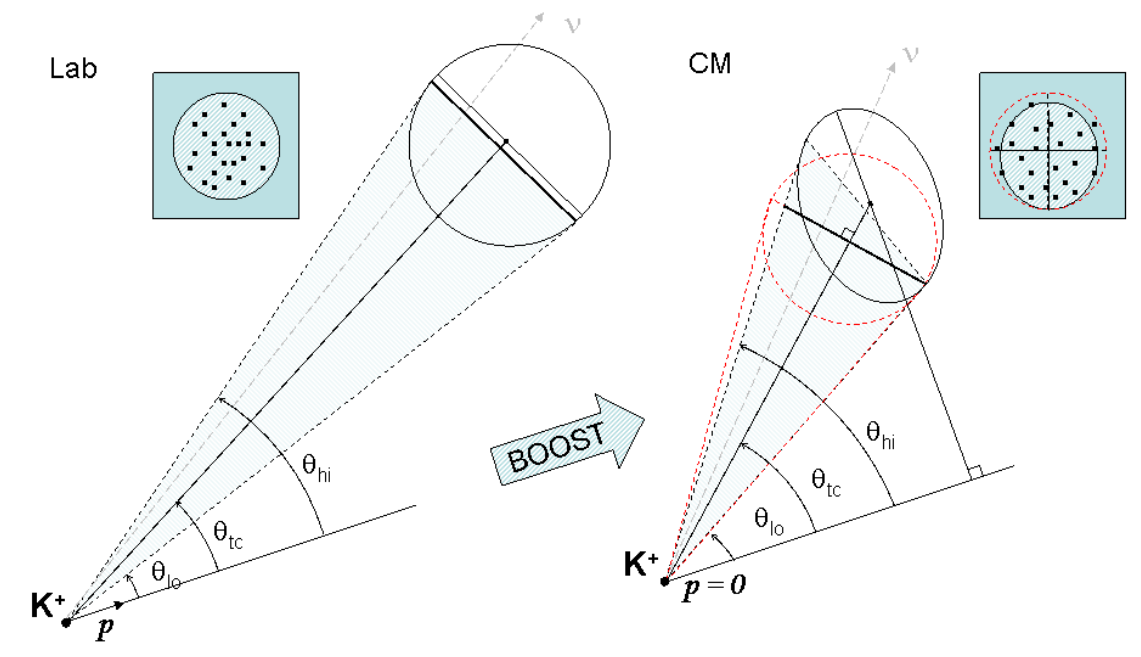

Figure G.5: Illustration of the random throw of neutrino vectors. For a given parent (here a $K^{+}$) and its decay neutrino: first boost into the meson rest frame and throw vectors uniformly over a spherical casquet shown as the dashed circle in the square on the right. Only vectors that hit the detector area are kept and boosted back to the laboratory frame, where the throws are no longer uniform.

enclose the Lorentz-contracted detector ellipse in this frame. As illustrated in Figure G.5 a vector is kept only if it points to the inside of the detector and is used to form the neutrino momentum vector for that particular decay. The neutrino directions are isotropic in the parent rest frame but will not be so in the laboratory frame. The algorithm described above takes care of this without needing to transform the detector solid angle between the center of mass and laboratory frame. Details of the processing chain to generate neutrino event rates using the standard MiniBooNE software tools can be found elsewhere [175].

The predicted neutrino flux at the MiniBooNE detector from the decay of mesons produced in the NuMI target, beamline, and dump is shown in Figure G.6, where the contribution from $\nu_{\mu}, \nu_{e}, \bar{\nu}_{\mu}$ and $\bar{\nu}_{e}$ are separately displayed. The prominent peak at $\sim 2 \mathrm{GeV}$ is dominated by the off-axis decays of $K^{+}$into $\nu_{\mu}$, while the peak around 
$\sim 200 \mathrm{MeV}$ is dominated by the decays of $\pi^{+}$into $\nu_{\mu}$, as can be seen in Figure G.7(a). The location of both peaks corresponds to what is expected for a detection location of $110 \mathrm{mrad}$ away from the beam axis [177]. The composition of the neutrino flux as predicted by the simulation is shown in Table G.5.

Mesons from the target moving along the decay pipe decay into neutrinos with an energy spectrum determined by the off-axis angle of the detector. For kaons moving parallel to the beamline axis, there is a tight correlation between the energy $E_{\nu}$ and angle $\theta$ of the neutrino produced in the two-body decay $K^{+} \rightarrow \mu^{+} \nu_{\mu}$ :

$$
E_{\nu}=\frac{0.96 E_{K}}{1+\gamma^{2} \theta^{2}}
$$

where $\gamma=E_{K} / m_{K}$ is the Lorentz boost factor, and $E_{k}$ and $m_{k}$ are the energy and mass of the parent kaon respectively. For two-body pion decays $\pi^{+} \rightarrow \mu^{+} \nu_{\mu}$, one replaces the 0.96 by 0.43 . For non-zero $\theta$, and above a moderate energy threshold, the values of $E_{K}$ map into a narrow $E_{\nu}$ interval. In this way, positive kaons (pions) of various energies being focused by the NuMI horns contribute to the neutrino flux with energies around $\sim 2 \mathrm{GeV}(\sim 200 \mathrm{MeV})$. Note that the enhancing effect is absent for $\bar{\nu}_{\mu}$ from $\pi^{-}$and $K^{-}$decays, whose contribution to the flux is shown in Figure G.7(c).

More details on the simulation of NuMI events and a thorough study of the $\nu_{\mu}$ CCQE and $\nu_{e}$ CCQE samples can be found in Ref.[178]. 
Table G.5: Predicted contributions to the neutrino fluxes from the NuMI beam in Figure G.7 per secondary particle produced at the target. Each neutrino type comes from a chain that ends with the indicated process.

\begin{tabular}{c|lr}
\hline \hline Neutrino flavor & \multicolumn{1}{c}{ Process } & Contribution \\
\hline$\nu_{\mu}(66.7 \%)$ & $\mathrm{pC} \rightarrow(\ldots) \rightarrow \pi^{+} \rightarrow \nu_{\mu}$ & $84.7 \%$ \\
& $\mathrm{pC} \rightarrow(\ldots) \rightarrow K^{+} \rightarrow \nu_{\mu}$ & $14.8 \%$ \\
\hline & $\mathrm{pC} \rightarrow(\ldots) \rightarrow K_{L}^{0} \rightarrow \nu_{\mu}$ & $<0.5 \%$ \\
\hline$\nu_{e}(2.0 \%)$ & $\mathrm{pC} \rightarrow(\ldots) \rightarrow K^{+} \rightarrow \nu_{e} \rightarrow \nu_{e}$ & $12.5 \%$ \\
& $\mathrm{pC} \rightarrow(\ldots) \rightarrow K_{L}^{0} \rightarrow \nu_{e}$ & $38.3 \%$ \\
$\bar{\nu}_{\mu}(30.7 \%)$ & $\mathrm{pC} \rightarrow(\ldots) \rightarrow \pi^{-} \rightarrow \bar{\nu}_{\mu}$ & $49.2 \%$ \\
\hline & $\mathrm{pC} \rightarrow(\ldots) \rightarrow K_{L}^{0} \rightarrow \bar{\nu}_{\mu}$ & $7.8 \%$ \\
\hline $\bar{\nu}_{e}(0.7 \%)$ & $\mathrm{pC} \rightarrow(\ldots) \rightarrow K^{-} \rightarrow \bar{\nu}_{e}$ & $0.9 \%$ \\
\hline \hline
\end{tabular}




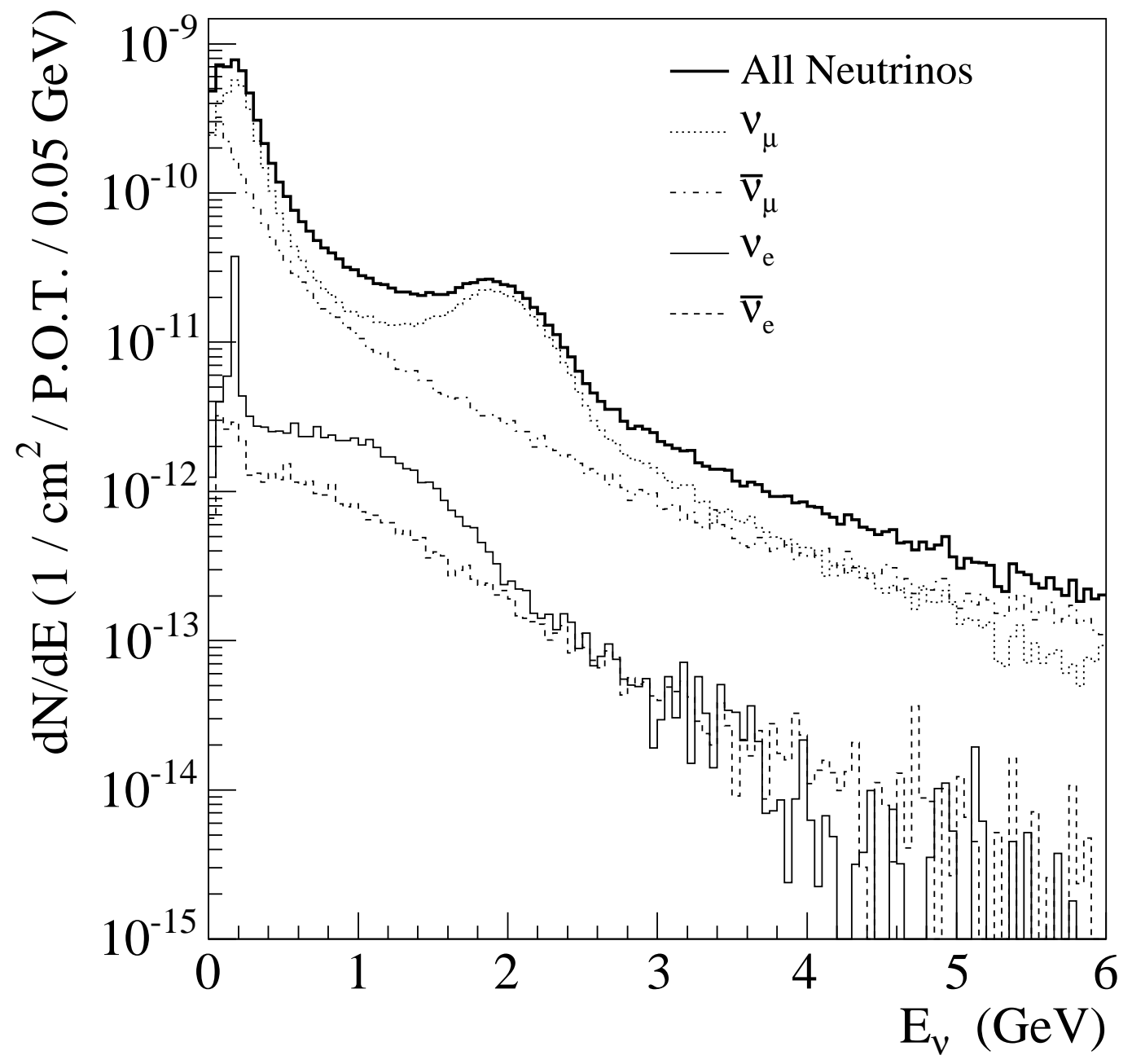

Figure G.6: Total predicted neutrino flux from the NuMI beamline at the MiniBooNE detector. 


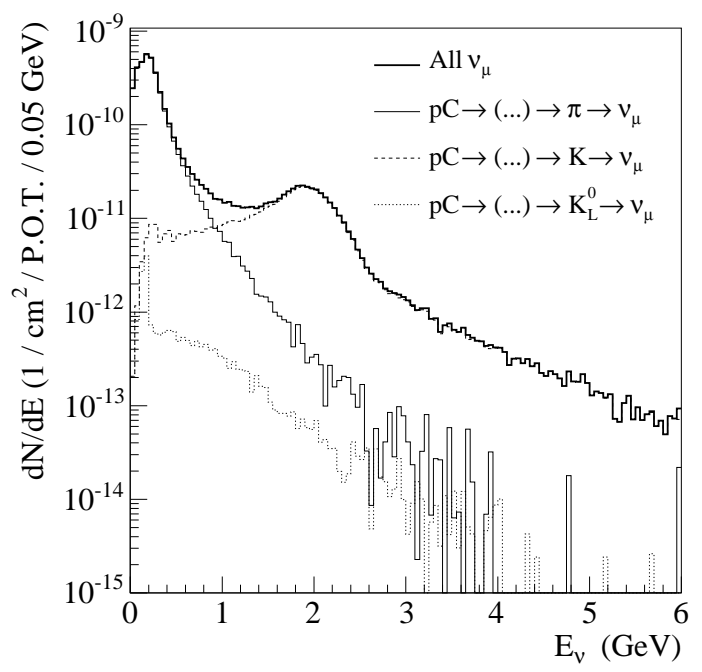

(a) $\nu_{\mu}$ flux.

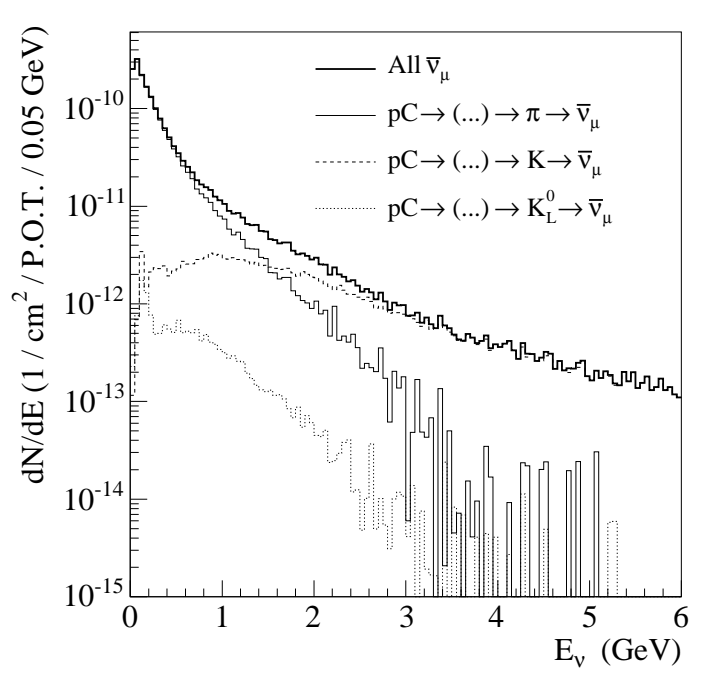

(c) $\bar{\nu}_{\mu}$ flux.

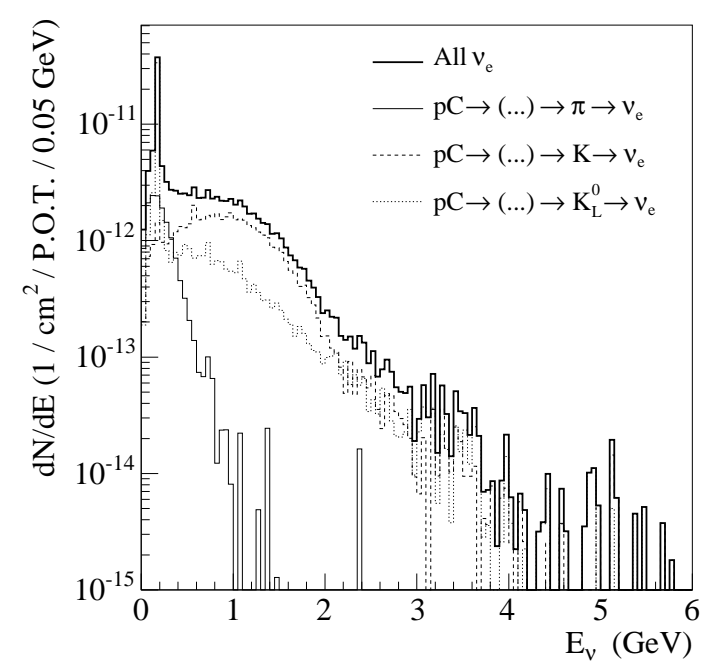

(b) $\nu_{e}$ flux.

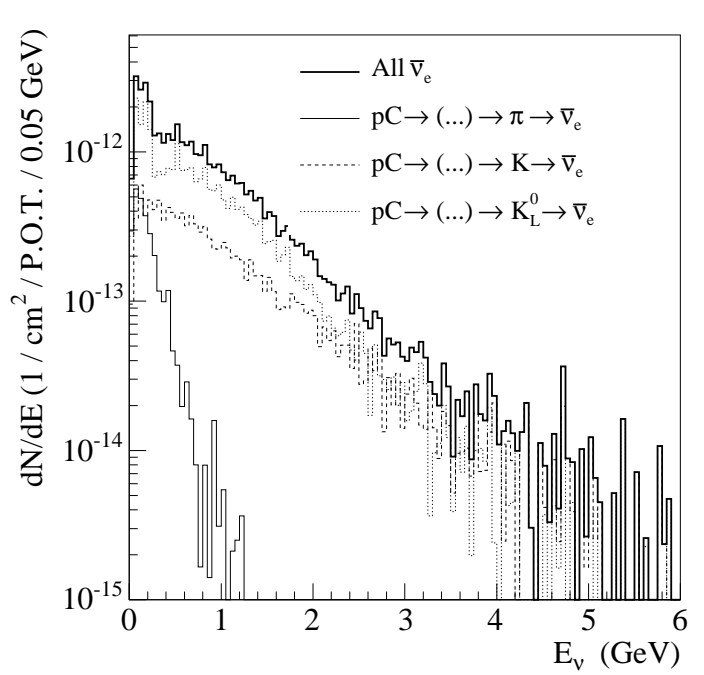

(d) $\bar{\nu}_{e}$ flux.

Figure G.7: Predicted flux from the NuMI beam in the MiniBooNE detector as a function of neutrino energy for each neutrino type. The components from different secondary particles produced at the target are shown. The percent contribution of these various sources is shown in table G.5. 


\section{Appendix $\mathrm{H}$}

\section{Geometry of the NuMI and}

\section{MiniBooNE Beamlines}

This section contains calculations of geometrical relations between the NuMI and MiniBooNE beamline based on the Fermilab's "SITE" coordinates of the elements considered. Position vectors and rotation matrices are calculated as well.

\section{H.1 Positions of elements in site coordinates}

The positions of the relevant objects are given in Table H.1 ${ }^{1}$. The origin of the NuMI coordinate system is defined as the insertion point of the first horn (MCZERO), and the $\mathrm{Z}$ axis of the NuMI coordinate frame points towards a point in the NuMI beam DUMP (also denominated DENCU by MINOS people). The NuMI Y axis runs perpendicular to the $\mathrm{Z}$ axis and towards the sky. The $\mathrm{X}$ axis is such that the coordinate frame is right handed.

\footnotetext{
${ }^{1}$ From survey data: Virgil B. (09/26/2005), and Ray Stefanski (MiniBooNE).
} 
Table H.1: Site Coordinates in feet $(1 \mathrm{ft}=0.3048$ meters $)$

\begin{tabular}{|l|c|ccc|}
\hline Element & Shorthand & X(easting) & Y(northing) & Z(elevation) \\
\hline NuMI Near Detector & NEAR & 97305.82 & 99374.69 & 416.31 \\
MiniBooNE Detector & MB & 98097.99 & 98860.11 & 729.22 \\
MiniBooNE Target & MBTGT & 99222.64 & 97485.55 & 723.00 \\
NuMI Target & NUMITGT & 100308.90 & 97790.20 & 614.15 \\
NuMi Beam Stop & DUMP & 98221.11 & 98891.75 & 476.25 \\
\hline
\end{tabular}

The MiniBooNE beamline coordinate system has its origin inside the MiniBooNE horn at a point on the target axis. The $\mathrm{Z}$ axis runs towards the MiniBooNE detector, and the $\mathrm{Y}$ axis is pointed upwards. The $\mathrm{X}$ axis is such that the coordinate system is right handed.

\section{H.2 Definition of vectors}

We first define a set of vectors between the different points in Table H.1 and use them to define the NuMI and MiniBooNE beamlines, as well as some auxiliary lines.

Table H.2: Definition of vectors. Long and Perp refer to the longitudinal and perpendicular components along the NuMI beam line.

\begin{tabular}{|cccccc|}
\hline Vector & Origin & End Point & length $(\mathrm{ft})$ & long $(\mathrm{ft})$ & perp $(\mathrm{ft})$ \\
\hline Q & NUMITGT & MB & 2458.87 & 2443.80 & 271.80 \\
V & NUMITGT & DUMP & 2364.59 & 2364.59 & 0.0 \\
P & DUMP & MB & 283.11 & 79.21 & 271.80 \\
R & MBTGT & MB & 1776.03 & 1632.97 & 698.34 \\
\hline
\end{tabular}

We define the target production line as the vector from NuMI target to MB detector 
(vector $\mathbf{Q}$ in Table H.2). A large fraction of the neutrinos reaching the tank will be produced in the vicinity of the NuMI target, making this line a good approximation to the neutrino direction. Vector $\mathbf{V}$ in Table H.2 defines the NuMI beamline. The NuMI Beamline is inclined downwards to point to the far detector at Soudan MN with a tilt angle relative to the horizontal of $-0.058 \mathrm{rad}$, as calculated from table H.2 Another production line can be considered for neutrinos produced at the NuMI beam dump. This line is defined by vector $\mathbf{P}$ in Table H.2. The MiniBooNE detector is at a distance of $283.11 \mathrm{ft}$ from the NuMI beam dump. Looking along the NuMI beamline up to the location of the MB detector, it subtends an angle of:

\section{Off-axis angle of MB wrt. NuMI beamline $=0.1108 \mathrm{rad}$}

We calculate the angle between the two beamlines using the dot and cross products of vectors $\mathbf{R}$ and $\mathbf{V}$. The absolute angle between the beamlines (NuMI Z and $\mathrm{MB} \mathrm{Z}$ in SITE coordinate system) is:

\section{Absolute angle between beamlines: $\alpha=23.15^{\circ}$}

\section{H.3 Aereal view projections of the beamlines}

In a plain or aereal view of the Fermilab site, the NuMI and MiniBooNE beamlines intersect at a point slightly north-east of the MiniBoNE detector. Table H.3 shows the easting and northing dimensions of the two beamlines as defined earlier, and their length in the aereal projections.

Taking the dot product of the aereal view projections of $\mathbf{R}$ and $\mathbf{V}$ yields the value $R p \cdot V p=5555379.92 \mathrm{ft}^{2}$, from which the angle between the beamlines in the aereal view is extracted using the norm of both vectors:

Angle between beamlines in aereal view projection: $\alpha=22.89^{\circ}$ 
Table H.3: Dimensions in site coordinates $\mathrm{X}$ (easting), $\mathrm{Y}$ (northing) of the aereal view projections of the vectors defining the NuMI and MB beamlines. Lengths are in feet.

\begin{tabular}{cccc}
\hline Vector & $\Delta X(\mathrm{ft})$ & $\Delta Y(\mathrm{ft})$ & length of projection $(\mathrm{ft})$ \\
$\mathbf{R}$ & -1124.65 & 1374.56 & 1776.02 \\
$\mathbf{V}$ & -3003.08 & 1584.48 & 3395.45 \\
\hline
\end{tabular}

\section{H.4 MiniBooNE position in NuMI coordinates}

Using dot and cross products of vectors $\mathrm{R}$ and $\mathrm{V}$ we calculate the MB position in the NuMI coordinate system.

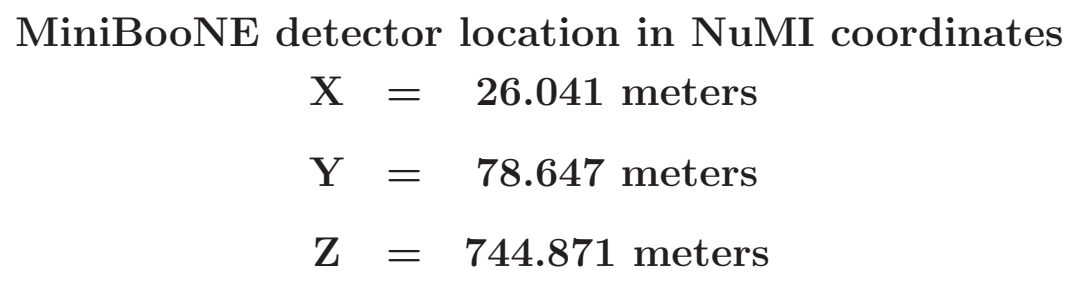

Check: From the calculated coordinates get the distance from the NuMI origin to MB:

$$
\begin{aligned}
\text { Distance NUMITGT to MB } & =2458.87 \mathrm{ft} \\
\text { Perpendicular distance } & =271.80 \mathrm{ft}
\end{aligned}
$$

which match the norm of the vector $\mathbf{Q}$ and its perpendicular component calculated in Table H.2.

Define the vector RMBTGTtoNEAR from MB target to NuMI near det:

Distance MBTGT to NEAR $=825.615$ meters

\section{H.5 Basis transformation Matrices}

The following matrices have as columns the components of the basis unit vectors of the NuMI and MB frames respectively, expressed in SITE coordinates. 
Matrix to transform from SITE coordinates to NuMI coordinates:

$\operatorname{TMnumi}(i, j)=$

$$
\begin{array}{ccc}
-0.466645367 & -0.0515797363 & -0.882939201 \\
-0.884444516 & 0.0272141944 & 0.465851141 \\
-1.13942832 \mathrm{E}-18 & 0.998298011 & -0.0583187926
\end{array}
$$

Matrix to transform from SITE coordinates to MiniBooNE coordinates:

$\operatorname{TMmb}(\mathrm{i}, \mathrm{j})=$

$$
\begin{array}{ccc}
-0.773955535 & 0.0022177138 & -0.633236063 \\
-0.633239946 & -0.00271052368 & 0.773950789 \\
1.54245475 \mathrm{E}-19 & 0.999993867 & 0.00350216977
\end{array}
$$

To convert a vector from SITE coordinates to either NuMI or MB coordinates multiply the transpose of the previous matrices times the vector in SITE coordinates.

\section{H.5.1 Examples}

Using matrix TMnumi: $(\text { TMnumi })^{T} \times Q \quad \mathrm{X}=26.041$ meters

MB Det coordinates in NuMI frame:

$\mathrm{Y}=78.647$ meters

$\mathrm{Z}=744.871$ meters

Let $\mathrm{N}$ be the vector from NUMITGT to MBTGT.

Using matrix TMnumi: $(\text { TMnumi })^{T} \times N$

$$
\begin{gathered}
\mathrm{X}=236.631 \text { meters } \\
\mathrm{Y}=47.672 \text { meters } \\
\mathrm{Z}=247.140 \text { meters }
\end{gathered}
$$




\section{H.6 Production line vectors in MB coordinates}

First we calculate in MB coordinates the components of the unit vector pointing from NUMITGT to MB:

Using the matrix TMmb: $(\mathrm{TMmb})^{T} \times Q$

Coordinates of prod. line vector in MB frame (unit norm)

$$
\mathrm{X}=0.42037005, \mathrm{Y}=0.0436241162, \mathrm{Z}=0.906303458,
$$

with direction defined by the polar angles:

$$
\theta=87.50^{\circ}, \phi=24.88^{\circ}
$$

Now we calculate in MB coordinates the components of the unit vector pointing from DUMP to MB:

Using matrix TMmb: $(\mathrm{TMmb})^{T} \times P$

Coordinates of DUMP prod line in MB frame (unit norm)

$$
\mathrm{X}=0.407340799, \mathrm{Y}=0.892864152, \mathrm{Z}=0.192008017,
$$

with direction defined by the polar angles:

$$
\theta=26.76^{\circ}, \phi=64.76^{\circ}
$$

\section{H.7 Rotation matrices in polar angle format}

Polar angle format of rotation matrices is used for example in GEANT3 to rotate elements with respect to their mother volume. The rotation matrices defined here rotate from SITE coordinates to either NuMI or MB frames. They are constructed by giving the polar coordinates of the 3 axes of the corresponding frame with respect to the SITE coordinate system. 
Table H.4: Polar angles of NuMI and MiniBooNE coordinate axes in SITE coordinates.

\begin{tabular}{l|cc} 
Axis & $\theta$ & $\phi$ \\
\hline \hline NuMI X & 89.99999750 & 242.18323884 \\
NuMI Y & 3.34331757 & 152.18324135 \\
NuMI Z & 93.34331506 & 152.18324135 \\
\hline MiniBooNE X & 89.99999750 & 219.28956537 \\
MiniBooNE Y & 0.20065995 & 309.28956787 \\
MiniBooNE Z & 89.79933754 & 129.28956787 \\
\hline
\end{tabular}

The parameters of a GEANT3 rotation matrix are given as a vector of 6 entries: $\left(\theta_{x}, \phi_{x}, \theta_{y}, \phi_{y}, \theta_{z}, \phi_{z}\right)$

\section{H.8 Transformation matrix from NuMI to MB:}

We calculate the transformation matrix from NuMI to MB using the matrices TMnumi and TMmb that transform SITE coordinates to either coordinate system. The matrix TMnumi_to_mb is simply:

$$
\text { TMnumi_to_mb }=(\mathrm{TMmb})^{T} \times \text { TMnumi }
$$

Note that as opposed to TMnumi and TMmb, TMnumi_to_mb has the unit vectors of the MB frame expressed in NuMI coordinates as rows, not columns. Hence to transform a vector from NuMI coordinates to MB coordinates we do not take the transpose:

If Xnumi(i) is expressed in NuMI coordinates, then Xmb(i) obtained by: 


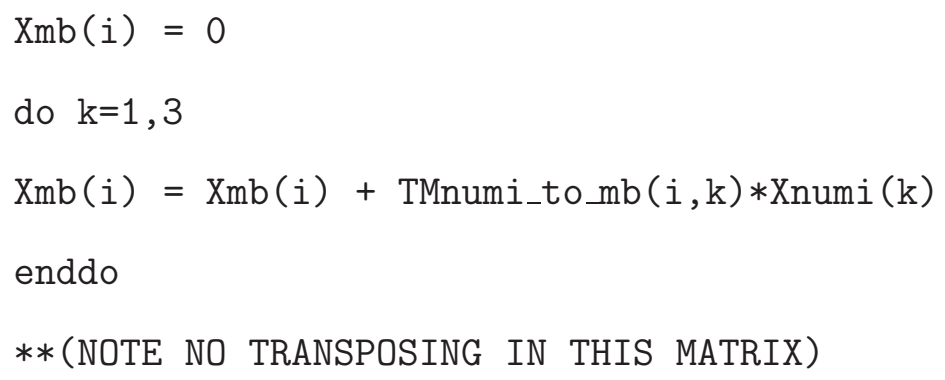

is the same vector expressed in MB coordinates. The matrix TMnumi is given by:

TMnumi_to_mb $(\mathrm{i}, \mathrm{j})=$

$$
\begin{array}{ccc}
0.921228362 & 0.0226873074 & 0.38836013 \\
0.00136242193 & 0.998103735 & -0.061539242 \\
-0.389019856 & 0.0572208054 & 0.919450559
\end{array}
$$

\section{H.8.1 MiniBooNE frame axes in NuMI coordinates}

Finally, we calculate the polar angle format of the rotation matrix from the NuMI frame to the MiniBooNE frame.

Table H.5: Polar angles of MiniBooNE coordinate axes in NuMI coordinates.

\begin{tabular}{ccc} 
Axis & $\theta$ & $\phi$ \\
\hline \hline MB X in NuMI & 67.14749780 & 1.41075143 \\
MB Y in NuMI & 93.52816554 & 89.92178821 \\
MB Z in NuMI & 23.15411018 & 171.63238370
\end{tabular}

As before, these define the parameters for a GEANT3 rotation. 


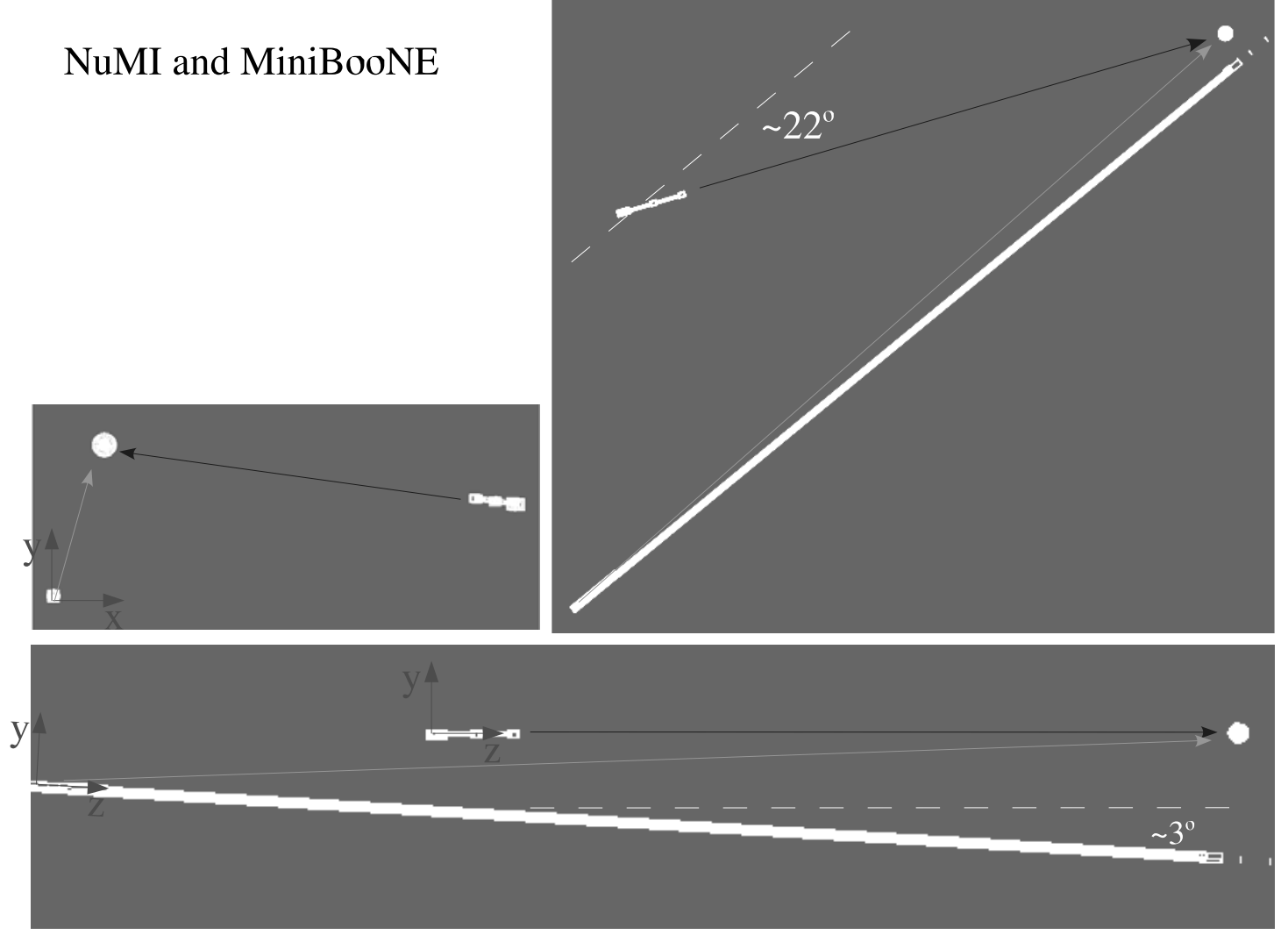

Figure H.1: GEANT4 rendering of the NuMI and MiniBooNE beamlines. To make this drawing the geometry files from the two simulations were combined in a single one. To position and rotate the MiniBooNE beamline relatively to the NuMI beamline, the matrices and vectors described in this note were used. Shown are parallel projections onto the NuMI $\mathrm{XY}, \mathrm{XZ}$, and YZ planes. The dark arrow represents the trajectory of a neutrino from the MiniBooNE beamline to the detector. The light gray line represents the trajectory of a typical neutrino from the vicinity of the NuMI target to the MiniBooNE detector. The axes and labels were added by hand. 


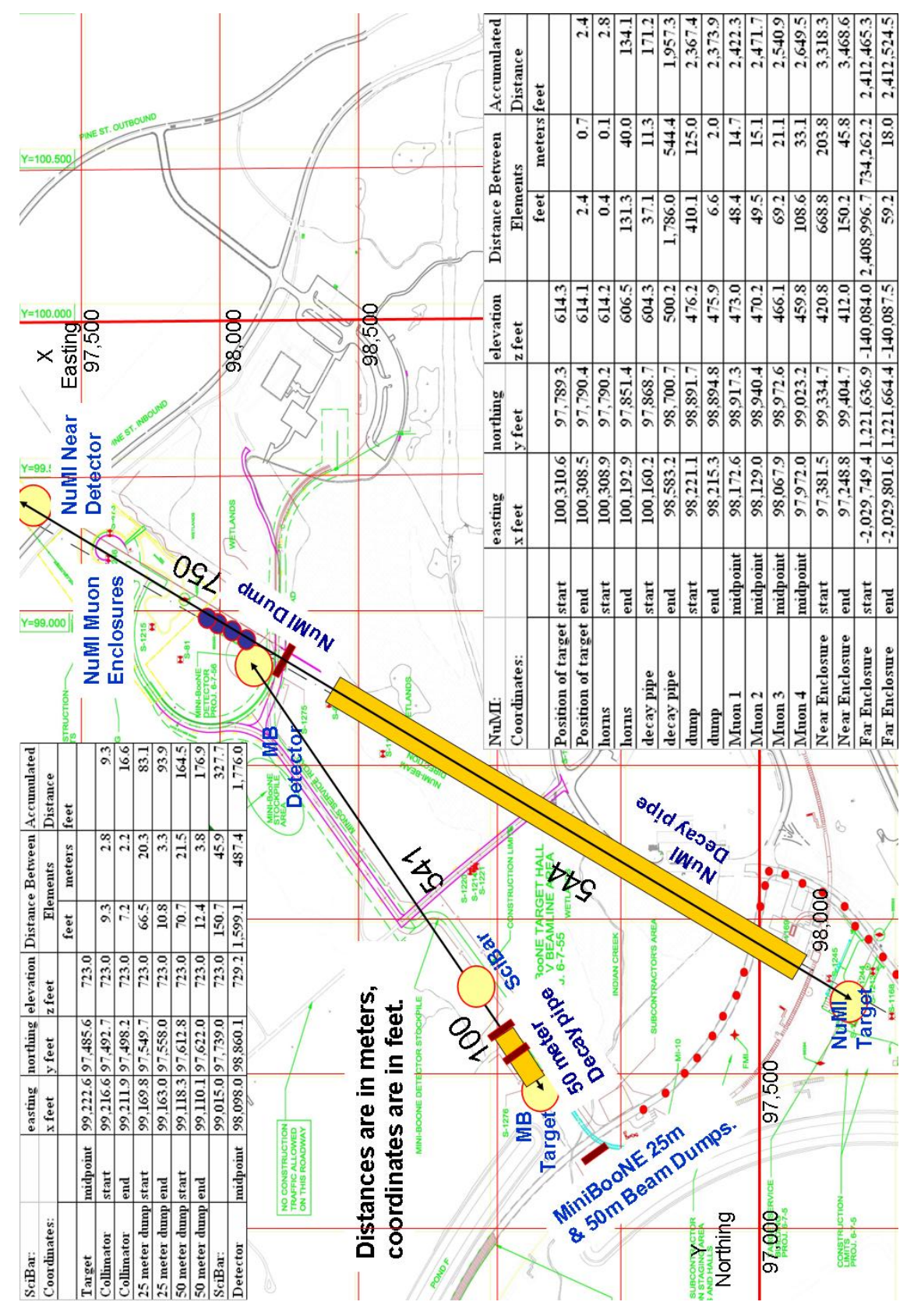

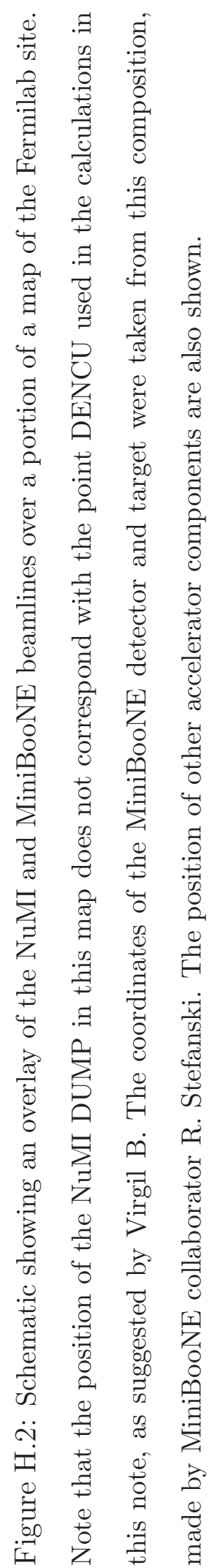

